\title{
High-sensitivity cardiac troponins in health and disease
}

Citation for published version (APA):

Klinkenberg, L. J. J. (2016). High-sensitivity cardiac troponins in health and disease. [Doctoral Thesis, Maastricht University]. Maastricht University. https://doi.org/10.26481/dis.20160304lk

Document status and date:

Published: 01/01/2016

DOI:

10.26481/dis.20160304lk

Document Version:

Publisher's PDF, also known as Version of record

\section{Please check the document version of this publication:}

- A submitted manuscript is the version of the article upon submission and before peer-review. There can be important differences between the submitted version and the official published version of record.

People interested in the research are advised to contact the author for the final version of the publication, or visit the DOI to the publisher's website.

- The final author version and the galley proof are versions of the publication after peer review.

- The final published version features the final layout of the paper including the volume, issue and page numbers.

Link to publication

\footnotetext{
General rights rights.

- You may freely distribute the URL identifying the publication in the public portal. please follow below link for the End User Agreement:

www.umlib.nl/taverne-license

Take down policy

If you believe that this document breaches copyright please contact us at:

repository@maastrichtuniversity.nl

providing details and we will investigate your claim.
}

Copyright and moral rights for the publications made accessible in the public portal are retained by the authors and/or other copyright owners and it is a condition of accessing publications that users recognise and abide by the legal requirements associated with these

- Users may download and print one copy of any publication from the public portal for the purpose of private study or research.

- You may not further distribute the material or use it for any profit-making activity or commercial gain

If the publication is distributed under the terms of Article $25 \mathrm{fa}$ of the Dutch Copyright Act, indicated by the "Taverne" license above, 
High-sensitivity cardiac troponins in health and disease 
High-sensitivity cardiac troponins in health and disease Lieke J.J. Klinkenberg

ISBN 9789090295732

Cover illustration by Ramon Kool

Layout by Lieke J.J. Klinkenberg

Printed by Impreso

(C) Copyright Lieke J.J. Klinkenberg, Maastricht 2016 


\title{
High-sensitivity cardiac troponins in health and disease
}

\author{
Proefschrift
}

ter verkrijging van de graad van doctor aan de Universiteit Maastricht, op gezag van de Rector Magnificus, Prof. dr. L.L.G. Soete, volgens het besluit van het College van Decanen, in het openbaar te verdedigen

op vrijdag 4 maart 2016 om 14:00 uur

door

Lieke Jeanne Josseline Klinkenberg 


\section{Promotores}

Prof. dr. M.P. van Dieijen-Visser

Prof. dr. L.J.C. van Loon

\section{Copromotor}

Dr. S.J.R. Meex

Beoordelingscommissie

Prof. dr. C.D.A. Stehouwer (voorzitter)

Prof. dr. F.S. Apple (University of Minnesota, Minneapolis, USA)

Prof. dr. J.F.C. Glatz

Prof. dr. W.W. van Solinge (Universitair Medisch Centrum Utrecht)

Prof. dr. L.J. de Windt

The research described in this thesis was supported by a grant of Stichting De Weijerhorst and Stichting Sint Annadal.

Financial support by Roche Diagnostics for the publication of this thesis is gratefully acknowledged. Abbott Diagnostics Division and Beckman Coulter are also gratefully acknowledged for their financial support. 


\section{Contents}

$\begin{array}{lll}\text { Chapter } 1 \text { General introduction } & 7\end{array}$

Chapter $2 \quad$ Circulating cardiac troponin T exhibits a diurnal rhythm 21

Chapter 3 A diurnal rhythm of cardiac troponin: consequences for the 41 diagnosis of acute myocardial infarction

Chapter 4 Prognostic value of basal high-sensitive cardiac troponin levels 69 on mortality in the general population: a systematic review and meta-analysis

Chapter 5 Strong link between basal and exercise-induced cardiac troponin T levels: do both reflect risk?

Chapter 6 Effect of antioxidant supplementation on exercise-induced cardiac troponin release in cyclists: a randomized trial

Chapter 7 Cardiac troponin T and I release following a $30 \mathrm{~km}$ run: comparison of troponin kinetics and effect of remote ischemic preconditioning

Chapter 8 General discussion

Summary

Samenvatting

Valorization

Dankwoord

Curriculum vitae

List of publications 


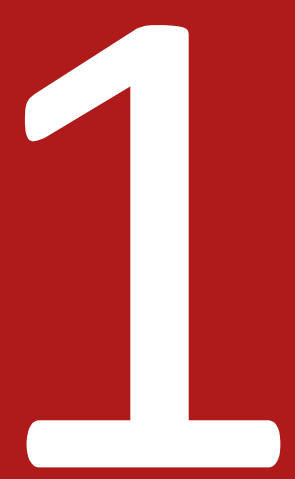


General introduction 


\section{Cardiac troponins and myocardial infarction}

\section{Acute myocardial infarction}

Acute myocardial infarction (AMI) is characterized by cardiomyocyte death due to prolonged ischemia. ${ }^{1}$ The initial step in the development of AMI is an imbalance between the oxygen demand of the myocardium and the oxygen supply by the coronary arteries, which is generally the result of a coronary occlusion. ${ }^{2,3}$ Early identification of patients is of critical importance to initiate medical treatment and management. A clinical assessment of myocardial ischemia, consisting of an electrocardiogram and detailed evaluation of chest discomfort, is indispensable in the diagnostic workup of AMI. While these clinical tools have insufficient accuracy of their own, the biochemical hallmark of the diagnostic workup of AMI is the measurement of cardiac troponins (cTn) in blood. ${ }^{1-7}$

A

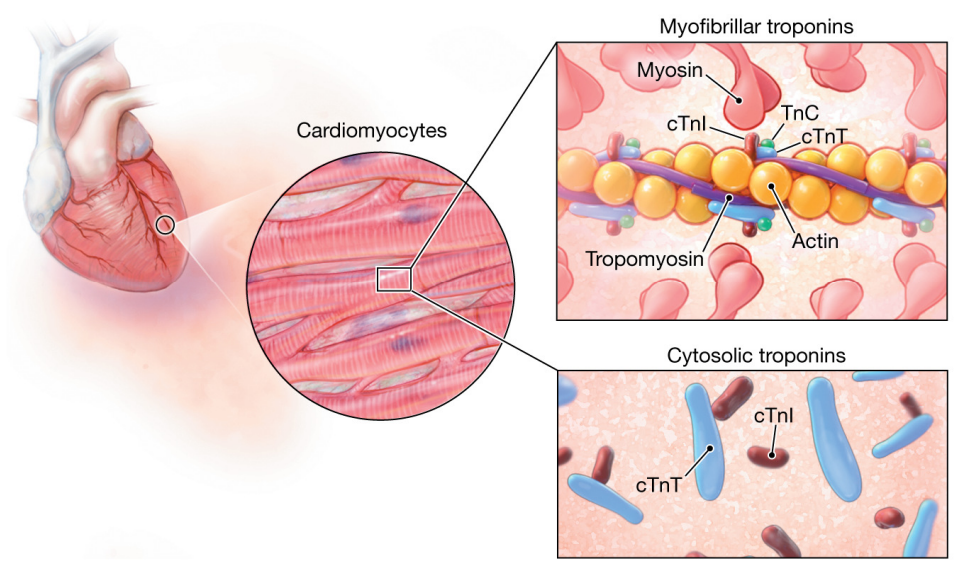

B

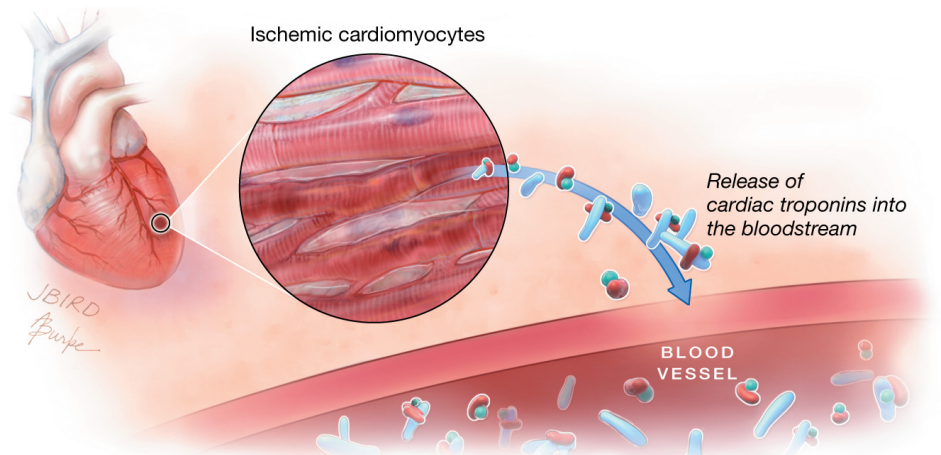

Figure 1. Release of cardiac troponins following ischemic cardiac injury.

A. Physiology of the healthy myocardium. The cTn complex, consisting of troponin C (TnC), cardiac troponin T (cTnT) and I (cTnl) is structurally bound to the actin filament. A small proportion of cTn possibly exists as unbound protein in the cytosol. B. Following myocardial damage, cTn are released into the bloodstream. Used with permission of the American Medical Association. ${ }^{8}$ 


\section{(Patho-)physiology of cardiac troponins}

cTn are components of the thin filament of the contractile apparatus of cardiomyocytes and fulfill a critical role in the regulation of excitation-contraction coupling in the heart (figure 1). The troponin complex is composed of three protein subunits; cardiac troponin I (cTnI), cardiac troponin T (cTnT) and troponin C (TnC). cTn are compartmented in the cardiomyocyte as structurally bound to actin filaments, with possibly a small proportion (3 - 8\%) existing as unbound protein in the cytosol. ${ }^{9-11}$ Whereas cardiac and skeletal muscle share the TnC isoform, cTnl and cTnT have unique cardiac isoforms. ${ }^{12}$ This makes both cTnl and cTnT suitable for diagnostic use in cardiac pathology.

Figure 2. Release curve of cardiac troponins after the onset of symptoms of myocardial ischemia. In general, cTnl and cTnT display a monophasic and biphasic release curve, respectively. This figure was used with permission of Creative Commons. $^{14}$

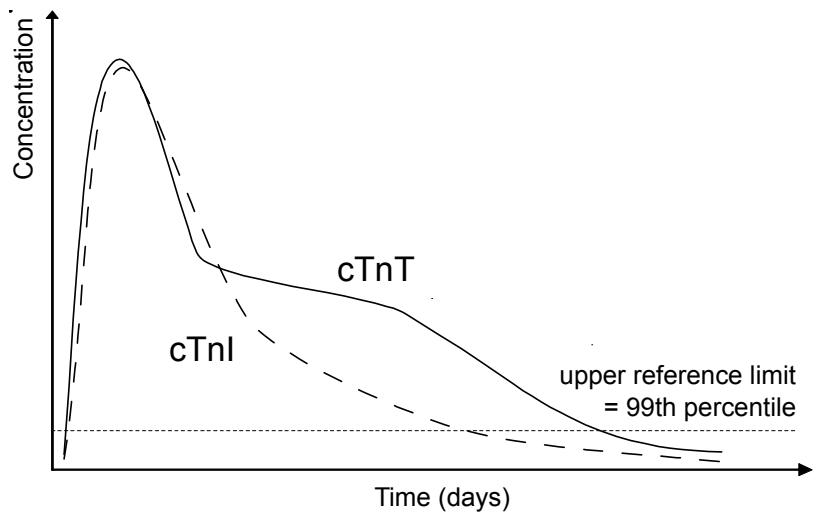

Following an AMI, there is a distinct release kinetics of cTn into the circulation (figure 2). The cTnT release curve is typical biphasic; with an early steep rise in concentrations, followed by a high peak and gradual decrease over several days after the onset of myocardial ischemia. ${ }^{13,14}$ It has been hypothesized that the initial increase of cTnT results from the release of the cytosolic pool. Subsequently, the second prolonged elevation has been suggested to result from the breakdown of the contractile apparatus. ${ }^{9,10,15}$ In contrast to $\mathrm{CTnT}$, cTnl concentrations generally decrease more rapidly after reaching peak concentrations, resulting in a monophasic pattern. The exact reason for this different release kinetics is unknown, although cTnT differs from cTnl with respect to a higher molecular weight and higher fraction of unbound $\mathrm{CTnT}^{16}$ Hence, in conjunction with typical clinical symptoms of myocardial ischemia, detection of elevated circulating $\mathrm{cTnl}$ or $\mathrm{CTnT}$ above the $99^{\text {th }}$ percentile of a healthy reference population confirm a diagnosis of AMI.

High-sensitivity cardiac troponin assays

Recent advances in assay technology have led to an enhancement in the analytical performance of the troponin assays and therefore an improved ability to detect very 
low circulating levels of cTn with a higher precision. Therefore, these so-called (high-) sensitivity assays are therefore able to detect cTn in healthy individuals from the general population, enabling a precise determination of the $99^{\text {th }}$ percentile cut-off value for the diagnosis of AMI. ${ }^{17}$ CTnT assays are only produced by a single manufacturer (Roche Diagnostics), due to intellectual property restriction. This is in contrast to cTnl, where more than twenty assays are nowadays commercially available with different analytical characteristics. ${ }^{18,19}$ The analytical characteristics of the troponin assays used throughout this thesis are summarized in table 1.

Table 1. Analytical characteristics of the troponin assays used throughout this thesis.

\begin{tabular}{llllll}
\hline Troponin & Manufacturer & $\begin{array}{l}\mathrm{LoB}^{\mathrm{a}}, \\
\mathrm{ng} / \mathrm{L}\end{array}$ & $\begin{array}{l}\mathrm{LoD}{ }^{\mathrm{b}}, \\
\mathrm{ng} / \mathrm{L}\end{array}$ & $\begin{array}{l}99^{\text {th }} \text { percentile, } \\
\mathrm{ng} / \mathrm{L}\end{array}$ & $\begin{array}{l}10 \% \mathrm{CV}, \\
\mathrm{ng} / \mathrm{L}\end{array}$ \\
\hline hs-cTnT & Roche Diagnostics & 3.0 & 5.0 & 14.0 & 13.0 \\
hs-cTnl & Abbott Diagnostics & $0.7-1.3$ & $1.1-1.9$ & 26.2 & 4.7 \\
s-cTnl & Beckman Coulter & $<10.0$ & 10.0 & 40.0 & 40.0 \\
\hline
\end{tabular}

${ }^{a}$ The highest concentration that can be observed (with a 95\% probability) for a sample that does not contain the analyte of interest. ${ }^{b}$ The lowest concentration of an analyte that can be detected (with a $95 \%$ probability). LoB; limit of blank, LoD; limit of detection, CV; coefficient of variation, s-cTnl; sensitive cardiac troponin I.

To classify troponin assays according to their analytical performance, Apple et. al. proposed a two criteria 'scorecard'. ${ }^{20}$ Based on the recommendation of the current universal definition of $\mathrm{AMI}$ to measure the $99^{\text {th }}$ percentile value with an optimal imprecision, assays are designated as 'not acceptable' (coefficient of variation [CV] $>20 \%$ ), 'clinically usable' (CV >10 - $\leq 20 \%$ ) or 'guideline acceptable' (CV $\leq 10 \%)$. In addition, four assay levels are defined according to the percentage of healthy reference individuals who have a measurable cTn value: 'level 1' (contemporary, <50\%), 'level 2' (first generation high-sensitivity [hs], 50 - 75\%), 'level 3' (second generation hs, 75 - 95\%), and 'level 4' (third generation hs, >95\%). For example, both the hs-cTnT assay of Roche Diagnostics and hs-cTnl assay of Abbott Diagnostics are designated as 'level 4 guideline acceptable assays'.17,20

Diagnosis of myocardial infarction by high-sensitivity troponin assays

Several large clinical trials have demonstrated that the advent of the high-sensitivity troponin assays has resulted in an increase in the diagnostic accuracy at the time of patient presentation to the emergency department. ${ }^{21,22}$ This superior performance of hs-cTnl and hs-cTnT in comparison to the contemporary assays is the result of an improved diagnostic sensitivity. These assays allow therefore a rapid rule-in of $\mathrm{AMI}$ and reduce morbidity and mortality through early initiation of evidence-based treatment and management. ${ }^{23}$ 
However, the improved sensitivity for AMI has come at a cost of specificity. Although elevated circulating hs-cTn indicate myocardial injury, cTn are not exclusively released as a result of ischemic cardiomyocyte death. ${ }^{13}$ Any acute or chronic condition that injures cardiomyocytes may lead to measurable and/or elevations of circulating hscTn. ${ }^{8,13,24}$ Acute conditions that for example can elevate hs-cTn include acute decompensated heart failure, pulmonary embolism, sepsis, endocarditis and stroke. ${ }^{25-29}$ In addition, persistent low-level elevations in hs-cTn are frequently measured among patients with chronic conditions such as chronic heart failure, stable coronary artery disease, type 2 diabetes mellitus and chronic kidney disease. ${ }^{30-33}$ While hs-cTnl and hsCTnT are not specific for any particular mechanism of myocardial injury, solitary elevations of hs-cTn with levels exceeding the $99^{\text {th }}$ percentile limit are not distinctive between cTn release resulting from an acute or chronic event. Therefore, the current universal definition of AMl is based on the detection of a rise and/or fall of cTn (cTnl or CTnT) above the $99^{\text {th }}$ percentile of a healthy reference population together with evidence of ischemia either by clinical symptoms, electrocardiographic findings or imaging techniques. ${ }^{1}$

\section{Serial testing of cardiac troponins}

The critical tool in the diagnostic workup of AMI is serial testing and the assessment of kinetic changes in hs-cTn. The detection of a rise and/or fall of hs-cTn in the definition of $\mathrm{AMI}$ is based on the assumption that concentrations of hs-cTn tend not to change acutely in individuals with chronic conditions. ${ }^{1}$ The universal definition of AMI however does not provide a substantiated guidance regarding the magnitude of the change required to discriminate between AMI-induced cTn release and other causes of circulating $c T n .{ }^{1}$ In the past few years, several biological variation studies have been published addressing this unmet clinical need. These studies are generally based upon healthy subjects and collected series of blood samples at standardized time intervals while minimizing pre-analytical variation. ${ }^{34}$ Subsequently, aliquots are stored appropriately and hs-cTn are measured in duplicate fashion in a single analytical run. The variance constituents of this dataset can be calculated accordingly and are composed of analytical variation $\left(\mathrm{CV}_{\mathrm{A}}\right)$, biological variation or within-person variation $\left(\mathrm{CV}_{\mathrm{i}}\right)$ and betweenperson variation $\left(\mathrm{CV}_{\mathrm{G}}\right)$ (figure 3 ). This design enables to derive so-called reference change values (RCV), according to the formula: $R C V=V 2 \cdot Z \cdot V\left(C V_{A}^{2}+C V_{i}^{2}\right)$, where $Z$ is a constant, depending on the statistical probability (usually a Z-score of 1.96 for $95 \%$ probability). ${ }^{35}$ The statistically calculated threshold-value distinguishes 'true changes' from 'noise' or random physiological fluctuations. All conducted studies so far have measured the biological variation of hs-cTn over a short time window ( 4 - 6h) and have 
been unable to verify the general, critical pre-condition of using RCV; that serial values of hs-cTn show true random variation without correlation between successive results. $^{34,36-39}$

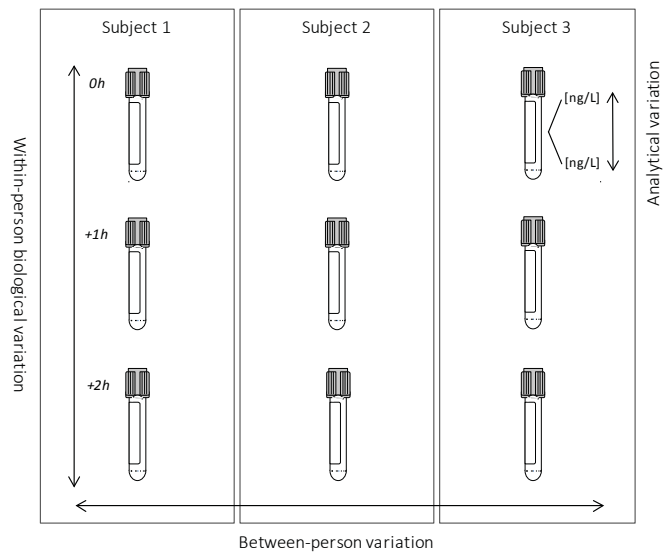

Figure 3. Design of biological variation studies. Biological variation studies are based on the collection of series of blood samples from several individuals to calculate betweenperson variation, within-person biological variation and analytical variation.

\section{Prognostic value of cardiac troponins}

It is inevitably clear that elevated levels of hs-cTn are associated with an increased risk of developing cardiovascular events and all-cause or cardiovascular mortality, regardless of an acute or chronic setting of myocardial injury, such as acute chest pain, acute dyspnea, type 2 diabetes, stable coronary artery disease, and chronic kidney disease. ${ }^{32,33,40-42}$ In addition, the detection of hs-cTn in almost every individual of the general population has expanded the possible use of cTn from diagnostic care to risk stratification and prognostic medicine. It has become clear that even in the general population and when adjusted for traditional cardiovascular risk factors, hs-cTn are important predictors for an adverse outcome. ${ }^{43-45}$ Nevertheless, this prognostic value of hs-cTn in the general population has not been systematically assessed.

\section{Exercise-induced cardiac troponins release}

\section{Elevation of cardiac troponins with endurance-type exercise}

Prolonged endurance-type exercise by recreational athletes, such as (half-)marathon running and cycling, has been associated with an acute impairment in cardiac function and release of cTn into the circulation. ${ }^{46-50}$ The kinetics of exercise-induced cTn release consists of an initial peak during exercise, followed by a second peak 3 - 6h upon cessa- 
tion of exercise. ${ }^{51}$ Within 24 - 48h, circulating cTn decrease significantly and generally normalize to baseline concentrations. ${ }^{49,51-55}$ Post-exercise concentrations of hs-cTn can vary tremendously among athletes, ranging from concentrations below the diagnostic cut-off for AMI to more than ten times the $99^{\text {th }}$ percentile limit..$^{50}$ In an attempt to understand the heterogeneity of exercise-induced cTn release, several studies have examined possible predisposing factors, such as exercise modalities or athletes' characteristics. These studies have reported that the cTn response to exercise is strongly related to the intensity and the duration of the exercise and inversely associated with the training experience of the athlete. ${ }^{56-61}$

\section{Potential mechanisms for exercise-induced troponin release}

Since it is well-known that regular exercise training reduces cardiovascular disease risk, ${ }^{62,63}$ it seems counter-intuitive to define circulating cTn as a result of irreversible cardiomyocyte injury. Nevertheless, there are no conclusive data supporting a pathological or physiological nature of cTn release in response to exercise. ${ }^{46}$ There are two prevailing hypotheses: the physiological concept is based on the assumption that exerciseinduced cTn release reflects reversible cardiomyocyte injury, characterized by a change in cardiomyocyte permeability and subsequent release of the cytosolic cTn pool, followed by cardiomyoyte repair and resulting in myocardial hypertrophy. ${ }^{46}$ This is in contrast to the pathological model, which is based on the hypothesis that exercise-induced cTn release is the result of irreversible cardiomyocyte injury, followed by scarring and fibrotic replacement of the myocardium. ${ }^{46}$

\section{Strategies to study troponin release in response to exercise}

Since intensive endurance-type exercise involves the integration of muscular, cardiovascular and neurological processes that function cooperatively to transfer energy into velocity and power, ${ }^{64}$ it is challenging to investigate in vivo contributing mechanisms responsible for the release of cTn associated with endurance-type exercise. Several components of the altered physiological environment of the cardiomyocyte during exercise have been suggested, such as an imbalance in oxygen supply and demand, elevations in reactive oxygen species and increased mechanical stress. ${ }^{65,66}$

It has been demonstrated that exhaustive exercise results in the formation of reactive oxygen species in the myocardium. ${ }^{67,68}$ Furthermore, the significant association of oxidative stress markers with levels of cTn in these exercise animal models, has resulted in the hypothesis that oxidative stress contributes to cTn release in the setting of prolonged endurance-type exercise. ${ }^{69}$ Supporting endogenous antioxidant systems with additional doses of antioxidants could therefore provide an important strategy to examine the role of reactive oxygen species as an underlying biological mechanism for exercise-induced cTn release. 
The contribution of an imbalance in oxygen supply and demand to cTn release following prolonged exercise can be studied by remote ischemic preconditioning (RIPC). RIPC is a powerful non-invasive cardio-protective strategy, whereby brief episodes of non-lethal ischemia followed by reperfusion of one tissue or organ leads to the protec-

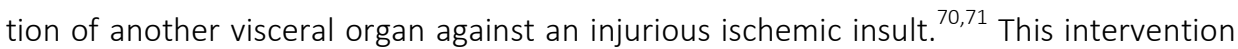
is safe, extremely simple and requires only a blood pressure cuff applied to the upper or lower limb. The RIPC stimulus is achieved by inflating the cuff to $>200 \mathrm{~mm} \mathrm{Hg}$ and then deflated after a predetermined period of time (typically $5 \mathrm{~min}$ ) for four cycles (figure 4). This strategy has been successfully translated to humans, and powerful cardiomyocyte protective effects have been reported in cardiac surgery and angioplasty, measured by post-surgery levels of hs-cTn and all-cause mortality. ${ }^{72-76}$ The exact nature of how challenging one vascular bed transduces to protection in another distant visceral organ is the subject of intense research. Two potential pathways have been postulated, a humoral pathway, involving a blood-borne mediator, and secondly a neuronal pathway. ${ }^{77,78}$ If an imbalance in oxygen supply and demand during prolonged endurancetype exercise contributes to cTn release, applying a RIPC stimulus would result in a decrease of post-exercise hs-cTn.

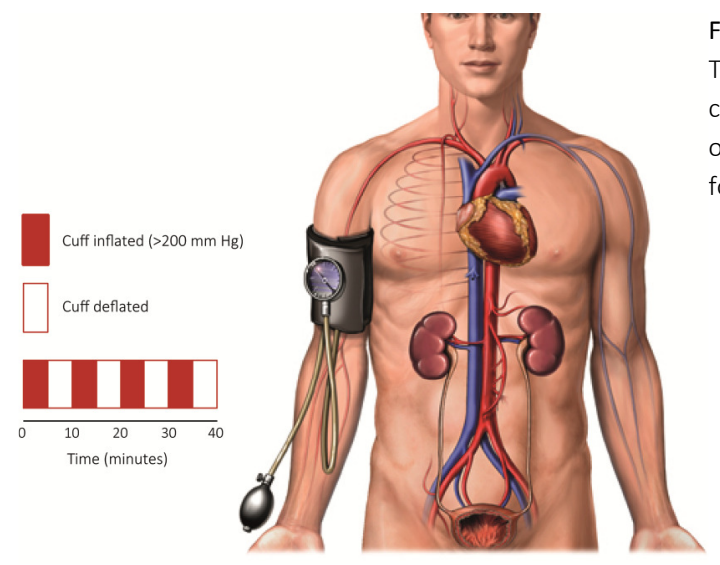

Figure 4. Remote ischemic preconditioning. The remote ischemic preconditioning stimulus consists of the inflation of a blood pressure cuff on an upper limb to induce transient ischemia, followed by cuff deflation.

\section{Outline of this thesis}

Cardiac troponins (cTn) are currently the most sensitive markers for the detection of myocardial injury and are therefore the biochemical gold standard to diagnose acute myocardial infarction (AMI). Major analytical refinements in the troponin assays have resulted in more sensitive and precise information regarding circulating levels of cardiac troponin I (cTnl) and T (cTnT). This resulted in the detection of previously unnoticed 
levels of cTn in a wide spectrum of acute and chronic diseases, that are inevitably associated with adverse outcomes. The critical tool in the diagnostic workup of AMI is therefore serial testing and the assessment of kinetic changes of hs-cTn. On the other hand, the accurate detection of cTn in almost every healthy individual of the general population has expanded the possible use of cTn from acute cardiac care to risk stratification. The single exception where it is unclear whether cTn are linked to adverse outcomes is the release of cTn during and after endurance-type exercise.

The aim of the first chapters of this thesis is to improve the interpretation of serial hs-cTn measurements. Chapter 2 challenges the critical assumption that hs-cTn fluctuate randomly in subjects with chronically elevated levels of hs-cTn. Specifically, this chapter shows that circulating hs-cTnT exhibits diurnal oscillation in male subjects with type 2 diabetes. Chapter 3 validates that the diurnal hs-cTnT rhythm is a general phenomenon, independent of sex and glucose metabolism status. The clinical impact of the diurnal rhythm on the diagnostic accuracy of hs-cTnT for AMI is additionally described in this chapter. Furthermore, this chapter evaluates whether (hs-)cTnl assays are equally affected by the clock time.

Chapter 4 describes a systematic assessment of the prognostic value of hs-cTn in the general population. By a meta-regression analysis, this chapter aims to compare the prognostic performance of hs-cTnl and hs-cTnT.

The objective of the last chapters of this thesis is to study exercise-induced cTn release. In order to understand the heterogeneous release of cTn following an acute bout of endurance-type exercise, a highly standardized assessment of the reproducibility and predisposing factors of cycling-induced hs-cTnT levels are described in Chapter 5. The effect of two interventions on cTn release following prolonged exercise is evaluated in the following two chapters. Chapter 6 describes the effect of antioxidant supplementation on exercise-induced hs-cTnT levels in cyclists. Chapter 7 investigates the effect of remote ischemic preconditioning on the release kinetics of hs-cTnl and hs-cTnT following a $30 \mathrm{~km}$ running trial. Chapter 8 contains a general discussion of the work presented in this thesis and directions for future research are provided. 


\section{References}

1. Thygesen K, Alpert JS, Jaffe AS, et al. Third universal definition of myocardial infarction. Eur Heart J. 2012;33(20):2551-2567.

2. Hamm CW, Bassand JP, Agewall S, et al. ESC Guidelines for the management of acute coronary syndromes in patients presenting without persistent ST-segment elevation: The Task Force for the management of acute coronary syndromes (ACS) in patients presenting without persistent ST-segment elevation of the European Society of Cardiology (ESC). Eur Heart J. 2011;32(23):2999-3054.

3. Amsterdam EA, Wenger NK, Brindis RG, et al. 2014 AHA/ACC Guideline for the Management of Patients With Non-ST-Elevation Acute Coronary Syndromes: A Report of the American College of Cardiology/American Heart Association Task Force on Practice Guidelines. J Am Coll Cardiol. 2014;64(24):e139-228.

4. Mueller C. Biomarkers and acute coronary syndromes: an update. Eur Heart J. 2014;35(9):552-556.

5. Thygesen K, Mair J, Giannitsis E, et al. How to use high-sensitivity cardiac troponins in acute cardiac care. Eur Heart J. 2012;33(18):2252-2257.

6. Thygesen K, Mair J, Katus $\mathrm{H}$, et al. Recommendations for the use of cardiac troponin measurement in acute cardiac care. Eur Heart J. 2010;31(18):2197-2204.

7. Morrow DA, Cannon CP, Jesse RL, et al. National Academy of Clinical Biochemistry Laboratory Medicine Practice Guidelines: Clinical characteristics and utilization of biochemical markers in acute coronary syndromes. Circulation. 2007;115(13):e356-375.

8. de Lemos JA. Increasingly sensitive assays for cardiac troponins: a review. JAMA. 2013;309(21):22622269.

9. Katus HA, Remppis A, Scheffold T, Diederich KW, Kuebler W. Intracellular compartmentation of cardiac troponin $T$ and its release kinetics in patients with reperfused and nonreperfused myocardial infarction. Am J Cardiol. 1991;67(16):1360-1367.

10. Remppis A, Scheffold T, Greten J, et al. Intracellular compartmentation of troponin T: release kinetics after global ischemia and calcium paradox in the isolated perfused rat heart. J Mol Cell Cardiol. 1995;27(2):793-803.

11. Voss EM, Sharkey SW, Gernert AE, et al. Human and canine cardiac troponin T and creatine kinase-MB distribution in normal and diseased myocardium. Infarct sizing using serum profiles. Arch Pathol Lab Med. 1995;119(9):799-806.

12. Parmacek MS, Solaro RJ. Biology of the troponin complex in cardiac myocytes. Prog Cardiovasc Dis. 2004;47(3):159-176.

13. Giannitsis E, Katus HA. Cardiac troponin level elevations not related to acute coronary syndromes. Nat Rev Cardiol. 2013;10(11):623-634.

14. Jacobs L, Mingels A, Van Dieijen-Visser M. Cardiac biomarkers in end-stage renal disease. In: Sahay M, ed. Chronic Kidney Disease and Renal Transplantation: InTech; 2011.

15. Antman EM. Decision making with cardiac troponin tests. N Engl J Med. 2002;346(26):2079-2082.

16. Agewall S, Giannitsis E, Jernberg T, Katus H. Troponin elevation in coronary vs. non-coronary disease. Eur Heart J. 2011;32(4):404-411.

17. Apple FS, Collinson PO. Analytical characteristics of high-sensitivity cardiac troponin assays. Clin Chem. 2012;58(1):54-61.

18. Cardinaels EP, Mingels AM, Jacobs LH, Meex SJ, Bekers O, van Dieijen-Visser MP. A comprehensive review of upper reference limits reported for (high-)sensitivity cardiac troponin assays: the challenges that lie ahead. Clin Chem Lab Med. 2012;50(5):791-806.

19. Panteghini M. Assay-related issues in the measurement of cardiac troponins. Clin Chim Acta. 2009;402(1-2):88-93.

20. Apple FS. A new season for cardiac troponin assays: it's time to keep a scorecard. Clin Chem. 2009;55(7):1303-1306.

21. Keller T, Zeller T, Ojeda F, et al. Serial changes in highly sensitive troponin assay and early diagnosis of myocardial infarction. JAMA. 2011;306(24):2684-2693.

22. Reichlin T, Hochholzer W, Bassetti S, et al. Early diagnosis of myocardial infarction with sensitive cardiac troponin assays. N Engl J Med. 2009;361(9):858-867. 
23. Mills NL, Churchhouse AM, Lee KK, et al. Implementation of a sensitive troponin I assay and risk of recurrent myocardial infarction and death in patients with suspected acute coronary syndrome. JAMA. 2011;305(12):1210-1216.

24. Kelley WE, Januzzi JL, Christenson RH. Increases of cardiac troponin in conditions other than acute coronary syndrome and heart failure. Clin Chem. 2009;55(12):2098-2112.

25. Peacock WFt, De Marco T, Fonarow GC, et al. Cardiac troponin and outcome in acute heart failure. $\mathrm{N}$ Engl J Med. 2008;358(20):2117-2126.

26. Muller-Bardorff M, Weidtmann B, Giannitsis E, Kurowski V, Katus HA. Release kinetics of cardiac troponin T in survivors of confirmed severe pulmonary embolism. Clin Chem. 2002;48(4):673-675.

27. Maeder M, Fehr T, Rickli H, Ammann P. Sepsis-associated myocardial dysfunction: diagnostic and prognostic impact of cardiac troponins and natriuretic peptides. Chest. 2006;129(5):1349-1366.

28. Purcell JB, Patel M, Khera A, et al. Relation of troponin elevation to outcome in patients with infective endocarditis. Am J Cardiol. 2008;101(10):1479-1481.

29. Sandhu R, Aronow WS, Rajdev A, et al. Relation of cardiac troponin I levels with in-hospital mortality in patients with ischemic stroke, intracerebral hemorrhage, and subarachnoid hemorrhage. Am J Cardiol. 2008;102(5):632-634.

30. Masson S, Anand I, Favero C, et al. Serial measurement of cardiac troponin T using a highly sensitive assay in patients with chronic heart failure: data from 2 large randomized clinical trials. Circulation. 2012;125(2):280-288.

31. Omland T, de Lemos JA, Sabatine MS, et al. A sensitive cardiac troponin T assay in stable coronary artery disease. N Engl J Med. 2009;361(26):2538-2547.

32. Everett BM, Cook NR, Magnone MC, et al. Sensitive cardiac troponin T assay and the risk of incident cardiovascular disease in women with and without diabetes mellitus: the Women's Health Study. Circulation. 2011;123(24):2811-2818.

33. deFilippi C, Seliger SL, Kelley W, et al. Interpreting cardiac troponin results from high-sensitivity assays in chronic kidney disease without acute coronary syndrome. Clin Chem. 2012;58(9):1342-1351.

34. Fraser CG. Biological variation: from principles to practice. Washington, DC: AACC Press; 2001.

35. Fraser CG, Harris EK. Generation and application of data on biological variation in clinical chemistry. Crit Rev Clin Lab Sci. 1989;27(5):409-437.

36. Wu AH, Lu QA, Todd J, Moecks J, Wians F. Short- and long-term biological variation in cardiac troponin I measured with a high-sensitivity assay: implications for clinical practice. Clin Chem. 2009;55(1):52-58.

37. Vasile VC, Saenger AK, Kroning JM, Klee GG, Jaffe AS. Biologic variation of a novel cardiac troponin I assay. Clin Chem. 2011;57(7):1080-1081.

38. Frankenstein L, Wu AH, Hallermayer K, Wians FH, Jr., Giannitsis E, Katus HA. Biological variation and reference change value of high-sensitivity troponin $\mathrm{T}$ in healthy individuals during short and intermediate follow-up periods. Clin Chem. 2011;57(7):1068-1071.

39. Vasile VC, Saenger AK, Kroning JM, Jaffe AS. Biological and analytical variability of a novel high-sensitivity cardiac troponin T assay. Clin Chem. 2010;56(7):1086-1090.

40. Haaf $P$, Reichlin $T$, Twerenbold $R$, et al. Risk stratification in patients with acute chest pain using three high-sensitivity cardiac troponin assays. Eur Heart J. 2014;35(6):365-375.

41. van Wijk S, Jacobs L, Eurlings LW, et al. Troponin T measurements by high-sensitivity vs conventional assays for risk stratification in acute dyspnea. Clin Chem. 2012;58(1):284-292.

42. Omland T, Pfeffer MA, Solomon SD, et al. Prognostic value of cardiac troponin I measured with a highly sensitive assay in patients with stable coronary artery disease. J Am Coll Cardiol. 2013;61(12):12401249.

43. Gore MO, Seliger SL, Defilippi CR, et al. Age- and sex-dependent upper reference limits for the highsensitivity cardiac troponin T assay. J Am Coll Cardiol. 2014;63(14):1441-1448.

44. Eggers KM, Lind L, Venge P, Lindahl B. Factors influencing the 99th percentile of cardiac troponin I evaluated in community-dwelling individuals at 70 and 75 years of age. Clin Chem. 2013;59(7):10681073.

45. Saunders JT, Nambi V, de Lemos JA, et al. Cardiac troponin T measured by a highly sensitive assay predicts coronary heart disease, heart failure, and mortality in the Atherosclerosis Risk in Communities Study. Circulation. 2011;123(13):1367-1376.

46. Whyte GP. Clinical significance of cardiac damage and changes in function after exercise. Med Sci Sports Exerc. 2008;40(8):1416-1423. 
47. George K, Spence A, Naylor LH, Whyte GP, Green DJ. Cardiac adaptation to acute and chronic participation in endurance sports. Heart. 2011;97(24):1999-2004.

48. Neumayr G, Pfister R, Mitterbauer G, Eibl G, Hoertnagl H. Effect of competitive marathon cycling on plasma $\mathrm{N}$-terminal pro-brain natriuretic peptide and cardiac troponin $\mathrm{T}$ in healthy recreational cyclists. Am J Cardiol. 2005;96(5):732-735.

49. Lippi G, Schena F, Dipalo M, et al. Troponin I measured with a high sensitivity immunoassay is significantly increased after a half marathon run. Scand J Clin Lab Invest. 2012;72(6):467-470.

50. Mingels A, Jacobs L, Michielsen E, Swaanenburg J, Wodzig W, van Dieijen-Visser M. Reference population and marathon runner sera assessed by highly sensitive cardiac troponin $\mathrm{T}$ and commercial cardiac troponin T and I assays. Clin Chem. 2009;55(1):101-108.

51. Middleton N, George K, Whyte G, Gaze D, Collinson P, Shave R. Cardiac troponin T release is stimulated by endurance exercise in healthy humans. J Am Coll Cardiol. 2008;52(22):1813-1814.

52. Scherr J, Braun S, Schuster T, et al. 72-h kinetics of high-sensitive troponin T and inflammatory markers after marathon. Med Sci Sports Exerc. 2011;43(10):1819-1827.

53. Tian Y, Nie J, Huang C, George KP. The kinetics of highly sensitive cardiac troponin T release after prolonged treadmill exercise in adolescent and adult athletes. J Appl Physiol (1985). 2012;113(3):418425.

54. Nie J, Tong TK, George K, Fu FH, Lin H, Shi Q. Resting and post-exercise serum biomarkers of cardiac and skeletal muscle damage in adolescent runners. Scand J Med Sci Sports. 2011;21(5):625-629.

55. Traiperm N, Gatterer H, Wille M, Burtscher M. Cardiac troponins in young marathon runners. Am J Cardiol. 2012;110(4):594-598.

56. Eijsvogels TM, Hoogerwerf MD, Maessen MF, et al. Predictors of cardiac troponin release after a marathon. J Sci Med Sport. 2015;18(1):88-92.

57. Mingels AM, Jacobs LH, Kleijnen VW, et al. Cardiac troponin T elevations, using highly sensitive assay, in recreational running depend on running distance. Clin Res Cardiol. 2010;99(6):385-391.

58. Serrano-Ostariz E, Terreros-Blanco JL, Legaz-Arrese A, et al. The impact of exercise duration and intensity on the release of cardiac biomarkers. Scand J Med Sci Sports. 2011;21(2):244-249.

59. Fu F, Nie J, Tong TK. Serum cardiac troponin T in adolescent runners: effects of exercise intensity and duration. Int J Sports Med. 2009;30(3):168-172.

60. Sahlen A, Gustafsson TP, Svensson JE, et al. Predisposing factors and consequences of elevated biomarker levels in long-distance runners aged $\geq 55$ years. Am J Cardiol. 2009;104(10):1434-1440.

61. Mehta R, Gaze D, Mohan S, et al. Post-exercise cardiac troponin release is related to exercise training history. Int J Sports Med. 2012;33(5):333-337.

62. Tanasescu M, Leitzmann MF, Rimm EB, Willett WC, Stampfer MJ, Hu FB. Exercise type and intensity in relation to coronary heart disease in men. JAMA. 2002;288(16):1994-2000.

63. Kokkinos P, Myers J, Faselis C, et al. Exercise capacity and mortality in older men: a 20-year follow-up study. Circulation. 2010;122(8):790-797.

64. Joyner MJ, Coyle EF. Endurance exercise performance: the physiology of champions. J Physiol. 2008;586(1):35-44.

65. Shave R, Baggish A, George K, et al. Exercise-induced cardiac troponin elevation: evidence, mechanisms, and implications. J Am Coll Cardiol. 2010;56(3):169-176.

66. Shave R, Oxborough D. Exercise-induced cardiac injury: evidence from novel imaging techniques and highly sensitive cardiac troponin assays. Prog Cardiovasc Dis. 2012;54(5):407-415.

67. Bejma J, Ramires P, Ji LL. Free radical generation and oxidative stress with ageing and exercise: differential effects in the myocardium and liver. Acta Physiol Scand. 2000;169(4):343-351.

68. Muthusamy VR, Kannan S, Sadhaasivam K, et al. Acute exercise stress activates Nrf2/ARE signaling and promotes antioxidant mechanisms in the myocardium. Free Radic Biol Med. 2012;52(2):366-376.

69. Nie J, Close G, George KP, Tong TK, Shi Q. Temporal association of elevations in serum cardiac troponin T and myocardial oxidative stress after prolonged exercise in rats. Eur J Appl Physiol. 2010;110(6):12991303.

70. Kharbanda RK, Nielsen TT, Redington AN. Translation of remote ischaemic preconditioning into clinical practice. Lancet. 2009;374(9700):1557-1565.

71. Shi W, Vinten-Johansen J. Endogenous cardioprotection by ischaemic postconditioning and remote conditioning. Cardiovasc Res. 2012;94(2):206-216. 
72. Thielmann $M$, Kottenberg $E$, Kleinbongard $P$, et al. Cardioprotective and prognostic effects of remote ischaemic preconditioning in patients undergoing coronary artery bypass surgery: a single-centre randomised, double-blind, controlled trial. Lancet. 2013;382(9892):597-604.

73. Sloth AD, Schmidt MR, Munk K, et al. Improved long-term clinical outcomes in patients with ST-elevation myocardial infarction undergoing remote ischaemic conditioning as an adjunct to primary percutaneous coronary intervention. Eur Heart J. 2014;35(3):168-175.

74. Hoole SP, Heck PM, Sharples L, et al. Cardiac Remote Ischemic Preconditioning in Coronary Stenting (CRISP Stent) Study: a prospective, randomized control trial. Circulation. 2009;119(6):820-827.

75. Hausenloy DJ, Mwamure PK, Venugopal V, et al. Effect of remote ischaemic preconditioning on myocardial injury in patients undergoing coronary artery bypass graft surgery: a randomised controlled trial. Lancet. 2007;370(9587):575-579.

76. Botker HE, Kharbanda R, Schmidt MR, et al. Remote ischaemic conditioning before hospital admission, as a complement to angioplasty, and effect on myocardial salvage in patients with acute myocardial infarction: a randomised trial. Lancet. 2010;375(9716):727-734.

77. Hausenloy DJ, Yellon DM. Remote ischaemic preconditioning: underlying mechanisms and clinical application. Cardiovasc Res. 2008;79(3):377-386.

78. Bell R, Yellon D. Surgery: Remote ischaemic conditioning-approaching prime time? Nat Rev Cardiol. 2013;10(11):619-621. 


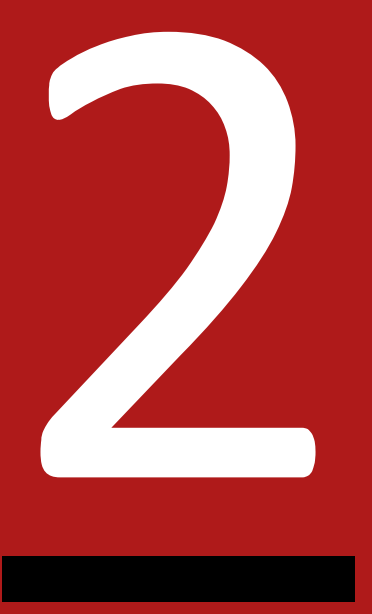




\section{Circulating cardiac troponin T exhibits a diurnal rhythm}

Lieke Klinkenberg*, Jan-Willem van Dijk*, Frans Tan, Luc van Loon, Marja van Dieijen-Visser, Steven Meex Journal of the American College of Cardiology. 2014. 63(17):1788-1795 


\section{Abstract}

Background The introduction of high-sensitivity cardiac troponin (hs-cTn) assays has improved sensitivity for acute myocardial infarction (AMI). However, many patients with a single positive hs-cTn test result do not have AMI. Therefore, the diagnosis of AMI relies strongly on serial testing and interpretation of hs-cTn kinetics. Essential in this regard is a profound understanding of the biological variation of hs-cTn. The aim of this study was to test the unverified assumption that chronically elevated high-sensitivity cardiac troponin T (hs-cTnT) levels fluctuate randomly around a homeostatic set point.

Methods Two studies were conducted to assess biological hs-cTnT variation and to investigate the presence of a diurnal rhythm of hs-cTnT. Study 1 comprised 23 male subjects with type 2 diabetes, with no acute cardiovascular disease. Serial venous blood samples were drawn over an 11h period (8:30 AM to 7:30 PM). In study 2, presence of a diurnal hs-cTnT rhythm was investigated by hourly sampling of 7 subjects from study 1 over $25 \mathrm{~h}$.

Results In study 1 , we observed a gradual decrease in hs-cTnT values during the day (24 $\pm 2 \%)$. This decrease was present in all participants and was most prominent in subjects with the highest baseline hs-cTnT values (Pearson's $R=0.93$ ). Diurnal variation of hs-cTnT, as assessed in study 2 , was characterized by peak concentrations during morning hours (8:30 AM, $17.1 \pm 2.9 \mathrm{ng} / \mathrm{L})$, gradually decreasing values during daytime (8:30 PM, $11.9 \pm 1.6 \mathrm{ng} / \mathrm{L})$, and rising concentrations during nighttime (8:30 AM the next day, $16.9 \pm 2.8 \mathrm{ng} / \mathrm{L})$.

Conclusion A diurnal hs-cTnT rhythm substantiates the recommendation that all dynamic changes of hs-cTnT should be interpreted in relation to the clinical presentation. Epidemiological studies and risk-stratification protocols with the use of hs-cTnT may benefit from standardized sampling times. 


\section{Introduction}

Cardiac troponins (cTn) are sensitive markers of cardiac injury and the preferred biomarkers for the diagnosis or exclusion of acute myocardial infarction (AMI). Since the introduction of the high-sensitivity (hs) assays for cTn, detectable cTn levels are no longer restricted to cardiac patients, but can also be reliably assessed in apparently healthy individuals. Persistently elevated cTn concentrations near or above the $99^{\text {th }}$ percentile of a healthy reference population are frequently observed in patients with stable coronary artery disease, elderly subjects, and patients with various chronic diseases such as type 2 diabetes. ${ }^{1-4}$ Some have suggested that a higher decision limit threefold the $99^{\text {th }}$ percentile limit - might be advisable for diagnosing AMI in these patient groups. ${ }^{3,5}$

Regardless of the cut-off value that will be employed, the critical tool to discriminate between AMI-induced cTn release, and relatively stable hs-cTn elevations in chronic diseases, is serial testing and the assessment of kinetic changes of hs-cTn. Guidelines advocate a rise and/or fall of hs-cTn in patients with evidence of myocardial ischemia, with at least one hs-cTn value exceeding the $99^{\text {th }}$ percentile of the reference population. ${ }^{6}$ It should be recognized, however, that there is no perfect diagnostic threshold, and the application of any change criteria thus far is a trade-off, directed by the need for a high sensitivity or a high specificity. Therefore, a profound understanding of the naturally occurring biological variation of hs-cTn is essential when interpreting dynamic changes in serial testing. By definition, biological variation is measured by serially sampling healthy individuals at regular intervals. ${ }^{7}$ Thus far, studies in healthy individuals have reported short-term (hourly) biological variation of hs-cTn ranging from $3-48 \%$, which translates to reference change values (RCVs) of $45-85 \% .^{8-11}$ This finding means that a difference in serial hs-cTn measurements that exceeds this threshold, is unlikely due to analytical and biological variation only, and therefore considered a 'true' change at a p-value $<0.05$. However, it should be noted that healthy individuals may not be truly representative of the typical patient who requires clinical evaluation for chest pain.

The present study comprised a highly standardized assessment of within-day, diurnal and between-week biological variation of high-sensitivity cardiac troponin $\mathrm{T}$ (hscTnT) levels in subjects with increased odds of requiring hospital examination for chest pain. 


\section{Methods}

To examine within-day, diurnal and between-week variation in hs-cTnT levels, two distinct studies were conducted. Both studies complied with the Declaration of Helsinki and were approved by the institutional review board and the ethics committee at Maastricht University Medical Center. All participants provided written informed consent.

\section{Study 1: Within-day and between-week variation}

The study group consisted of 23 male subjects (body mass index [BMI] $30 \pm 4 \mathrm{~kg} / \mathrm{m}^{2}$; mean age $63 \pm 7$ years) who were diagnosed with type 2 diabetes for a mean duration of $7 \pm 5$ years (supplemental table 1). According to their BMI, 16 subjects were classified as overweight (BMI $25-30 \mathrm{~kg} / \mathrm{m}^{2}$ ) and 7 subjects as obese $\left(>30 \mathrm{~kg} / \mathrm{m}^{2}\right)$. Subjects were recruited by using advertisements in the local newspaper. Exclusion criteria were selfreported renal failure, liver disease, morbid obesity (BMI $>40 \mathrm{~kg} / \mathrm{m}^{2}$ ), history of severe cardiovascular problems (AMI or stroke in the past year), hypertension (>160 mm Hg systolic blood pressure and/or $>100 \mathrm{~mm} \mathrm{Hg}$ diastolic blood pressure) and exogenous insulin therapy. This study is part of a more extensive project investigating glycemic control and lifestyle interventions. ${ }^{12}$

Participants visited the laboratory by car or public transportation on 3 occasions (experiments A - C) in randomized order (figure 1). Subjects were asked to refrain from exhaustive physical labor and exercise training for 2 days before each test day. Experiment A estimated within-day hs-cTnT variation under sedentary conditions. From 8:30 AM till 7:30 PM, subjects were restricted to a sedentary laboratory environment and spent the day seated in a chair or couch, while reading, talking, watching television, or working on a laptop. Participants received standardized breakfast, lunch and dinner at 8:30 AM, 12:30 PM, and 5:00 PM. Blood samples ( $8 \mathrm{~mL})$ were collected from an antecubital venous catheter 5 min before each meal, as well as 90 and 150 min after each meal, resulting in a total of 9 blood samples collected within $11 \mathrm{~h}$.

Experiment B was conducted to investigate whether within-day hs-cTnT variation was affected by light physical activity. The test day was identical to experiment $A$, except for the addition of 15 min of slow-paced walking (total distance: 800 - 1,000 m, including 2 staircases) after each meal (at 9:15 AM, 1:15 PM and 5:45 PM) to mimic a day with low-to-moderate levels of physical activity. Experiment $\mathrm{C}$ was conducted to examine between-week variation of hs-cTnT. Before breakfast at 8:30 AM, a blood sample was collected. Between-week variation was calculated using the morning samples (8:30 AM) of experiments A through C. For all subjects, experiments $A$ through $C$ were performed at the same day of the week, within a total study period of $15-29$ days. 


\section{Diurnal variation}

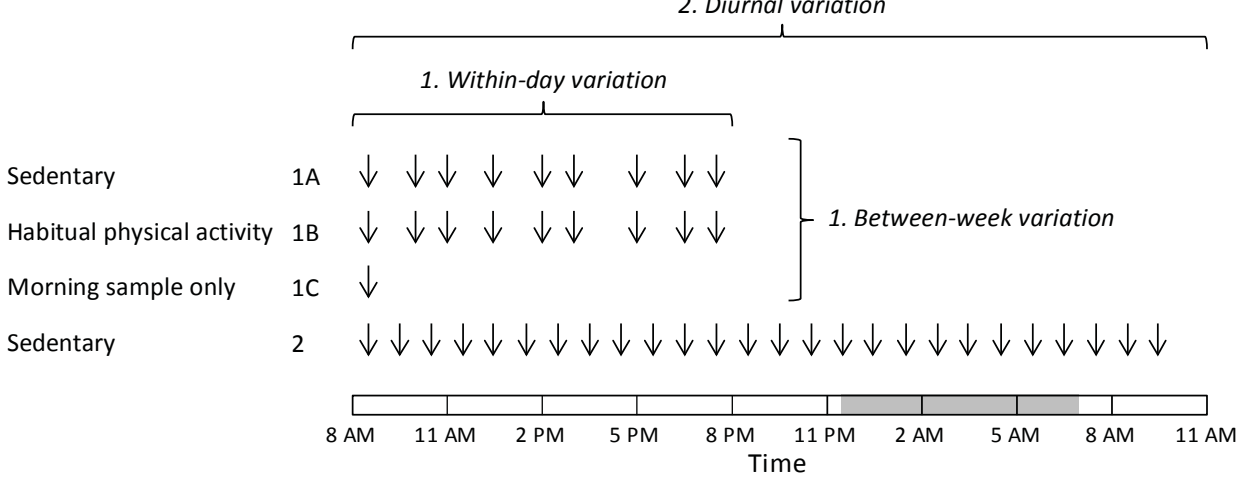

Figure 1. Overview of within-day, diurnal and between-week collection of blood samples.

Within-day variation was estimated under sedentary $(1 \mathrm{~A})$ or habitual physical activity conditions (1B) in 23 individuals over an $11 \mathrm{~h}$ period. Between-week variation was estimated by using the 3 morning samples (8:30 AM) collected at the same day of the week within a total study period of $15-29$ days (1A, 1B, and 1C). Diurnal changes in hs-cTnT were monitored in 7 participants under sedentary conditions (2). Participants slept in supine position between 11:30 PM and 7:00 AM (gray area). All blood samples (indicated by arrows) were collected at standardized clock hours.

\section{Study 2: Diurnal variation}

After finalizing study 1 , additional experiments were conducted to investigate the hypothesis that circulating hs-cTnT exhibits a diurnal rhythm. Seven subjects who also participated in study 1 were sampled every hour $(8 \mathrm{~mL})$ by using an antecubital venous catheter over a time span of $25 \mathrm{~h}$ (figure 1). Participants were restricted to a sedentary laboratory environment from 8:30 AM till 9:30 AM the next day with standardized meals consumed at 8:30 AM, 12:30 PM and 5:00 PM (breakfast, lunch and dinner, respectively). Subjects went to bed at 11:30 PM and the lights were off at 11:35 PM until 7:00 AM. During the night, polyethylene coiled extension lines (Vygon) were used for blood sampling to prevent disturbance of participants' sleep.

\section{Laboratory measurements}

Blood samples were collected in EDTA-containing tubes. Immediately upon collection, samples were centrifuged and plasma was stored at $-80^{\circ} \mathrm{C}$ until analysis. Hematology parameters were analyzed immediately in the diurnal samples on a Sysmex XE-5000 analyzer (Sysmex Corporation). cTnT was measured in duplicate using a high-sensitivity cTnT assay (Roche Diagnostics) on a Cobas 6000 analyzer (lot number 167650). The limits of blank and detection of the assay were 3 and $5 \mathrm{ng} / \mathrm{L}$, respectively; the $99^{\text {th }}$ percentile among healthy subjects is $14 \mathrm{ng} / \mathrm{L} .{ }^{13}$ Creatinine, creatine kinase (CK) and albumin were measured on a Cobas 6000 analyzer (Roche). The estimated glomerular filtration rate (eGFR) was calculated according to Chronic Kidney Disease-Epidemiology Collaboration formula. ${ }^{14}$ 


\section{Statistical analysis}

Between-person $\left(\mathrm{CV}_{\mathrm{G}}\right)$, within-person biological $\left(\mathrm{CV}_{\mathrm{i}}\right)$ and analytical $\left(\mathrm{CV}_{\mathrm{A}}\right)$ coefficients of variation were calculated by using a balanced analysis of variance with a nested random design in 2 levels; 95\% confidence intervals (Cls) of these estimated variance components were calculated according to Burdick and Graybill. ${ }^{15}$ Outliers in terms of the $\mathrm{CV}_{\mathrm{i}}$ were identified by Cochran's C test. RCVs (Z-score of 1.96) and the index of individuality were calculated according to Fraser and Harris. ${ }^{16}$ RCVs were also evaluated after a lognormal transformation. ${ }^{17}$ The Wilcoxon signed-rank test was applied to compare hscTnT concentrations measured at 8:30 AM and 7:30 PM. Hemoglobin and hematocrit values were used to quantify possible plasma volume changes due to changes in hydration status and/or posture during the diurnal variation study. ${ }^{18}$

Diurnal hs-cTnT changes were analyzed by fitting individual data to a cosine curve with a $24 \mathrm{~h}$ period by using the method of cosinor-rhythmometry as described by Nelson et.al. ${ }^{19}$ Briefly, the cosinor model is described as: $Z(t)=M+A \cdot \cos (\omega t+\phi)+e(t)$, where $Z(t)$ represents the measured hs-cTnT concentration at a given time $(t), M$ the mesor (average value of a cosine curve fitted to the data), A the amplitude (one-half the difference between the peak and the nadir value), $\omega$ the angular frequency (degrees per unit time, with $360^{\circ}$ representing a complete cycle), $\phi$ the acrophase (timing of maximal value in degrees) and $\mathrm{e}(\mathrm{t})$ the error between the cosinor model and the measurement. Rearrangement of the model using trigonometric identities produces a linear model in the coefficients. Evidence of a diurnal rhythm was indicated by a significant cosinor model fit $(p<0.05)$. A mean cosinor model was computed by using the rearranged cosine models in a linear mixed model with a random subject term. All statistical calculations were performed using SPSS version 20 (IBM SPSS Statistics).

\section{Results}

Within-day biological cTnT variation is larger than between-week variation The within-day distribution of hs-cTnT (8:30 AM - 7:30 PM) in 23 subjects under sedentary conditions is shown in figure $2 A$. All values (range: $5.2-29.5 \mathrm{ng} / \mathrm{L}$ ) were above the detection limit of the assay, and 6 subjects had at least 1 measurement above the $99^{\text {th }}$ percentile of $14 \mathrm{ng} / \mathrm{L}$. To verify the results obtained under completely sedentary conditions, the same subjects were also measured during a second test day at a 1-week interval that included habitual physical activity (see methods). Results were highly comparable to the sedentary test day: hs-cTnT levels ranged from $5.4-31.2 \mathrm{ng} / \mathrm{L}$, and 9 subjects had at least 1 measurement above the $99^{\text {th }}$ percentile (figure $2 B$ ). To estimate between-week variability of hs-cTnT, an additional morning sample (8:30 AM) was collected at another 1-week interval. hs-cTnT levels on 3 separate visits were between 
6.0 and $34.1 \mathrm{ng} / \mathrm{L}$ (figure 2C). Eight subjects had at least one 8:30 AM measurement above the $99^{\text {th }}$ percentile.

A

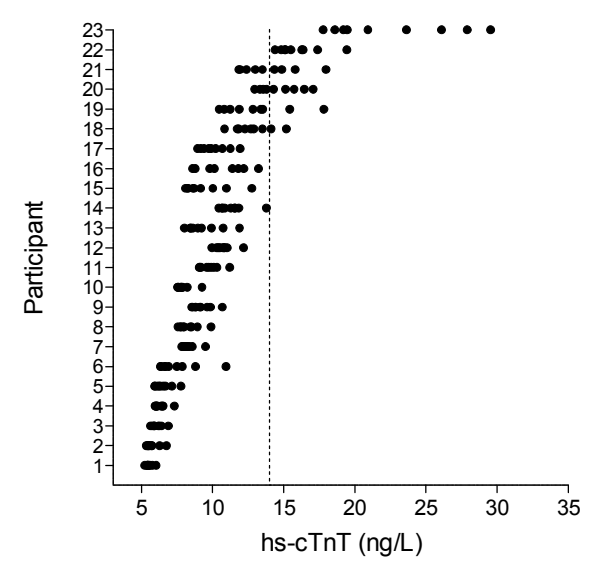

B

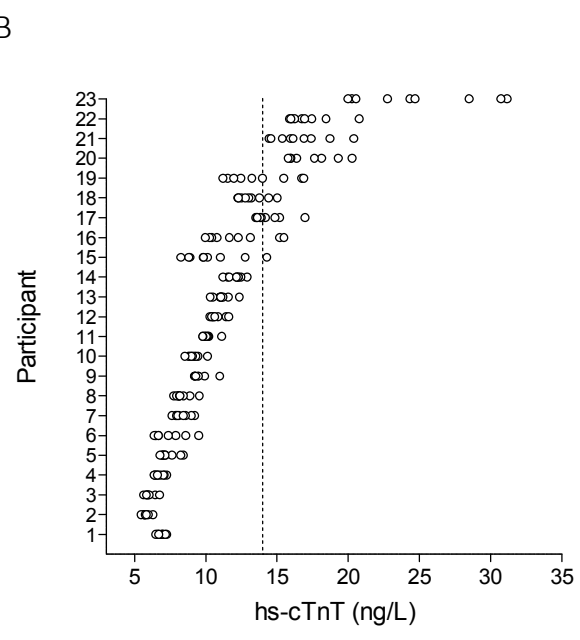

C

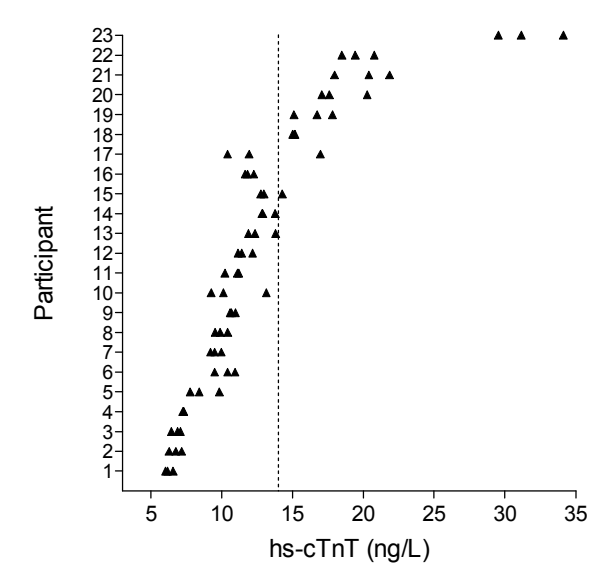

Figure 2. Within-day and between-week distribution of hs-cTnT concentrations.

Within-day distribution of hs-cTnT (8:30 AM 7:30 PM) under A. sedentary conditions and B. standardized habitual physical activity. C. Between-week distribution of hs-cTnT assessed by 3 morning samples (8:30 AM). Symbols represent mean hs-cTnT values. The $99^{\text {th }}$ percentile (14 $\mathrm{ng} / \mathrm{L}$ ) is indicated by the dotted line. Subjects are ranked based on their visual range of hs-cTnT level during the sedentary test day: generally, high numbers correspond to a wider hs-cTnT range. The ranking used for figure $2 \mathrm{~A}$ is extended to figure $2 \mathrm{~B}, 2 \mathrm{C}$ and supplemental figure 1 .

Table 1 lists the within-day and between-week hs-cTnT variation components and their $95 \% \mathrm{Cls}$. The power of this study to detect a $\mathrm{CV}_{i}$ different from 0 was $>0.8$, taking into account the number of subjects, samples, and replicates and the observed ratio between the analytical variation and the biological variation. ${ }^{20}$ Within-day biological variability of hs-cTnT was not affected by habitual physical activity $\left(\mathrm{CV}_{i} 14.2 \%\right.$ [95\% Cl: 12.9 - 15.9\%] under sedentary conditions compared with $\mathrm{CV}_{\mathrm{i}} 12.7 \%$ [95\% Cl: 11.5 14.2\%] under habitual physical activity). Surprisingly, the within-day biological variation of hs-cTnT under sedentary conditions was significantly larger than the between-week 


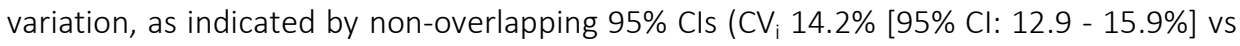
$\mathrm{CV}_{i}$ 9.7\% [95\% Cl: 8.1 - 12.3\%]). A similar trend was present between within-day biological variation of hs-cTnT under habitual physical activity and between-week biological variation. Identification and exclusion of subjects who were considered outliers in terms of their within-person biological variation provided similar results (data not shown). We further examined the individual pattern of hs-cTnT during the day to gain additional insight in the relatively high within-day biological variation of hs-cTnT.

Table 1. Within-day and between-week variation in hs-cTnT levels.

\begin{tabular}{|c|c|c|c|}
\hline & \multicolumn{2}{|l|}{ Within-day } & \multirow[t]{2}{*}{ Between-week } \\
\hline & Sedentary & Habitual physical activity & \\
\hline Samples / subjects, $\mathrm{n}$ & $207 / 23$ & $207 / 23$ & $69 / 23$ \\
\hline Mean levels, ng/L & 10.3 & 11.3 & 12.8 \\
\hline \multicolumn{4}{|l|}{ Variance components } \\
\hline $\mathrm{CV}_{\mathrm{G}}, \%(95 \% \mathrm{Cl})$ & $38.4(29.5-54.5)$ & $40.4(31.1-57.4)$ & $43.8(33.6-62.2)$ \\
\hline$C V_{i}, \%(95 \% C l)$ & $14.2(12.9-15.9)$ & $12.7(11.5-14.2)$ & $9.7(8.1-12.3)$ \\
\hline $\mathrm{CV}_{\mathrm{A}},{ }^{\mathrm{a}} \%(95 \% \mathrm{Cl})$ & $2.0(1.8-2.2)$ & $1.9(1.8-2.1)$ & $1.8(1.5-2.1)$ \\
\hline \multicolumn{4}{|l|}{$\mathrm{RCV}^{\mathrm{b}}$} \\
\hline Normal, \% & 39.8 & 35.7 & 27.4 \\
\hline Log-normal, \% & $+48.6,-32.7$ & $+42.7,-29.9$ & $+31.4,-23.9$ \\
\hline Index of individuality & 0.37 & 0.32 & 0.23 \\
\hline
\end{tabular}

${ }^{a}$ On the basis of duplicate measurements. ${ }^{b}$ On the basis of a Z-score of 1.96 .

$\mathrm{Cl}$; confidence interval, $\mathrm{CV}_{\mathrm{A}}$; analytical coefficient of variation, $\mathrm{CV}_{\mathrm{G}}$; between-person coefficient of variation, $\mathrm{CV}_{\mathrm{i}}$; within-person biological coefficient of variation, $\mathrm{RCV}$; reference change value.

hs-cTnT concentrations exhibit a gradual decrease during the day

hs-cTnT concentrations exhibited a gradual decrease during the sedentary test day, with the highest concentration at 8:30 AM (median $11.8 \mathrm{ng} / \mathrm{L}$, interquartile range [IQR]: 9.3 $15.2 \mathrm{ng} / \mathrm{L}$ ) and the lowest concentration at the end of the test day (7:30 PM; median 8.6 ng/L [IQR: 6.7 - $10.8 \mathrm{ng} / \mathrm{L}$; $\mathrm{p}$ <0.001) (mean hs-cTnT decrease of 24\%, figure 3A). A similar decrease has been previously reported for CK levels under constant resting conditions, and this decrease could be reversed to stable concentrations when light physical activity was introduced during the day. ${ }^{21}$ To investigate whether the same mechanism underlies the continuous decrease of hs-cTnT, we examined the hs-cTnT pattern during the second test day, which included 3 short walking sessions. Figure $3 \mathrm{~A}$ shows that the decline of hs-cTnT was only marginally affected by 3 walking sessions during the day: a median of $11.4 \mathrm{ng} / \mathrm{L}$ (IQR: 9.2 - $16.7 \mathrm{ng} / \mathrm{L}$ ) at 8:30 AM and a median of $9.8 \mathrm{ng} / \mathrm{L}$ (IQR: 6.8 - $12.3 \mathrm{ng} / \mathrm{L}$ ) at 7:30 PM ( $\mathrm{p}<0.001$ ) (mean hs-cTnT decrease of 18\%). In 
contrast, the gradually decreasing pattern of CK observed under sedentary conditions was completely reversed by habitual physical activity (figure 3B), consistent with previous observations. ${ }^{21}$ The decreasing pattern of hs-cTnT was an evident phenomenon in all subjects, and it was most prominent in subjects with the highest basal hs-cTnT levels (figure 3C, supplemental figure 1).

A

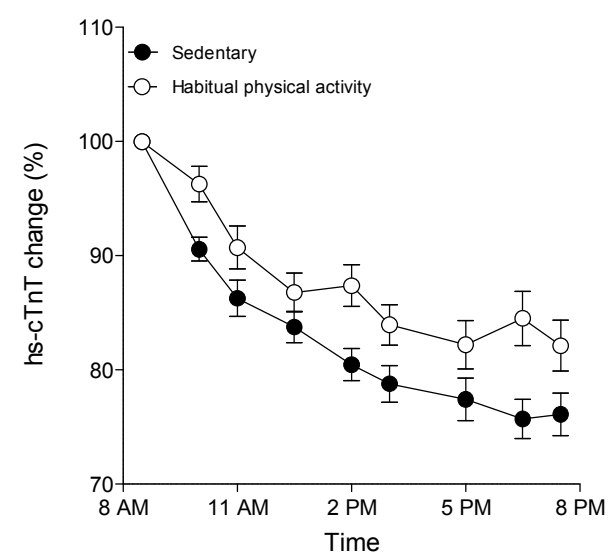

B

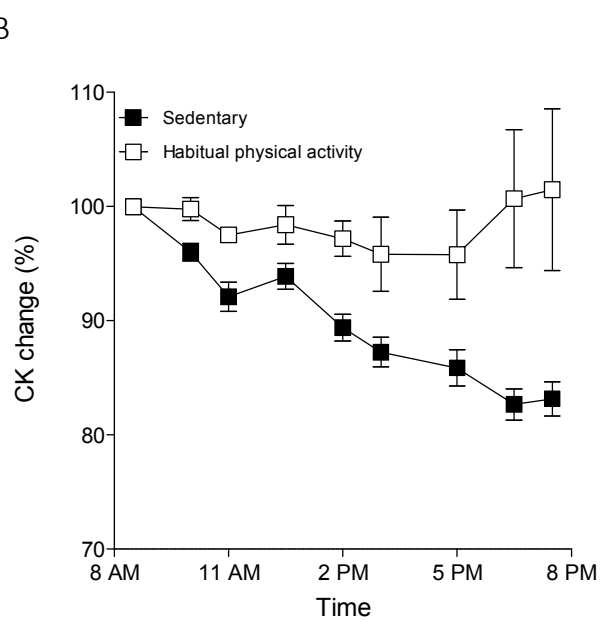

C

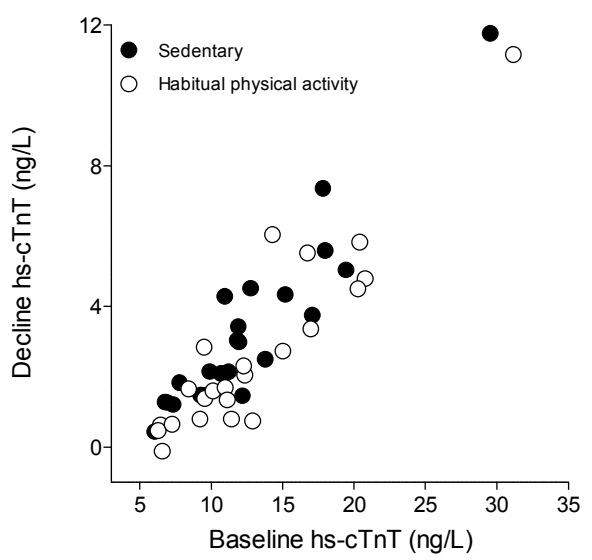

Figure 3. hs-cTnT exhibits a gradual decrease during the day, which is not affected by habitual physical activity.

A. hs-cTnT change from baseline value under sedentary and habitual physical activity conditions (mean $\pm \mathrm{SEM}$ ). B. Creatine kinase (CK) change from baseline value under sedentary conditions and habitual physical activity (mean \pm SEM). C. Correlation between baseline hs-cTnT value and magnitude of hs-cTnT decrease under sedentary conditions and habitual physical activity (Pearson's R: 0.93 and 0.91 , both $p<0.001$ ). 


\section{Circulating hs-cTnT exhibits a diurnal rhythm}

To investigate whether the decrease in hs-cTnT during daytime is followed by a rise during night hours, we collected blood samples from 7 subjects every hour over a time span of 25h (from 8:30 AM - 9:30 AM the next day). In all participants, the hs-cTnT decline during daytime was accompanied by an increase during night hours, with peak concentrations in the morning (figure $4 A$ ). The intraindividual difference between the peak and the nadir hs-cTnT concentrations ranged from $3.4-11.8 \mathrm{ng} / \mathrm{L}$. Correction for posture-induced changes in plasma volume did not abrogate the diurnal rhythm (data not shown). ${ }^{18}$ Consistent with the sedentary test day, CK values decreased continuously throughout the $25 \mathrm{~h}$ period, whereas eGFR and albumin levels fluctuated randomly around the subject's homeostatic set point (data not shown).

A

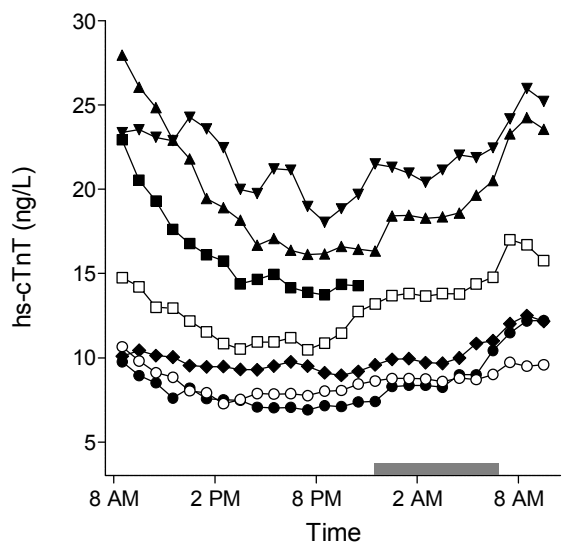

B

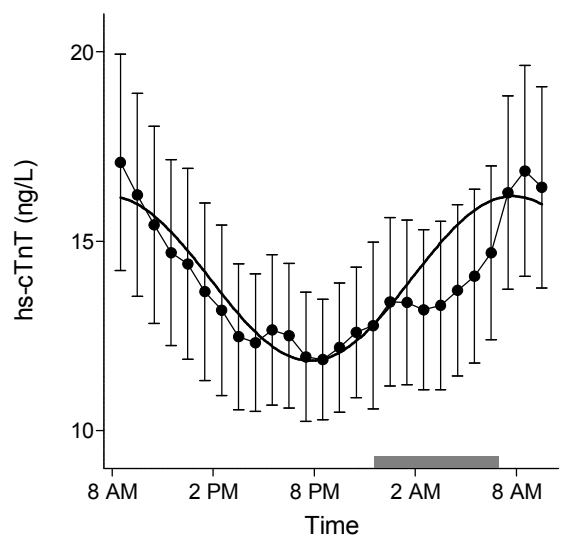

Figure 4. hs-cTnT exhibits a diurnal rhythm.

A. Individual $25 \mathrm{~h}$ hs-cTnT profiles under sedentary conditions. Subjects slept in supine position between 11:30 PM and 7:00 AM (gray area). Sampling in one subject (closed squares) was terminated at 10:30 PM due to technical problems with the intravenous cannula that hindered sampling throughout the night. B. Mean \pm SEM 25h hs-cTnT profile and fitted cosine curve.

On the basis of this oscillation in hs-cTnT, we fitted individual hs-cTnT data to a cosine function to model the variation in the observed hs-cTnT concentration as a function of time. The fitted cosine function for each individual can be described by the mesor, amplitude and acrophase (table 2). Quantitatively, in all participants, the diurnal hs-cTnT variation was significantly described by the fitted cosine curve, as indicated by the 'goodness-of-fit' statistic ( $R^{2}$ range: 0.55 - 0.91, all $p<0.001$ ). In line with the individual hs-cTnT curves, the group cosinor model exhibited a strong fit with the mean diurnal hs-cTnT profile (figure 4B). 
Table 2. The individual diurnal variation of hs-cTnT is significantly described by a cosine curve.

\begin{tabular}{lllll}
\hline Subject $^{\mathrm{a}}$ & $\begin{array}{l}\text { Mesor, } \\
\mathrm{ng} / \mathrm{L}\end{array}$ & $\begin{array}{l}\text { Amplitude, } \\
\mathrm{ng} / \mathrm{L}\end{array}$ & $\begin{array}{l}\text { Acrophase, } \\
\mathrm{h}\end{array}$ \\
\hline $\boldsymbol{\Delta}$ & 19.5 & 4.1 & $9: 08 \mathrm{AM}$ & $0.80^{*}$ \\
$\boldsymbol{\nabla}$ & 21.7 & 2.3 & $9: 18 \mathrm{AM}$ & $0.74^{*}$ \\
$\boldsymbol{\square}^{\mathrm{b}}$ & 17.5 & 4.0 & $6: 37 \mathrm{AM}$ & $0.91^{*}$ \\
$\square$ & 12.9 & 2.4 & $6: 03 \mathrm{AM}$ & $0.81^{*}$ \\
0 & 8.6 & 0.9 & $6: 30 \mathrm{AM}$ & $0.65^{*}$ \\
$\boldsymbol{0}$ & 10.0 & 1.0 & $7: 35 \mathrm{AM}$ & $0.55^{*}$ \\
\hline
\end{tabular}

${ }^{\mathrm{a}}$ Subject symbols correspond to the symbols used in figure 4A. ${ }^{\mathrm{b}}$ Sample collection until 10:30 PM (14h). ${ }^{*} \mathrm{p}$ $<0.001$.

\section{Discussion}

The present study provides novel evidence that circulating hs-cTnT levels exhibit a diurnal rhythm, characterized by peak concentrations during morning hours, gradually decreasing concentrations throughout daytime, and rising concentrations during nighttime. This diurnal oscillation is a general phenomenon, but was most prominent in subjects with the highest baseline hs-cTnT values.

\section{hs-cTnT and type 2 diabetes}

We studied biological variability of hs-cTnT in patients with type 2 diabetes without acute cardiovascular disease. Twenty percent of patients with type 2 diabetes have persistently elevated hs-cTnT concentrations above the $99^{\text {th }}$ percentile, in the absence of an acute cardiac event. ${ }^{22,23}$ Even in the pre-diabetic state (impaired glucose tolerance or impaired fasting glucose), hs-cTnT elevations are frequently observed. ${ }^{23}$ Type 2 diabetes is a highly prevalent condition (25\%) among patients who present at the emergency department with symptoms suggestive of AMI. ${ }^{5,24}$ A thorough understanding of the biological variation of hs-cTnT levels near or above the $99^{\text {th }}$ percentile is therefore a timely, and relatively unexplored diagnostic issue.

\section{Diurnal oscillation of hs-cTnT: effect on reference change values}

The extended timeline and highly standardized protocol for blood sampling formed the basis for the key finding of this study: the presence of a diurnal rhythm of hs-cTnT. A remarkable consequence of the diurnal rhythm is that the within-day biological variation was significantly higher than the between-week biological variation, provided that the latter was measured in samples collected at the same hour of the day. Another 
important consequence of the diurnal rhythm relates to the violation of an important precondition when using RCV; that is, the assumption that biological variation is random. This point can be illustrated using data from figure 4. Under conditions of nonrandom variation (e.g. a diurnal rhythm), the calculated biological variation strongly depends on the time frame chosen. For example, when a time frame between 8:30 AM and 12:30 PM is considered, the calculated biological variation is more than twice the biological variation assessed in the time frame between 4:30 PM and 8:30 PM ( $\mathrm{CV}_{i}$ 9.4\% [95\% Cl: 7.4 - 12.7\%] vs. $\mathrm{CV}_{\mathrm{i}} 4.3 \%$ [95\% Cl: 3.1 - 6.2\%], respectively). These differences translate directly to changes of similar magnitude for RCV. The use of different time frames across studies might have contributed to a relatively broad range of biological variations in hs-cTn which have been previously assessed in healthy subjects and patients with stable cardiovascular disease. ${ }^{8-11,25,26}$ Altogether, the observation of a diurnal rhythm of hs-cTnT, with the highest amplitude in patients with the highest baseline hscTnT levels, may pose an additional challenge to diagnose AMI in this patient group.

\section{Circadian variation in cardiovascular physiology and pathology}

The finding of a cardiac biomarker exhibiting rhythmic activity is in line with described circadian variation in cardiovascular physiology, gene and/or protein expression. ${ }^{27}$ For example, heart rate and blood pressure are lowest at nighttime and begin to rise before the time of awakening in anticipation of the demands of daytime activities and they decrease again in the evening, anticipating sleep. ${ }^{28}$ In addition, it has been shown that enhanced amino acid incorporation into rat myocardial tissue takes place during the sleeping hours, with the least synthesis occurring $12 \mathrm{~h}$ later, indicating that myocardial growth and renewal processes occur especially during the night. ${ }^{29}$ In addition to physiology, cardiovascular pathology also exhibits diurnal variation. For example, the incidence of AMI displays a daily rhythmic pattern with the highest incidence between 6:00 AM and 12:00 PM. ${ }^{30,31}$ Even infarct-size exhibits a circadian dependence on the time-ofday onset of ischemia, with the largest infarct-sizes observed in patients with symptom onset in the early morning hours. ${ }^{32,33}$

\section{Biological significance of a diurnal hs-cTnT rhythm}

The biological significance of a diurnal pattern in hs-cTnT has yet to be established. Related to this matter is elucidation of the release mechanism of hs-cTnT, as well as clearance from the bloodstream. Similar to findings in the literature, ${ }^{34}$ eGFR was stable during the $25 \mathrm{~h}$ period, indicating that the fluctuations in hs-cTnT could not be explained by intraindividual changes in eGFR. However, it is not clear if the fluctuations reflect a disease-specific mechanism (e.g. ischemia) or a physiological mechanism (e.g. protein turnover). Nevertheless, these findings provide support for the hypothesis that hs-cTn can be applied to track the impact of a number of physiological and pathophysiological 
cardiovascular processes that are potentially unrelated to cardiac ischemia. ${ }^{35,36}$ Therefore, it would be of interest to assess the rhythmicity of hs-cTnT in healthy individuals. These studies will become feasible when even more sensitive assays become available, with higher precision in the lower range.

\section{Study limitations}

Because the study group consisted of overweight or obese type 2 diabetic patients treated with oral glucose-lowering medication, we cannot generalize these results directly to the cardio-healthy population. In addition, various factors specific for the studied group might have contributed to the diurnal rhythm of hs-cTnT. The effect of antidiabetic medication on hs-cTnT levels is unknown, although medication was stable for at least 3 months before patient inclusion and was continued as normal during the entire study period, including test days. We did not explicitly control for intermittent low oxygen saturation during the night (e.g. sleep apnea), which is often associated with subjects who are overweight or obese. In addition, the relationship between diurnal variation in heart rate and blood pressure and hs-cTnT levels has not been explored. Finally, sample size, particularly for evaluation of diurnal variation $(n=7)$, was relatively low to exclude sample size error. However, the validity of the data is supported by the high consistency of the observations across all participants.

\section{Conclusions}

hs-cTnT exhibited a diurnal rhythm in subjects with increased odds of requiring hospital examination for chest pain. The presence of a diurnal rhythm has direct consequences for epidemiological studies and stresses the necessity of interpreting all dynamic changes of hs-cTnT in relation to the clinical presentation. Although the finding of a biomarker exhibiting circadian variation is in line with cardiovascular physiology and pathology, further studies are needed to understand the biological significance and to demonstrate the rhythmicity in various disease populations.

\section{Acknowledgements}

This study was supported by a research grant from Stichting De Weijerhorst to M.v.D.-V. 


\section{References}

1. Everett BM, Cook NR, Magnone MC, et al. Sensitive cardiac troponin T assay and the risk of incident cardiovascular disease in women with and without diabetes mellitus: the Women's Health Study. Circulation. 2011;123(24):2811-2818.

2. Omland T, de Lemos JA, Sabatine MS, et al. A sensitive cardiac troponin T assay in stable coronary artery disease. N Engl J Med. 2009;361(26):2538-2547.

3. Thygesen K, Mair J, Giannitsis E, et al. How to use high-sensitivity cardiac troponins in acute cardiac care. Eur Heart J. 2012;33(18):2252-2257.

4. defilippi $\mathrm{CR}$, de Lemos JA, Christenson $\mathrm{RH}$, et al. Association of serial measures of cardiac troponin T using a sensitive assay with incident heart failure and cardiovascular mortality in older adults. JAMA. 2010;304(22):2494-2502.

5. Mueller M, Biener $\mathrm{M}$, Vafaie $\mathrm{M}$, et al. Absolute and relative kinetic changes of high-sensitivity cardiac troponin $T$ in acute coronary syndrome and in patients with increased troponin in the absence of acute coronary syndrome. Clin Chem. 2012;58(1):209-218.

6. Thygesen K, Alpert JS, Jaffe AS, et al. Third universal definition of myocardial infarction. Eur Heart J. 2012;33(20):2551-2567.

7. Fraser CG. The Nature of Biological Variation. In: Biological variation: from principles to practice: AACC Press; 2001:1-27.

8. Wu AH, Lu QA, Todd J, Moecks J, Wians F. Short- and long-term biological variation in cardiac troponin I measured with a high-sensitivity assay: implications for clinical practice. Clin Chem. 2009;55(1):52-58.

9. Vasile VC, Saenger AK, Kroning JM, Jaffe AS. Biological and analytical variability of a novel high-sensitivity cardiac troponin T assay. Clin Chem. 2010;56(7):1086-1090.

10. Vasile VC, Saenger AK, Kroning JM, Klee GG, Jaffe AS. Biologic variation of a novel cardiac troponin I assay. Clin Chem. 2011;57(7):1080-1081.

11. Frankenstein L, Wu AH, Hallermayer K, Wians FH, Jr., Giannitsis E, Katus HA. Biological variation and reference change value of high-sensitivity troponin $T$ in healthy individuals during short and intermediate follow-up periods. Clin Chem. 2011;57(7):1068-1071.

12. van Dijk JW, Venema M, van Mechelen W, Stehouwer CD, Hartgens F, van Loon LJ. Effect of moderateintensity exercise versus activities of daily living on 24-hour blood glucose homeostasis in male patients with type 2 diabetes. Diabetes Care. 2013;36(11):3448-3453.

13. Giannitsis E, Kurz K, Hallermayer K, Jarausch J, Jaffe AS, Katus HA. Analytical validation of a highsensitivity cardiac troponin T assay. Clin Chem. 2010;56(2):254-261.

14. Levey AS, Stevens LA, Schmid CH, et al. A new equation to estimate glomerular filtration rate. Ann Intern Med. 2009;150(9):604-612.

15. Burdick RK, Graybill FA. Confidence intervals on variance components. New York: Marcel Dekker; 1992.

16. Fraser CG, Harris EK. Generation and application of data on biological variation in clinical chemistry. Crit Rev Clin Lab Sci. 1989;27(5):409-437.

17. Fokkema MR, Herrmann Z, Muskiet FA, Moecks J. Reference change values for brain natriuretic peptides revisited. Clin Chem. 2006;52(8):1602-1603.

18. Dill DB, Costill DL. Calculation of percentage changes in volumes of blood, plasma, and red cells in dehydration. J Appl Physiol. 1974;37(2):247-248.

19. Nelson W, Tong YL, Lee JK, Halberg F. Methods for cosinor-rhythmometry. Chronobiologia. 1979;6(4):305-323.

20. Roraas T, Petersen PH, Sandberg S. Confidence intervals and power calculations for within-person biological variation: effect of analytical imprecision, number of replicates, number of samples, and number of individuals. Clin Chem. 2012;58(9):1306-1313.

21. Gutenbrunner C. Circadian variations of the serum creatine kinase level - a masking effect? Chronobiol Int. 2000;17(4):583-590.

22. Hallen J, Johansen OE, Birkeland KI, et al. Determinants and prognostic implications of cardiac troponin T measured by a sensitive assay in type 2 diabetes mellitus. Cardiovasc Diabetol. 2010;9:52.

23. Zheng J, Ye P, Luo L, Xiao W, Xu R, Wu H. Association between blood glucose levels and high-sensitivity cardiac troponin $\mathrm{T}$ in an overt cardiovascular disease-free community-based study. Diabetes Res Clin Pract. 2012;97(1):139-145. 
24. Reichlin T, Hochholzer W, Bassetti S, et al. Early diagnosis of myocardial infarction with sensitive cardiac troponin assays. N Engl J Med. 2009;361(9):858-867.

25. Nordenskjold AM, Ahlstrom H, Eggers KM, et al. Short- and long-term individual variation in cardiac troponin in patients with stable coronary artery disease. Clin Chem. 2013;59(2):401-409.

26. Scharnhorst V, Krasznai K, van 't Veer M, Michels RH. Variation of cardiac troponin I and T measured with sensitive assays in emergency department patients with noncardiac chest pain. Clin Chem. 2012;58(8):1208-1214.

27. Martino TA, Sole MJ. Molecular time: an often overlooked dimension to cardiovascular disease. Circ Res. 2009;105(11):1047-1061.

28. Degaute JP, van de Borne P, Linkowski P, Van Cauter E. Quantitative analysis of the 24-hour blood pressure and heart rate patterns in young men. Hypertension. 1991;18(2):199-210.

29. Rau E, Meyer DK. A diurnal rhythm of incorporation of $L-[3 \mathrm{H}]$ leucine in myocardium of the rat. Recent Adv Stud Cardiac Struct Metab. 1975;7:105-110.

30. Cohen MC, Rohtla KM, Lavery CE, Muller JE, Mittleman MA. Meta-analysis of the morning excess of acute myocardial infarction and sudden cardiac death. Am J Cardiol. 1997;79(11):1512-1516.

31. Muller JE, Stone PH, Turi ZG, et al. Circadian variation in the frequency of onset of acute myocardial infarction. N Engl J Med. 1985;313(21):1315-1322.

32. Reiter R, Swingen C, Moore L, Henry TD, Traverse JH. Circadian dependence of infarct size and left ventricular function after ST elevation myocardial infarction. Circ Res. 2012;110(1):105-110.

33. Fournier S, Eeckhout E, Mangiacapra F, et al. Circadian variations of ischemic burden among patients with myocardial infarction undergoing primary percutaneous coronary intervention. Am Heart J. 2012;163(2):208-213.

34. Larsson A, Akerstedt T, Hansson LO, Axelsson J. Circadian variability of cystatin C, creatinine, and glomerular filtration rate (GFR) in healthy men during normal sleep and after an acute shift of sleep. Chronobiol Int. 2008;25(6):1047-1061.

35. defilippi CR, de Lemos JA, Tkaczuk AT, et al. Physical activity, change in biomarkers of myocardial stress and injury, and subsequent heart failure risk in older adults. J Am Coll Cardiol. 2012;60(24):2539-2547.

36. Lazzarino Al, Hamer M, Gaze D, Collinson P, Steptoe A. The association between cortisol response to mental stress and high-sensitivity cardiac troponin T plasma concentration in healthy adults. J Am Coll Cardiol. 2013;62(18):1694-1701. 


\section{Supplemental information}

\section{Supplemental tables}

Supplemental table 1. Participants' characteristics.

\begin{tabular}{|c|c|}
\hline Characteristic & $\begin{array}{l}\text { All } \\
n=23\end{array}$ \\
\hline Age, years & $63 \pm 7$ \\
\hline $\mathrm{BMI}, \mathrm{kg} / \mathrm{m}^{2}$ & $30 \pm 4$ \\
\hline Systolic blood pressure, mm Hg & $138 \pm 15$ \\
\hline Diastolic blood pressure, $\mathrm{mm} \mathrm{Hg}$ & $74 \pm 10$ \\
\hline Time since diagnosis of type 2 diabetes, years & $7 \pm 5$ \\
\hline hs-cTnT, ${ }^{a}$ ng/L & $12(9-15)$ \\
\hline $\mathrm{CK},{ }^{\mathrm{a}} \mathrm{U} / \mathrm{L}$ & $82(71-103)$ \\
\hline Albumin, ${ }^{a} \mathrm{~g} / \mathrm{L}$ & $44(43-46)$ \\
\hline $\mathrm{eGFR},{ }^{\mathrm{ab}} \mathrm{mL} / \mathrm{min} / 1.73 \mathrm{~m}^{2}$ & $89(81-94)$ \\
\hline \multicolumn{2}{|l|}{ Medication, ${ }^{c} n$} \\
\hline Oral blood glucose-lowering medication & 22 \\
\hline Metformin + SUD & 4 \\
\hline Metformin + DPP4 inhibitor & 2 \\
\hline Metformin + SUD + DPP4 inhibitor & 1 \\
\hline Metformin + TZD & 2 \\
\hline Metformin only & 13 \\
\hline Lipid-lowering medication & 18 \\
\hline Anti-hypertensive medication & 3 \\
\hline
\end{tabular}

Data is presented as $n$, mean \pm SD or median (IQR).

${ }^{a}$ Value at baseline (8:30 AM) of visit A. ${ }^{b}$ On the basis of the CKD-EPI formula. ${ }^{14}{ }^{\mathrm{c}}$ Medication status was stable for at least 3 months prior to inclusion, and continued as normal during the study period, including test days. CK; creatine kinase, DPP4; dipeptidyl peptidase-4, eGFR; estimated glomerular filtration rate, SUD; sulfonylurea derivate, TZD; thiazolidinedione. 


\section{Supplemental figures}
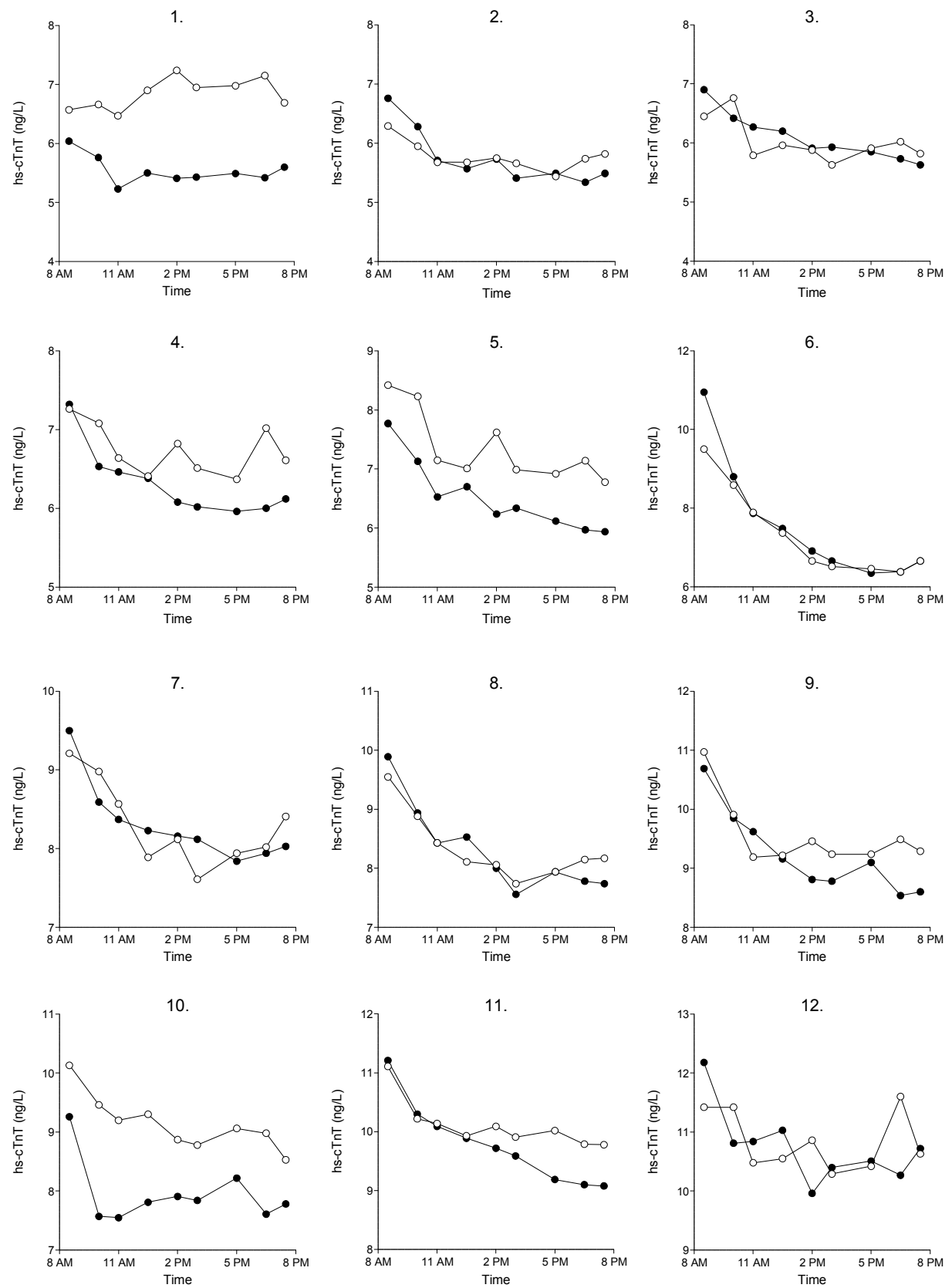

Supplemental figure 1. Individual hs-cTnT profiles during the day (continued on next page).

hs-cTnT levels were measured under sedentary (black circle) and habitual physical activity (white circle) conditions. Depicted are the mean of duplicate measurements. The numbering is identical to figure 2. 

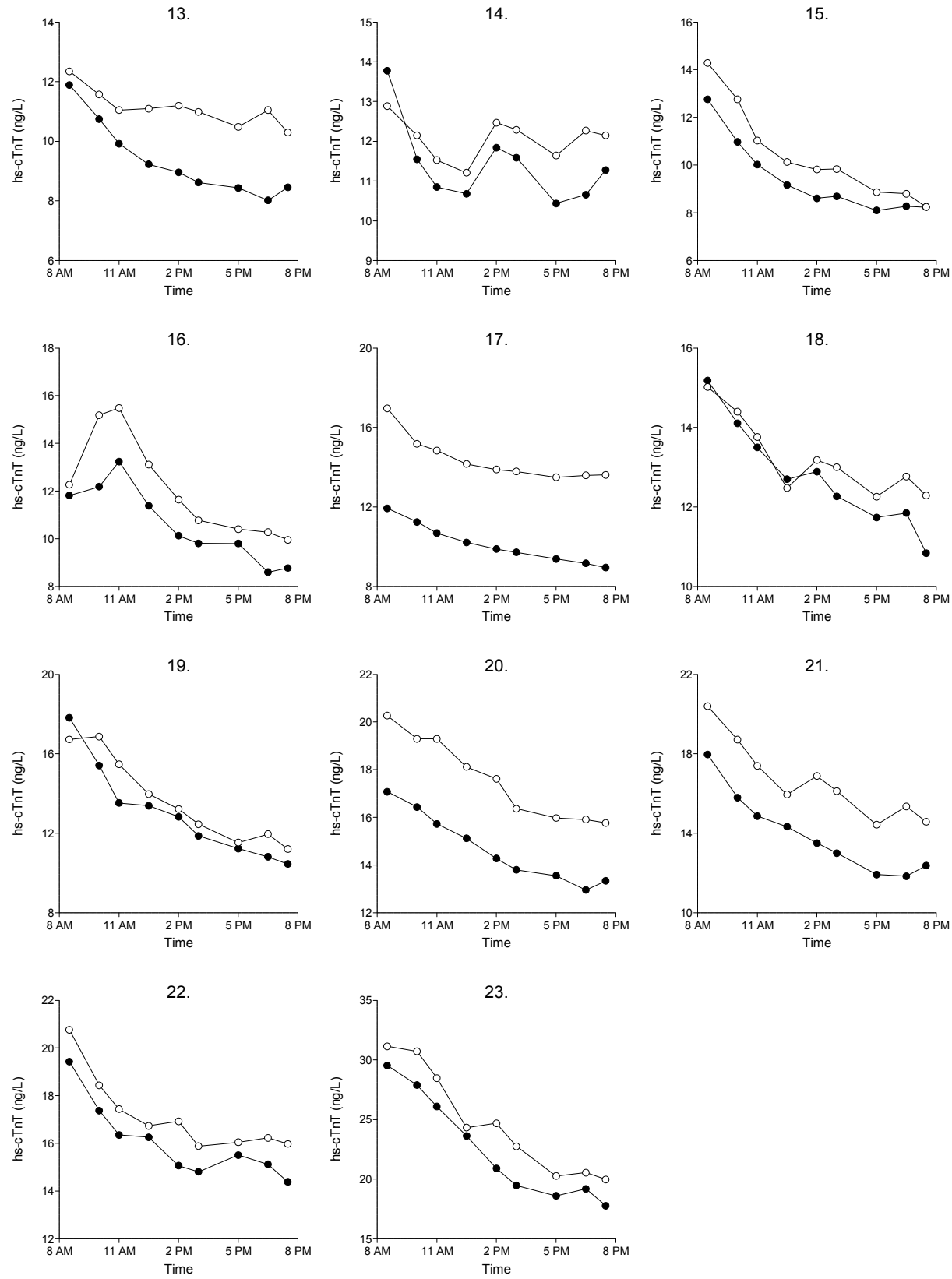

Supplemental figure 1. Individual hs-cTnT profiles during the day (continued from previous page).

hs-cTnT levels were measured under sedentary (black circle) and habitual physical activity (white circle) conditions. Depicted are the mean of duplicate measurements. The numbering is identical to figure 2. 
A

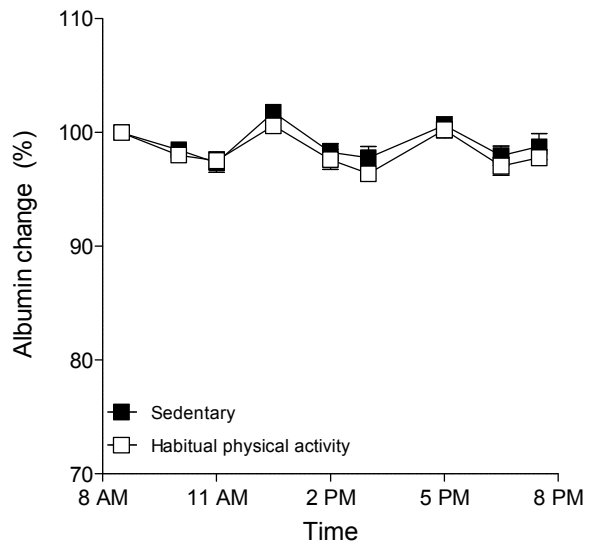

B

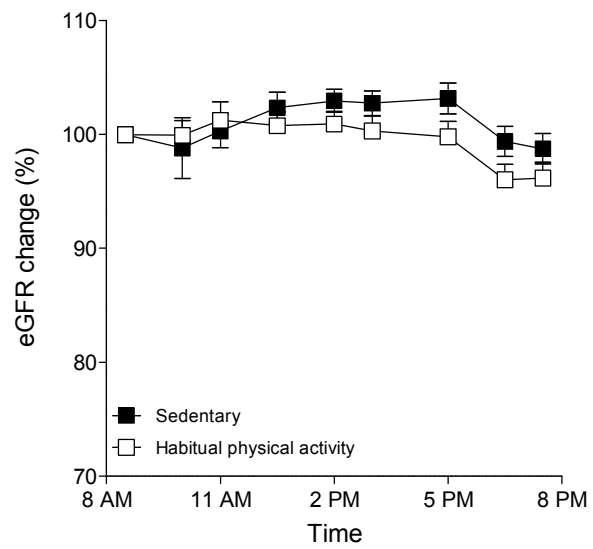

Supplemental figure 2. Percentage change of albumin and eGFR from baseline during the day under sedentary and habitual physical activity conditions.

Depicted are the mean \pm SEM of $\mathbf{A}$. albumin and $\mathbf{B}$. eGFR. 


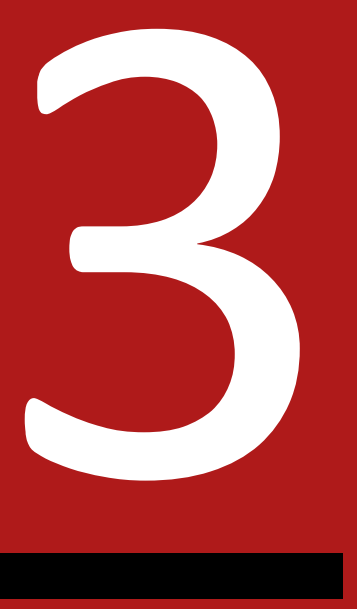




\section{A diurnal rhythm of cardiac troponin: consequences for the diagnosis of acute myocardial infarction}

Lieke Klinkenberg*, Karin Wildi*, Noreen van der Linden, Imre Kouw, Marijke Niens, Raphael Twerenbold, Maria Rubini Gimenez, Christian Puelacher, Jean Daniel Neuhaus, Petra Hillinger, Thomas Nestelberger, Jasper Boeddinghaus, Karin Grimm, Jeroen van Suijlen, Frans Tan, Joop ten Kate, Otto Bekers, Luc van Loon, Marja van Dieijen-Visser, Christian Mueller, Steven Meex

Submitted

*Equal contribution 


\section{Abstract}

Background Interpretation of serial high-sensitivity cardiac troponin (hs-cTn) measurements for the diagnosis of acute myocardial infarction (AMI) assumes random fluctuation of hs-cTn around an individual's homeostatic set-point.

Methods Two studies were conducted to challenge this diagnostic concept: Study 1 examined the presence of a diurnal hs-cTn rhythm by hourly blood sampling, day and night, in 24 individuals without a recent history of AMI. Study 2 assessed morning versus evening diagnostic accuracy of hs-cTnT and hs-cTnl in a prospective multicenter diagnostic study of 2782 unselected patients, presenting to the emergency department with acute chest pain. The final diagnosis was adjudicated by two independent cardiologists.

Results In study 1, hs-cTnT, but not hs-cTnl, exhibited a diurnal rhythm, characterized by gradually decreasing concentrations throughout daytime, rising concentrations during nighttime, to peak concentrations in the morning (mean $16.2 \mathrm{ng} / \mathrm{L}$ at 8:30 AM and $12.1 \mathrm{ng} / \mathrm{L}$ at 7:30 PM). In study 2, the hs-cTnT rhythm was confirmed by significantly higher hs-cTnT levels in early-morning presenters compared to evening presenters with an adjudicated diagnosis of non-cardiac disease. The diagnostic accuracy (area under the receiver-operating characteristic curve $[A \cup C]$ ) of hs-cTnT at presentation, $1 \mathrm{~h}$, and for the combination of absolute changes with presenting concentration, were very high and comparable among patients presenting early-morning as compared to evening (all $A U C>0.93$ ). hs-cTnl exhibited no diurnal rhythm with no differences in AUC among early-morning and evening presenters.

Conclusion Rhythmic diurnal variation of hs-cTnT is a general phenomenon that is not seen with hs-cTnl. While the diurnal hs-cTnT rhythm does not seem to affect the diagnostic accuracy of hs-cTnT for AMI, it should be considered when using hs-cTnT for screening purposes. 


\section{Introduction}

The early diagnosis of acute myocardial infarction (AMI) is mandatory for the timely initiation of evidence-based therapy and based on three key diagnostic tools: clinical assessment, the ECG, and cardiac troponins (cTn), quantitative markers of cardiomyocyte injury. ${ }^{1}$ Troponin assays target one of two proteins - cardiac troponin I (cTnl) or cardiac troponin $\mathrm{T}$ (cTnT) - with distinct biochemical properties, but comparable diagnostic performance. ${ }^{2}$ The advent of high-sensitivity (hs) cTn assays for both cTnl and CTnT has expedited the early diagnosis of $\mathrm{AMI}^{3-5}$ but also resulted in the detection of previously unnoticed cardiomyocyte injury in multiple other conditions such as diabetes, chronic kidney disease, frailty, or even apparently healthy elderly individuals. ${ }^{6-10}$ Key to accurately discriminate between acute and chronic causes of cardiomyocyte injury is serial testing and the assessment of kinetic changes in hs-cTn as recommended in the current guidelines. ${ }^{11-14}$ A critical assumption when interpreting changes between serial measurements is that blood concentrations of hs-cTn fluctuate randomly around a homeostatic set-point. This dogma of random fluctuation was recently challenged by the intriguing observation that hs-cTnT exhibited a diurnal rhythm in a small group of male subjects with type 2 diabetes. ${ }^{15}$ The diurnal hs-cTnT rhythm was characterized by peak-levels during morning hours, a gradual decrease during the day, and a rising pattern at nighttime. ${ }^{15}$

The generalizability of the diurnal rhythm of hs-cTnT beyond male type 2 diabetes patients and whether hs-cTnl and hs-cTnT are equally affected is unknown. It is also unknown, whether - if confirmed - diurnal rhythmic oscillation in blood concentrations of hs-cTn affect the diagnostic accuracy of hs-cTn for AMI. This possibility is substantiated by the observation that the magnitude of physiological change over the day, exceeded clinically applied delta-change criteria for AMI in some individuals. ${ }^{1,15}$

The present study was conducted to quantify the rhythmic diurnal oscillation of hscTnl and hs-cTnT in currently asymptomatic women and men. Next, we analyzed data from a multicenter diagnostic study, enrolling patients presenting to the emergency department with suspected AMI, to determine whether rhythmic diurnal oscillation and thereby the time of presentation affects the diagnostic accuracy of hs-cTnl and hs-cTnT.

\section{Methods}

Two studies were conducted: 1. a standardized biological variation study with hourly blood sampling day and night, to ascertain and quantify circadian rhythms of hs-cTnT and hs-cTnl (January 2013-May 2014); 2. a prospective diagnostic study (APACE; Advan- 
tageous Predictors of Acute Coronary syndrome Evaluation). APACE is an ongoing prospective international multicenter study aiming to advance the early diagnosis of AMI. ${ }^{2,4,16-18}$ Both studies complied with the Declaration of Helsinki and were approved by the local institutional review board and ethics committee at each institution. All participants provided written informed consent.

\section{Study 1: Diurnal rhythm under standardized conditions}

The study population consisted of 24 individuals; 19 males and 5 females (mean age 72 \pm 7 years, supplemental table 1). Exclusion criteria were: a history of AMI or stroke less than 12 months prior to inclusion. The diurnal hs-cTnT variation of 7 male subjects has been described before as part of a biological variation study of hs-cTnT in patients with type 2 diabetes (supplemental figure $1 A$ and 2, participants $4,5,7,11,16,20,24) .{ }^{15}$ To extend these pilot findings to both sexes and individuals with normal glucose homeostasis, 17 additional subjects (12 men, 5 women) were included in the present study. Participants were asked to refrain from exhaustive physical activities and exercise training 2 days before the test period. After an overnight fast, subjects arrived at the laboratory by car or public transportation. From 8:30 AM till 9:30 AM the next day, subjects were restricted to the laboratory environment and sampled hourly $(8 \mathrm{~mL})$. At night (11:30 PM till 7:00 AM), extension lines were used to minimize disturbance of participants' sleep. Meals were consumed at 8:30 AM, 12:30 PM and 5:30 PM (breakfast, lunch and dinner, respectively). Blood samples were collected in both serum (sensitive cTnl [s-cTnl] Beckman Coulter) and EDTA tubes (hs-cTnT Roche and hs-cTnl Abbott) and measured in duplicate. Cosinor rhythmometry was used to fit diurnal hs-cTn variation to a cosine curve and quantify the magnitude of hs-cTn oscillation.

\section{Study 2: Diurnal rhythm and diagnostic accuracy for myocardial infarction}

To investigate the clinical effects of rhythmic diurnal oscillations of hs-cTn, we evaluated whether the time of presentation to the emergency department affected the diagnostic accuracy of hs-cTn for AMI in the APACE study. The study population consisted of 2782 consecutive patients older than 18 years presenting to the emergency department between April 2006 and June 2013 with symptoms suggestive of AMI with onset or peak of symptoms within the last $12 \mathrm{~h}$ (supplemental table 2). Patients with renal failure requiring dialysis were excluded from the study.

For this analysis patients were excluded if $A$. the adjudicated diagnosis was STelevation AMI $(n=108)$ as biomarkers in general do not have a role in the rapid diagnosis of ST-elevation AMI, B. the final diagnosis remained unclear after adjudication and at least one cTn level was elevated (possibly indicating presence of AMI) ( $n=92)$, or C. the exact presentation time was not recorded $(n=48)$. 
Adjudication of the final diagnosis is extensively described in the supplemental methods. Briefly, two independent cardiologists adjudicated the final diagnosis by reviewing all available medical records - patient history, physical examination, results of laboratory testing (including levels of cTn), radiologic testing, ECG, echocardiography, cardiac exercise test, lesion severity and morphology in coronary angiography - pertaining to the patient from the time of emergency department presentation to 90 day follow up. In situations of disagreement about the diagnosis, cases were reviewed and adjudicated in conjunction with a third cardiologist.

After hospital discharge, patients were contacted after 3, 12 and 24 months by telephone calls or in written form. Information regarding death was furthermore obtained from the national registry on mortality, the hospitals' diagnosis registry, and the family physicians' records.

Cosinor rhythmometry results from study 1 were used to divide the day in two timeframes: a 'hs-cTn high level phase' (early-morning) and a 'hs-cTn low level phase' (evening). Diagnostic accuracy of hs-cTn at presentation, at $1 \mathrm{~h}$, and the combination of absolute changes with baseline concentration were the primary diagnostic end points, as they reflect the most important diagnostic variables in clinical practice. ${ }^{1,12,13}$

\section{Laboratory analysis}

CTnT was measured by using the high-sensitivity CTnT assay of Roche Diagnostics; the $99^{\text {th }}$ percentile among healthy subjects is $14 \mathrm{ng} / \mathrm{L}$, with a $10 \%$ analytical variation at 13 ng/L. ${ }^{19}$ Abbott high-sensitivity CTnl was measured with the STAT high-sensitivity troponin I assay. According to the manufacturer, the $99^{\text {th }}$ percentile is $26.2 \mathrm{ng} / \mathrm{L}$ with a corresponding coefficient of variation of $<5 \%$. The high-sensitivity cTnl assay of Siemens has a $99^{\text {th }}$ percentile concentration of $9 \mathrm{ng} / \mathrm{L}$ and a coefficient of variation of $10 \%$ at 3 $\mathrm{ng} / \mathrm{L}$ (according to the manufacturer). s-cTnl (Beckman Coulter) was measured by using the Access AccuTnl+3 Assay; with a $99^{\text {th }}$ percentile limit of $40 \mathrm{ng} / \mathrm{L}$ with a corresponding $10 \%$ imprecision, as specified by the manufacturer.

\section{Statistical analysis}

Diurnal troponin changes were analyzed by fitting the data to a cosine curve by using the method of cosinor-rhythmometry. ${ }^{15,20}$ Evidence of a diurnal rhythm was indicated by a significant cosinor model fit. Receiver-operating characteristics (ROC) curves were constructed to assess the sensitivity and specificity of hs-cTn for the diagnosis of AMI in early-morning presenters and evening presenters. The comparison of independent areas under the ROC curves (AUC) was performed as recommend by Hanley and McNeil. ${ }^{21,22}$ Similarly, AUCs were compared in early-morning presenters and evening presenters for the prognostic accuracy of hs-cTn at presentation to predict all-cause mortality during follow-up. 
All hypothesis testing was 2-tailed and a p-value of less than 0.05 was considered statistically significant. Analyses were performed using SPSS version 22 (IBM SPSS Statistics) and MedCalc 10.3.4.

\section{Results}

hs-cTnT exhibits a diurnal rhythm

To investigate the diurnal rhythm of hs-cTn, blood samples were collected hourly over a time span of 25h (8:30 AM - 9:30 AM the next day, supplemental figure 1A). In all participants, we found rhythmic circadian hs-cTnT (Roche) variation, characterized by gradually decreasing concentrations throughout daytime (mean $16.2 \mathrm{ng} / \mathrm{L}$ at 8:30 AM and mean $12.1 \mathrm{ng} / \mathrm{L}$ at 7:30 PM) and rising concentrations during nighttime, to peak concentrations in the morning (mean $16.2 \mathrm{ng} / \mathrm{L}$ at 8:30 AM the next day, figure $1 \mathrm{~A}$, supplemental figure 2). Subjects with the highest hs-cTnT concentrations displayed the largest amplitude of the diurnal oscillation, illustrated by the highly significant correlation between hs-cTnT level at 8:30 AM and the range of individual changes during the $25 \mathrm{~h}$ period (Pearson's $R^{2} 0.70, p<0.001$, supplemental figure 3 ).

A

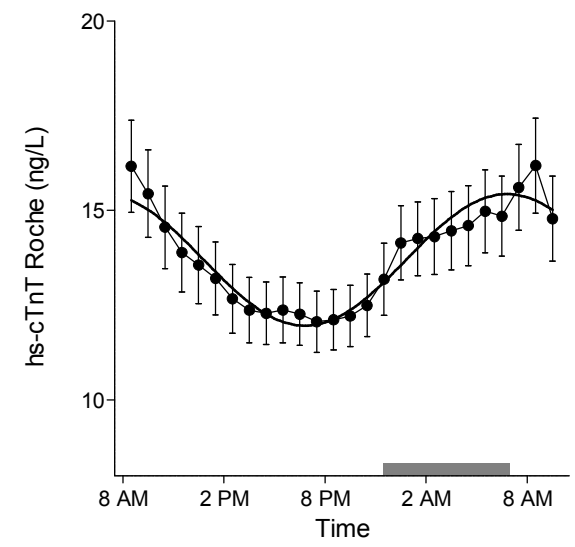

B

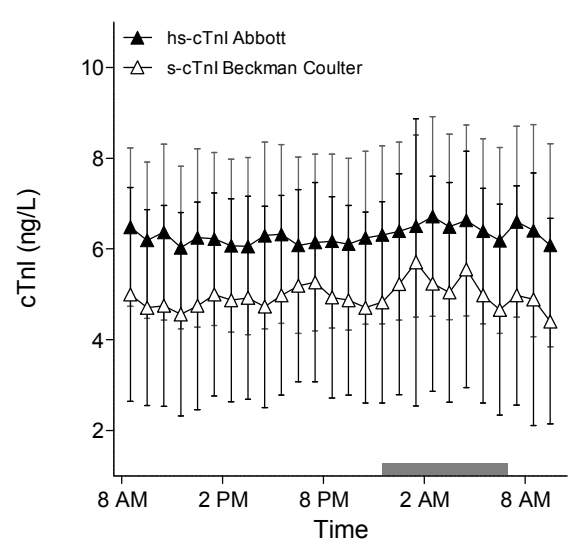

Figure 1. Diurnal variation of hs-cTnT, but not hs-cTnl, is a general phenomenon.

A. Mean \pm SEM $25 \mathrm{~h}$ hs-cTnT profile and fitted cosine curve. The cosine curve is described as $Z(t)=13.70+1.7$. $\cos (15 t-337.19)$, with a $R^{2}$ goodness of fit of $0.92(p<0.001)$. The time of peak was calculated at 6:58 AM. B. Mean \pm SEM 25h (h)s-cTnl profile. Subjects slept in a supine position between 11:30 PM and 7:00 AM (gray area). Error bars are black (s-cTnl Beckman Coulter) and gray (hs-cTnl Abbott). 
Based on the rhythmic variation, we tested the hs-cTnT data for a periodic signal using single cosinor analysis under the assumption of a $24 \mathrm{~h}$ period. In all but one individual (participant 1), the observed pattern correlated significantly to the fitted cosine model, as described by the mesor, amplitude and acrophase ( $R^{2}$ range: $0.44-0.91$, all $p$ $\leq 0.001$, table 1). Correction for possible posture-induced changes in plasma volume did not abrogate the diurnal rhythm (supplemental figure 4). ${ }^{23}$

Table 1. The individual diurnal variation of hs-cTnT is significantly described by a cosine curve. ${ }^{a}$

\begin{tabular}{|c|c|c|c|c|c|}
\hline Participant $^{\mathrm{b}}$ & $\begin{array}{l}\text { Mesor, } \\
\mathrm{ng} / \mathrm{L}\end{array}$ & $\begin{array}{l}\text { Amplitude, } \\
\mathrm{ng} / \mathrm{L}\end{array}$ & $\begin{array}{l}\text { Acrophase, } \\
\text { h }\end{array}$ & $\mathrm{R}^{2}$ & $p$-value \\
\hline 1 & 5.6 & 0.2 & $10: 45$ AM & 0.23 & 0.05 \\
\hline 2 & 7.3 & 1.2 & $5: 39$ AM & 0.82 & $<0.001 *$ \\
\hline 3 & 9.4 & 2.4 & $5: 47 \mathrm{AM}$ & 0.89 & $<0.001 *$ \\
\hline 4 & 8.4 & 1.7 & $7: 16$ AM & 0.66 & $<0.001^{*}$ \\
\hline 5 & 8.6 & 0.9 & $6: 29$ AM & 0.65 & $<0.001 *$ \\
\hline 6 & 8.9 & 0.9 & 5:01 AM & 0.70 & $<0.001 *$ \\
\hline 7 & 10.0 & 1.0 & $7: 35 \mathrm{AM}$ & 0.55 & $<0.001 *$ \\
\hline 8 & 10.6 & 0.9 & 5:57 AM & 0.54 & $<0.001 *$ \\
\hline 9 & 10.5 & 0.9 & 5:12 AM & 0.68 & $<0.001^{*}$ \\
\hline 10 & 12.7 & 0.7 & $2: 56 \mathrm{AM}$ & 0.44 & $0.001 *$ \\
\hline 11 & 12.9 & 2.4 & 6:03 AM & 0.81 & $<0.001^{*}$ \\
\hline 12 & 12.8 & 1.3 & $5: 18 \mathrm{AM}$ & 0.64 & $<0.001 *$ \\
\hline 13 & 15.6 & 3.8 & $6: 03 \mathrm{AM}$ & 0.81 & $<0.001 *$ \\
\hline 14 & 13.8 & 2.2 & 8:17 AM & 0.86 & $<0.001^{*}$ \\
\hline 15 & 15.3 & 2.7 & 8:53 AM & 0.77 & $<0.001 *$ \\
\hline 16 & 17.5 & 4.0 & $6: 37 \mathrm{AM}$ & 0.91 & $<0.001 *$ \\
\hline 17 & 16.3 & 1.9 & $10: 26 \mathrm{AM}$ & 0.57 & $<0.001 *$ \\
\hline 18 & 16.1 & 1.4 & $8: 26 \mathrm{AM}$ & 0.79 & $<0.001 *$ \\
\hline 19 & 19.7 & 3.7 & 5:29 AM & 0.90 & $<0.001 *$ \\
\hline 20 & 19.5 & 4.1 & 9:08 AM & 0.80 & $<0.001^{*}$ \\
\hline 21 & 19.4 & 2.2 & 4:35 AM & 0.86 & $<0.001 *$ \\
\hline 22 & 17.8 & 1.9 & $5: 29$ AM & 0.79 & $<0.001^{*}$ \\
\hline 23 & 18.3 & 2.4 & 8:19 AM & 0.82 & $<0.001^{*}$ \\
\hline 24 & 21.7 & 2.3 & 9:18 AM & 0.74 & $<0.001 *$ \\
\hline
\end{tabular}

${ }^{a} Z(t)=M+A \cdot \cos (\omega t+\phi)+e(t)$, where $Z(t)$ and $e(t)$ represent the measured concentration and the error between the cosinor model and the measurement, respectively, $\mathrm{M}$ the mesor (value about which oscillation occurs), A the amplitude (distance from mesor to peak), $\omega$ the angular frequency (degrees per unit time, with $360^{\circ}$ representing a complete cycle), $\phi$ the acrophase (timing of peak in degrees). ${ }^{b}$ Subjects were ranked on the basis of their visual range of hs-cTnT concentration over the day. The numbering used in table 1 is extended to supplemental figure 1, 2, 5 and 6 . *Significant after Bonferroni correction, $p=0.0021(0.05 / 24)$. 
hs-cTnl levels fluctuate at random

Distinct from hs-cTnT, hs-cTnl (Abbott) and s-cTnl (Beckman Coulter) levels fluctuated randomly around the subject's homeostatic set-point. Mean hs-cTnl and s-cTnl concentrations were $6.5 \mathrm{ng} / \mathrm{L}$ and $5.0 \mathrm{ng} / \mathrm{L}$ at 8:30 AM and $6.1 \mathrm{ng} / \mathrm{L}$ and 5.3 ng/L at 7:30 PM, respectively (figure $1 B$ and $1 C$, supplemental figure 5 and 6 ). Accordingly, in all but one participant, the $25 \mathrm{~h}$ hs-cTnl and s-cTnl profile did not fit significantly to a cosine curve. Even participant 24, with hs-cTnl and s-cTnl values above the $99^{\text {th }}$ percentile upper reference limit, and with the most prominent diurnal oscillations for hs-cTnT, showed relatively stable hs-cTnl and s-cTnl levels during the $25 \mathrm{~h}$ test period (supplemental figure 5 and 6). One individual had a hs-cTnl day-curve that could be fitted to a cosinus model (supplemental figure 5, participant 7). This curve, however, was characterized by an acrophase that was almost 10h out of phase compared to the mean hs-cTnT acrophase (10:30 PM vs. mean hs-cTnT acrophase of 6:58 AM).

\section{Diurnal rhythm of hs-cTn in chest pain patients}

To investigate whether rhythmic diurnal hs-cTn oscillation is also evident in a clinical setting, we compared hs-cTnT (Roche) and hs-cTnl (Abbott and Siemens) concentrations from 2782 patients included in the APACE trial. The adjudicated final diagnosis was AMI in $17.5 \%$, unstable angina in $9.8 \%$, cardiac non-coronary disease in $14.3 \%$, non-cardiac chest pain in 53.3\%, and unknown (but not AMI) in 5.1\%. APACE patients were classified in one of two timeframes that were derived from the observed diurnal rhythm of hscTnT under standardized conditions (figure 1A): a 'hs-cTnT high-level phase' in the earlymorning (11:00 PM till 2:00 PM) and a 'hs-cTnT low-level phase' in the evening (2:00 PM till 11:00 PM). hs-cTnT also showed a diurnal rhythm in patients with adjudicated diagnoses of non-cardiac disease and other cardiac disorders with significantly higher hscTnT levels in early-morning presenters compared to evening presenters. These differences were statistically significant, but small in absolute terms, and were not seen in patients with AMI or unstable angina (table 2).

The diurnal hs-cTnT rhythm overall did not affect diagnostic accuracy for AMI. Diagnostic accuracy of hs-cTnT as quantified by the area under the receiver-operating characteristics curve (AUC) at presentation, at $1 \mathrm{~h}$, and the combination of absolute changes with baseline value were very high and comparable among patients presenting in the early-morning and in the evening (all AUC >0.93; figure 2, table 3). In contrast, AUC of absolute changes within the first hour were lower in the early-morning (AUC 0.90 [95\% Cl: $0.89-0.92$ ]) as compared to the evening (AUC 0.95 [95\% Cl: $0.93-0.97], p$ $=0.01)$. 
Table 2. Early-morning and evening levels of hs-cTnT and hs-cTnl according to final diagnosis.

\begin{tabular}{|c|c|c|c|c|}
\hline Timepoint & Adjudicated diagnosis & $\begin{array}{l}\text { Early-morning, } \\
\text { median (IQR) }\end{array}$ & $\begin{array}{l}\text { Evening, }{ }^{\mathrm{b}} \\
\text { median (IQR) }\end{array}$ & $p$-value ${ }^{c}$ \\
\hline \multicolumn{5}{|c|}{ hs-cTnT Roche, ng/L } \\
\hline \multirow[t]{5}{*}{ Oh } & AMI & $60(28-153)$ & $60(25-119)$ & 0.94 \\
\hline & Unstable angina & $11(7-16)$ & $12(6-16)$ & 0.73 \\
\hline & Cardiac cause not CAD & $14(7-30)$ & $10(6-26)$ & 0.04 \\
\hline & Non-cardiac cause & $6(4-10)$ & $5(3-8)$ & $<0.001$ \\
\hline & Unknown & $7(4-10)$ & $5(3-10)$ & 0.20 \\
\hline \multirow[t]{5}{*}{$1 \mathrm{~h}$} & AMI & $68(32-193)$ & $88(34-164)$ & 0.49 \\
\hline & Unstable angina & $12(7-17)$ & $13(7-16)$ & 0.52 \\
\hline & Cardiac cause not CAD & $15(7-30)$ & $10(6-27)$ & 0.07 \\
\hline & Non-cardiac cause & $6(4-10)$ & $5(4-8)$ & 0.004 \\
\hline & Unknown & $7(4-10)$ & $6(4-10)$ & 0.34 \\
\hline \multicolumn{5}{|c|}{ hs-cTnl Siemens, ng/L } \\
\hline \multirow[t]{5}{*}{ Oh } & AMI & $199(27-1270)$ & $158(22-559)$ & 0.35 \\
\hline & Unstable angina & $6(3-14)$ & $7(4-17)$ & 0.55 \\
\hline & Cardiac cause not CAD & $9(3-50)$ & $6(2-24)$ & 0.45 \\
\hline & Non-cardiac cause & $2(1-5)$ & $2(1-5)$ & 0.09 \\
\hline & Unknown & $3(2-7)$ & $2(1-4)$ & 0.17 \\
\hline \multirow[t]{5}{*}{$1 \mathrm{~h}$} & AMI & $275(46-1357)$ & $203(55-974)$ & 0.58 \\
\hline & Unstable angina & $6(4-15)$ & $8(4-17)$ & 0.66 \\
\hline & Cardiac cause not CAD & $10(3-70)$ & $6(3-23)$ & 0.21 \\
\hline & Non-cardiac cause & $3(1-5)$ & $2(1-6)$ & 0.36 \\
\hline & Unknown & $4(2-7)$ & $3(1-4)$ & 0.22 \\
\hline \multicolumn{5}{|c|}{ hs-cTnl Abbott, ng/L } \\
\hline \multirow[t]{5}{*}{ Oh } & AMI & $114(22-628)$ & $107(21-424)$ & 0.82 \\
\hline & Unstable angina & $6(3-12)$ & $8(4-13)$ & 0.49 \\
\hline & Cardiac cause not CAD & $9(4-32)$ & $7(3-18)$ & 0.46 \\
\hline & Non-cardiac cause & $3(2-5)$ & $3(2-5)$ & 0.39 \\
\hline & Unknown & $3(3-5)$ & $3(2-4)$ & 0.44 \\
\hline \multirow[t]{5}{*}{$1 \mathrm{~h}$} & AMI & $151(28-871)$ & $217(38-613)$ & 0.52 \\
\hline & Unstable angina & $6(4-12)$ & $9(4-14)$ & 0.46 \\
\hline & Cardiac cause not CAD & $10(3-50)$ & $7(4-15)$ & 0.40 \\
\hline & Non-cardiac cause & $3(2-5)$ & $3(2-6)$ & 0.58 \\
\hline & Unknown & $3(2-5)$ & $3(2-3)$ & 0.85 \\
\hline
\end{tabular}

a Early-morning: 11:00 PM - 2:00 PM. ${ }^{b}$ Evening: 2:00 PM - 11:00 PM. ${ }^{\mathrm{c}}$ Mann Whitney U test. CAD; coronary artery disease. 
Early-morning

A

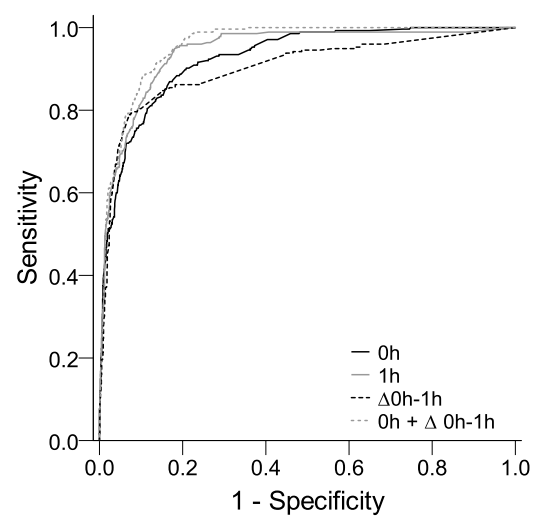

B

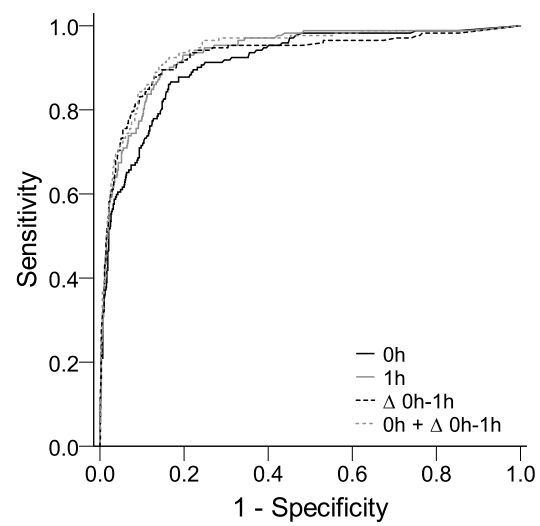

C

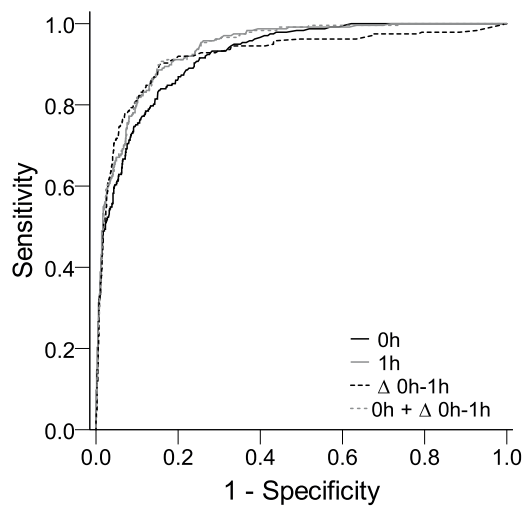

Evening
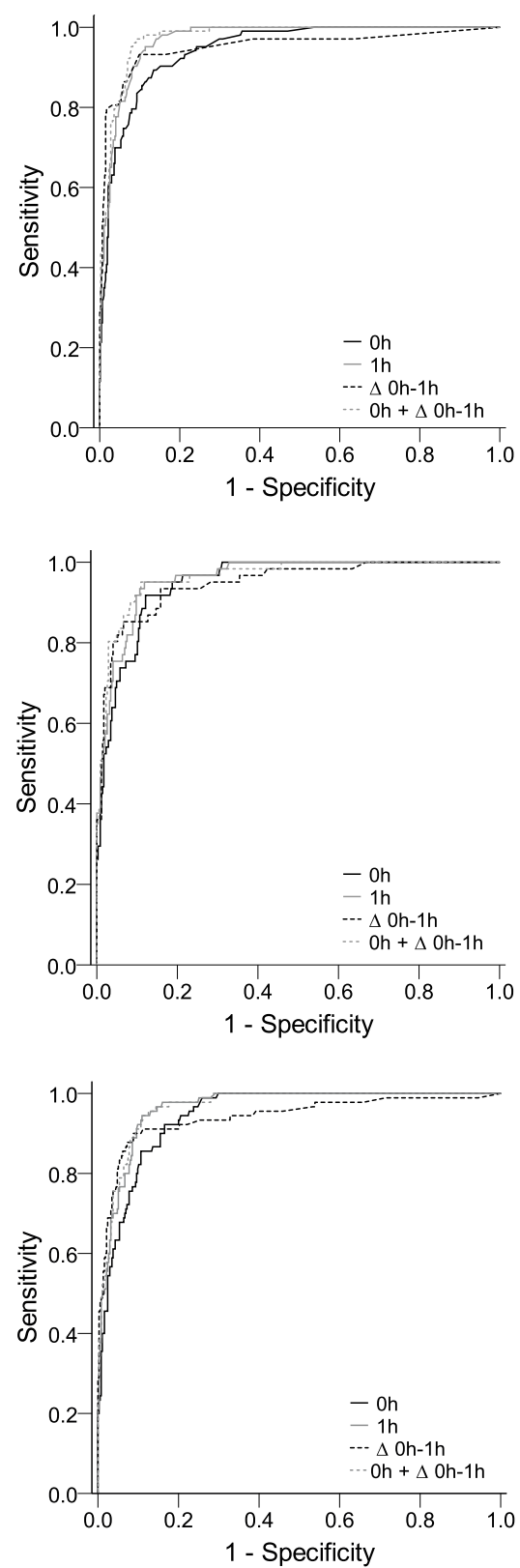

Figure 2. Diagnostic performance of hs-cTnT and hs-cTnl for AMl in early-morning and evening presenters.

Receiver-operating characteristic curves show the diagnostic accuracy for AMI of A. hs-cTnT Roche B. hs-cTnl Siemens and C. hs-cTnl Abbott at presentation, at 1h, absolute changes within the first hour, and the combination of presenting levels with absolute changes in early-morning (11:00 PM - 2:00 PM) and evening (2:00 PM - 11:00 PM) presenters. 
Table 3. Diagnostic accuracy of hs-cTnT and hs-cTnl changes in early-morning and evening presenters.

\begin{tabular}{|c|c|c|c|}
\hline Timepoint & $\begin{array}{l}\text { Early-morning, } \\
\text { AUC }(95 \% \mathrm{CI})\end{array}$ & $\begin{array}{l}\text { Evening, } \\
\text { AUC (95\% CI) }\end{array}$ & p-value \\
\hline \multicolumn{4}{|l|}{ hs-cTnT Roche } \\
\hline $\mathrm{Oh}$ & $0.93(0.92-0.94)$ & $0.94(0.92-0.96)$ & 0.58 \\
\hline $1 \mathrm{~h}$ & $0.94(0.93-0.95)$ & $0.97(0.95-0.98)$ & 0.10 \\
\hline$\Delta \mathrm{Oh}-1 \mathrm{~h}$ & $0.90(0.89-0.92)$ & $0.95(0.93-0.97)$ & 0.01 \\
\hline $0 \mathrm{~h}+\Delta \mathrm{Oh}-1 \mathrm{~h}$ & $0.96(0.95-0.97)$ & $0.98(0.96-0.99)$ & 0.20 \\
\hline \multicolumn{4}{|c|}{ hs-cTnl Siemens } \\
\hline $\mathrm{Oh}$ & $0.92(0.90-0.93)$ & $0.94(0.92-0.96)$ & 0.32 \\
\hline $1 \mathrm{~h}$ & $0.93(0.92-0.95)$ & $0.96(0.94-0.98)$ & 0.24 \\
\hline$\Delta 0 \mathrm{~h}-1 \mathrm{~h}$ & $0.93(0.91-0.94)$ & $0.95(0.92-0.97)$ & 0.39 \\
\hline $\mathrm{Oh}+\Delta \mathrm{Oh}-1 \mathrm{~h}$ & $0.94(0.92-0.95)$ & $0.96(0.94-0.98)$ & 0.26 \\
\hline \multicolumn{4}{|l|}{ hs-cTnl Abbott } \\
\hline $\mathrm{Oh}$ & $0.93(0.91-0.94)$ & $0.94(0.92-0.96)$ & 0.50 \\
\hline $1 \mathrm{~h}$ & $0.94(0.91-0.94)$ & $0.96(0.94-0.98)$ & 0.09 \\
\hline$\Delta 0 \mathrm{~h}-1 \mathrm{~h}$ & $0.92(0.91-0.94)$ & $0.94(0.92-0.96)$ & 0.35 \\
\hline $\mathrm{Oh}+\Delta \mathrm{Oh}-1 \mathrm{~h}$ & $0.94(0.92-0.95)$ & $0.97(0.95-0.98)$ & 0.11 \\
\hline
\end{tabular}

${ }^{\mathrm{a}}$ Early morning: 11:00 PM - 2:00 PM. ${ }^{\mathrm{b}}$ Evening: 2:00 PM - 11:00 PM.

$\mathrm{Cl}$; confidence interval.

In patients presenting very early ( $\leq 3 \mathrm{~h}$ ) after symptom onset $(n=1490,16.3 \%$ with $\mathrm{AMI}$ ), differences in AUC between early-morning presenters and patients presenting in the evening seemed to be more pronounced and were statistically significant or of borderline significance for the absolute level at $1 \mathrm{~h}$, the absolute change within the first hour after presentation, and the combination of the absolute level at presentation and the 1 h change (table 4). The prognostic accuracy of hs-cTnT at presentation for allcause mortality within one year, as quantified by the AUC was moderate-to-high and comparable among all adjudicated diagnoses between early-morning presenters and evening presenters (supplemental table 3).

hs-cTnl (Abbott and Siemens) did not exhibit a diurnal rhythm, levels were similar among all adjudicated diagnoses between early-morning presenters and evening presenters (table 2). In addition, diagnostic accuracy of hs-cTnl was very high and comparable among patients presenting in the early-morning and patients presenting in the evening (all $A \cup C>0.92$, figure 2, table 3 and 4). 
Table 4. Diagnostic accuracy of hs-cTnT and hs-cTnl changes in early-morning and evening presenters $(\leq 3 \mathrm{~h}$ since symptom onset).

\begin{tabular}{|c|c|c|c|}
\hline Timepoint & $\begin{array}{l}\text { Early-morning, } \\
\text { AUC }(95 \% \mathrm{CI})\end{array}$ & $\begin{array}{l}\text { Evening, } \\
\text { AUC }(95 \% \mathrm{Cl})\end{array}$ & $p$-value \\
\hline \multicolumn{4}{|c|}{ hs-cTnT Roche } \\
\hline Oh & $0.90(0.89-0.92)$ & $0.93(0.91-0.96)$ & 0.26 \\
\hline $1 \mathrm{~h}$ & $0.94(0.92-0.95)$ & $0.98(0.96-0.99)$ & 0.05 \\
\hline$\Delta 0 \mathrm{~h}-1 \mathrm{~h}$ & $0.90(0.88-0.92)$ & $0.95(0.93-0.97)$ & 0.01 \\
\hline$O h+\Delta O h-1 h$ & $0.95(0.93-0.96)$ & $0.98(0.96-0.99)$ & 0.06 \\
\hline \multicolumn{4}{|c|}{ hs-cTnl Siemens } \\
\hline Oh & $0.91(0.88-0.93)$ & $0.94(0.90-0.96)$ & 0.36 \\
\hline $1 \mathrm{~h}$ & $0.94(0.92-0.96)$ & $0.96(0.93-0.98)$ & 0.63 \\
\hline$\Delta 0 h-1 h$ & $0.93(0.91-0.94)$ & $0.95(0.92-0.97)$ & 0.41 \\
\hline $0 h+\Delta O h-1 h$ & $0.95(0.93-0.97)$ & $0.97(0.94-0.98)$ & 0.61 \\
\hline \multicolumn{4}{|l|}{ hs-cTnl Abbott } \\
\hline $\mathrm{Oh}$ & $0.92(0.90-0.94)$ & $0.94(0.91-0.97)$ & 0.42 \\
\hline $1 \mathrm{~h}$ & $0.94(0.92-0.96)$ & 0.97 (0.94 - 0.99) & 0.25 \\
\hline$\Delta 0 h-1 h$ & $0.91(0.90-0.94)$ & $0.94(0.92-0.96)$ & 0.20 \\
\hline$O h+\Delta O h-1 h$ & $0.94(0.92-0.96)$ & 0.97 (0.94 - 0.99) & 0.19 \\
\hline
\end{tabular}

a Early morning: 11:00 PM - 2:00 PM. ${ }^{b}$ Evening: 2:00 PM - 11:00 PM.

$\mathrm{Cl}$; confidence interval.

\section{Discussion}

The aim of the present study was to validate the diurnal troponin rhythm as a general phenomenon and to evaluate whether diurnal oscillation affect the diagnostic accuracy for AMI. We report seven major findings:

First, we identified rhythmic diurnal hs-cTnT variation as a general phenomenon, characterized by gradually declining levels during daytime followed by rising concentrations during nighttime to peak concentrations at again (early) morning hours. The rhythm is irrespective of sex and glucose metabolism status, with the largest amplitude of the oscillation seen in stable subjects with the highest hs-cTnT concentrations. Second, the diurnal rhythm is restricted to hs-cTnT, with no evidence for rhythmic variation detected in hs-cTnl and s-cTnl as quantified by three different cTnl assays. We can only speculate which physiological differences between cTnT and CTnl underlie the observed differences in the diurnal rhythmicity. Renal function may play a role, as various observations suggests that deteriorating renal function is stronger related to elevated cTnT levels than cTnl levels. ${ }^{8,24-27}$ However, it is unknown whether diurnal varia- 
tions in renal function would be sufficient to cause rather pronounced relative changes in hs-cTnT in the normal range. Accordingly, differences in cTn metabolism including repair mechanisms may also play a role. Third, these results could be reproduced in the large APACE study: significantly higher hs-cTnT levels were evident in early-morning presenters compared to evening presenters with an adjudicated final diagnosis of noncardiac disease. Fourth, diagnostic accuracy of hs-cTnT for AMI overall was very high in patients presenting in the early-morning as well as patients presenting in the evening and not significantly different between the two. Fifth, the physiological rise and fall of hs-cTnT during the day significantly affected the diagnostic accuracy of absolute changes within the first hour after presentation with lower AUC in the early-morning (when non-cardiac disease and healthy persons have higher levels) as compared to the evening (when non-cardiac disease and healthy persons have lower levels). As in current clinical practice absolute changes are not used as a primary diagnostic variable, but only secondary to the absolute level at presentation and at $1 \mathrm{~h}$, or in conjunction with the absolute level at presentation/1h, the clinical impact - if any - of the circadian rhythm in hs-cTnT seems to be very small overall. Sixth, in patients presenting very early $(\leq 3 \mathrm{~h})$ after chest pain onset, the difference in AUC seemed to be more pronounced. This observation can be well explained that in early presenters the true circadian baseline level has still a stronger impact on hs-cTnT blood concentrations in AMI, as the added hs-cTnT component resulting exclusively from the AMI event is much smaller very early versus later after chest pain onset. As the magnitude of the difference in AUC was small, the clinical relevance of this difference seems to be very small at most also in early presenters. The circadian rhythm in hs-cTnT may well however affect the diagnostic accuracy of hs-cTnT when used as a screening tool for cardiovascular disease, where much smaller differences exist between health and disease. Accordingly, screening with its need for serial testing over years should be scheduled on the same time of the day in the individual patient. Seventh, prognostic accuracy of hs-cTnT for all-cause mortality within one year was moderate-to-high and not affected by the circadian rhythm.

Our findings extend and corroborate recent pilot data suggesting a diurnal rhythm for hs-cTnT in a small group of male subjects with type 2 diabetes. ${ }^{15}$ As circulating concentrations of hs-cTn are defined by the extent of release and clearance, this study does not allow to quantify the influence of both processes on the diurnal hs-cTnT rhythm. Nevertheless, the rhythmicity of this cardiac biomarker is in line with the circadian organization of the cardiovascular system. ${ }^{28,29}$ Synchronizing physiological functions to the natural $24 \mathrm{~h}$ light-dark cycle, for example by upregulating body temperature and blood pressure before the time of awakening, anticipates to the demands of daytime activities. Circadian rhythmicity has in addition been described for many cardiovascular traits: heart rate, sympathetic activity, vascular resistance, the renin-angiotensinaldosteron system, prothrombotic tendency, platelet aggregability and the fibrinolytic 
system. $^{30-34}$ Identical to hs-cTnT concentrations, all these components exhibit peak activity in (early) morning hours. The physiological rhythmicity of these cardiovascular components is reflected in the increasing intensity of cardiovascular events in the morning hours. ${ }^{35}$ For example, the incidence of AMI, sudden cardiac death and stroke display a significant $24 \mathrm{~h}$ variation, characterized by a higher frequency in morning hours. ${ }^{36-38}$ Even the clock time of AMI onset is associated with clinical course and survival, with the largest infarct sizes and higher mortality found among patients with AMI that commence in morning hours. ${ }^{39,40}$

Potential limitations of our study merit consideration. First, study 2 was conducted with patients presenting to the emergency department with symptoms suggestive of AMI. Additional studies in patients presenting to a general practitioner, which in general have a much lower pretest-probability for $\mathrm{AMI}$, are required to learn whether the diurnal hs-cTnT rhythm affects the diagnostic accuracy in this setting. Second, this diagnostic study required written informed consent. Therefore, invariably the rate of enrollment was lower than in registries. Third, we cannot comment on diurnal rhythms in patients with terminal kidney failure requiring dialysis, since such patients were excluded from our study.

To conclude, rhythmic diurnal variation of hs-cTnT is a general phenomenon, that is not seen with hs-cTnl, and should trigger additional studies to better understand its pathophysiology. Fortunately, the rhythmic diurnal variation of hs-cTnT does not seem to affect the high diagnostic accuracy of hs-cTnT in the diagnosis of AMI. It may however affect its use as a screening tool, where much smaller differences exist between health and disease.

\section{Acknowledgements}

We gratefully acknowledge the laboratory assistance of Vincent Kleijnen, Joan Senden and Antoine Zorenc. Study 1 was supported by a research grant from Stichting De Weijerhorst to M.v.D.-V. Abbott Diagnostics and Beckman Coulter provided troponin I assays to M.v.D.-V. and S.M. for study 1 . Study 2 was supported by research grants from the Swiss National Science Foundation, the Swiss Heart Foundation, the Cardiovascular Research Foundation Basel, the University Hospital Basel, Abbott, Roche, Nanosphere, Siemens, 8sense, Bühlmann and BRAHMS. 


\section{References}

1. Amsterdam EA, Wenger NK, Brindis RG, et al. 2014 AHA/ACC Guideline for the Management of Patients With Non-ST-Elevation Acute Coronary Syndromes: A Report of the American College of Cardiology/American Heart Association Task Force on Practice Guidelines. J Am Coll Cardiol. 2014;64(24):e139-228.

2. Rubini Gimenez M, Twerenbold R, Reichlin T, et al. Direct comparison of high-sensitivity cardiac troponin I vs. T for the early diagnosis of acute myocardial infarction. Eur Heart J. 2014;35(34):2303-2311.

3. Keller T, Zeller T, Peetz D, et al. Sensitive troponin I assay in early diagnosis of acute myocardial infarction. N Engl J Med. 2009;361(9):868-877.

4. Reichlin T, Hochholzer W, Bassetti S, et al. Early diagnosis of myocardial infarction with sensitive cardiac troponin assays. N Engl J Med. 2009;361(9):858-867.

5. Mills NL, Churchhouse AM, Lee KK, et al. Implementation of a sensitive troponin I assay and risk of recurrent myocardial infarction and death in patients with suspected acute coronary syndrome. JAMA. 2011;305(12):1210-1216.

6. de Lemos JA. Increasingly sensitive assays for cardiac troponins: a review. JAMA. 2013;309(21):22622269.

7. Selvin E, Lazo M, Chen Y, et al. Diabetes, Pre-Diabetes and Incidence of Subclinical Myocardial Damage. Circulation. 2014;130(16):1374-1382.

8. deFilippi C, Seliger SL, Kelley W, et al. Interpreting cardiac troponin results from high-sensitivity assays in chronic kidney disease without acute coronary syndrome. Clin Chem. 2012;58(9):1342-1351.

9. van der Linden $\mathrm{N}$, Tieland $\mathrm{M}$, Klinkenberg $\mathrm{L}$, et al. The effect of a six-month resistance-type exercise training program on the course of high sensitive cardiac troponin $\mathrm{T}$ levels in (pre)frail elderly. Int J Cardiol. 2014;175(2):374-375.

10. Eggers KM, Venge $\mathrm{P}$, Lindahl B, Lind L. Cardiac troponin I levels measured with a high-sensitive assay increase over time and are strong predictors of mortality in an elderly population. J Am Coll Cardiol. 2013;61(18):1906-1913.

11. Giannitsis E, Katus HA. Cardiac troponin level elevations not related to acute coronary syndromes. Nat Rev Cardiol. 2013;10(11):623-634.

12. Thygesen K, Alpert JS, Jaffe AS, et al. Third universal definition of myocardial infarction. Eur Heart J. 2012;33(20):2551-2567.

13. Hamm CW, Bassand JP, Agewall S, et al. ESC Guidelines for the management of acute coronary syndromes in patients presenting without persistent ST-segment elevation: The Task Force for the management of acute coronary syndromes (ACS) in patients presenting without persistent ST-segment elevation of the European Society of Cardiology (ESC). Eur Heart J. 2011;32(23):2999-3054.

14. Thygesen K, Mair J, Giannitsis E, et al. How to use high-sensitivity cardiac troponins in acute cardiac care. Eur Heart J. 2012;33(18):2252-2257.

15. Klinkenberg LJ, van Dijk JW, Tan FE, van Loon LJ, van Dieijen-Visser MP, Meex SJ. Circulating cardiac troponin T exhibits a diurnal rhythm. J Am Coll Cardiol. 2014;63(17):1788-1795.

16. Haaf $P$, Reichlin $T$, Twerenbold $R$, et al. Risk stratification in patients with acute chest pain using three high-sensitivity cardiac troponin assays. Eur Heart J. 2014;35(6):365-375.

17. Reichlin $T$, Hochholzer W, Stelzig $C$, et al. Incremental value of copeptin for rapid rule out of acute myocardial infarction. J Am Coll Cardiol. 2009;54(1):60-68.

18. Reiter M, Twerenbold R, Reichlin $\mathrm{T}$, et al. Early diagnosis of acute myocardial infarction in patients with pre-existing coronary artery disease using more sensitive cardiac troponin assays. Eur Heart J. 2012;33(8):988-997.

19. Giannitsis E, Kurz K, Hallermayer K, Jarausch J, Jaffe AS, Katus HA. Analytical validation of a highsensitivity cardiac troponin T assay. Clin Chem. 2010;56(2):254-261.

20. Nelson W, Tong YL, Lee JK, Halberg F. Methods for cosinor-rhythmometry. Chronobiologia. 1979;6(4):305-323.

21. Hanley JA, McNeil BJ. The meaning and use of the area under a receiver operating characteristic (ROC) curve. Radiology. 1982;143(1):29-36.

22. Hanley JA, McNeil BJ. A method of comparing the areas under receiver operating characteristic curves derived from the same cases. Radiology. 1983;148(3):839-843. 
23. Dill DB, Costill DL. Calculation of percentage changes in volumes of blood, plasma, and red cells in dehydration. J Appl Physiol. 1974;37(2):247-248.

24. Collinson PO, Heung YM, Gaze D, et al. Influence of population selection on the 99th percentile reference value for cardiac troponin assays. Clin Chem. 2012;58(1):219-225.

25. Apple FS, Murakami MM, Pearce LA, Herzog CA. Predictive value of cardiac troponin I and $T$ for subsequent death in end-stage renal disease. Circulation. 2002;106(23):2941-2945.

26. Hickman PE, Koerbin G, Southcott E, et al. Newer cardiac troponin I assays have similar performance to troponin T in patients with end-stage renal disease. Ann Clin Biochem. 2007;44(Pt 3):285-289.

27. Jacobs LH, van de Kerkhof J, Mingels AM, et al. Haemodialysis patients longitudinally assessed by highly sensitive cardiac troponin T and commercial cardiac troponin T and cardiac troponin I assays. Ann Clin Biochem. 2009;46(Pt 4):283-290.

28. Martino TA, Sole MJ. Molecular time: an often overlooked dimension to cardiovascular disease. Circ Res. 2009;105(11):1047-1061.

29. Sato M, Matsuo T, Atmore H, Akashi M. Possible contribution of chronobiology to cardiovascular health. Front Physiol. 2013;4:409.

30. Mancia G, Ferrari A, Gregorini L, et al. Blood pressure and heart rate variabilities in normotensive and hypertensive human beings. Circ Res. 1983;53(1):96-104.

31. Williams GH, Cain JP, Dluhy RG, Underwood RH. Studies of the control of plasma aldosterone concentration in normal man. I. Response to posture, acute and chronic volume depletion, and sodium loading. J Clin Invest. 1972;51(7):1731-1742.

32. Tofler GH, Brezinski D, Schafer Al, et al. Concurrent morning increase in platelet aggregability and the risk of myocardial infarction and sudden cardiac death. N Engl J Med. 1987;316(24):1514-1518.

33. Fearnley GR, Balmforth G, Fearnley E. Evidence of a diurnal fibrinolytic rhythm; with a simple method of measuring natural fibrinolysis. Clin Sci (Lond). 1957;16(4):645-650.

34. Panza JA, Epstein SE, Quyyumi AA. Circadian variation in vascular tone and its relation to alphasympathetic vasoconstrictor activity. N Engl J Med. 1991;325(14):986-990.

35. Manfredini R, Boari B, Salmi R, et al. Twenty-four-hour patterns in occurrence and pathophysiology of acute cardiovascular events and ischemic heart disease. Chronobiol Int. 2013;30(1-2):6-16.

36. Muller JE, Stone PH, Turi ZG, et al. Circadian variation in the frequency of onset of acute myocardial infarction. N Engl J Med. 1985;313(21):1315-1322.

37. Muller JE, Ludmer PL, Willich SN, et al. Circadian variation in the frequency of sudden cardiac death. Circulation. 1987;75(1):131-138.

38. Argentino C, Toni D, Rasura M, et al. Circadian variation in the frequency of ischemic stroke. Stroke. 1990;21(3):387-389.

39. Reiter R, Swingen C, Moore L, Henry TD, Traverse JH. Circadian dependence of infarct size and left ventricular function after ST elevation myocardial infarction. Circ Res. 2012;110(1):105-110.

40. Manfredini R, Boari B, Bressan S, et al. Influence of circadian rhythm on mortality after myocardial infarction: data from a prospective cohort of emergency calls. Am J Emerg Med. 2004;22(7):555-559.

41. International Diabetes Federation Guideline Development G. Global guideline for type 2 diabetes. Diabetes Res Clin Pract. 2014;104(1):1-52.

42. Inker $\mathrm{LA}$, Schmid $\mathrm{CH}$, Tighiouart $\mathrm{H}$, et al. Estimating glomerular filtration rate from serum creatinine and cystatin C. N Engl J Med. 2012;367(1):20-29. 


\section{Supplemental information}

\section{Supplemental methods}

Adjudication of the final diagnosis was performed centrally in a core lab (University Hospital Basel) and included two sets of cardiac troponin (cTn) measurements. ${ }^{2,4,16-18}$ Those performed clinically in each hospital at presentation and at 3 - 6 h and possibly also at 12 - $24 \mathrm{~h}$ according to current guidelines, ${ }^{1,13}$ as well as levels of high-sensitivity cardiac troponin $\mathrm{T}$ (hs-cTnT) measured from the study blood samples taken at presentation, at 1, 2, 3 and $6 \mathrm{~h}$ (if feasible) in order to take advantage of the higher sensitivity and higher overall diagnostic accuracy offered by hs-cTnT (this allows the additional detection of small acute myocardial infarctions [AMIs]). 3,4,16

AMI was defined and cTn levels interpreted as recommended in current guidelines. ${ }^{1,12,13}$ In brief, AMI was diagnosed when there was evidence of myocardial necrosis in association with a clinical setting consistent with myocardial ischemia. Myocardial necrosis was diagnosed by at least one cTn value above the $99^{\text {th }}$ percentile together with a significant rising and/or falling. ${ }^{1,12,13}$ All other patients were classified as 'No nonST elevation acute myocardial infarction (NSTEMI)' for this analysis, including in this group the categories of unstable angina (UA), non-cardiac chest pain (NCCP), cardiac but non-coronary disease (e.g. tachyarrhythmia, perimyocarditis), and symptoms of unknown origin with normal levels of cTn and hs-cTnT.

The following conventional cTn assays were used: For the Roche CTnT $4^{\text {th }}$ generation assay, the $10 \% \mathrm{CV}$ level is $0.035 \mu \mathrm{g} / \mathrm{L}$. The laboratories of the participating sites reported only two decimals; therefore $0.04 \mu \mathrm{g} / \mathrm{L}$ was used as a cut-off for myocardial necrosis. In order to fulfil the criteria of a significant change $\left(30 \%\right.$ of $99^{\text {th }}$ percentile or $10 \%$ CV level), a patient would e.g. need to have a level of $<0.01 \mu \mathrm{g} / \mathrm{L}$ at presentation and $0.04 \mu \mathrm{g} / \mathrm{L}$ at $6 \mathrm{~h}$. A patient would also qualify if the first level is $0.02 \mu \mathrm{g} / \mathrm{L}$ and the second $0.04 \mu \mathrm{g} / \mathrm{L}$. A patient would not fulfil the criteria if the first level is $0.03 \mu \mathrm{g} / \mathrm{L}$ and the second is $0.04 \mu \mathrm{g} / \mathrm{L}$. If the first level is $0.04 \mu \mathrm{g} / \mathrm{L}$, the second level needs to be at least $0.06 \mu \mathrm{g} / \mathrm{L}$. For the Abbott Axsym cTnl ADV, the $10 \% \mathrm{CV}$ level is $0.16 \mu \mathrm{g} / \mathrm{L}$. A patient having $0.16 \mu \mathrm{g} / \mathrm{L}$ at presentation would meet the criteria for significant change if the second was $\geq 0.21 \mu \mathrm{g} / \mathrm{L}$. A patient having $<0.12 \mu \mathrm{g} / \mathrm{L}$ at presentation (limit of detection) would qualify if the second is $>0.16 \mu \mathrm{g} / \mathrm{L}$. For the Beckmann Coulter Accu cTnl, the $10 \%$ $\mathrm{CV}$ level is $0.06 \mu \mathrm{g} / \mathrm{L}$. A patient having $0.06 \mu \mathrm{g} / \mathrm{L}$ at presentation would qualify if the second is $\geq 0.08 \mu \mathrm{g} / \mathrm{L}$. A patient having $0.05 \mu \mathrm{g} / \mathrm{L}$ at presentation would qualify if the second is $0.07 \mu \mathrm{g} / \mathrm{L}$, but not $0.06 \mu \mathrm{g} / \mathrm{L}$. A patient having undetectable $\mathrm{cTnl}$ (cTnl $<0.01$ $\mu \mathrm{g} / \mathrm{L}$ ) at presentation would qualify if the second is $\geq 0.06 \mu \mathrm{g} / \mathrm{L}$.

For hs-cTnT, the $99^{\text {th }}$ percentile $(14 \mathrm{ng} / \mathrm{L})$ was used as cut-off for myocardial necrosis. Absolute changes in hs-cTnT were used to determine significant changes based on 
the diagnostic superiority of absolute over relative changes. Based on studies of the biological variation of cTn as well as on data from previous chest pain cohort studies, a significant absolute change was defined as a rise or fall of at least $10 \mathrm{ng} / \mathrm{L}$ within $6 \mathrm{~h}$. In patients, in whom a $6 \mathrm{~h}$ hs-cTnT level was not available, changes were assessed at earlier time points. In an assumption of linearity, an absolute change of $6 \mathrm{ng} / \mathrm{L}$ within $3 \mathrm{~h}$ was considered.

\section{Supplemental tables}

Supplemental table 1. Study 1: Participants' characteristics.

\begin{tabular}{|c|c|}
\hline Characteristic & $\begin{array}{l}\text { All } \\
n=24\end{array}$ \\
\hline Age, years & $72 \pm 7$ \\
\hline Male gender & $19(79 \%)$ \\
\hline Type 2 Diabetes $^{a}$ & $7(29 \%)$ \\
\hline $\mathrm{BMI}, \mathrm{kg} / \mathrm{m}^{2}$ & $27 \pm 5$ \\
\hline Systolic blood pressure, ${ }^{\mathrm{b}} \mathrm{mm} \mathrm{Hg}$ & $140 \pm 15$ \\
\hline Diastolic blood pressure, ${ }^{b} \mathrm{~mm} \mathrm{Hg}$ & $68 \pm 8$ \\
\hline Fasting glucose, ${ }^{b} \mathrm{mmol} / \mathrm{L}$ & $5.9(5.2-6.7)$ \\
\hline $\mathrm{HbA} 1 \mathrm{c}, \mathrm{b} \%$ & $5.7(5.3-6.5)$ \\
\hline Total cholesterol, ${ }^{\mathrm{b}} \mathrm{mmol} / \mathrm{L}$ & $3.7(4.8-5.4)$ \\
\hline HDL cholesterol, ${ }^{\mathrm{b}} \mathrm{mmol} / \mathrm{L}$ & $1.3(1.6-1.9)$ \\
\hline LDL cholesterol, ${ }^{b} \mathrm{mmol} / \mathrm{L}$ & $1.7(2.5-3.0)$ \\
\hline Triglycerides, ${ }^{\mathrm{b}} \mathrm{mmol} / \mathrm{L}$ & $1.2(0.8-1.8)$ \\
\hline NTproBNP, ${ }^{b} \mathrm{pmol} / \mathrm{L}$ & $10.9(4.9-38.8)$ \\
\hline hs-cTnT Roche, ${ }^{b}$ ng/L & $16.1(10.7-21.1)$ \\
\hline hs-cTnl Abbott, ${ }^{b}$ ng/L & $4.6(3.0-7.0)$ \\
\hline s-cTnl Beckman Coulter, ${ }^{b}$ ng/L & $3.0(1.0-4.5)$ \\
\hline $\mathrm{eGFR},{ }^{\mathrm{bc}} \mathrm{mL} / \mathrm{min} / 1.73 \mathrm{~m}^{2}$ & $75(61-92)$ \\
\hline Oral blood glucose-lowering medication ${ }^{d}$ & $7(29 \%)$ \\
\hline Lipid-lowering medication $^{d}$ & $12(50 \%)$ \\
\hline Anti-hypertensive medication $^{d}$ & $10(42 \%)$ \\
\hline
\end{tabular}

Data is presented as mean $\pm \mathrm{SD}$, median (IQR) or $\mathrm{n}(\%)$.

${ }^{\mathrm{a}}$ Diabetes was defined as fasting plasma glucose $\geq 7.0 \mathrm{mmol} / \mathrm{L}$ and/or $\mathrm{HbA} 1 \mathrm{c} \geq 6.5 \% .{ }^{41}$ b Value at 8:30 AM day 1. ${ }^{c}$ eGFR, estimated glomerular filtration rate according to CKD-EPI Creatinine-Cystatin C equation. ${ }^{42 \mathrm{~d}}$ Medication status was stable for at least three months prior to inclusion, and continued as normal during the study period. 
Supplemental table 2. Study 2: Baseline characteristics of patients enrolled in the APACE trial.

\begin{tabular}{|c|c|c|c|c|}
\hline Characteristic & $\begin{array}{l}\text { All } \\
n=2782\end{array}$ & $\begin{array}{l}\text { AMI } \\
n=488\end{array}$ & $\begin{array}{l}\text { no AMI } \\
n=2294\end{array}$ & $p$-value ${ }^{a}$ \\
\hline Age, years & $61(49-74)$ & $72(59-80)$ & $59(47-72)$ & $<0.001$ \\
\hline Male gender & $1903(68.4 \%)$ & $360(73.8 \%)$ & $1543(67.3 \%)$ & 0.014 \\
\hline \multicolumn{5}{|l|}{ Risk factors } \\
\hline Hypertension & $1712(61.5 \%)$ & $381(78.1 \%)$ & $1331(58.0 \%)$ & $<0.001$ \\
\hline Hypercholesterolemia & $1390(50.0 \%)$ & $332(68.0 \%)$ & $1058(46.1 \%)$ & $<0.001$ \\
\hline Diabetes mellitus & $477(17.1 \%)$ & $126(25.8 \%)$ & $351(15.3 \%)$ & $<0.001$ \\
\hline Current or previous smoking & $1722(61.9 \%)$ & $317(65.0 \%)$ & 1405 (61.2\%) & 0.11 \\
\hline Family history & $767(27.6 \%)$ & $158(32.4 \%)$ & $609(26.5 \%)$ & 0.008 \\
\hline \multicolumn{5}{|l|}{ History } \\
\hline Coronary artery disease & $965(34.7 \%)$ & $242(49.6 \%)$ & $723(31.5 \%)$ & $<0.001$ \\
\hline Previous AMI & $662(23.8 \%)$ & $172(35.2 \%)$ & $490(21.4 \%)$ & $<0.001$ \\
\hline Previous revascularization & 775 (27.9\%) & $183(37.5 \%)$ & $592(25.8 \%)$ & $<0.001$ \\
\hline Peripheral artery disease & $169(6.1 \%)$ & $63(12.9 \%)$ & $106(4.6 \%)$ & $<0.001$ \\
\hline Previous stroke & $152(5.5 \%)$ & $44(9.0 \%)$ & $108(4.7 \%)$ & $<0.001$ \\
\hline \multicolumn{5}{|l|}{ ECG findings } \\
\hline Left bundle branch block & $79(2.8 \%)$ & $28(5.7 \%)$ & $51(2.2 \%)$ & $<0.001$ \\
\hline ST-segment elevation & $57(2.0 \%)$ & $13(2.7 \%)$ & $44(1.9 \%)$ & 0.28 \\
\hline ST-segment depression & $282(10.1 \%)$ & $146(29.9 \%)$ & $136(5.9 \%)$ & $<0.001$ \\
\hline T-wave inversion & $359(12.9 \%)$ & $120(24.6 \%)$ & $239(10.4 \%)$ & $<0.001$ \\
\hline $\mathrm{BMI}, \mathrm{kg} / \mathrm{m}^{2}$ & $26(24-30)$ & $26(24-29)$ & $26(24-30)$ & 0.66 \\
\hline eGFR, mL/min/1.73 $\mathrm{m}^{2}$ & $85(69-101)$ & $73(56-94)$ & $86(71-102)$ & $<0.001$ \\
\hline \multicolumn{5}{|l|}{ Medication at presentation } \\
\hline ASA/thienopyridine & 1111 (39.9\%) & $268(54.9 \%)$ & $843(36.7 \%)$ & $<0.001$ \\
\hline Beta-blockers & $966(34.7 \%)$ & $214(43.9 \%)$ & $752(32.8 \%)$ & $<0.001$ \\
\hline ACEIs/ARBs & $1069(38.4 \%)$ & $253(51.8 \%)$ & $816(35.6 \%)$ & $<0.001$ \\
\hline Calcium antagonists & $411(14.8 \%)$ & $100(20.5 \%)$ & $311(13.6 \%)$ & $<0.001$ \\
\hline
\end{tabular}

Data is presented as median (IQR) or $\mathrm{n}(\%)$.

${ }^{a}$ Comparisons between $\mathrm{AMI}$ and no AMI patients.

ACEIs; angiotensin-converting-enzyme inhibitors, ARBs; angiotensin II receptor blockers, ASA; acetylsalicylic acid. 
Supplemental table 3. Prognostic accuracy of hs-cTnT in early-morning and evening presenters for the prediction of all-cause mortality within one year.

\begin{tabular}{llll}
\hline Timepoint & $\begin{array}{l}\text { Early-morning, } \\
\text { AUC }(95 \% \mathrm{Cl})\end{array}$ & $\begin{array}{l}\text { Evening, } \\
\text { AUC }(95 \% \mathrm{Cl})\end{array}$ & p-value \\
\hline All patients & $0.74(0.71-0.77)$ & $0.82(0.81-0.84)$ & 0.22 \\
AMI & $0.57(0.48-0.66)$ & $0.66(0.37-0.62)$ & 0.65 \\
Unstable angina & $0.49(0.37-0.62)$ & $0.74(0.67-0.80)$ & 0.28 \\
Cardiac cause not CAD & $0.81(0.72-0.88)$ & $0.79(0.74-0.84)$ & 0.90 \\
Non-cardiac cause & $0.78(0.73-0.82)$ & $0.85(0.83-0.87)$ & 0.53 \\
Unknown & Insufficient data & Insufficient data & - \\
\hline
\end{tabular}

Early morning: 11:00 PM - 2:00 PM. ' Evening: 2:00 PM - 11:00 PM.

CAD; coronary artery disease, $\mathrm{Cl}$; confidence interval.

\section{Supplemental figures}

A

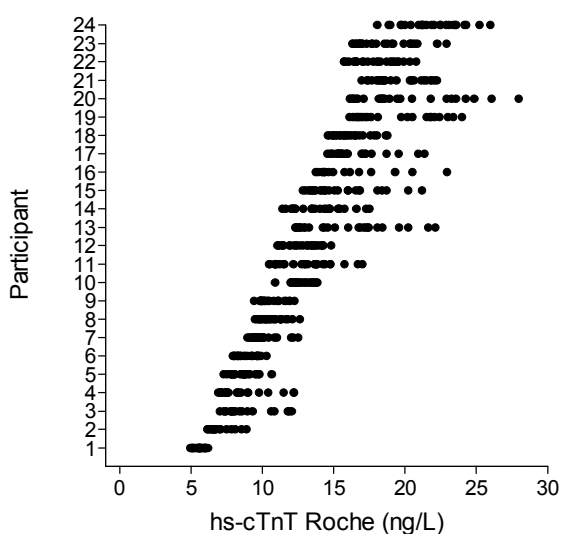

C

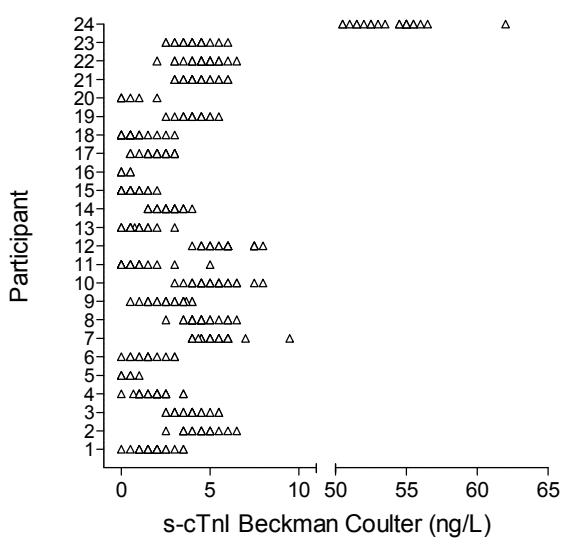

B

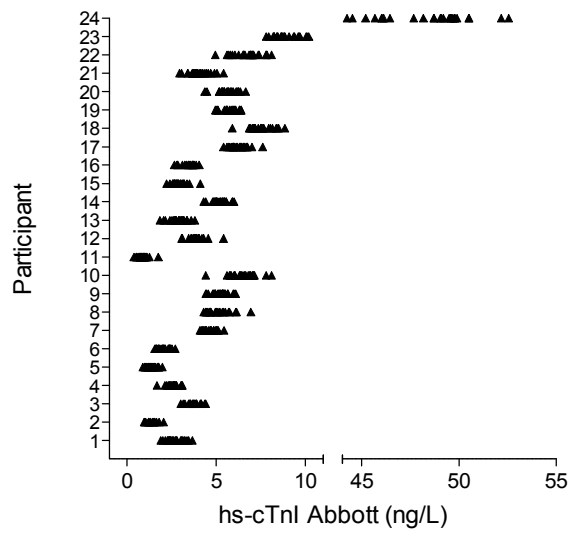

Supplemental figure 1. $25 \mathrm{~h}$ distribution of hscTnT, hs-cTnl and s-cTnl concentrations.

Distribution of A. hs-cTnT Roche B. hs-cTnl Abbott and C. s-cTnl Beckman Coulter in 24 subjects (8:30 AM - 9:30 AM the next day). Circles represent mean troponin values. Participants 1, 2, 8, 12 and 21 are female subjects. Subjects were ranked on the basis of their visual range of hs-cTnT concentration: generally, high subject number correspond to a wider range in hs-cTnT concentration. The ranking used for supplemental figure $1 \mathrm{~A}$ is extended to supplemental figure $1 \mathrm{~B}, 1 \mathrm{C}$, table 1 and supplemental figure 2, 5 and 6 . 

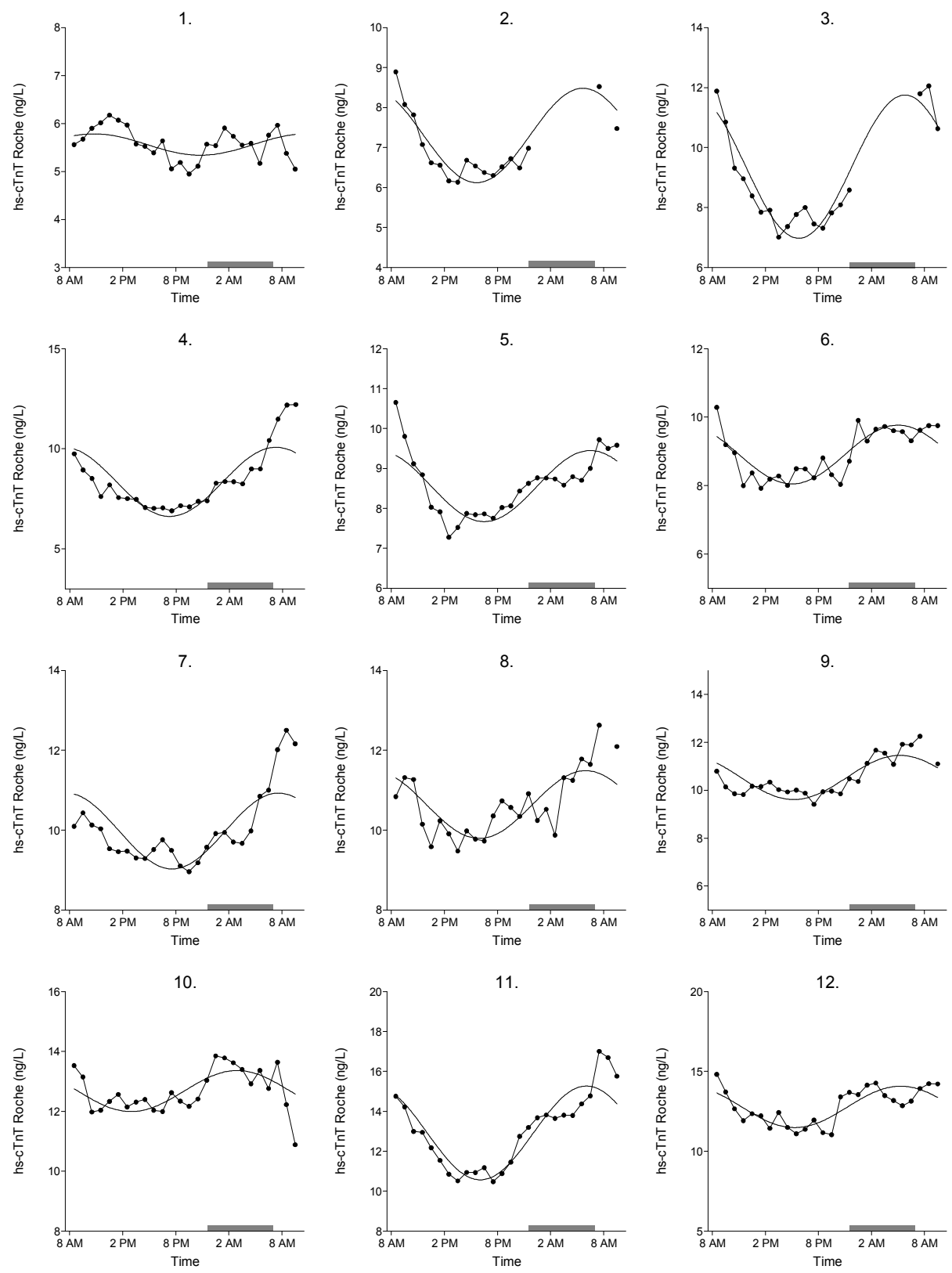

Supplemental figure 2. Diurnal hs-cTnT profiles and fitted cosine curves (continued on next page).

Depicted are the mean of the duplicate measurements. The numbering is identical to table 1 and supplemental figure 1, 5 and 6. Subjects slept in a supine position between 11:30 PM and 7:00 AM (gray area). Sampling in subjects 2, 3 and 16 was hindered throughout the night due to technical problems with the intravenous cannula. 

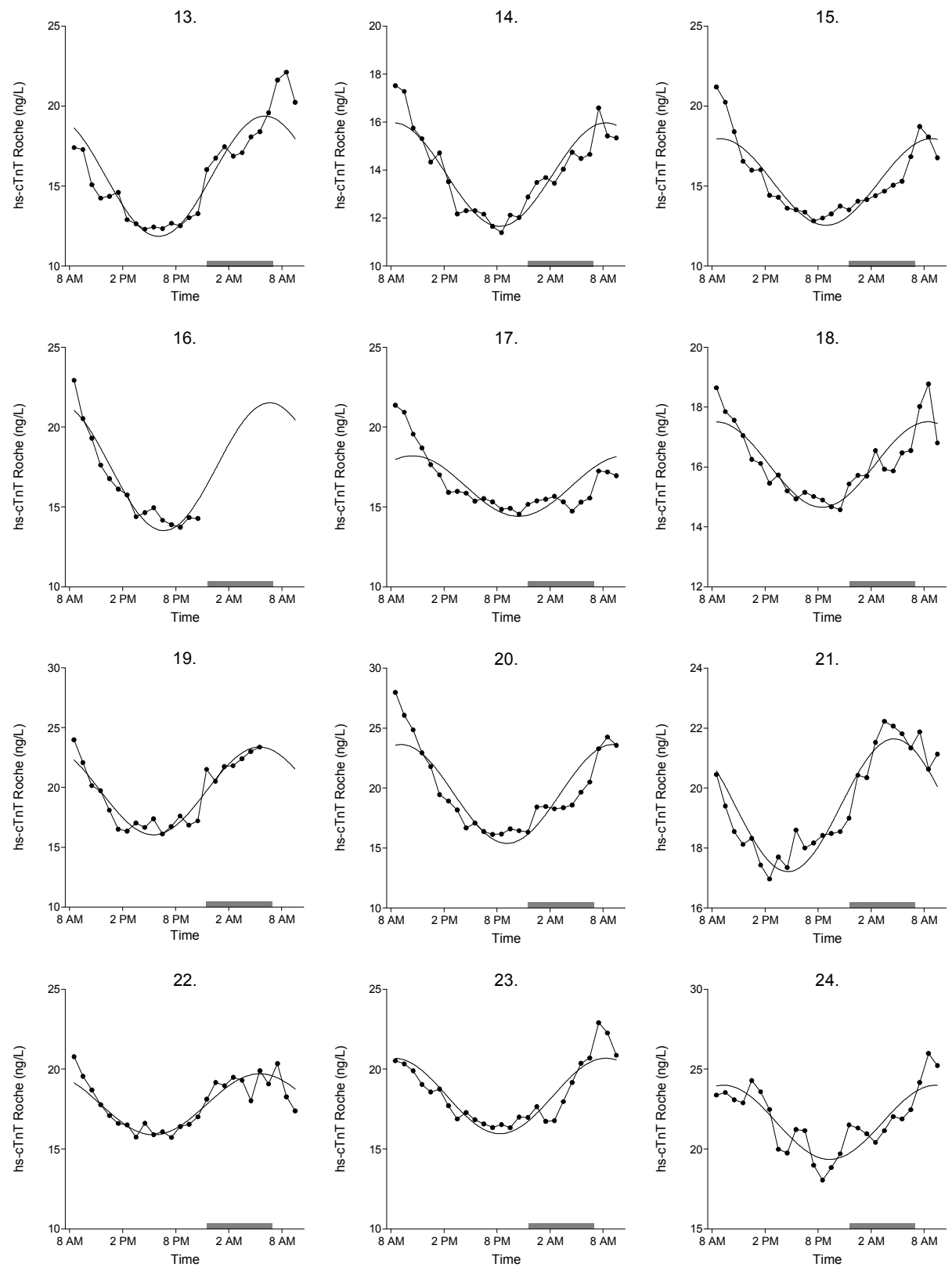

Supplemental figure 2. Diurnal hs-cTnT profiles and fitted cosine curves (continued from previous page).

Depicted are the mean of the duplicate measurements. The numbering is identical to table 1 and supplemental figure 1, 5 and 6. Subjects slept in a supine position between 11:30 PM and 7:00 AM (gray area). Sampling in subjects 2, 3 and 16 was hindered throughout the night due to technical problems with the intravenous cannula. 
Supplemental figure 3. hs-cTnT fluctuations are most prominent in individuals with the highest hs-cTnT concentrations.

Correlation between the hs-cTnT value at 8:30 AM day 1 and the maximal intra-individual changes during the $25 \mathrm{~h}$ test period (Pearson's $R^{2}$ 0.70, $\left.p<0.001\right)$.

Supplemental figure 4. Mean diurnal hs-cTnT profile corrected for possible plasma volume changes and fitted cosine curve.

Depicted are the mean \pm SEM $25 \mathrm{~h}$ hs-cTnT profile corrected for hemoglobin and hematocrit and fitted cosine curve. Subjects slept in a supine position between 11:30 PM and 7:00 AM (gray area). The cosine curve is described as $Z(t)$ $=14.75+2.34 \cdot \cos (15 t-305.56)$, with a $R^{2}$ goodness of fit of $0.94(p<0.001)$.
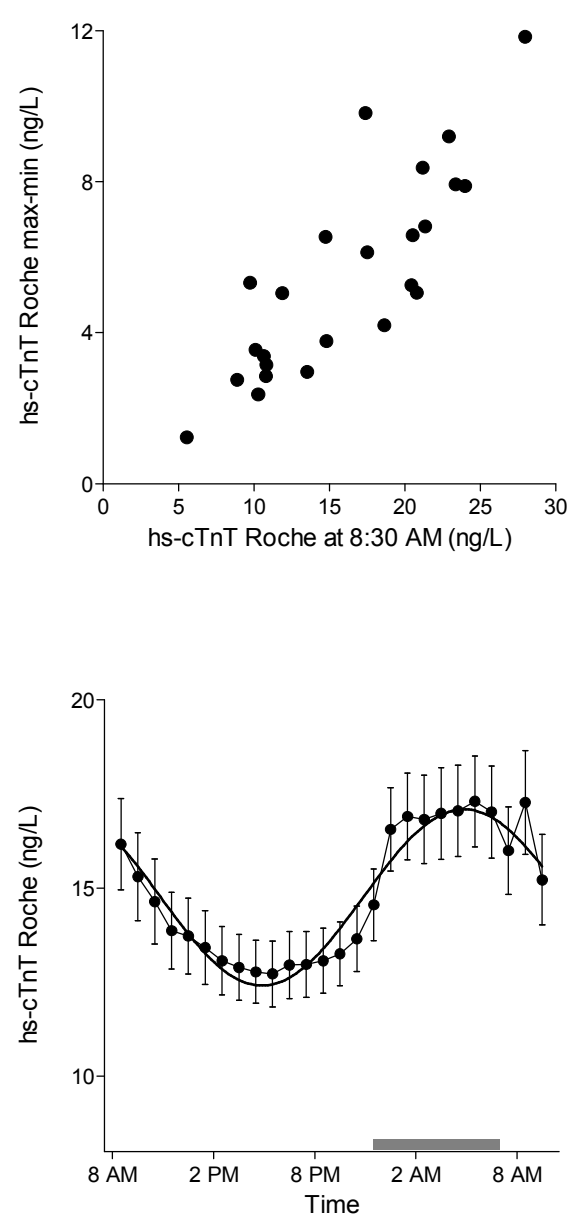

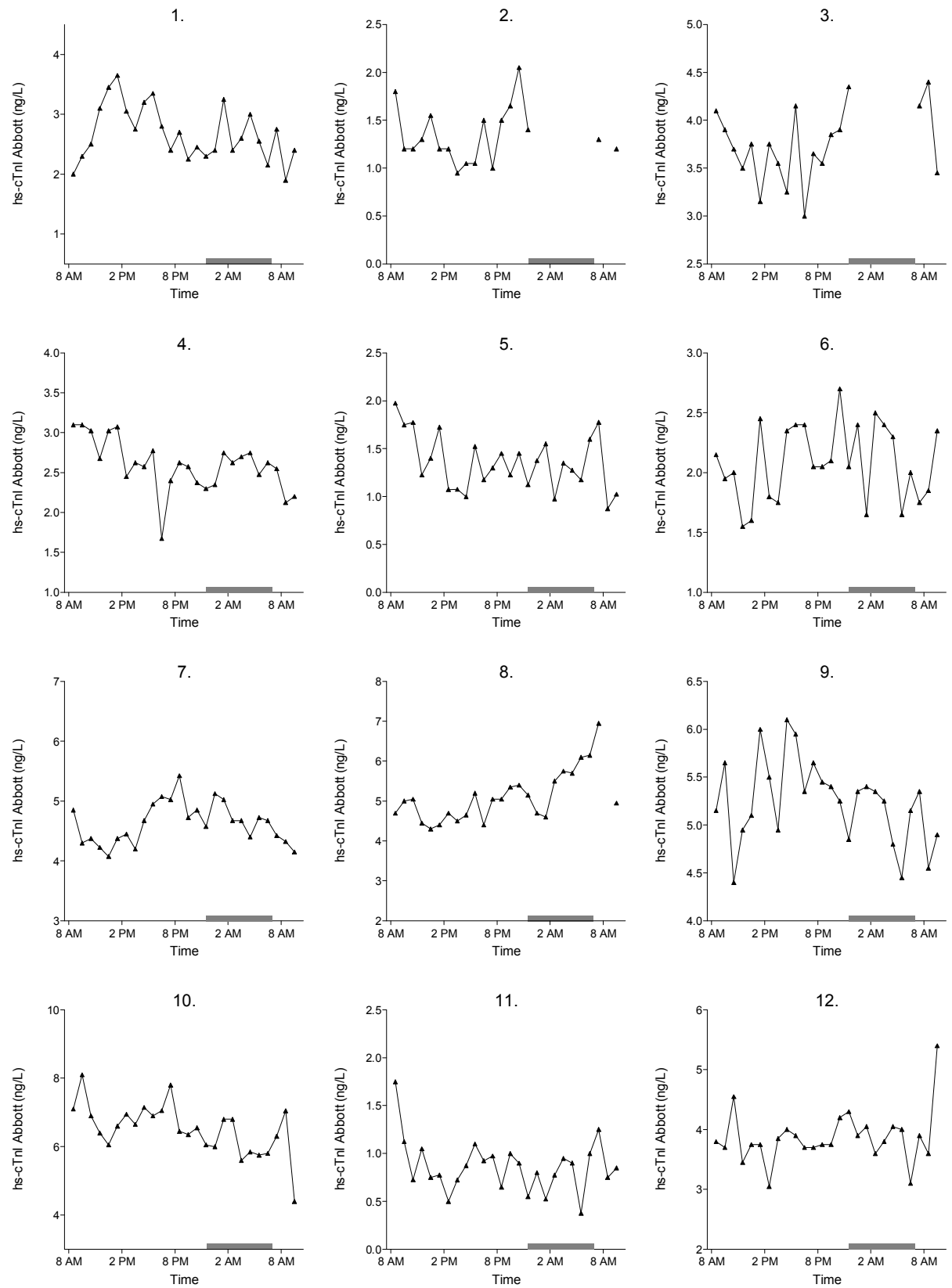

Supplemental figure 5. Diurnal hs-cTnl profiles (continued on next page).

Depicted are the mean of the duplicate measurements. The numbering is identical to table 1 and supplemental figure 1, 2 and 6. Subjects slept in a supine position between 11:30 PM and 7:00 AM (gray area). Sampling in subjects 2, 3 and 16 was hindered throughout the night due to technical problems with the intravenous cannula. 

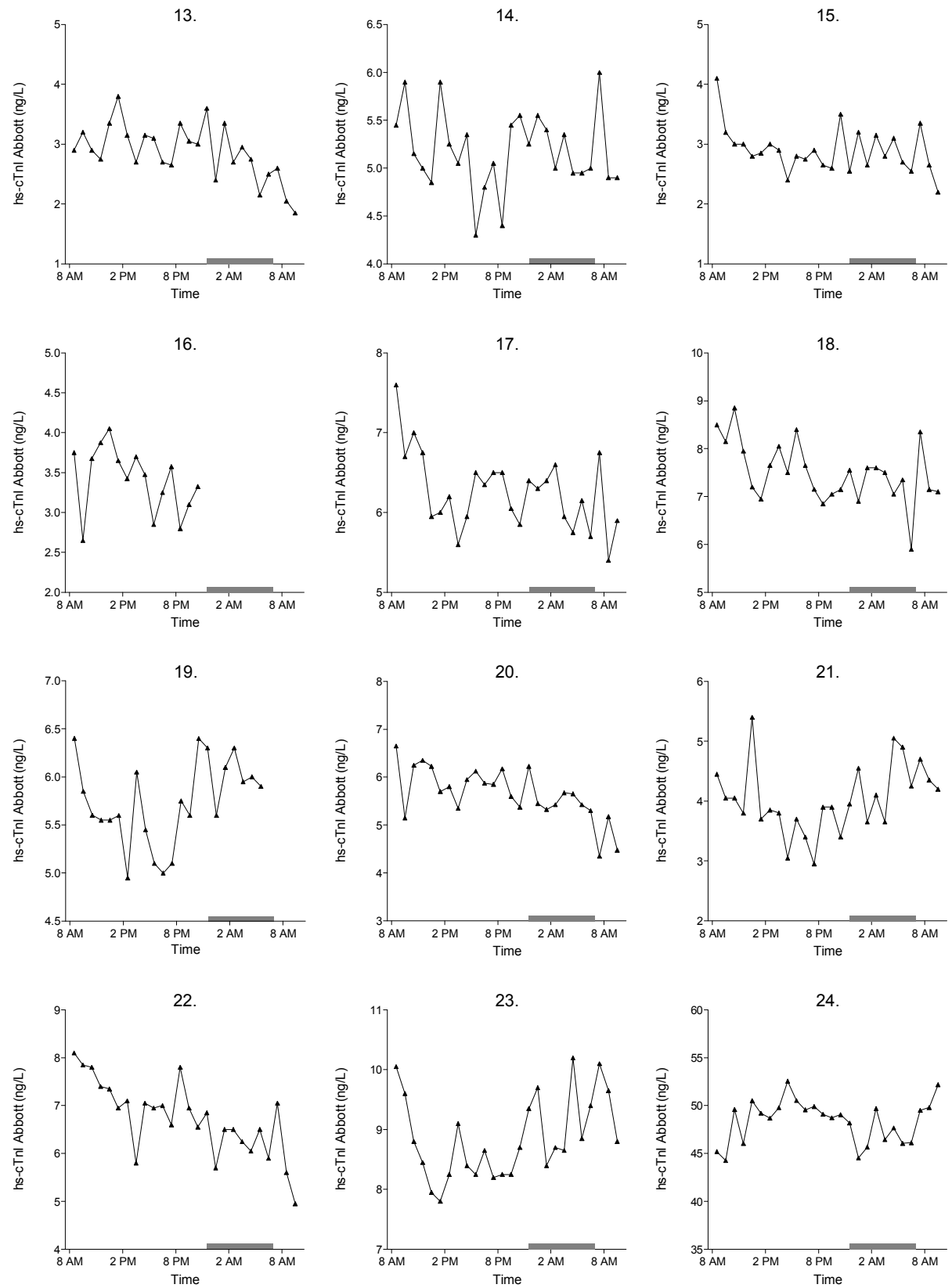

Supplemental figure 5. Diurnal hs-cTnl profiles (continued from previous page).

Depicted are the mean of the duplicate measurements. The numbering is identical to table 1 and supplemental figure 1, 2 and 6. Subjects slept in a supine position between 11:30 PM and 7:00 AM (gray area). Sampling in subjects 2, 3 and 16 was hindered throughout the night due to technical problems with the intravenous cannula. 

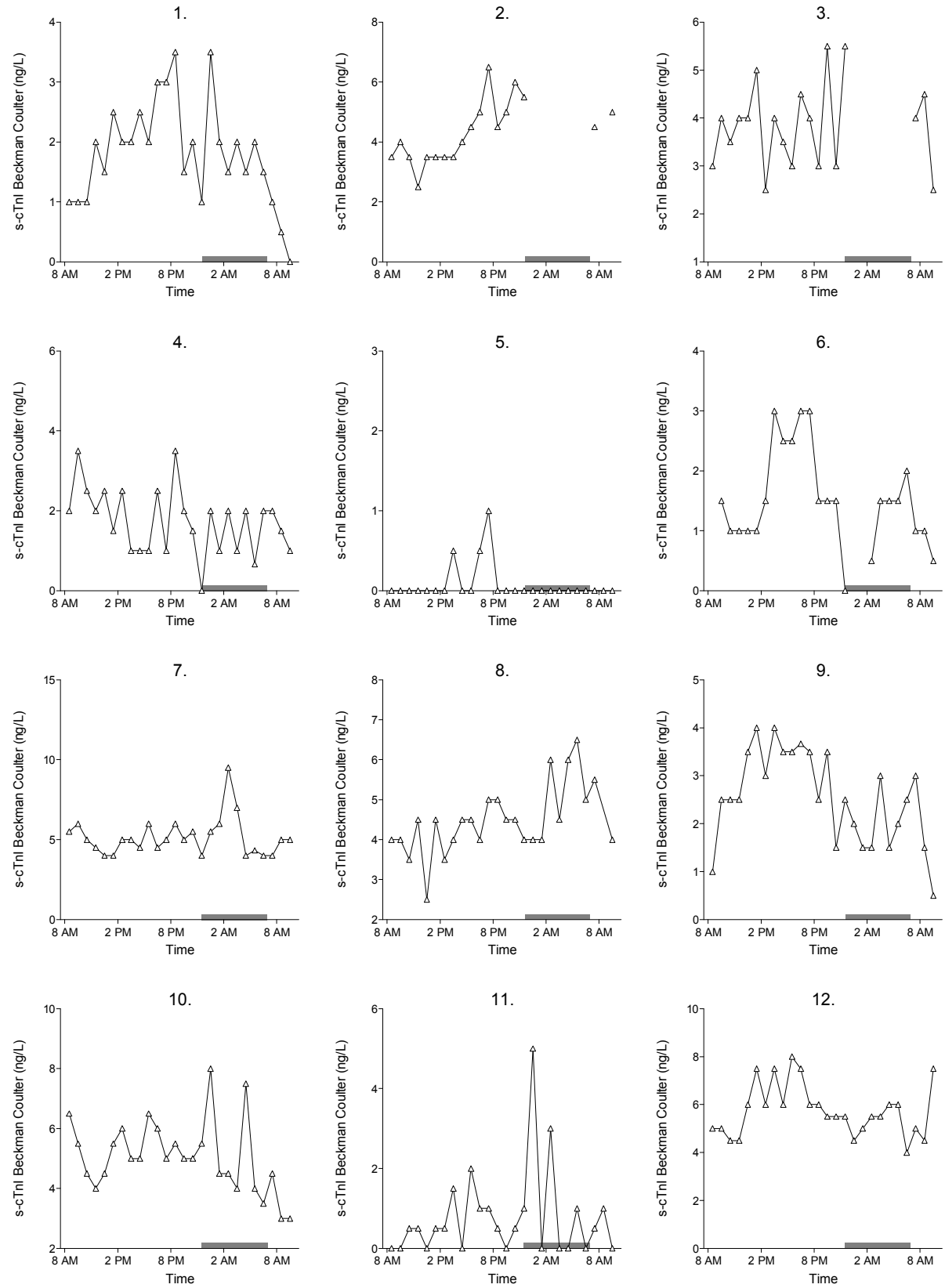

Supplemental figure 6. Diurnal s-cTnl profiles (continued on next page).

Depicted are the mean of the duplicate measurements. The numbering is identical to table 1 and supplemental figure 1, 2 and 5. Subjects slept in a supine position between 11:30 PM and 7:00 AM (gray area). Sampling in subjects 2, 3 and 16 was hindered throughout the night due to technical problems with the intravenous cannula. 

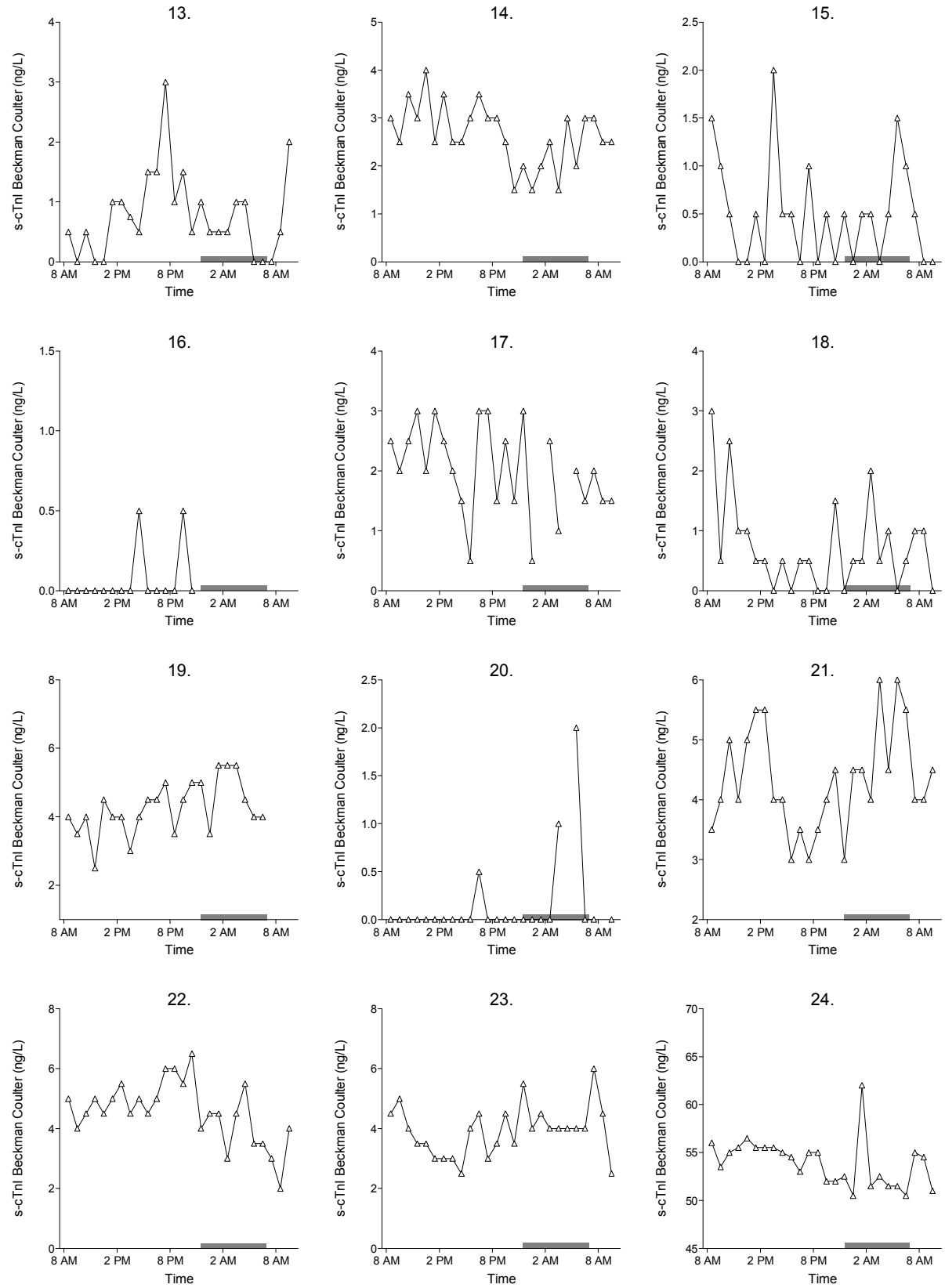

Supplemental figure 6. Diurnal s-cTnl profiles (continued from previous page).

Depicted are the mean of the duplicate measurements. The numbering is identical to table 1 and supplemental figure 1, 2 and 5. Subjects slept in a supine position between 11:30 PM and 7:00 AM (gray area). Sampling in subjects 2, 3 and 16 was hindered throughout the night due to technical problems with the intravenous cannula. 

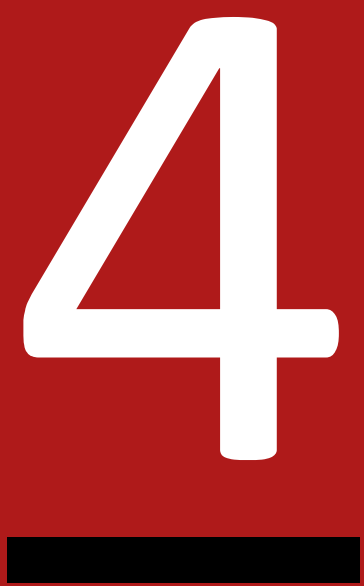


\section{Prognostic value of basal high-sensitive cardiac troponin levels on mortality in the general population: a systematic review and meta-analysis}

Noreen van der Linden, Lieke Klinkenberg, Otto Bekers, Luc van Loon, Marja van Dieijen-Visser, Maurice Zeegers, Steven Meex 


\section{Abstract}

Background The interest in the use of cardiac troponins (cTn) has been expanded from diagnosis of acute myocardial infarction to risk assessment for morbidity and mortality. However, the prognostic value of cTn for all-cause mortality in the general population has not been systematically assessed. In addition, potential differences in the prognostic performance between cardiac troponin T (cTnT) and I (cTnl) are unexplored. The aim of this study was to quantify the relationship between basal levels of high-sensitivity (hs-)cTnT and hs-cTnl and allcause mortality in the general population.

Methods Medline, Embase and the Cochrane Library were searched (from inception through October 2015) for prospective observational cohort studies reporting on the prognostic value of basal hs-cTnT and/or hs-cTnl levels on all-cause mortality in the general population. On a total of 2638 reviewed citations, 39 articles were retrieved, and 9 articles, with data on 43,988 participants, were included in the meta-analysis.

Results Statistical pooling showed a significant association between increased basal cTn levels and an elevated hazard ratio for all-cause mortality during follow-up (HR per 1-SD 1.18 [95\% Cl: 1.09 - 1.27]). Meta-regression analysis showed a trend $(p=0.053)$ towards better prognostic value for $\mathrm{cTnT}$ compared to cTnl (1.31 [95\% Cl: 1.13 - 1.53] and 1.11 [95\% Cl: 1.02 - 1.20], respectively).

Conclusion Elevated, basal CTnT and cTnl levels were significantly associated with increased risk of all-cause mortality during follow-up in the general population. Basal cTnT may better predict all-cause mortality than cTnl. 


\section{Introduction}

Cardiovascular disease kills approximately 17 million people per year, and is the leading cause of death worldwide. ${ }^{1}$ Physicians use risk assessment scores to identify individuals at increased risk for cardiovascular disease and mortality. ${ }^{2}$ Whereas the current algorithms, mainly based on traditional risk factors, do not identify every individual at increased risk, further refinement of these algorithms would advance prognostic medicine. The addition of cardiac troponins (cTn), specific cardiac biomarkers has been proposed. $^{3,4}$

Troponin assays target either cardiac troponin T (cTnT) or I (cTnl), both sarcomere components of the heart. ${ }^{5}$ The release of cTn in the peripheral blood is strongly associated with myocardial injury. ${ }^{6}$ Therefore cTn have become the preferred biomarkers in the diagnostic workup for non-ST elevation acute myocardial infarction (NSTEMI). ${ }^{7,8}$ The recent introduction of high-sensitive (hs) CTn assays has expedited the early diagnosis of NSTEMI, but also resulted in the detection of previously unnoticed levels of cTn in various patient groups without an acute cardiac event such as chronic kidney disease and chronic heart failure. ${ }^{9}$ Subsequently, several studies have shown that these minimal increases of cTnT and cTnl are associated with unfavorable outcomes in these patient groups. ${ }^{10-13}$ Even in apparently healthy subjects cTn can be detected. ${ }^{11}$ This has expanded the use of cTn from acute cardiac care to risk stratification and prognostic medicine. The prognostic value of hs-cTn in apparently healthy subjects from the general population has not been systematically assessed. Also, possible differences in the prognostic performance of hs-cTnT and hs-cTnl are unexplored.

To address these important knowledge gaps, we conducted a systematic review and meta-analysis of the available evidence from published prospective cohort studies. Specifically, we quantified the relationship between basal levels of hs-cTn and all-cause mortality during follow-up in the general population. In addition, the prognostic performance of hs-cTnT and hs-cTnl assays was separately assessed and compared.

\section{Methods}

Data sources and search strategy

Medline, Embase and the Cochrane Library were searched from inception through October 2015. Also the reference lists of all relevant articles and Web of Science (for prospective citations of key publications) were checked for any additional articles. The PubMed search terms were (mortality OR death OR "mortality"[Mesh]) AND (troponin OR "troponin"[Mesh]) AND (predictive OR prediction OR prognostic OR prognosis OR 
"prognosis"[Mesh] risk OR "risk"[Mesh]) AND (follow-up OR prospective OR cohort). We adapted this search strategy for searches of Embase and the Cochrane Library.

\section{Study selection}

Study selection was performed by two independent investigators (N.v.d.L. and L.K.). We included all prospective cohort studies that evaluated the prognostic value of basal hscTnT and hs-cTnl levels in subjects from the general population (without any suspected acute event or surgery at the time of sampling) for all-cause mortality during follow-up.

Studies were excluded from the meta-analysis when A. the duration of follow-up was less than one year, B. hazard ratios (HR) for all-cause mortality were not provided (either as HR per stratum, or as HR per 1-standard deviation (SD) increase in CTn concentrations), or $\mathrm{C}$. when HR were not adjusted for conventional cardiovascular risk factors (at least for age, sex, smoking, hypertension (or systolic blood pressure), diabetes mellitus (or glucose levels) and dyslipidemia (or levels of total and HDL cholesterol). When duplicate publications of data were encountered, only results from the most recent publication were considered. Discrepancies between reviewers were resolved in the presence of a third reviewer (S.M.).

\section{Data extraction and quality assessment}

We extracted data using a customized and validated extraction form. Data on study characteristics (authors, publication year, journal, study design, sample size, country and duration of follow-up), participants' characteristics (age, sex, history of CVD, hypertension, dyslipidemia, diabetes), all-cause mortality rate, cTn assay, HR for all-cause mortality and the factors for which the HR had been adjusted for, were retrieved. When multiple cTn assays of the same kind (cTnl or cTnT) were presented, data from the most commonly used assay in clinical practice was used for our analyses. Two authors (N.v.d.L. and L.K.) performed the data-extraction separately. Discrepancies between reviewers were resolved in presence of a third reviewer (S.M.).

To assess study quality, each study was evaluated for selection bias, study design, confounders, blinding, data collection methods, and withdrawals/dropouts according to the Effective Public Health Practice Project (EPHPP) 'Quality Assessment Tool For Quantitative Studies'. ${ }^{14}$ Two authors (N.v.d.L. and L.K.) performed quality assessment separately, disagreements were resolved by consensus in the presence of a third review author (S.M.)

\section{Data synthesis and analysis}

To quantitatively assess the relationship between basal cTn levels and all-cause mortality during follow-up, we used HR per 1-SD increase in logarithmic transformed cTn levels. When HR were provided for stratified CTn concentrations only, we mathematically 
derived HR per 1-SD increase in logarithmic transformed cTn concentration. This estimation was based on the assumption that logarithmic transformed cTn levels are normally distributed in the population, ${ }^{15-17}$ and consisted of the following three steps: 1 . we extracted the cumulative distribution of the study population and calculated the associated z-scores based on the cumulative standard normal distribution, 2 . we calculated the mean and SD based on the z-scores: $z=(x-\mu) / \sigma$, where $x$ is the log-transformed cTn concentration acting as upper limit of the stratum (continuity correction was performed), $\mu$ is the mean, and $\sigma$ is the SD), and 3. we constructed a biomarker concentration-risk curve ( $x$-axis: log-transformed medians for the strata, $y$-axis: associated HR) for which we calculated the slope (HR/log CTn) and transformed this into HR per 1-SD increase in logarithmic transformed cTn level. This method was validated in two studies that reported HR for both stratified data and log-transformed cTn as a continuous variable. ${ }^{18,19}$ We found a minimal differences between the reported and the calculated HR per 1-SD in these two studies (2 - $8 \%$ difference).

For the meta-analysis, we used full-model adjusted HR (at least adjusted for the conventional cardiovascular risk factors age, sex, smoking, hypertension [or systolic blood pressure], diabetes mellitus [or glucose levels], and dyslipidemia [or levels of total and $\mathrm{HDL}$ cholesterol]). As a result of this approach the number of adjusting factors in the full-model differs between studies. Nevertheless, this method ensures that we correct for all significant confounders in the individual studies. Since cTn are strongly associated with cardiovascular disease, the set of adjusting factors mainly consists of cardiovascular risk factors. The adjusted HRs per 1-SD were pooled using a randomeffects model and summarized using forest plots.

Examination of the impact of potential moderator variables on the study effect size was done by meta-regression analyses. Potential moderator variables included 1 . type of cTn assay, 2. calculated versus reported HRs, 3. study quality, and 4. geographic region. A sensitivity analysis was applied to examine the effect of the inclusion of studies, that did not adjust for the minimal set of six conventional risk factors, on the robustness of the pooled results.

The MOOSE guidelines for meta-analysis of observational studies were followed (supplemental table 1), ${ }^{20}$ and PRISMA criteria were performed for the search methodology (figure 1). Publication bias was evaluated using Egger regression. Heterogeneity between studies was estimated using the $I^{2}$ value. ${ }^{21}$

For all analyses, a 2-tailed p-value less than 0.05 was considered statistically significant. All statistical analyses were performed with the Stata software package (Stata/IC version 13.1). 


\section{Results}

\section{Study characteristics}

The literature search protocol is depicted in figure 1. Our initial search identified 2638 citations. Based on titles and abstracts, 39 articles were considered potentially eligible. After full text evaluation 30 articles were excluded: 11 articles did not report all-cause mortality, 8 articles did not report HR, 4 articles did not report the association between hs-cTn concentrations and all-cause mortality, 4 articles reported data that were also published in more recent articles, 2 studies reported HR that were not adjusted for the minimal list of conventional risk factors, and 1 study was not performed in the general population. Hence, a total of 9 studies with data on 43,988 participants were eventually included in the meta-analysis. ${ }^{16,18,19,22-27}$ Five studies reported HR related to CTnT levels, ${ }^{18,19,22-24}$ another 4 studies reported on cTnl levels. ${ }^{16,25-27}$ Clinical and demographic characteristics are shown in table 1 . The median follow-up duration of all studies was between 3.8 and 14 years. The percentage of individuals with detectable levels of cTn varied from 27 to $96 \%$.

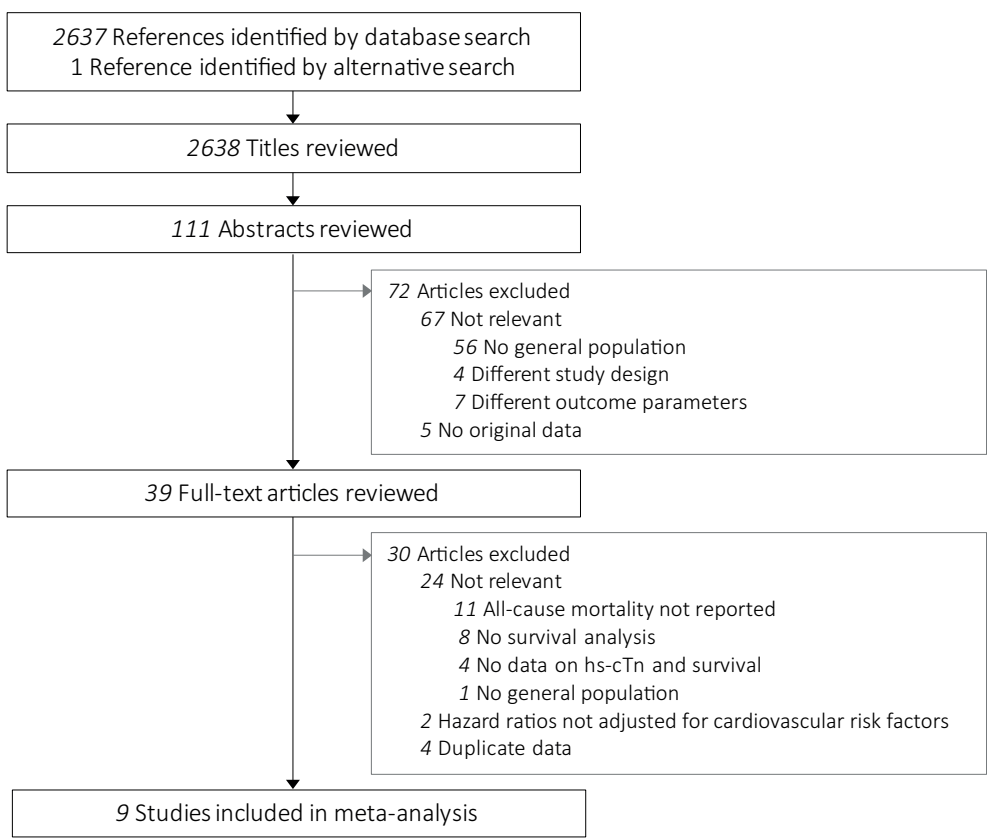

Figure 1. Flow chart of the study selection 


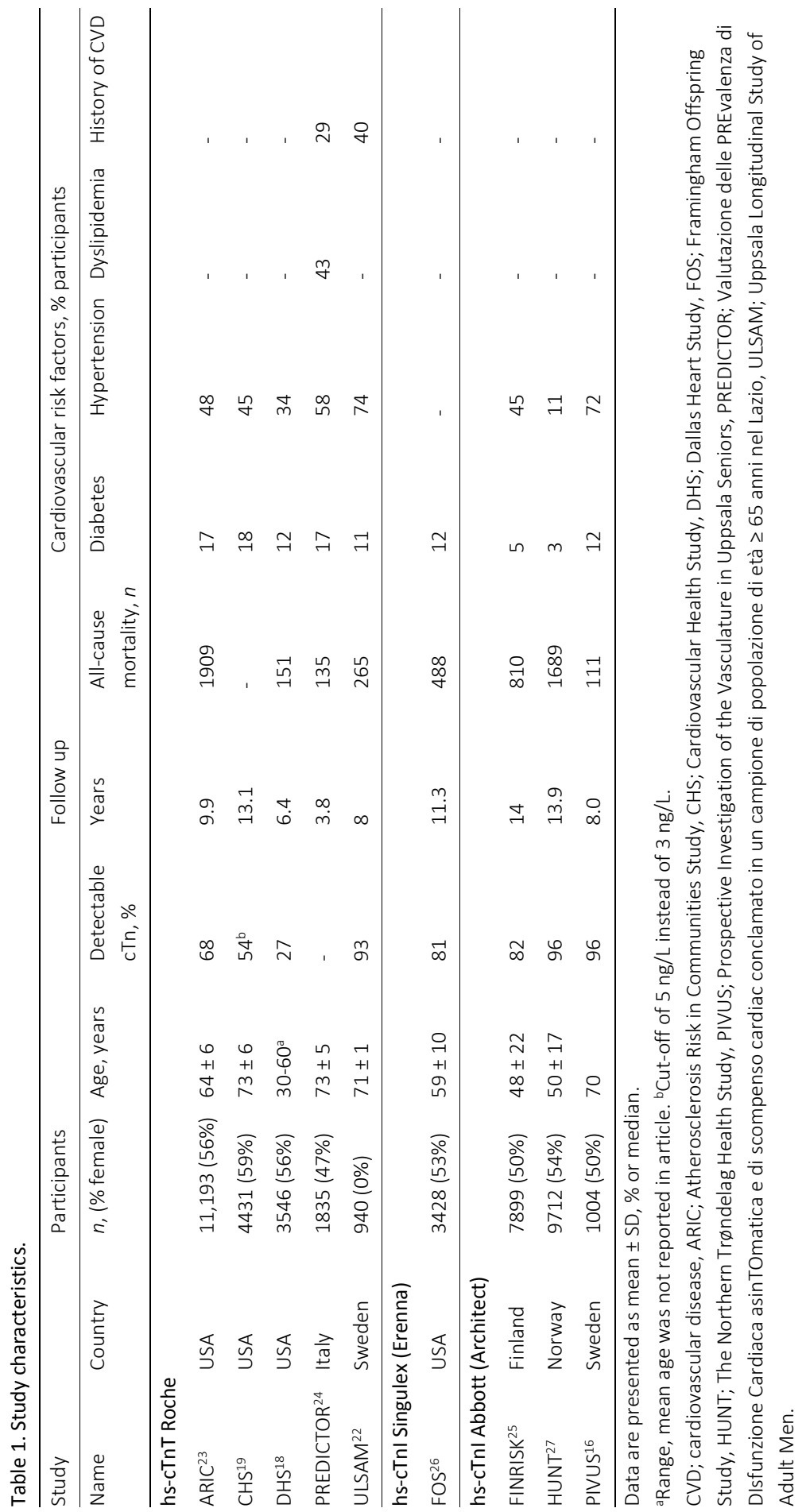


The study quality varied across studies. According to the 'EPHPP quality assessment tool', 14 study was of 'high methodological quality', ${ }^{25} 6$ studies were of 'moderate quality', ${ }^{18,19,22,23,26,27}$ and 2 studies were of 'low methodological quality'. ${ }^{16,24}$ The global rating 'weak' for study quality was due to lack of information about subject inclusion, study withdrawals, and dropouts. To ascertain vital status studies used a death registries $(n=4),{ }^{18,22,24,27}$ medical records $(n=3),{ }^{19,25,26}$ the combination of medical records and a death register $(n=1),{ }^{16}$ and the combination of interviews, medical records and a death register $(n=1) .^{23}$ All included studies provided HR adjusted for at least six conventional cardiovascular risk factors, described in the methods section. Additional adjustments made by the individual studies are presented in supplemental table 2.

\section{Association between hs-cTh and all-cause mortality}

We pooled adjusted HRs per 1-SD for all studies using a random-effects model. Pooling of the results from the nine included studies showed a significant association between increased basal cTn levels and an elevated HR for all-cause mortality (HR per 1-SD 1.18 [95\% Cl: 1.09 - 1.27]). Considering the trend towards different effect sizes (HR per 1-SD) for $c T n T$ and $c T n l$ shown by meta-regression analysis ( $p=0.053)$, we performed stratified analyses for both isoforms of cTn. Basal cTnT levels translated to a higher HR for allcause mortality (HR per 1-SD 1.32 [95\% Cl: 1.13 - 1.53] versus 1.11 [95\% Cl: 1.02 1.20]). The forest plots of the random-effects pooled data are shown in figure 2.

\section{Heterogeneity and publication bias assessment}

Our meta-analysis revealed substantial heterogeneity across studies (figure 2). We used meta-regression analyses to identify the underlying factors. Univariable meta-regression analyses pointed out that estimated versus reported HR, geographic region and study quality (strong/moderate versus weak global rating) were not statistically significant predictors, whereas the assay-type (hs-cTnT or hs-cTnl) approached statistically significance $(p=0.053)$. One SD increase in basal hs-cTnT levels was associated with a higher HR for all-cause mortality during follow-up than a similar increase in hs-cTnl. Stratified analyses for hs-cTnT and hs-cTnl decreased heterogeneity across troponin T studies $\left(\mathrm{I}^{2}=\right.$ $61.7 \%, p=0.034)$, whereas heterogeneity remained high across studies with cardiac troponin I $\left(I^{2}=77.9 \%, p=0.004\right)$.

Egger's regression analysis was not indicative for publication bias $(p=0.340)$. The funnel plot is shown in supplemental figure 1. 
A

Study

DHS

FOS

ARIC

PIVUS

ULSAM

CHS

FINRISK

PREDICTOR

HUNT

Overall

$I^{2}=72.2 \%, p=0.000$
ES $(95 \% \mathrm{Cl})$

$1.43(1.08,1.90)$

$1.06(0.97,1.16)$

$1.39(1.18,1.64)$

$1.21(1.09,1.35)$

$1.12(1.00,1.26)$

$1.16(0.89,1.51)$

$1.01(0.93,1.10)$

$1.77(1.28,2.45)$

$1.17(1.12,1.22)$

$1.18(1.09,1.27)$

$\%$ Weight

5.01
15.12
9.76
13.72
12.95
5.61
15.56
4.09
18.19

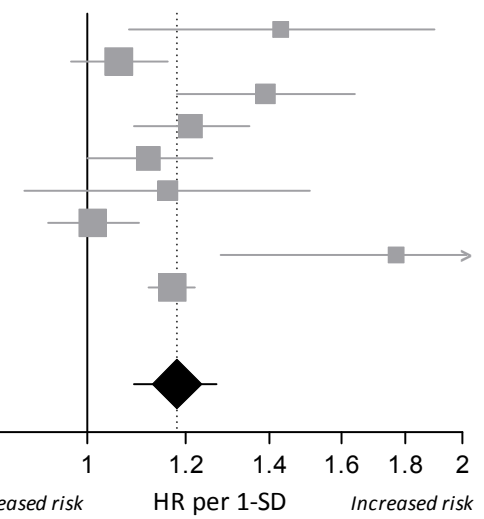

B

Study

ES $(95 \%$ Cl)

DHS

ARIC

ULSAM

CHS

PREDICTOR

hs-cTnT

$r^{2}=61.7 \%, p=0.034$

$1.32(1.13,1.53)$

$1.43(1.08,1.90)$

$1.39(1.18,1.64)$

$1.12(1.00,1.26)$

$1.16(0.89,1.51)$

$1.77(1.28,2.45)$

C

Study
FOS
PIVUS
FINRISK
HUNT

hs-cTnl

$r^{2}=77.9 \%, p=0.004$

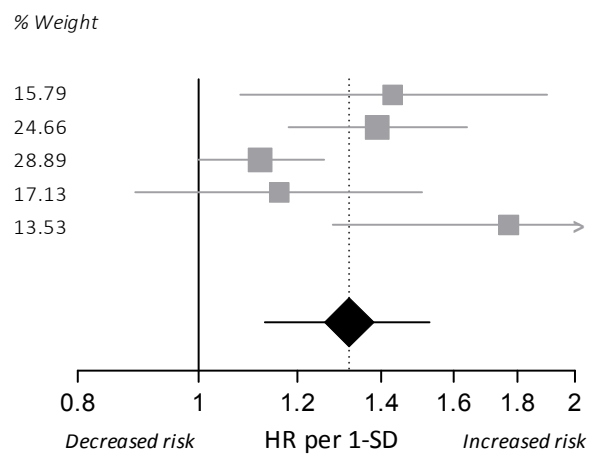

\% Weight

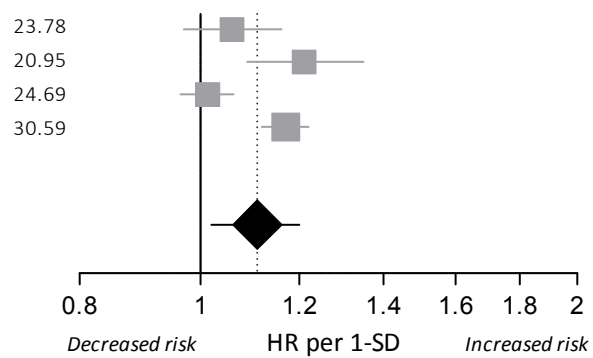

Figure 2. Association between 1-SD increase in basal cTn levels and HR for all-cause mortality in the general population.

The boxes and lines in the forest plots indicate the HR per 1-SD increase in cTn levels for all-cause mortality per individual studies and a pooled estimate (random-effects model) A. for all studies B. studies on hs-cTnT and C. studies on hs-cTnl. 


\section{Sensitivity analysis}

To verify that pooled HR were robust and independent of the exclusion of studies that did not correct for at least the six conventional cardiovascular risk factors, a sensitivity analysis was performed. Random-effects pooling after the addition of two additional studies, ${ }^{28,29}$ revealed similar results and reduced heterogeneity for CTnT (cTnT: HR per 1-SD 1.30 [95\% Cl: $1.16-1.47], \mathrm{I}^{2}=52.9 \%, \mathrm{p}=0.060$, cTnl: HR per 1-SD 1.13 [95\% Cl: $1.11-1.25], I^{2}=65.9 \%, p=0.012$ ) and the combined analysis: HR per 1-SD 1.18 [95\% Cl: $\left.1.11-1.26], I^{2}=64.9 \%, p=0.001\right)$. The meta-regression analysis that was performed after the inclusion of two additional studies, ${ }^{28,29}$ confirmed that assay-type (cTnT vs. cTnl) contributes to the observed heterogeneity $(p=0.022)$.

\section{Translation to clinical practice}

Together, the pooled data show that elevated basal cTn levels in the general population are associated with increased mortality during follow-up, and suggest better prognostic value of cTnT than of cTnl. To relate the pooled summary measures per SD, to a clinically comprehensible risk estimation tool, we transformed HR per 1-SD to absolute CTn concentrations and corresponding HR for all hs-cTn assays that were included in the meta-analysis (figure 3).

A

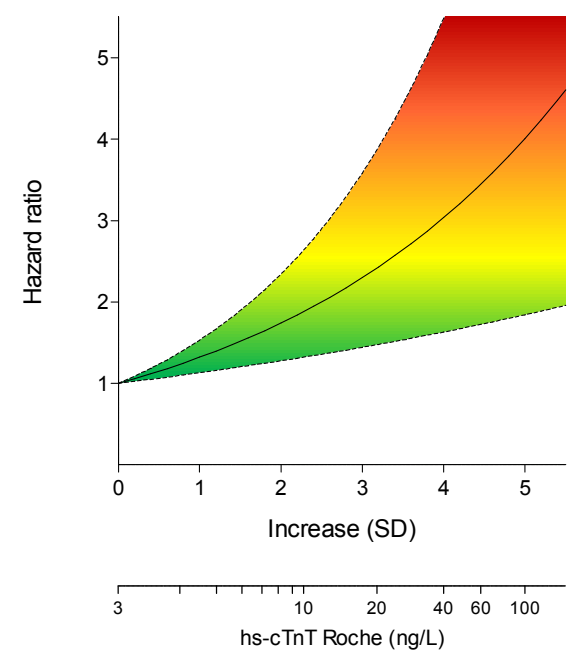

B

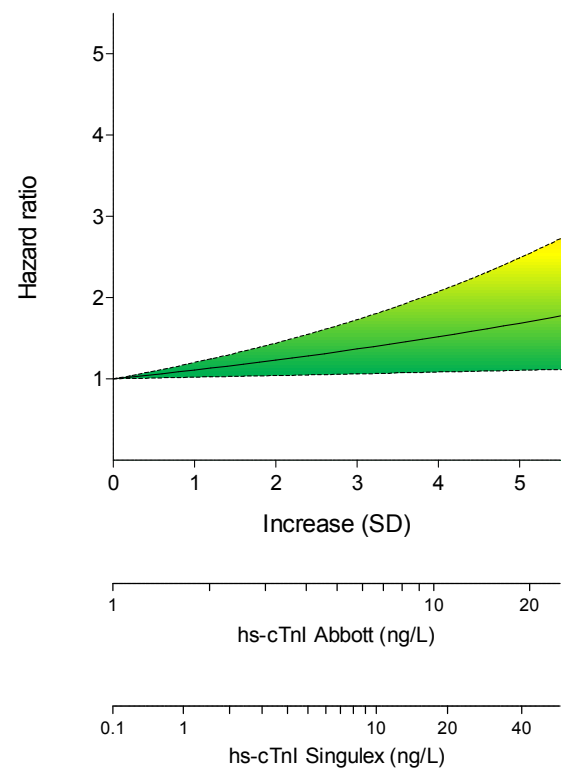

Figure 3. Basal cTnT and cTnl concentrations and corresponding HR.

HR per 1-SD increase was transformed to absolute cTn concentrations and corresponding HR for A. hs-cTnT assays and B. hs-cTnl assays included in the meta-analysis. 


\section{Discussion}

More refined risk stratification models for cardiovascular events and all-cause mortality are of profound importance for primary prevention. This meta-analysis with data on 43,988 participants from 9 prospective cohorts shows that basal elevated cTn concentrations in the general population are associated with an increased risk for all-cause mortality during follow-up. Our pooled data analysis corroborates and extends previous associations in specific patient populations where chronically elevated cTn concentrations are common such as chronic kidney disease. ${ }^{10,12,13}$

This study suggests better prognostic value with less heterogeneity of hs-cTnT than hs-cTnl. Differences in heterogeneity are not surprising: In contrast to cTnl, with dozens of different assays and manufacturers worldwide, the cTnT market has been protected by intellectual property regulations for the last two decades. ${ }^{30}$ The finding that hs-cTnT has better prognostic performance than hs-cTnl is clinically and scholarly interesting, and contrasts with the demonstrated equivalent performance of both assays in the acute setting, i.e. for diagnosing acute myocardial infarction. ${ }^{31}$ We can only speculate about the factors that might underlie these apparent prognostic differences. A first factor concerns the analytical aspects of the distinct cTn assays. It is conceivable that intrinsic assay differences, including the use of different antibodies, ${ }^{32}$ may affect distinctive power of cTn assays with direct consequences for prognostic performance. A second factor involves renal function, which is strongly associated with basal concentrations of $\mathrm{cTn}^{33,34}$ In subjects with an impaired renal clearance, it has been shown that cTnT is more susceptible than cTnl to deteriorations in renal function. ${ }^{35}$ It is not unlikely that the same effect persists in the physiological range of renal function, and confounds the relationship between cTn levels and mortality. A third factor may relate to cTn release itself, a process that is mechanistically unclear. ${ }^{9}$ Possible differences between cTnT and cTnl turnover rate and release mechanisms may affect their respective prognostic performance.

Limitations of our study merit consideration. Despite the consistent adjustment for at least six established cardiovascular risk factors and the use of a standardized outcome measure (HR per 1-SD), heterogeneity was substantial, which persisted in assaystratified analyses, in particular across cTnl studies. Meta-regression analyses did not identify other suggestive factors that contributed to heterogeneity. A likely, remaining source of heterogeneity is the diversity in populations, caused by variable in- and exclusion criteria of the individual studies. Nonetheless, despite the detected heterogeneity, all studies revealed the same direction of effect, with increased mortality upon higher levels of circulating cTn. Another limitation may be the mathematical derivation of HR per 1-SD from stratified data. However, validation of this method in two studies re- 
vealed minimal discrepancies, and this approach to standardize risk-estimates enabled us to pool rather heterogeneously presented data, and to perform a meta-analysis with substantial statistical power and long-term follow-up.

The pooled analysis identifies CTn as an interesting candidate biomarker to further refine the prognostic performance of cardiovascular risk scores. However, before cTnT and/or cTnl can be actually implemented in clinical risk management, knowledge about their performance in metrics of discrimination and reclassification must be expanded. $A$ number of studies showed that addition of cTn levels to risk prediction models based on established risk indicators provided incremental prognostic information regarding allcause mortality and major adverse cardiovascular events. ${ }^{16,22,25-27,29,36,37}$

Future research should focus on further establishment and validation of risk assessment scores by addition of cTn. A second challenge is to confirm and understand the suggestive prognostic differences between cTnT and cTnl.

\section{Acknowledgements}

This study was supported by a research grant from Stichting De Weijerhorst to M.v.D.-V. 


\section{References}

1. Alwan A. Global status report on noncommunicable diseases 2010. Geneva: WHO;2011.

2. De Backer G, Ambrosioni E, Borch-Johnsen K, et al. European guidelines on cardiovascular disease prevention in clinical practice. Third Joint Task Force of European and Other Societies on Cardiovascular Disease Prevention in Clinical Practice. Eur Heart J. 2003;24(17):1601-1610.

3. Wang TJ, Gona P, Larson MG, et al. Multiple biomarkers for the prediction of first major cardiovascular events and death. N Engl J Med. 2006;355(25):2631-2639.

4. Wang TJ. Assessing the role of circulating, genetic, and imaging biomarkers in cardiovascular risk prediction. Circulation. 2011;123(5):551-565.

5. Katrukha IA. Human cardiac troponin complex. Structure and functions. Biochemistry (Mosc). 2013;78(13):1447-1465.

6. de Lemos JA. Increasingly sensitive assays for cardiac troponins: a review. JAMA. 2013;309(21):22622269.

7. Thygesen K, Alpert JS, Jaffe AS, et al. Third universal definition of myocardial infarction. J Am Coll Cardiol. 2012;60(16):1581-1598.

8. Al-Saleh A, Alazzoni A, Al Shalash S, et al. Performance of the high-sensitivity troponin assay in diagnosing acute myocardial infarction: systematic review and meta-analysis. CMAJ Open. 2014;2(3):E199-207.

9. Giannitsis E, Katus HA. Cardiac troponin level elevations not related to acute coronary syndromes. Nat Rev Cardiol. 2013;10(11):623-634.

10. Ahmed AN, Blonde K, Hackam D, lansavichene A, Mrkobrada M. Prognostic significance of elevated troponin in non-cardiac hospitalized patients: a systematic review and meta-analysis. Ann Med. 2014;46(8):653-663.

11. Collinson P. The role of cardiac biomarkers in cardiovascular disease risk assessment. Curr Opin Cardiol. 2014;29(4):366-371.

12. Michos ED, Wilson $\mathrm{LM}$, Yeh $\mathrm{HC}$, et al. Prognostic value of cardiac troponin in patients with chronic kidney disease without suspected acute coronary syndrome: a systematic review and meta-analysis. Ann Intern Med. 2014;161(7):491-501.

13. Khan NA, Hemmelgarn BR, Tonelli M, Thompson CR, Levin A. Prognostic value of troponin T and I among asymptomatic patients with end-stage renal disease: a meta-analysis. Circulation. 2005;112(20):30883096.

14. Thomas BH, Ciliska D, Dobbins M, Micucci S. A process for systematically reviewing the literature: providing the research evidence for public health nursing interventions. Worldviews Evid Based Nurs. 2004;1(3):176-184.

15. Koerbin G, Potter JM, Abhayaratna WP, Telford RD, Hickman PE. The distribution of cardiac troponin I in a population of healthy children: lessons for adults. Clin Chim Acta. 2013;417:54-56.

16. Eggers KM, Venge $P$, Lindahl B, Lind L. Cardiac troponin I levels measured with a high-sensitive assay increase over time and are strong predictors of mortality in an elderly population. J Am Coll Cardiol. 2013;61(18):1906-1913.

17. Venge $\mathrm{P}$, Johnston $\mathrm{N}$, Lindahl B, James S. Normal plasma levels of cardiac troponin I measured by the high-sensitivity cardiac troponin I access prototype assay and the impact on the diagnosis of myocardial ischemia. J Am Coll Cardiol. 2009;54(13):1165-1172.

18. de Lemos JA, Drazner MH, Omland T, et al. Association of troponin T detected with a highly sensitive assay and cardiac structure and mortality risk in the general population. JAMA. 2010;304(22):25032512.

19. Hussein AA, Gottdiener JS, Bartz TM, et al. Cardiomyocyte injury assessed by a highly sensitive troponin assay and sudden cardiac death in the community: the Cardiovascular Health Study. J Am Coll Cardiol. 2013;62(22):2112-2120.

20. Stroup DF, Berlin JA, Morton SC, et al. Meta-analysis of observational studies in epidemiology: a proposal for reporting. Meta-analysis Of Observational Studies in Epidemiology (MOOSE) group. JAMA. 2000;283(15):2008-2012.

21. Higgins JP, Thompson SG. Quantifying heterogeneity in a meta-analysis. Stat Med. 2002;21(11):15391558. 
22. Eggers KM, Al-Shakarchi J, Berglund L, et al. High-sensitive cardiac troponin $\mathrm{T}$ and its relations to cardiovascular risk factors, morbidity, and mortality in elderly men. Am Heart J. 2013;166(3):541-548.

23. Oluleye OW, Folsom AR, Nambi V, Lutsey PL, Ballantyne CM, Investigators AS. Troponin T, B-type natriuretic peptide, C-reactive protein, and cause-specific mortality. Ann Epidemiol. 2013;23(2):66-73.

24. Masson S, Agabiti N, Vago T, et al. The fibroblast growth factor-23 and Vitamin D emerge as nontraditional risk factors and may affect cardiovascular risk. J Intern Med. 2015;277(3):318-330.

25. Neumann JT, Havulinna AS, Zeller T, et al. Comparison of three troponins as predictors of future cardiovascular events - prospective results from the FINRISK and BiomaCaRE studies. PLoS One. 2014;9(3):e90063.

26. Wang TJ, Wollert KC, Larson MG, et al. Prognostic utility of novel biomarkers of cardiovascular stress: the Framingham Heart Study. Circulation. 2012;126(13):1596-1604.

27. Omland $T$, de Lemos JA, Holmen $O L$, et al. Impact of sex on the prognostic value of high-sensitivity cardiac troponin I in the general population: the HUNT study. Clin Chem. 2015;61(4):646-656.

28. Dallmeier D, Denkinger M, Peter R, et al. Sex-Specific Associations of Established and Emerging Cardiac Biomarkers with All-Cause Mortality in Older Adults: The ActiFE Study. Clin Chem. 2015;61(2):389-399.

29. McKie PM, AbouEzzeddine OF, Scott CG, et al. High-sensitivity troponin I and amino-terminal pro-B-type natriuretic peptide predict heart failure and mortality in the general population. Clin Chem. 2014;60(9):1225-1233.

30. Sherwood MW, Kristin Newby L. High-sensitivity troponin assays: evidence, indications, and reasonable use. J Am Heart Assoc. 2014;3(1):e000403.

31. Irfan A, Reichlin T, Twerenbold R, et al. Early diagnosis of myocardial infarction using absolute and relative changes in cardiac troponin concentrations. Am J Med. 2013;126(9):781-788 e782.

32. Apple FS, Collinson PO. Analytical characteristics of high-sensitivity cardiac troponin assays. Clin Chem. 2012;58(1):54-61.

33. Chen S, Huang C, Wu B, Lian X, Mei X, Wan J. Cardiac troponin I in non-acute coronary syndrome patients with chronic kidney disease. PLoS One. 2013;8(12):e82752.

34. Pfortmueller CA, Funk GC, Marti G, et al. Diagnostic performance of high-sensitive troponin T in patients with renal insufficiency. Am J Cardiol. 2013;112(12):1968-1972.

35. Lippi G, Cervellin G. High-sensitivity troponin T is more susceptible than high-sensitivity troponin I to impaired renal function. Am J Cardiol. 2013;112(12):1985.

36. Leistner DM, Klotsche J, Pieper L, et al. Circulating troponin as measured by a sensitive assay for cardiovascular risk assessment in primary prevention. Clin Chem. 2012;58(1):200-208.

37. Zethelius B, Berglund L, Sundstrom J, et al. Use of multiple biomarkers to improve the prediction of death from cardiovascular causes. N Engl J Med. 2008;358(20):2107-2116. 


\title{
Supplemental information
}

\author{
Supplemental tables
}

Supplemental table 1. Moose guidelines checklist

Criteria Brief description of how the criteria were handled in the
meta-analysis

Reporting of the background should include

Problem definition

Hypothesis statement

Description of study outcomes

Type of exposure or intervention used

Type of study designs used

Study population

Reporting of search strategy should include

Qualifications of searchers
We aimed to assess the predictive value of basal highsensitive cardiac troponin levels on all-cause mortality in the general population.

Elevated basal cardiac troponin concentrations are associated with unfavorable outcomes, and thus with an increased hazard ratio for all-cause mortality.

All-cause mortality.

Basal high-sensitive cardiac troponin levels.

Prospective cohort studies.

General population (without any (suspected) acute event or surgery at the time of sampling).

N.v.d.L. (MSc/MD) and L.K. (MSc) were both trained in systematic review and meta-analysis methods as part of their PhD studies. S.M. (PhD) is a clinical chemist, and registered epidemiologist. M.Z. (PhD) is a professor in clinical epidemiology with several years of experience in review methods and statistics, and (co)author of more than thirty systematic reviews and meta-analyses.

Search strategy, including time period included PubMed, Embase and the Cochrane Library were searched in the synthesis and keywords from inception through October 2015. See methodology.

Databases and registries searched PubMed, Embase, the Cochrane Library, and Web of Science.

Search software used, name and version including special features

Use of hand searching

List of citations located and those excluded, including justifications

Method of addressing articles published in languages other than English

We detailed the search in the methods section.

Of all included studies their bibliography and forward citations were examined for further relevant studies.

Details of the literature search are outlined in the flow chart (figure 1).

We were able to obtain all article potentially eligible in English language.

Method of handling abstracts and unpublished In case we would have contacted authors of relevant articles studies for full report of their published studies. We did not attempt to identify unpublished data.

Contact was not made with authors of the studies. 


\section{Criteria}

Brief description of how the criteria were handled in the

meta-analysis

\section{Reporting of methods should include}

Description of relevance or appropriateness of Detailed inclusion and exclusion criteria are described in the studies assembled for assessing the hypothesis methods and in the results section.

to be tested

Rationale for the selection and coding of data Article selection and data extraction were performed by two independent review authors (N.v.d.L. and L.K.), discrepancies in the reviewers' selections were resolved in presence of a third independent reviewer (S.M.). A data extraction form was developed and validated (available on request).

Assessment of confounding We only included studies that reported hazard ratio adjusted for at least six traditional cardiovascular risk factors. We conducted meta-regression analyses for type of cardiac troponin assay, calculated versus retrieved HRs, study quality and geographic region.

Assessment of study quality, including blinding We assessed risk of bias according to the Effective Public of quality assessors; stratification or regression Health Practice Project (EPHPP) 'Quality Assessment Tool For on possible predictors of study results Quantitative Studies'.

Assessment of heterogeneity We used the $\mathrm{I}^{2}$ value to assess heterogeneity.

Description of statistical methods in sufficient detail to be replicated

We reported how hazard ratio's per 1-SD were mathematically derived from stratified data, the type of meta-analyses (random effects model) and the software package used (Stata IC version 13.1).

Provision of appropriate tables and graphics

We included a flow chart (figure 1) to show the process of inclusion, a table showing baseline characteristics of included studies (table 1), and forest plots (figure 2). In addition we added a table with quality assessment scores (supplemental table 2), and a funnel plot graph (supplemental figure 1).

\section{Reporting of results should include}

Graph summarizing individual study estimates Figure 2

and overall estimate

Table giving descriptive information for each

Table 1

study included

Results of sensitivity testing

We conducted a sensitivity analysis, in which we included two studies with hazard ratios that were not adjusted for all six conventional cardiovascular risk factors. Results of sensitivity testing were given in the results section.

Indication of statistical uncertainty of findings

95\% confidence intervals for all calculated outcome measures were provided. 
Criteria

Brief description of how the criteria were handled in the meta-analysis

Reporting of discussion should include

Quantitative assessment of bias

Justification for exclusion

Assessment of quality of included studies

Guidelines for future research

Disclosure of funding source

\section{Reporting of conclusions should include} observations

Generalization of the conclusions
Risk of publication bias was assessed using Egger regression. Results were given in the results section. Funnel plot is depicted in supplemental figure 1.

Reasons for exclusion were reported in the results section and in figure 1.

Studies were graded as weak, moderate or strong according to the Effective Public Health Practice Project (EPHPP) 'Quality Assessment Tool For Quantitative Studies'. Quality assessment was performed by two independent authors (N.v.d.L. and L.K.). Supplemental table 2 shows data on quality assessment.

Consideration of alternative explanations for

In the discussion section we report the heterogeneity in our meta-analyses and the diversity in study populations.

Our conclusions are specific to the use of basal high-sensitive cardiac troponin concentrations in the general population. However, as discussed in the discussion section our findings are consistent with previous observations in various patient populations.

Further research should focus on both the implementation of high-sensitive cardiac troponin in risk assessment scores and on the differences between cardiac troponin $\mathrm{T}$ and $\mathrm{I}$.

Funding statement was provided. 


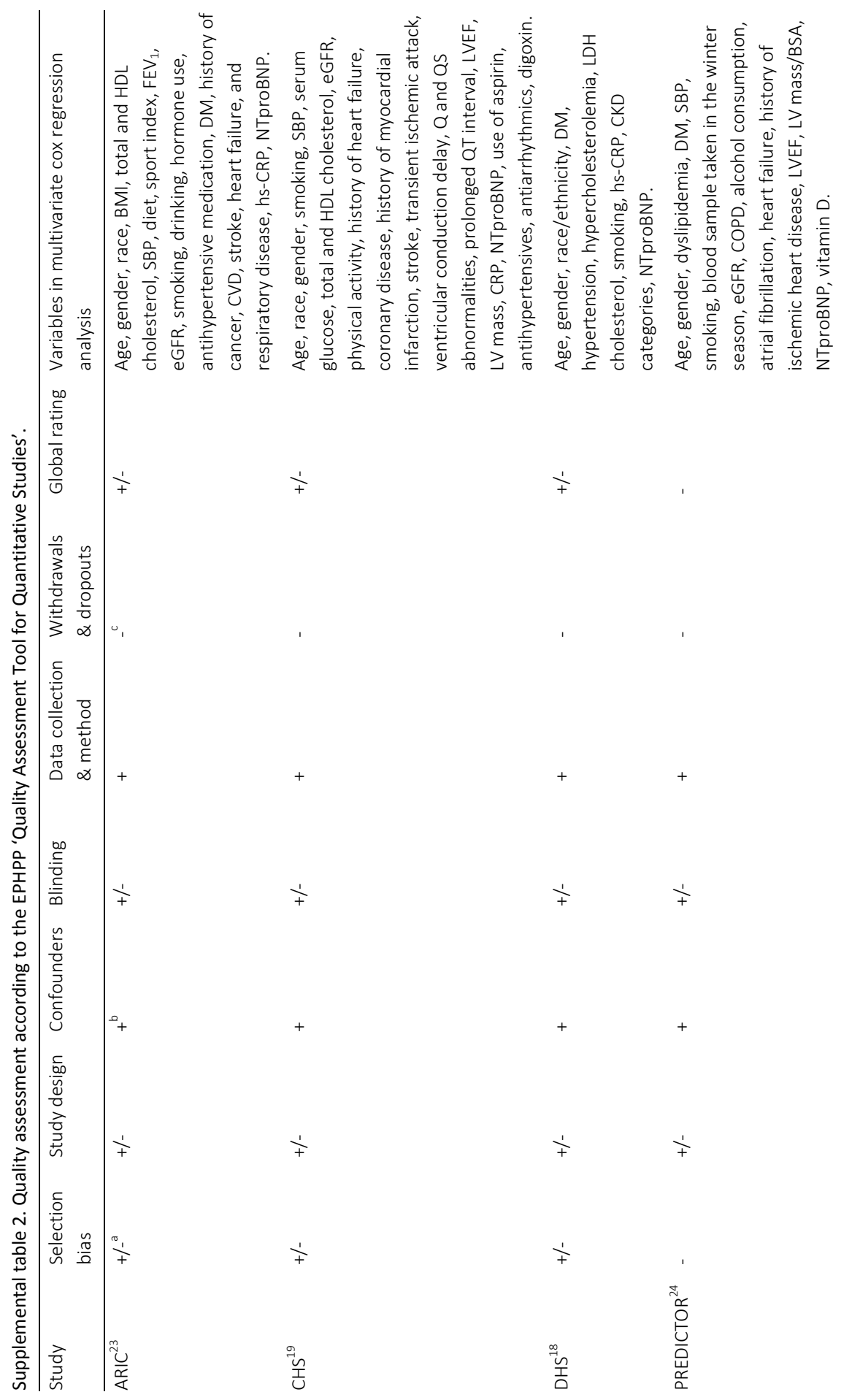



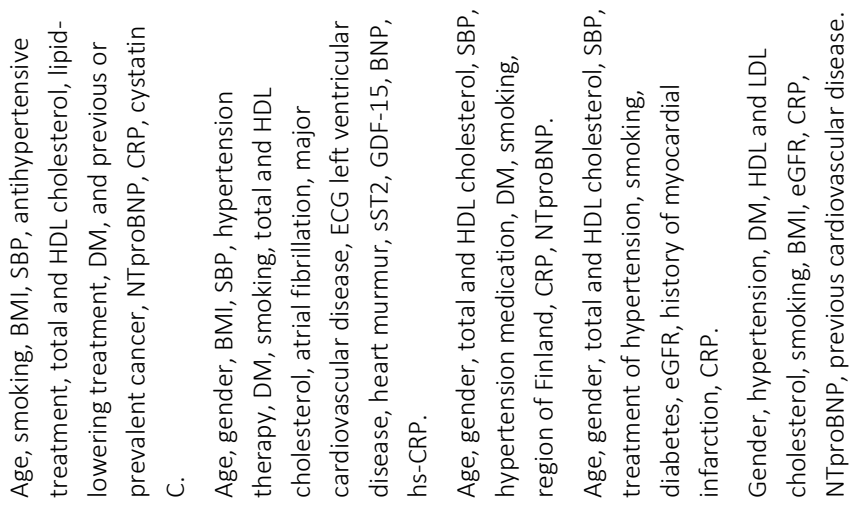

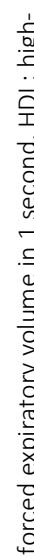
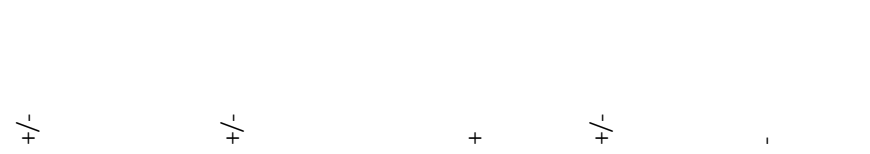

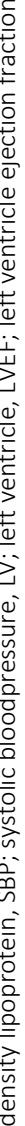


Supplemental figures

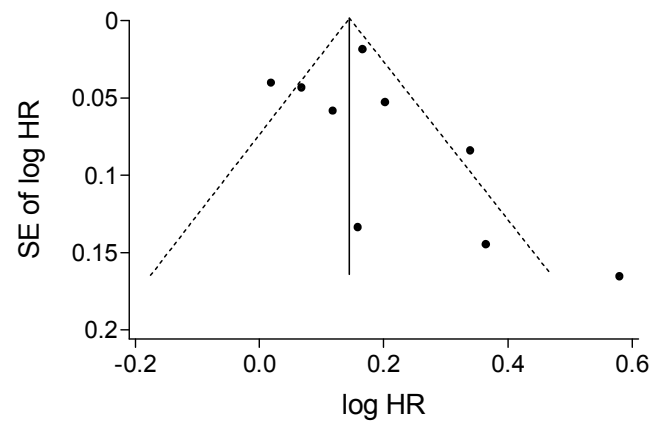

Supplemental figure 1. Funnel plot with pseudo $95 \%$ confidence limits.

The Egger's bias coefficient (coefficient = 1.14, $p=0.34$ ) indicates no publication bias. 



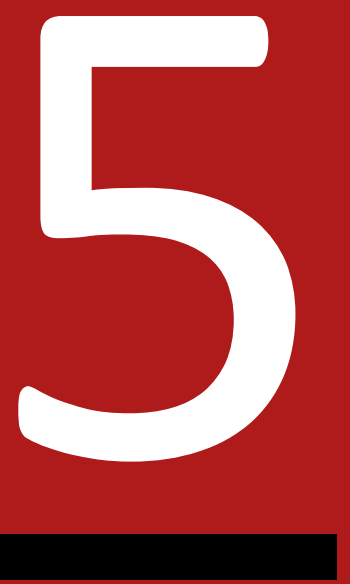




\section{Strong link between basal and exercise- induced cardiac troponin T levels: do both reflect risk?}

Lieke Klinkenberg*, Peter Res*, Luc van Loon, Marja van Dieijen-Visser, Steven Meex International Journal of Cardiology. 2012. 158(1):129-131

* Equal contribution 


\section{Introduction}

Elevated serum high-sensitivity cardiac troponins (hs-cTn) are not restricted to acute coronary syndrome, but are also frequently observed in healthy individuals during and after prolonged endurance-type exercise. ${ }^{1}$ The magnitude of exercise-induced hs-cTn release can vary tremendously among individuals, from virtually no response to more than ten times the diagnostic threshold for acute myocardial infarction (AMI). ${ }^{2}$ Studies to date have examined hs-cTn levels in heterogeneous athlete populations during single or unstandardized ultra-endurance events, complicating the identification of factors involved, or the mechanisms underlying exercise-induced cTn elevations.

\section{Methods}

Using a homogeneous group of endurance-trained athletes, we examined exerciseinduced high-sensitivity cardiac troponin T (hs-cTnT) elevations in a standardized laboratory-based setting to assess the reproducibility, identify predisposing factors and obtain evidence for a physiologic or pathologic nature of this phenomenon. This study was approved by the institutional review board and ethics committee at Maastricht University Medical Center and all participants provided written informed consent. Thirty-one male endurance-trained competitive cyclists (age $25 \pm 5$ years, bodyweight $73 \pm 7 \mathrm{~kg}$, maximal oxygen uptake $\left[\mathrm{VO}_{2} \mathrm{max}\right] 60 \pm 5 \mathrm{~mL} / \mathrm{kg} / \mathrm{min}$, weekly training $11 \pm 4 \mathrm{~h}$ ) completed two identical standardized exercise trials, at a one week interval. The exercise workload was standardized according to the participants' maximal workload capacity (Wmax), as determined in a prior incremental exercise test to exhaustion. The exercise protocol consisted of 60 min steady-state moderate intensity exercise (50\% Wmax) followed by a time-trial, in which athletes performed a set amount of work at maximal performance for about $\approx 60 \mathrm{~min}^{3}$ Blood samples were collected at baseline and immediately after cessation of the time-trial. Serum hs-cTnT was measured using the highsensitivity cTnT assay on the Elecsys 2010 (Roche Diagnostics).

\section{Results}

hs-cTnT concentrations were above the limit of blank ( $3 \mathrm{ng} / \mathrm{L}$ ) in $53 \%$ and $61 \%$ of cyclists before trial 1 and 2, respectively, and in 77\% of cyclists post-exercise in both trials. Immediately after cessation of the time-trial ( $\approx 120 \mathrm{~min}$ ), median hs-cTnT concentrations were significantly increased from baseline in both trials $(p<0.001)$. One $(3 \%)$ and three cyclists (10\%) presented hs-cTnT values above the clinical cut-off for AMI (14 
$\mathrm{ng} / \mathrm{L}$ ) immediately after trial 1 and 2 , respectively. Post-exercise hs-cTnT concentrations were strongly correlated $\left(r^{2}=0.53, p<0.001\right.$, figure 1$)$.

Figure 1. Correlation between post-exercise levels of hs-cTnT assessed in two identical cycling trials. hs-cTnT was measured immediately after trial 1 and 2. The depicted $r^{2}$ does not include two outliers (white circle).

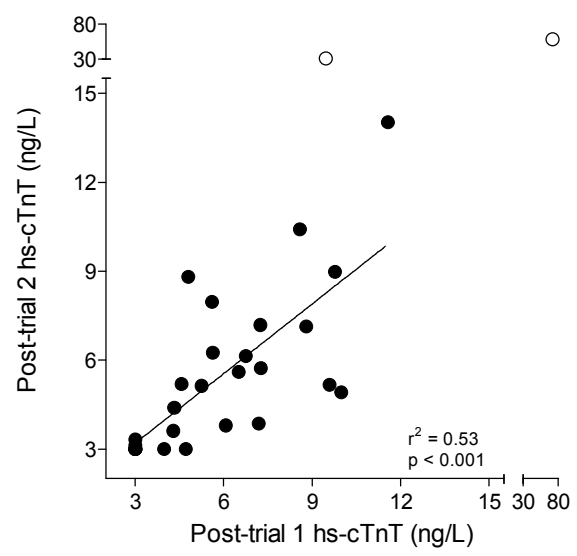

To further assess the reproducibility of hs-cTn release, we categorized individual hs-cTnT changes during both trials as 'elevated' or 'not elevated', using the reference change value (RCV) as an unbiased statistical threshold. A relative increase in serum hsCTnT concentration during exercise of more than $48 \%$ (calculated $\mathrm{RCV}=\mathrm{Z} \cdot \mathrm{V} 2 \cdot \mathrm{CV}_{\text {tot }}$, where $Z=1.65$ and $C V_{\text {tot }}=20 \%$, representing the median coefficient of total variation at baseline), was considered 'elevated'. Twenty-two out of thirty participants presented with concordant hs-cTnT response at both trials, with seven cyclists having an exerciseinduced hs-cTnT elevation above the RCV at both challenges. These numbers translate in a Cohen's Kappa of $k=0.45(p<0.01)$, which, from an epidemiological perspective, represents a good reproducibility.

The very strong correlation and good concordance rate of exercise hs-cTn levels during two identical challenges support the idea that exercise-induced hs-cTnT elevations are not sporadic, but that their occurrence and magnitude are subject to predisposing factors. We performed univariate linear regression analysis and identified two significant predictors of post-exercise hs-cTnT concentration: 1 . baseline hs-cTnT levels $\left(r^{2}=0.80, p<0.001\right.$, figure 2$)$, explaining $80 \%$ of the total variation and 2 . the average power output per kg bodyweight $\left(r^{2}=0.25, p<0.01\right)$. In a multivariate regression analysis, addition of average power output per kg modestly improved the predictive value of the model with baseline hs-cTnT values, explaining $83 \%$ of the total variation $(p<0.001)$. There was no significant association between post-exercise hs-cTnT concentration and age, weekly training, maximal heart rate, $\mathrm{VO}_{2}$ max and Wmax. Other well-established predictors for post-exercise hs-cTnT concentrations such as sex, exercise intensity, 
exercise duration, and training level were not significant predictors in our study since these parameters were either standardized or controlled for in our protocol. ${ }^{4}$

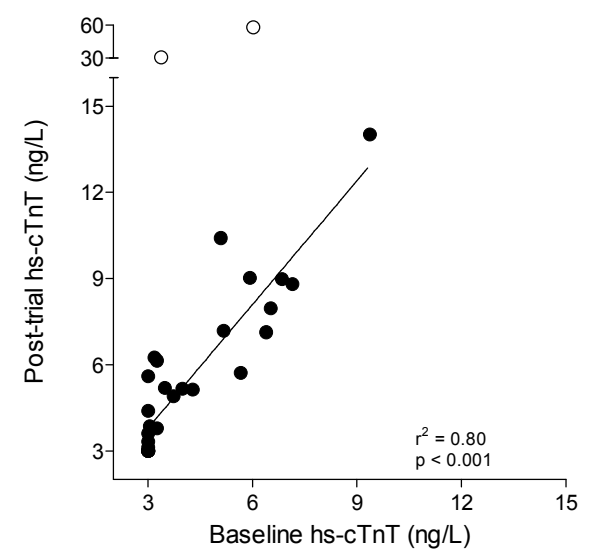

Figure 2. Correlation between baseline and posttrial levels of hs-cTnT.

hs-cTnT was measured at baseline and immediately after the second cycling trial. The depicted $r^{2}$ does not include two outliers (white circle).

\section{Discussion}

The key finding of this study is the strong predictive value of baseline hs-cTnT levels for exercise-induced hs-cTnT concentrations: As much as $80 \%$ of the variation in exerciseinduced hs-cTn levels can be explained by the baseline hs-cTn level. In a broad range of pathologies and patient groups baseline hs-cTn values are repeatedly and robustly associated with adverse cardiovascular prognosis and mortality. ${ }^{5}$ It seems counterintuitive to ascribe exercise-induced hs-cTn elevations to the same mechanism of cardiac necrosis that characterizes AMI, but the observation that variation in exercise-induced hs-cTnT levels almost mirrors the variation in baseline hs-cTn levels, warrants prudence on how high hs-cTn levels after exercise should be regarded. Perhaps, similar to baseline hs-cTn levels, less is better for exercise-induced hs-cTn release.

\section{Acknowledgements}

This study was supported by a research grant from Stichting De Weijerhorst to M.v.D.-V. and a stipendium from the Noyons Stichting and a research grant from Stichting Sint Annadal to S.M. 


\section{References}

1. Middleton N, George K, Whyte G, Gaze D, Collinson P, Shave R. Cardiac troponin T release is stimulated by endurance exercise in healthy humans. J Am Coll Cardiol. 2008;52(22):1813-1814.

2. Mingels A, Jacobs L, Michielsen E, Swaanenburg J, Wodzig W, van Dieijen-Visser M. Reference population and marathon runner sera assessed by highly sensitive cardiac troponin $T$ and commercial cardiac troponin T and I assays. Clin Chem. 2009;55(1):101-108.

3. Jeukendrup A, Saris WH, Brouns F, Kester AD. A new validated endurance performance test. Med Sci Sports Exerc. 1996;28(2):266-270.

4. Shave R, George KP, Atkinson G, et al. Exercise-induced cardiac troponin T release: a meta-analysis. Med Sci Sports Exerc. 2007;39(12):2099-2106.

5. deFilippi $\mathrm{CR}$, de Lemos JA, Christenson RH, et al. Association of serial measures of cardiac troponin $\mathrm{T}$ using a sensitive assay with incident heart failure and cardiovascular mortality in older adults. JAMA. 2010;304(22):2494-2502. 


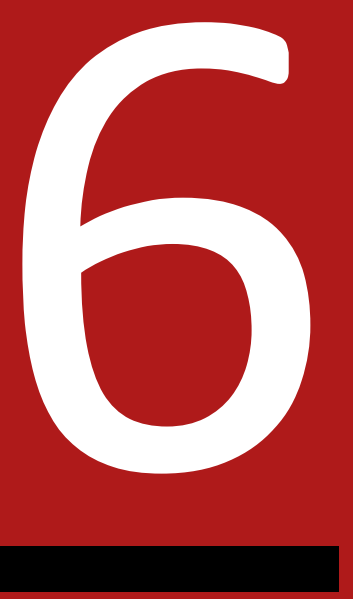




\section{Effect of antioxidant supplementation on exercise-induced cardiac troponin release in cyclists: a randomized trial}

Lieke Klinkenberg*, Peter Res*, Guido Haenen, Aalt Bast, Luc van Loon, Marja van Dieijen-Visser, Steven Meex

PLoS One. 2013. 8(11):e79280

* Equal contribution 


\section{Abstract}

Background Cardiac troponins (cTn) are the biochemical gold standard to diagnose acute myocardial infarction. Interestingly however, elevations of high-sensitivity cardiac troponins (hs-cTn) are also frequently observed during and after endurance-type exercise. Oxidative stress associated with prolonged exercise has been proposed to contribute to cTn release. Therefore, the aim of this study was to assess the effect of 4 week astaxanthin supplementation (a potent carotenoid antioxidant) on antioxidant capacity and exercise-induced cTnT release in cyclists.

Methods Thirty-two well-trained male cyclists (age $25 \pm 5$ years, weight $73 \pm 7 \mathrm{~kg}$, maximum $\mathrm{O}_{2}$ uptake $60 \pm 5 \mathrm{~mL} / \mathrm{kg} / \mathrm{min}$, Wmax $5.4 \pm 0.5 \mathrm{~W} / \mathrm{kg}$; mean $\pm \mathrm{SD}$ ) were repeatedly subjected to a laboratory based standardized exercise protocol before and after 4 weeks of astaxanthin ( $20 \mathrm{mg} /$ day), or placebo supplementation in a double-blind randomized manner. Blood samples were obtained at baseline, at $60 \mathrm{~min}$ of cycling and immediately post-exercise ( $\approx 120 \mathrm{~min}$ ).

Results The pre-supplementation cycling trial induced a significant rise of median hscTnT concentrations from 3.2 (IQR: 3.0 - 4.2) to $4.7 \mathrm{ng} / \mathrm{L}$ (IQR: 3.7 - 6.7), immediately post-exercise $(p<0.001)$. Four weeks of astaxanthin supplementation significantly increased mean basal plasma astaxanthin concentrations from non-detectable values to $175 \pm 86 \mu \mathrm{g} / \mathrm{kg}$. However, daily astaxanthin supplementation had no effect on exercise-induced cTnT release $(p=0.24)$, as measured by the incremental area under the curve. Furthermore, the elevation in basal plasma astaxanthin concentrations was not reflected in changes in antioxidant capacity markers (trolox equivalent antioxidant capacity, uric acid, and malondialdehyde). Markers of inflammation (high-sensitivity Creactive protein) and exercise-induced skeletal muscle damage (creatine kinase) were equally unaffected by astaxanthin supplementation.

Conclusion Despite substantial increases in plasma astaxanthin concentrations, astaxanthin supplementation did not improve antioxidant capacity in well-trained cyclists. Accordingly, exercise-induced hs-cTnT concentrations were not affected by astaxanthin supplementation. 


\section{Introduction}

Myocardial necrosis is accompanied by the release of cardiac troponins (cTn) into the bloodstream, which make cTn the preferred biomarkers for the diagnosis of acute myocardial infarction (AMI). ${ }^{1}$ Interestingly, elevated $\mathrm{cTn}$ are not restricted to AMI, but are also observed in a wide range of cardiac and non-cardiac pathologies, e.g. renal failure, pulmonary embolism, myocarditis and congestive heart failure. ${ }^{2,3}$ In all these pathological settings elevated CTn are consistently associated with poor prognosis. ${ }^{4-7}$ An intriguing example of non-AMI related cTn release in healthy subjects occurs after moderate and highly intensive endurance-type exercise such as marathons, cycling events and long-distance walking. ${ }^{8-12}$ cTn levels in blood rise quickly during exercise, and unlike AMI, they return quickly to normal in approximately $24-48 \mathrm{~h} .{ }^{9,13,14}$

To date, it has not been clarified whether exercise-induced cTn release is a physiologic or pathologic phenomenon. There is no epidemiological evidence thus far that links the magnitude of post-exercise cTn levels to poor prognosis. The faster kinetics of exercise-induced cTn release, and the possible presence of a cytosolic unbound CTn fraction in the cardiomyocyte, ${ }^{15}$ triggered the postulation of a distinct release model for cTn during exercise, unrelated to myocardial cell death. ${ }^{16}$ This hypothetical model states that exercise-induced cTn release could be attributed to increased cardiomyocyte permeability (through mechanical stress or increased production of oxidative radicals) and subsequent early troponin release from a cytosolic cTn pool, possibly through membrane blebbing. ${ }^{16,17}$ This atypical model of benign cTn release was coined the 'reversible injury hypothesis'. It challenges the dogma that increased cTn levels always reflect cell death, and unites two apparently incompatible observations: 1 . the frequent occurrence of exercise-induced cTn release, ${ }^{18}$ and 2 . the limited cardiomyocyte regenerative capacity. ${ }^{19}$ This hypothesis however, has been opposed by a number of observations that warrant prudence before classifying all exercise-induced cTn rises as benign. First, the most pronounced post-exercise cTn concentrations have been observed in the least trained subjects. ${ }^{20}$ Second, baseline cTn levels, which are robust predictors of cardiovascular morbidity and mortality in virtually every patient group investigated, explained up to $80 \%$ of the cTn variation after exercise. ${ }^{21}$ Finally, a strong correlation has been observed between post-exercise cTn and short-term right ventricular functional impairment. ${ }^{22}$ Over time, repetitive strain on the right ventricle may lead to chronic structural changes, as demonstrated by a high prevalence of chronic structural changes in athletes with the highest cumulative exposure to endurance competition. ${ }^{23-25}$

The identification of the biological mechanism that underlies exercise-induced cTn release is an active and relevant topic of discussion. ${ }^{17}$ Oxidative stress associated with prolonged exercise has been proposed to contribute to cTn release. ${ }^{26,27}$ If true, support- 
ing endogenous defense systems with additional doses of antioxidants could therefore be an important strategy to examine the role of oxidative stress as a mechanism for exercise-induced cTn release.

Therefore, we subjected male trained cyclists to a laboratory based standardized exercise protocol before and after 4 weeks of astaxanthin (a potent cartenoid antioxidant) or placebo supplementation. We hypothesized that astaxanthin supplementation attenuates oxidative stress, inflammation and muscle damage following cycling exercise and hence reduces exercise-induced cTn release.

\section{Methods}

This study complies with the Declaration of Helsinki and was approved by the institutional review board and ethics committee at Maastricht University Medical Center. All participants provided written informed consent. This study was registered at clinicaltrials.gov as NCT01241877.

\section{Trial design}

The study was designed as a double-blind, placebo-controlled randomized parallel group trial. Thirty-two healthy male volunteers were recruited between December 2010 and June 2011 via local cycling clubs. We included healthy, young (age between 18 and 30 years), endurance-trained male cyclists, with a training history of more than 3 sessions per week for more than one year. The study was conducted between January 2011 and November 2011 at the Department of Human Movement Sciences at Maastricht University Medical Center. All subjects were competitive cyclists or triathletes who had been involved in regular cycling training (more than 3 sessions per week with $11 \pm 5 \mathrm{~h}$ per week) for at least two years.

\section{Study protocol}

The study was designed to examine the effect of 4 weeks of astaxanthin supplementation on exercise-induced cTn release. This study is part of a greater randomized trial examining the impact of astaxanthin supplementation on muscle metabolism and exercise performance, as primary outcome measures. ${ }^{28}$ Briefly, subjects visited our laboratory on four occasions, two of which were scheduled prior to the start of the main experimental period. The first visit consisted of an incremental cycling exercise test to exhaustion to determine subjects' maximal workload capacity (Wmax) and maximum oxygen uptake $\left(\mathrm{VO}_{2} \mathrm{max}\right)$. The second visit consisted of a familiarization test that was identical in design as the main experimental test days. The actual experimental period (visit 3 and 4) consisted of two identical standardized cycling trials, separated by a 4 
week supplementation period with astaxanthin or placebo. The pre- and postsupplementation trials were performed on the same time and day of the week for each subject. On each test day, subjects reported to the laboratory at 8:00 AM after an overnight fast. A Teflon catheter (Baxter) was inserted into an antecubital vein for blood sampling. The exercise protocol consisted of $60 \mathrm{~min}$ steady-state moderate intensity exercise $\left(50 \%\right.$ Wmax) followed by a validated time-trial, ${ }^{29}$ in which athletes performed a set amount of work at maximal performance for about $\approx 60 \mathrm{~min}$. Blood samples were obtained at baseline, at $60 \mathrm{~min}$ of cycling and immediately post-exercise ( $\approx 120 \mathrm{~min}$ ).

\section{Supplementation protocol}

Participants were randomly assigned in a double-blind fashion to receive either 20 $\mathrm{mg} /$ day of astaxanthin or placebo for 4 weeks. Randomization was performed by a random number generator (www.random.org). Astaxanthin is a xanthophyll carotenoid pigment with potent antioxidative and anti-inflammatory capacities and has previously been shown to attenuate exercise-induced oxidative damage in mouse skeletal and cardiac muscle. ${ }^{30,31}$ The astaxanthin supplementation consisted of a natural astaxanthin extract from the microalga Haematococcus pluvialis ( $80 \mathrm{mg}$ of $5 \%$ astaxanthin) dissolved in sun flower oil in gelatin capsules with added vitamin C (60 mg per capsule) and vitamin E (10 mg per capsule) (BioReal). Participants ingested 2 capsules (4 mg astaxanthin/capsule) with breakfast and 3 capsules with dinner throughout the 4 week intervention period. The placebo supplement was externally identical to the astaxanthin capsule, without the phytonutrient content. The placebo group followed the same supplementation protocol as the astaxanthin group. The supplementation was assigned by an independent investigator, so that both the subjects and investigators were blinded to the treatment. Supplementation compliance was assessed by the number of pills that were left over in the pill bottle after the supplementation period.

\section{Physical activity and dietary standardization}

During the study, subjects were allowed to consume their normal diets but were instructed to refrain from astaxanthin-rich foods such as salmon, lobster, and shrimp. All subjects were also instructed to refrain from physical exercise for two days before each test day. In addition, we asked subjects to record their food intake for two days before the pre-supplementation test day and to replicate their food intake two days before the post-supplementation test day. All subjects received a standardized dinner the evening prior to each experimental test day $(62 \pm 4 \mathrm{~kJ} / \mathrm{kg}$ bodyweight, consisting of 50 energy percent [En\%] carbohydrate, $10 \mathrm{En} \%$ protein, and $40 \mathrm{En} \%$ fat). 


\section{Biochemical analyses}

Plasma and serum samples were stored at $-80^{\circ} \mathrm{C}$ until analysis. Serum cardiac troponin T (cTnT) was measured using the high-sensitivity (hs-) cTnT assay (Roche Diagnostics) on the Elecsys 2010 using lot no. 167650. The measuring range of this assay is $3-10,000$ $\mathrm{ng} / \mathrm{L}$, with a $99^{\text {th }}$ percentile in apparently healthy individuals at $14 \mathrm{ng} / \mathrm{L}$, which is clinically employed to diagnose AMI. ${ }^{32}$ Serum creatine kinase (CK) was measured on Roche Cobas 6000 analyzer. Serum high-sensitivity C-reactive protein (hs-CRP) was measured on the BN Prospec (Siemens). Plasma carotenoid concentrations and antioxidant markers (trolox equivalent antioxidant capacity [TEAC], uric acid, and malondialdehyde [MDA]) have been measured in the setting of a previous study and reported accordingly. ${ }^{28}$

\section{Statistical analysis}

To be able to detect a $10 \%$ reduction in exercise-induced hs-cTnT release (from $7.0 \pm$ 1.4 to $6.3 \pm 1.3 \mathrm{ng} / \mathrm{L}$ ) due to astaxanthin, with a two-sided significance level of $5 \%$ and $80 \%$ power, a total of 32 subjects were recruited. No intervention has been reported that effective reduces exercise-induced cTn release. The effect size of $10 \%$ was therefore derived from the antioxidant-induced reduction of skeletal muscle damage after exercise. $^{33,34}$ In these studies 20 - 30\% reductions of CK levels were obtained through antioxidant supplementation. Assuming a similar mechanism and effect size in cardiac muscle, we felt that a $<10 \%$ reduction would exclude a major role for antioxidants in this setting.

The Kolmogorov-Smirnov test was used to evaluate the normality of the data. Participant characteristics are presented as the mean \pm SD, biochemical parameters are shown as median and interquartile range (IQR). Paired data was tested using the Mann Whitney $U$ test, non-paired data via the Wilcoxon Signed-Rank test. The incremental area under the curve (iAUC) was used as a summary measure for exercise-induced hscTnT release. The effect of antioxidant supplementation was assessed by analysis of variance (ANOVA), using the iAUC at pre-supplementation as covariate. Statistical analysis was performed using SPSS version 18 (IBM SPSS Statistics).

\section{Results}

\section{Participants characteristics}

A detailed flow of the trial is described in figure 1. Thirty-two well-trained cyclists were included in the study. One subject in the placebo group left the study prematurely for medical reasons, unrelated to the study protocol. Subjects' baseline characteristics are shown in table 1 . No differences in age, weight, maximum $\mathrm{O}_{2}$ uptake, maximum work- 
load capacity or the average hours of training per week were observed between the placebo $(n=15)$ and astaxanthin group $(n=16)$.

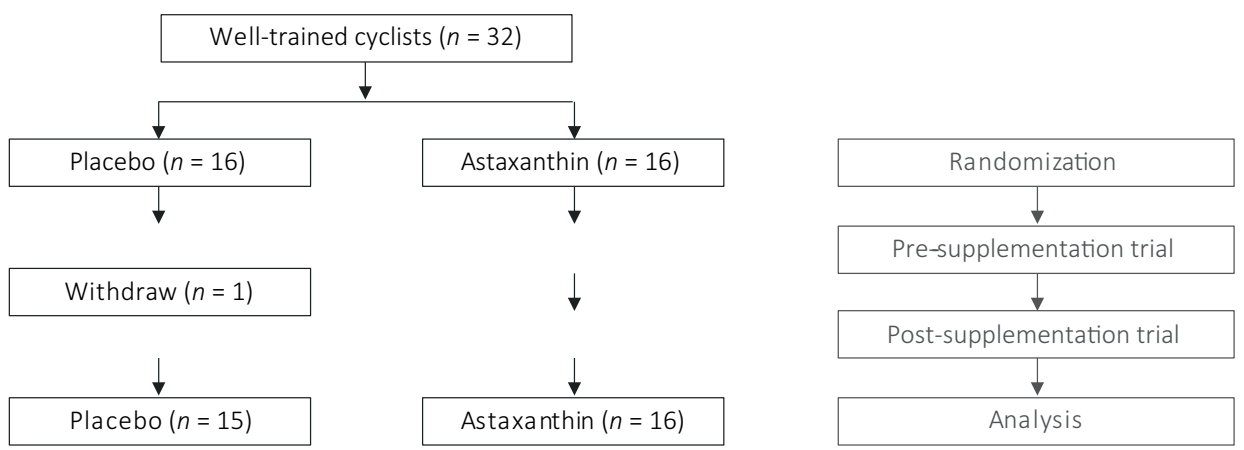

Figure 1. Flow chart of the trial.

\section{Astaxanthin supplementation}

In the placebo group, plasma astaxanthin concentrations were undetectable throughout the study. In the astaxanthin group, plasma astaxanthin concentrations were significantly increased from non-detectable values to $175 \pm 86 \mu \mathrm{g} / \mathrm{kg}$. There were no differences in other carotenoid intake within or between groups during the study.

Table 1. Participants' characteristics.

\begin{tabular}{llll}
\hline & All participants & Placebo & Astaxanthin \\
& $n=31$ & $n=15$ & $n=16$ \\
\hline Age, years & $24.7 \pm 4.7$ & $25.0 \pm 5.5$ & $24.5 \pm 3.9$ \\
Weight, kg & $72.9 \pm 7.2$ & $73.0 \pm 8.5$ & $72.9 \pm 6.1$ \\
Maximum $\mathrm{O}_{2}$ uptake, $\mathrm{mL} / \mathrm{kg} / \mathrm{min}$ & $60.2 \pm 4.8$ & $59.5 \pm 5.4$ & $60.9 \pm 4.3$ \\
Maximum workload, $\mathrm{W} / \mathrm{kg}$ & $5.4 \pm 0.5$ & $5.3 \pm 0.5$ & $5.5 \pm 0.5$ \\
Training, h/week & $10.9 \pm 4.2$ & $10.5 \pm 4.9$ & $11.2 \pm 3.5$ \\
\hline
\end{tabular}

Data are presented as mean \pm SD.

\section{Astaxanthin does not reduce exercise-induced hs-cTnT release}

The pre-supplementation cycling trial induced a significant increase in hs-cTnT, from overall median hs-cTnT 3.2 (IQR: 3.0 - 4.2) to $4.7 \mathrm{ng} / \mathrm{L}$ (IQR: 3.7 - 6.7) immediately postexercise ( $p<0.001$, figure $2 A$ ). Four weeks of astaxanthin supplementation did not affect baseline hs-cTnT concentrations compared to placebo supplementation; median 3.0 (IQR: 3.0 - 4.5) versus $3.7 \mathrm{ng} / \mathrm{L}$ (IQR: 3.0 - 4.6) ( $\mathrm{p}=0.63$ ). The post-supplementation cycling trial resulted in a significant increase from median hs-cTnT 3.0 (IQR: 3.0 - 4.5) to $5.7 \mathrm{ng} / \mathrm{L}$ (IQR: 3.0 - 6.9) in the placebo group (figure 2B), and from median CTnT 3.7 (IQR: 3.0 - 4.7) to $4.8 \mathrm{ng} / \mathrm{L}$ (IQR: 3.6 - 6.9) in the astaxanthin group, immediately post- 
exercise (both $\mathrm{p}<0.01$, figure $2 \mathrm{C}$ ). Despite the increase in basal astaxanthin concentrations, there were no differences between groups in the post-supplementation incremental area under the hs-cTnT curve $(p=0.24)$. Furthermore, there was no significant association between the supplementation-induced astaxanthin concentration and exercise-induced hs-cTnT release (data not shown).

A

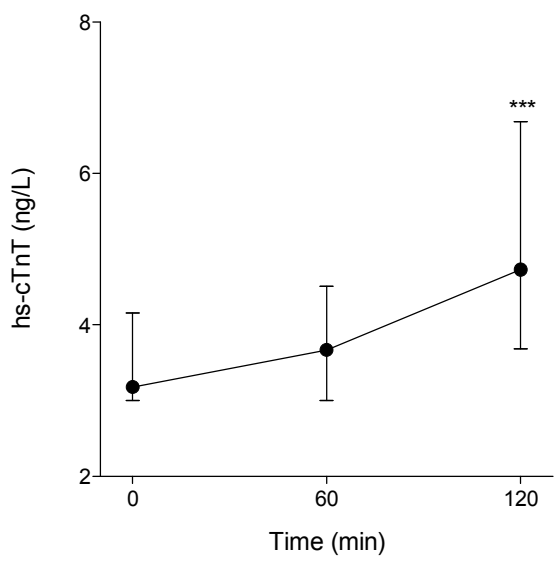

Figure 2. hs-cTnT concentrations during the preand post-supplementation exercise trials.

A. Whole group hs-cTnT concentrations during the pre-supplementation exercise trial. B. hs-cTnT concentrations in the placebo group during both trials. C. hs-cTnT concentrations in the astaxanthin group during both trials. Values are median \pm IQR. ${ }^{* * *}$ denotes a significant increase from baseline $(p<0.001)$.

B

C
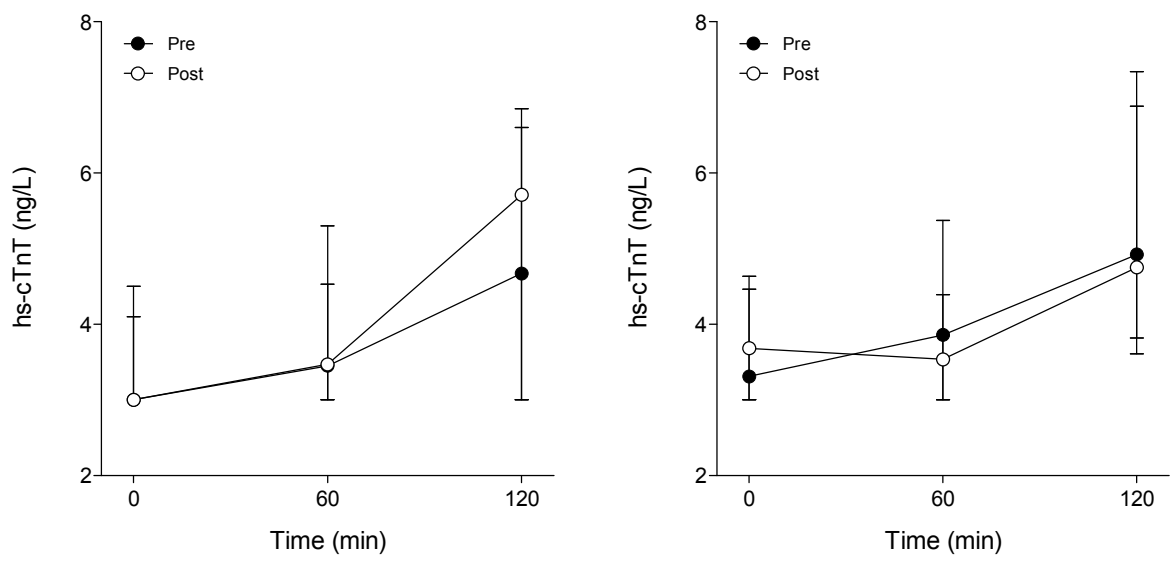


\section{Astaxanthin does not improve antioxidant capacity}

Antioxidant capacity markers including trolox equivalent antioxidant capacity (TEAC), uric acid, and malondialdehyde (MDA) did not differ between the pre- and postsupplementation exercise trials or between groups. Markers of exercise-induced skeletal muscle damage (CK) (figure 3) and inflammation (hs-CRP) (figure 4) were equally unaffected by astaxanthin supplementation. Furthermore, no evident relationship was detected between any of the antioxidant capacity markers and post-exercise hs-cTnT concentrations (supplemental figure 1).

\section{A}

Figure 3. CK concentrations during the pre- and post-supplementation exercise trials.

A. Whole group creatine kinase (CK) concentrations during the pre-supplementation exercise trial. B. CK concentrations in the placebo group during both trials. C. CK concentrations in the astaxanthin group during both trials. Values are median \pm IQR.

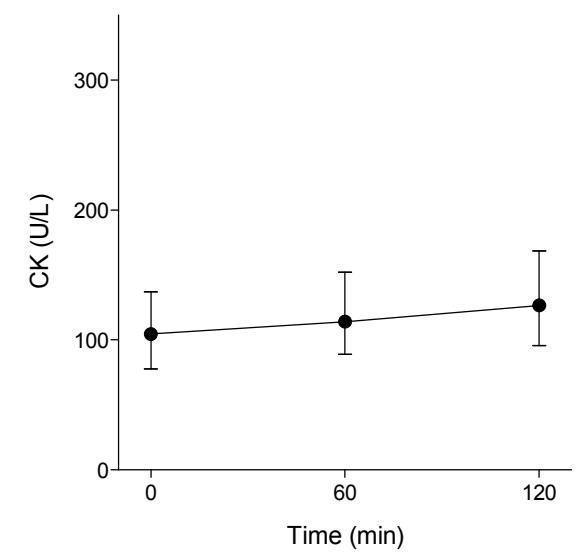

C
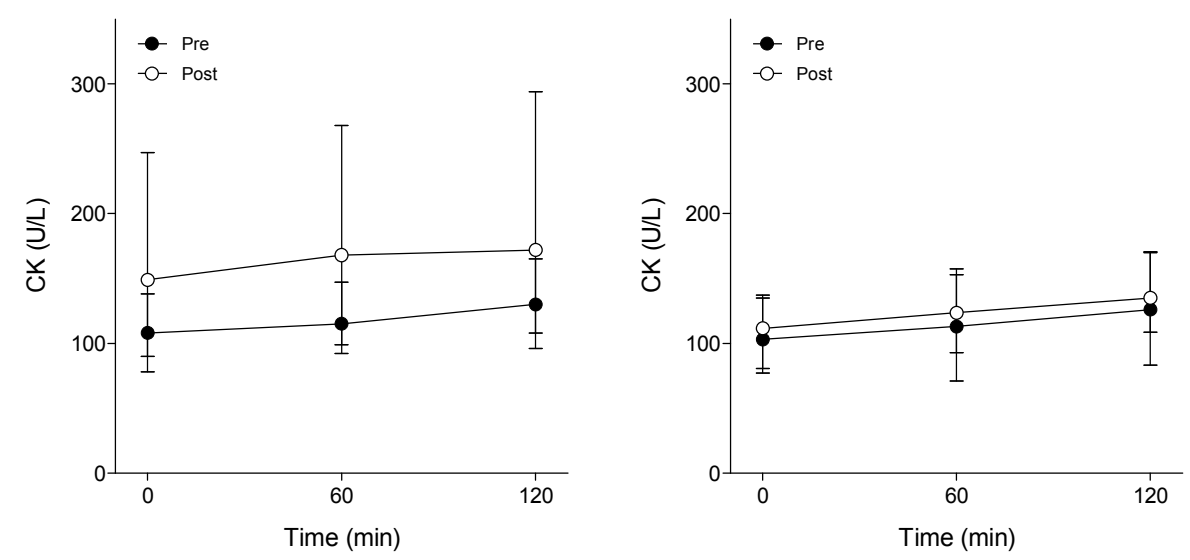
A

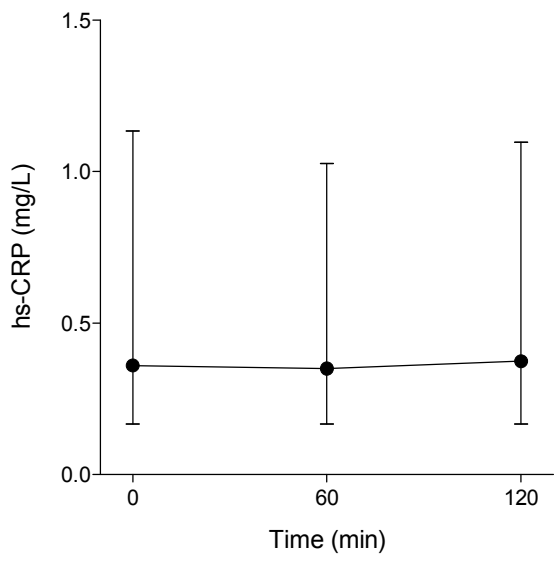

Figure 4. hs-CRP concentrations during the pre- and post-supplementation exercise trials.

A. Whole group high-sensitivity C-reactive protein (hs-CRP) concentrations during the presupplementation exercise trial. B. hs-CRP concentrations in the placebo group during both trials. C. hsCRP concentrations in the astaxanthin group during the pre- and post-supplementation trials. Values are median \pm IQR.

B

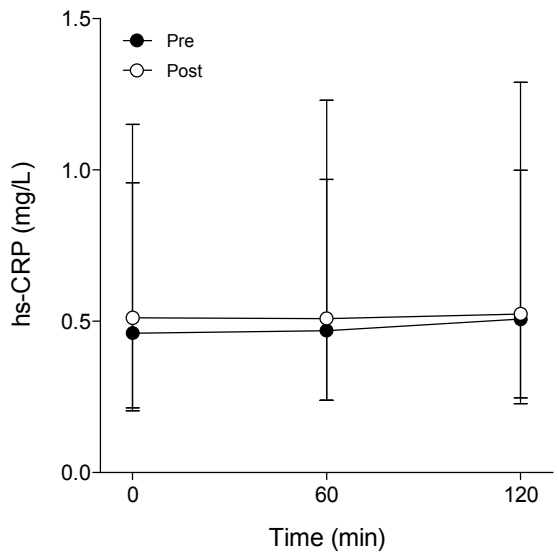

C

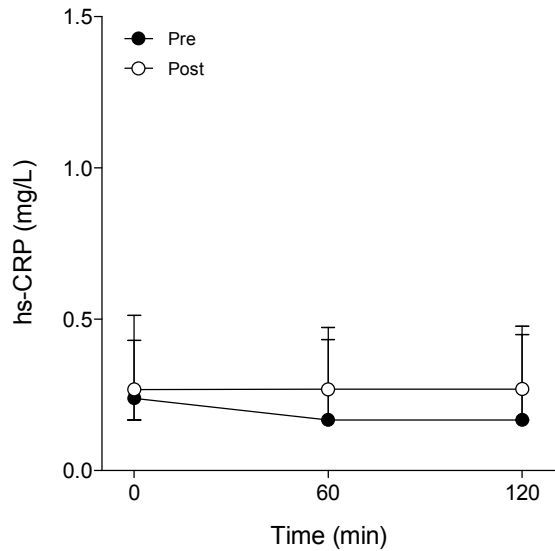

\section{Discussion}

In the present study we demonstrate that 4 weeks antioxidant supplementation did not improve antioxidant capacity, and did not reduce exercise-induced CTnT release in welltrained individuals.

Intensive physical activity is characterized by an increase in oxygen consumption with concomitant production of reactive oxygen species. In the setting of AMI, generation of reactive oxygen species during the reintroduction of blood flow to a previously ischemic area, is a well-established contributor to myocardial reperfusion injury and cTn release. $^{35}$ We hypothesized that a similar mechanism would apply in the setting of 
exercise-induced cTn release, and that oxidative stress associated with endurance exercise causes reversible or irreversible damage to cardiomyocytes with consequent release of cTnT.

The lack of any improvement in antioxidant capacity in the present study was unlikely due to non-compliance of the subjects. Compliance, estimated by pill-counting was $>90 \%$ in 28 out of 31 subjects. Adequate compliance was also evident from strongly increased plasma astaxanthin concentrations. Furthermore, the effect of astaxanthin supplementation was not masked by differences in the total carotenoid intake during the experimental period or between groups. In previous studies, prolonged astaxanthin supplementation (12 weeks, $8 \mathrm{mg} / \mathrm{day}^{36}$ or 12 weeks, $20 \mathrm{mg} / \mathrm{day}^{37}$ ) improved total antioxidant capacity, attenuated MDA and lipid peroxidation. Possibly the highly trained state of our participants, which is associated with already substantially elevated endogenous antioxidant capacity, ${ }^{38,39}$ precluded an additional improvement through antioxidant supplementation.

In line with the absence of any effect of astaxanthin on antioxidant capacity, no significant reduction of exercise-induced CTnT release were observed in subjects following 4 weeks of antioxidant supplementation. To date, one previous study addressed the hypothesis that antioxidant supplementation (tart cherry juice) reduces exerciseinduced cTn release in humans, and concluded that 5 days of antioxidant supplementation did not attenuate circulating cTn following a marathon run. ${ }^{13,40}$ Our study complements this work and deals with some limitations of the original report related to power, duration of antioxidant supplementation, and study design: we extended the period of antioxidant supplementation, increased the number of study subjects and applied a pre-post test design that allows each subject to act as its own control. Our approach circumvents power problems that may arise from the high between-person variation in exercise-induced cTn release, ${ }^{21}$ and exploits the good reproducibility and low withinperson variation of cTn release during two identical exercise bouts. ${ }^{21}$

In conclusion, despite substantial increases in plasma astaxanthin concentrations, astaxanthin supplementation did not improve antioxidant capacity in highly trained cyclists. Accordingly, exercise-induced hs-cTnT concentrations were not affected by astaxanthin supplementation.

\section{Acknowledgements}

The authors gratefully acknowledge the assistance of Vincent Kleijnen. This study was supported by a research grant from Stichting De Weijerhorst to M.v.D.-V. and a research grant from Stichting Sint Annadal to S.M. 


\section{References}

1. Thygesen K, Alpert JS, Jaffe AS, et al. Third universal definition of myocardial infarction. Eur Heart J. 2012;33(20):2551-2567.

2. Agewall S, Giannitsis E, Jernberg T, Katus H. Troponin elevation in coronary vs. non-coronary disease. Eur Heart J. 2011;32(4):404-411.

3. Kelley WE, Januzzi JL, Christenson RH. Increases of cardiac troponin in conditions other than acute coronary syndrome and heart failure. Clin Chem. 2009;55(12):2098-2112.

4. Saunders JT, Nambi V, de Lemos JA, et al. Cardiac troponin T measured by a highly sensitive assay predicts coronary heart disease, heart failure, and mortality in the Atherosclerosis Risk in Communities Study. Circulation. 2011;123(13):1367-1376.

5. van Wijk S, Jacobs L, Eurlings LW, et al. Troponin T measurements by high-sensitivity vs conventional assays for risk stratification in acute dyspnea. Clin Chem. 2012;58(1):284-292.

6. Eggers KM, Venge P, Lindahl B, Lind L. Cardiac troponin I levels measured with a high-sensitive assay increase over time and are strong predictors of mortality in an elderly population. J Am Coll Cardiol. 2013;61(18):1906-1913.

7. Omland T, Pfeffer MA, Solomon SD, et al. Prognostic value of cardiac troponin I measured with a highly sensitive assay in patients with stable coronary artery disease. J Am Coll Cardiol. 2013;61(12):12401249.

8. Neumayr G, Pfister R, Mitterbauer G, et al. Effect of the "Race Across The Alps" in elite cyclists on plasma cardiac troponins I and T. Am J Cardiol. 2002;89(4):484-486.

9. Middleton N, George K, Whyte G, Gaze D, Collinson P, Shave R. Cardiac troponin T release is stimulated by endurance exercise in healthy humans. J Am Coll Cardiol. 2008;52(22):1813-1814.

10. Eijsvogels T, George K, Shave R, et al. Effect of prolonged walking on cardiac troponin levels. Am J Cardiol. 2010;105(2):267-272.

11. Mingels A, Jacobs L, Michielsen E, Swaanenburg J, Wodzig W, van Dieijen-Visser M. Reference population and marathon runner sera assessed by highly sensitive cardiac troponin $T$ and commercial cardiac troponin T and I assays. Clin Chem. 2009;55(1):101-108.

12. Neumayr G, Pfister R, Mitterbauer G, Eibl G, Hoertnagl H. Effect of competitive marathon cycling on plasma $\mathrm{N}$-terminal pro-brain natriuretic peptide and cardiac troponin $\mathrm{T}$ in healthy recreational cyclists. Am J Cardiol. 2005;96(5):732-735.

13. Howatson G, Goodall S, Hill J, et al. Antioxidant supplementation does not attenuate exercise-induced cardiac troponin release. Int J Cardiol. 2011;152(1):101-102.

14. Scherr J, Braun S, Schuster T, et al. 72-h kinetics of high-sensitive troponin T and inflammatory markers after marathon. Med Sci Sports Exerc. 2011;43(10):1819-1827.

15. Katus HA, Remppis A, Scheffold T, Diederich KW, Kuebler W. Intracellular compartmentation of cardiac troponin $T$ and its release kinetics in patients with reperfused and nonreperfused myocardial infarction. Am J Cardiol. 1991;67(16):1360-1367.

16. Hickman PE, Potter JM, Aroney $C$, et al. Cardiac troponin may be released by ischemia alone, without necrosis. Clin Chim Acta. 2010;411(5-6):318-323.

17. Shave R, Baggish A, George K, et al. Exercise-induced cardiac troponin elevation: evidence, mechanisms, and implications. J Am Coll Cardiol. 2010;56(3):169-176.

18. Shave R, George KP, Atkinson G, et al. Exercise-induced cardiac troponin T release: a meta-analysis. Med Sci Sports Exerc. 2007;39(12):2099-2106.

19. Bergmann O, Bhardwaj RD, Bernard S, et al. Evidence for cardiomyocyte renewal in humans. Science. 2009;324(5923):98-102.

20. Mehta R, Gaze D, Mohan S, et al. Post-exercise cardiac troponin release is related to exercise training history. Int J Sports Med. 2012;33(5):333-337.

21. Klinkenberg L, Res PT, van Loon LJ, van Dieijen-Visser MP, Meex SJ. Strong link between basal and exercise-induced cardiac troponin T levels: do both reflect risk? Int J Cardiol. 2012;158(1):129-131.

22. La Gerche A, Burns AT, Mooney DJ, et al. Exercise-induced right ventricular dysfunction and structural remodelling in endurance athletes. Eur Heart J. 2012;33(8):998-1006.

23. Heidbuchel H, Prior DL, La Gerche A. Ventricular arrhythmias associated with long-term endurance sports: what is the evidence? Br J Sports Med. 2012;46 Suppl 1:i44-i50. 
24. Heidbuchel H, Hoogsteen J, Fagard R, et al. High prevalence of right ventricular involvement in endurance athletes with ventricular arrhythmias. Role of an electrophysiologic study in risk stratification. Eur Heart J. 2003;24(16):1473-1480.

25. Wilson M, O'Hanlon R, Prasad S, et al. Diverse patterns of myocardial fibrosis in lifelong, veteran endurance athletes. J Appl Physiol. 2011;110(6):1622-1626.

26. Nie J, Close G, George KP, Tong TK, Shi Q. Temporal association of elevations in serum cardiac troponin T and myocardial oxidative stress after prolonged exercise in rats. Eur J Appl Physiol. 2010;110(6):12991303.

27. Whyte G, George K, Shave R, et al. Impact of marathon running on cardiac structure and function in recreational runners. Clin Sci (Lond). 2005;108(1):73-80.

28. Res PT, Cermak NM, Stinkens R, et al. Astaxanthin supplementation does not augment fat use or improve endurance performance. Med Sci Sports Exerc. 2013;45(6):1158-1165.

29. Jeukendrup A, Saris WH, Brouns F, Kester AD. A new validated endurance performance test. Med Sci Sports Exerc. 1996;28(2):266-270.

30. Aoi W, Naito Y, Sakuma K, et al. Astaxanthin limits exercise-induced skeletal and cardiac muscle damage in mice. Antioxid Redox Signal. 2003;5(1):139-144.

31. Iwamoto T, Hosoda K, Hirano R, et al. Inhibition of low-density lipoprotein oxidation by astaxanthin. J Atheroscler Thromb. 2000;7(4):216-222.

32. Giannitsis E, Kurz K, Hallermayer K, Jarausch J, Jaffe AS, Katus HA. Analytical validation of a highsensitivity cardiac troponin T assay. Clin Chem. 2010;56(2):254-261.

33. Luden ND, Saunders MJ, Todd MK. Postexercise carbohydrate-protein- antioxidant ingestion decreases plasma creatine kinase and muscle soreness. Int J Sport Nutr Exerc Metab. 2007;17(1):109-123.

34. Zoppi CC, Hohl R, Silva FC, et al. Vitamin C and e supplementation effects in professional soccer players under regular training. J Int Soc Sports Nutr. 2006;3:37-44.

35. Park JL, Lucchesi BR. Mechanisms of myocardial reperfusion injury. Ann Thorac Surg. 1999;68(5):19051912.

36. Karppi J, Rissanen TH, Nyyssonen K, et al. Effects of astaxanthin supplementation on lipid peroxidation. Int J Vitam Nutr Res. 2007;77(1):3-11.

37. Choi HD, Youn YK, Shin WG. Positive effects of astaxanthin on lipid profiles and oxidative stress in overweight subjects. Plant Foods Hum Nutr. 2011;66(4):363-369.

38. Gomez-Cabrera MC, Domenech E, Vina J. Moderate exercise is an antioxidant: upregulation of antioxidant genes by training. Free Radic Biol Med. 2008;44(2):126-131.

39. Radak Z, Chung HY, Goto S. Systemic adaptation to oxidative challenge induced by regular exercise. Free Radic Biol Med. 2008;44(2):153-159.

40. Howatson G, McHugh MP, Hill JA, et al. Influence of tart cherry juice on indices of recovery following marathon running. Scand J Med Sci Sports. 2010;20(6):843-852. 


\section{Supplemental information}

\section{Supplemental figures}

A

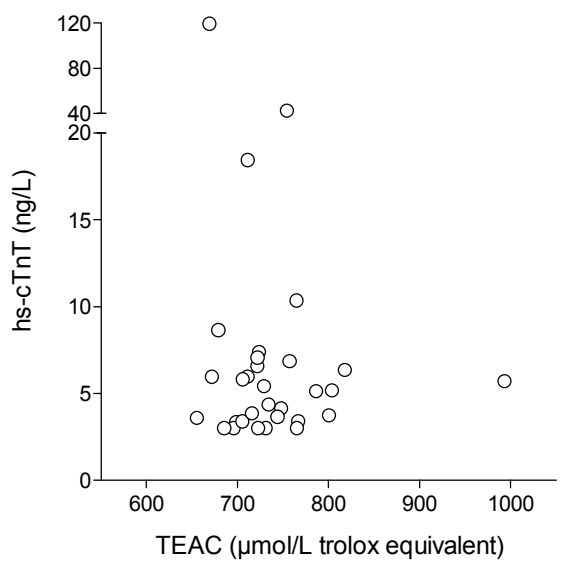

C

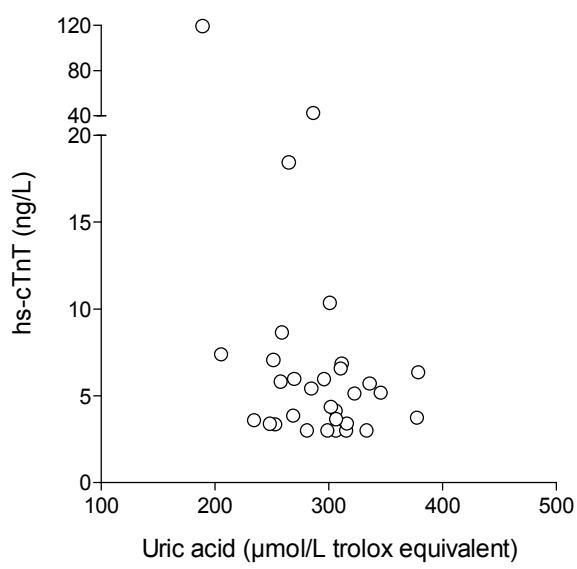

B

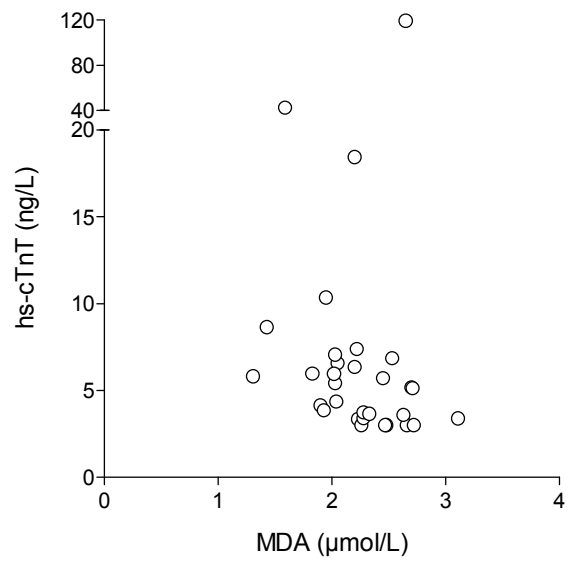

Supplemental figure 1. Relation between hs-cTnT levels and markers of antioxidant capacity.

Post-exercise hs-cTnT concentrations are plotted against post-exercise concentrations of $\mathbf{A}$. trolox equivalent antioxidant capacity (TEAC, B. malondialdehyde (MDA) and $C$. uric acid, all measured during the post-supplementation trial. 



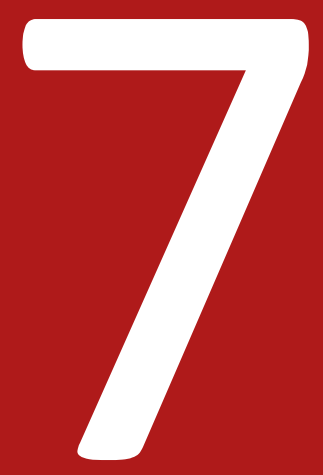




\section{Cardiac troponin $\mathrm{T}$ and I release following a $30 \mathrm{~km}$ run: comparison of troponin kinetics and effect of remote ischemic preconditioning}

Lieke Klinkenberg, Peter Luyten, Noreen van der Linden, Kim Urgel, Daniëlle Snijders, Christian Knackstedt, Robert Dennert, Bastiaan Kietselaer, Alma Mingels, Eline Cardinaels, Frederique Peeters, Jeroen van Suijlen, Joop ten Kate, Elke Marsch, Thomas Theelen, Judith Sluimer, Kristiaan Wouters, Otto Bekers, Sebastiaan Bekkers, Luc van Loon, Marja van Dieijen-Visser, Steven Meex 


\section{Abstract}

Background Prolonged endurance-type exercise is associated with elevated cardiac troponins (cTn) in asymptomatic recreational athletes. It is unclear whether exercise-induced cTn release mirrors a physiological or pathological underlying process. The aim of this study was to provide a direct comparison of the release kinetics of high-sensitivity cardiac troponin I (hs-cTnl) and T (hs-cTnT) following endurance-type exercise. In addition, the effect of remote ischemic preconditioning (RIPC), a cardio-protective strategy that limits ischemiareperfusion injury, was investigated in a randomized controlled crossover manner.

Methods Twenty-five healthy volunteers completed an outdoor $30 \mathrm{~km}$ running trial preceded by RIPC ( $4 \times 5$ min $220 \mathrm{~mm} \mathrm{Hg}$ unilateral occlusion) or control intervention ( $4 \times 5 \mathrm{~min} 20 \mathrm{~mm} \mathrm{Hg}$ unilateral occlusion). hs-cTnT, hs-cTnl and sensitive cTnl (s-cTnl) concentrations were examined before, immediately after, 2 and $5 \mathrm{~h}$ after the trial.

Results The completion of a $30 \mathrm{~km}$ run resulted in a significant increase in circulating cTn (time: all $p<0.001$ ), with maximum hs-cTnT, hs-cTnl and s-cTnl concentrations of $47 \pm 5 \mathrm{ng} / \mathrm{L}, 69 \pm 12 \mathrm{ng} / \mathrm{L}$ and $82 \pm 13 \mathrm{ng} / \mathrm{L}$ (mean \pm SEM), respectively. Maximum hs-cTnT concentrations were measured in $60 \%$ of the participants at $2 \mathrm{~h}$ post-exercise, compared to hs-cTnl and s-cTnl maximum concentrations at $5 \mathrm{~h}$ in $84 \%$ and $80 \%$ of the participants. Application of a RIPC stimulus did not reduce exercise-induced cTn release (time $x$ trial: all $p>0.5$ ).

Conclusion In contrast to acute myocardial infarction, maximum hs-cTnT levels postexercise precede maximum hs-cTnl levels. Distinct release kinetics of hs-cTnT and hs-cTnl, and the absence of an effect of RIPC, favors the concept that exercise-induced cTn release may be unrelated to ischemia-reperfusion injury and mechanistically distinct from cTn release in acute myocardial infarction. 


\section{Introduction}

Cardiac troponins (cTn) are a sensitive markers for the detection of myocardial injury. ${ }^{1}$ Blood levels of cTn, either measured by cardiac troponin I (cTnI) or T (cTnT) assays, are invariably associated with increased rates of cardiovascular events and mortality in a wide range of cardiac and non-cardiac diseases. ${ }^{2}$ The single exception where levels of cTn have not been linked to poor prognosis is the release of cTn during and after endurance-type exercise. ${ }^{3}$ To date, it is unclear whether exercise-induced cTn release mirrors a physiological or pathological underlying process. ${ }^{4,5}$

Exercise-induced cTn release is a common and reproducible phenomenon in endurance-type exercise of various intensities, from long distance walking to competitive running, cycling or triathlon events. ${ }^{5-9}$ Athletes often exhibit post-exercise peak cTn values that exceed the clinical threshold for the diagnosis of acute myocardial infarction (AMI), but without experiencing symptoms of myocardial ischemia. ${ }^{8}$ Normalization of these elevated cTn levels occurs usually within 24 - 48h upon completion of the exercise bout, ${ }^{10,11}$ which is much faster than the normalization after AMI (4 - 14 days). ${ }^{12}$

Insight into the (patho-)physiology of exercise-induced cTn release is an active and relevant topic of discussion. ${ }^{5}$ The recent advent of both a high-sensitivity (hs-) assay for cTnl and cTnT with similar analytical characteristics, enables to directly compare the kinetics of both diagnostic equivalent molecules. For example in AMI, where cTn release results from the breakdown of the contractile apparatus following ischemic myocardial injury, peak levels of hs-cTnl and hs-cTnT are reached at similar levels postadmission. ${ }^{13,14}$ A direct comparison of exercise-induced hs-cTnl and hs-cTnT release could provide more insight into the underlying pathophysiology. In addition, remote ischemic preconditioning (RIPC), a powerful non-invasive cardio-protective strategy, can be applied to study the contribution of an imbalance between oxygen supply and demand in exercise-induced cTn release. RIPC describes the application of brief episodes of non-lethal ischemia and reperfusion to a tissue or an organ, resulting in protection of the same or another visceral organ against an injurious ischemic insult in the future. ${ }^{15,16}$ In line with the cardioprotective effect of RIPC in various settings of tissue ischemia, we hypothesize that if an oxygen demand-supply imbalance contributes to exerciseinduced CTn release, application of a RIPC stimulus would result in reduced postexercise cTn levels.

The aim of this study is a head-to-head comparison of the release kinetics of hscTnT and hs-cTnl following prolonged endurance-type exercise. In addition, we examined the effect of RIPC on exercise-induced cTn release using a randomized controlled single-blind crossover design. 


\section{Methods}

Subjects

Twenty-nine healthy runners (age range: 18 - 65 years) were recruited with posters at local running clubs. Before testing, all participants were informed about the study procedures and methodology, but remained naïve to the study rationale. This study complied with the Declaration of Helsinki and was approved by the institutional review board and ethics committee of Maastricht University Medical Center. All participants provided written informed consent. This study was conducted between March 2013 and June 2013 and was registered at clinicaltrials.gov as NCT01774461.

\section{Experimental design}

In a randomized controlled single-blind crossover design, participants completed two identical outdoor $30 \mathrm{~km}$ running trials, either preceded by a RIPC or control intervention. Both experimental test days started in the morning and were separated by at least two weeks. Before each trial, participants were instructed to refrain from any strenuous physical labor and sports activities for $24 \mathrm{~h}$ and to standardize their breakfast on both days. The trials were organized in a research setting on paved (bicycle) lanes with a minimal number of cross sections with the start and finish $\approx 1 \mathrm{~km}$ from Maastricht University Medical Center. While running, participants were individually followed by a researcher on a bicycle and allowed to drink water and isostar sports drink ad libitum.

\section{Remote ischemic preconditioning}

Temporal unilateral upper arm ischemia was achieved by inflating a blood pressure cuff to $220 \mathrm{~mm} \mathrm{Hg} .{ }^{17}$ Circulatory occlusion lasted $5 \mathrm{~min}$ and was followed by $5 \mathrm{~min}$ of reperfusion by deflation of the cuff. This sequence was repeated for a total of four cycles and resulted in a total procedure time of $40 \mathrm{~min}$ ( $4 \times$ [ $5 \mathrm{~min}$ of circulatory occlusion $+5 \mathrm{~min}$ non-occlusion]). The control intervention followed an identical protocol, except for the blood pressure cuff being inflated to only $20 \mathrm{~mm} \mathrm{Hg}$, allowing uninterrupted perfusion. The order of testing was randomized and counterbalanced between participants.

\section{Biochemical analysis}

Blood samples were collected in serum and EDTA-containing tubes at baseline (preceding the RIPC intervention), immediately post-exercise, 2 and $5 \mathrm{~h}$ post-exercise. Within $1 \mathrm{~h}$ of collection, hematology parameters were analyzed on a Sysmex XE-5000 analyzer. In addition, serum tubes were centrifuged and aliquots were stored at $-80^{\circ} \mathrm{C}$ until analysis. Serum cTnT was measured using the high-sensitivity assay of Roche Diagnostics; the clinical reference limit $\left(99^{\text {th }}\right.$ percentile of a healthy reference population) is $14 \mathrm{ng} / \mathrm{L}$, with a $10 \%$ analytical variation at $13 \mathrm{ng} / \mathrm{L}^{18}$ Serum cTnl was measured with two assays: 
1. the STAT high-sensitivity troponin I assay (Abbott Diagnostics). According to the manufacturer, the $99^{\text {th }}$ percentile limit is $26.2 \mathrm{ng} / \mathrm{L}$ with a corresponding coefficient of variation of $4 \%$. 2. the Access AccuTnl+3 Assay (Beckman Coulter); with a $99^{\text {th }}$ percentile limit of $40 \mathrm{ng} / \mathrm{L}$ and a corresponding 10\% imprecision, as specified by the manufacturer. Complementary cardiovascular or skeletal biomarkers; N-terminal pro-B-type natriuretic peptide (NTproBNP), creatine kinase (CK), creatine kinase muscle and brain fraction (CK$\mathrm{MB}$ ) and lactate dehydrogenase (LD) were measured using assays of Roche Diagnostics. In addition, creatinine (Roche Diagnostics) and cystatin C (Gentian) were measured to calculate the estimated glomerular filtration rate (eGFR) using the CKD-EPI formula. ${ }^{19}$

\section{Statistical analysis}

Sample size estimation was based on hs-cTnT as the primary outcome variable. We aimed to include 29 runners to detect a 30\% reduction in exercise-induced hs-cTnT release due to RIPC (from $40 \pm 32 \mathrm{ng} / \mathrm{L}$ to $28 \pm 32 \mathrm{ng} / \mathrm{L}$, mean $\pm \mathrm{SD}$ ) with a statistical power of 0.8 at an $\alpha$ error probability of 0.05 . An estimated post-exercise hs-cTnT value of $40 \mathrm{ng} / \mathrm{L}$ was based on our previous studies of marathon-induced hs-cTnT release. ${ }^{8,20}$ The expected effect size of $30 \%$ was derived from the literature, where a $40 \%$ reduction of cTn release by RIPC preceding cardiac and vascular surgery has been observed. ${ }^{21-25}$ Assuming a similar effect in exercise-induced cTn release, we felt that a $<30 \%$ reduction would exclude a relevant contribution of ischemia-reperfusion.

Data are presented as mean \pm SEM. A two-factor (time $x$ trial) repeated-measures general model was used to assess difference in biochemical parameters. Additionally, trial order (control or RIPC) was entered into the statistical model as a between-subject variable, partitioning out this source of variability (analyses not shown). In the case of a non-normal distribution, data was transformed by natural logarithm. All statistical tests were two-tailed and a p-value of less than 0.05 was considered statistically significant. All statistical calculations were performed using SPSS version 22 (IBM SPSS Statistics).

\section{Results}

\section{Participants characteristics}

Twenty-nine participants ( 23 males, 6 females) were included in the study. Three male participants left the study prematurely due to a sports-related injury $(n=2)$ or medical reason unrelated to the study protocol $(n=1)$, and were therefore not included in the analysis. One male participant was excluded after study completion because of highly elevated hs-cTnT (Roche), hs-cTnl (Abbott) and sensitive cTnl (s-cTnl, Beckman Coulter) levels (72, 316, and $332 \mathrm{ng} / \mathrm{L}$, respectively) before his second running trial. In the absence of clinical symptoms, these values were highly suggestive for exercise-induced 
cTn release in the past $24 \mathrm{~h}$, and hence indicative for non-compliance to a critical aspect of the study protocol (refrainment of exercise in the $24 \mathrm{~h}$ preceding the run). Therefore, twenty-five participants (19 males, 6 females) with an age of $40 \pm 3$ years (mean \pm SEM) were included in the present analysis (table 1). Subjects were moderately to highly trained, with an average of 52 running km per week (range: 20 - 120). Fourteen runners (56\%) completed at least one marathon or ultra-distance trial. None of the subjects had a history of cardiovascular disease, assessed by medical questionnaire.

Table 1: Participants' characteristics.

\begin{tabular}{ll}
\hline Characteristic & All \\
& $n=25$ \\
\hline Men & $19(76 \%)$ \\
Age, years & $40 \pm 3$ \\
BMI, kg/m² & $22(18-25)$ \\
Physical activity, h/week & $7(2-17)$ \\
Running activity, km/week & $52(20-120)$ \\
Running experience, years & $10(2-30)$ \\
Marathon or ultra-distance experience & $14(56 \%)$ \\
\hline
\end{tabular}

Data are presented as $n(\%)$, mean \pm SEM or mean (range).

\section{Characteristics of the exercise trials}

The exercise trials were separated by a median of 2 weeks (range: 2 - 7). All participants completed both trials within a time span of 1:57:13h - 3:13:36h (h:min:sec), without symptoms of myocardial ischemia. There was no difference in the completion time between the control trial (mean: 2:26:56h) and the trial preceded by RIPC (mean: 2:26:54h). The overall average timeframe between the intervention and the $30 \mathrm{~km}$ run was 24 min (range: 15 - 41) and similar in the setting with and without RIPC. The overall average delay from finish to the first post-exercise blood sample collection was $23 \mathrm{~min}$ (range: 14 - 33) with no differences in the setting with and without RIPC.

\section{Exercise-induced cardiac troponin T peaks earlier than cardiac troponin I}

At baseline of the control trial, almost all athletes displayed hs-cTnT, hs-cTnl and s-cTnl concentrations below the $99^{\text {th }}$ percentile limit ( $n=24,23$ and 24, respectively, figure 1). The completion of a $30 \mathrm{~km}$ run resulted in an expected significant increase of hs-cTnT, hs-cTnl and s-cTnl (time: all $\mathrm{p}<0.001$, figure 1, table 2, supplemental figure 1). Maximum levels of hs-cTnT, hs-cTnl and s-cTnl were $47 \pm 5 \mathrm{ng} / \mathrm{L}, 69 \pm 12 \mathrm{ng} / \mathrm{L}$ and $82 \pm 13$ $\mathrm{ng} / \mathrm{L}$ (mean $\pm \mathrm{SEM}$, control trial) and exceeded the $99^{\text {th }}$ percentile value of hs-cTnT, hscTnl and s-cTnl in 100\% $(n=25), 60 \%(n=15), 68 \%(n=17)$ of the participants. The kinetics of hs-cTnT was different from hs-cTnl and s-cTnl, as $60 \%$ of the participants $(n=$ 
15) displayed hs-cTnT maximum levels at $2 \mathrm{~h}$ post-exercise, whereas maximum values of hs-cTnl and s-cTnl were measured at $5 \mathrm{~h}$ post-exercise in $84 \%$ and $80 \%$ participants ( $n$ $=21$ and $n=20$ ), respectively. Elevations of hs-cTnT, hs-cTnl and s-cTnl measured immediately post-exercise in the control trial were significantly correlated with each other (Pearson R: all $R>0.8, p<0.001$ ). In addition, NTproBNP increased significantly following the $30 \mathrm{~km}$ run with maximum values of $14 \pm 2 \mathrm{pmol} / \mathrm{L}$ (mean $\pm \mathrm{SEM}$, control trial, time: $\mathrm{p}<0.001$, figure 2, table 2, supplemental figure 2). NTproBNP levels were not significantly associated with hs-cTnT, hs-cTnl or s-cTnl (Pearson R: all $\mathrm{R}<0.2, \mathrm{p}>0.3$ ).

Table 2. Release of cardiac and skeletal biomarkers induced by a $30 \mathrm{~km}$ run.

\begin{tabular}{|c|c|c|c|c|c|}
\hline \multirow[t]{2}{*}{ Parameter } & \multirow[t]{2}{*}{ Pre-exercise } & \multicolumn{3}{|c|}{ Post-exercise } & \multirow[t]{2}{*}{$p$-value } \\
\hline & & Oh & $2 \mathrm{~h}$ & $5 h$ & \\
\hline \multicolumn{5}{|l|}{ hs-cTnT, ng/L } & time: $<0.001$ \\
\hline Control & $6 \pm 1$ & $37 \pm 5$ & $45 \pm 5$ & $40 \pm 5$ & trial: 0.2 \\
\hline RIPC & $6 \pm 0$ & $32 \pm 5$ & $42 \pm 6$ & $37 \pm 5$ & time $x$ trial: 0.5 \\
\hline \multicolumn{5}{|l|}{ hs-cTnl, ng/L } & time: $<0.001$ \\
\hline Control & $6 \pm 3$ & $30 \pm 6$ & $49 \pm 8$ & $67 \pm 12$ & trial: 0.4 \\
\hline RIPC & $4 \pm 2$ & $31 \pm 10$ & $53 \pm 16$ & $71 \pm 18$ & time $x$ trial: 0.5 \\
\hline \multicolumn{5}{|l|}{ s-cTnl, ng/L } & time: $<0.001$ \\
\hline Control & $5 \pm 3$ & $37 \pm 7$ & $60 \pm 9$ & $80 \pm 13$ & trial: 0.2 \\
\hline RIPC & $4 \pm 2$ & $33 \pm 11$ & $68 \pm 21$ & $87 \pm 22$ & time $x$ trial: 0.7 \\
\hline \multicolumn{5}{|c|}{ NTproBNP, pmol/L } & time: $<0.001$ \\
\hline Control & $4 \pm 1$ & $14 \pm 2$ & $11 \pm 1$ & $11 \pm 1$ & trial: 0.1 \\
\hline RIPC & $3 \pm 1$ & $11 \pm 1$ & $9 \pm 1$ & $9 \pm 1$ & time $x$ trial: 0.1 \\
\hline \multicolumn{5}{|l|}{$\mathrm{CK}, \mathrm{U} / \mathrm{L}$} & time: $<0.001$ \\
\hline Control & $144 \pm 18$ & $267 \pm 25$ & $314 \pm 25$ & $423 \pm 37$ & trial: 0.9 \\
\hline RIPC & $129 \pm 16$ & $264 \pm 27$ & $323 \pm 33$ & $505 \pm 60$ & time $x$ trial: 0.1 \\
\hline \multicolumn{5}{|l|}{ CK-MB, $\mu \mathrm{g} / \mathrm{L}$} & time: $<0.001$ \\
\hline Control & $4 \pm 1$ & $6 \pm 1$ & $7 \pm 1$ & $10 \pm 1$ & trial: 0.9 \\
\hline RIPC & $4 \pm 0$ & $6 \pm 1$ & $8 \pm 1$ & $12 \pm 1$ & time $x$ trial: 0.2 \\
\hline \multicolumn{5}{|l|}{$\mathrm{LD}, \mathrm{U} / \mathrm{L}$} & time: $<0.001$ \\
\hline Control & $173 \pm 5$ & $274 \pm 12$ & $259 \pm 10$ & $252 \pm 9$ & trial: 0.5 \\
\hline RIPC & $167 \pm 4$ & $276 \pm 13$ & $262 \pm 12$ & $249 \pm 10$ & time $x$ trial: 0.2 \\
\hline \multicolumn{5}{|c|}{$\mathrm{eGFR}, \mathrm{mL} / \mathrm{min} / 1.73 \mathrm{~m}^{2}$} & time: $<0.001$ \\
\hline Control & $110 \pm 2$ & $79 \pm 3$ & $94 \pm 3$ & $103 \pm 3$ & trial: 0.4 \\
\hline RIPC & $108 \pm 2$ & $79 \pm 4$ & $93 \pm 4$ & $100 \pm 4$ & time $x$ trial: 0.6 \\
\hline
\end{tabular}

Data are presented as mean \pm SEM.

NTproBNP, N-terminal pro-B-type natriuretic peptide; CK, creatine kinase; CK-MB, creatine kinase muscle and brain fraction; LD, lactate dehydrogenase; eGFR, estimated glomerular filtration rate. 
A

Figure 1. Release of cardiac troponins induced by a $30 \mathrm{~km}$ run preceded by a control or a remote preconditioning intervention.

Depicted are the overall and individual concentrations of $\mathbf{A}$. hs-cTnT Roche B. hs-cTnl Abbott and C. s-cTnl Beckman Coulter. Overall concentrations are presented as the mean \pm SEM. The dotted line represents the clinical reference value of $14 \mathrm{ng} / \mathrm{L}$ (hs-cTnT), 26.2 $\mathrm{ng} / \mathrm{L}$ (hs-cTnl) and $40 \mathrm{ng} / \mathrm{L}$ (s-cTnl).

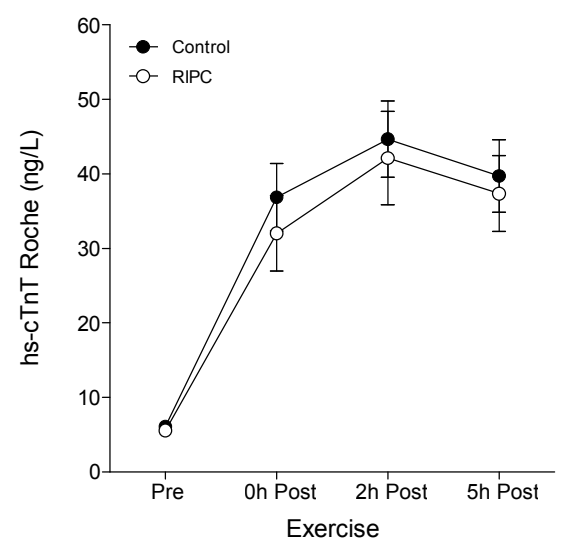

B

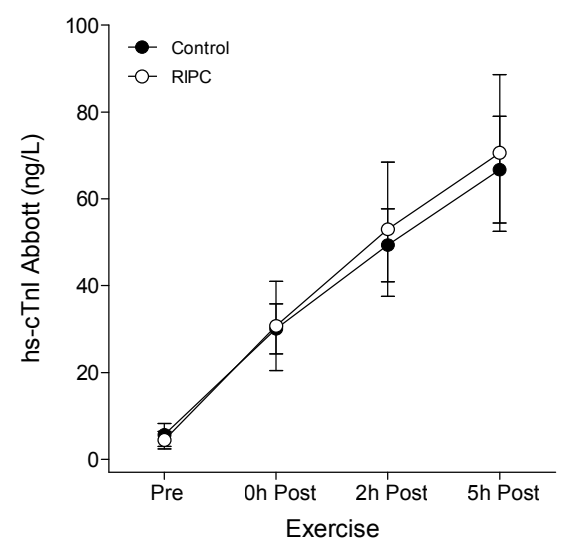

C

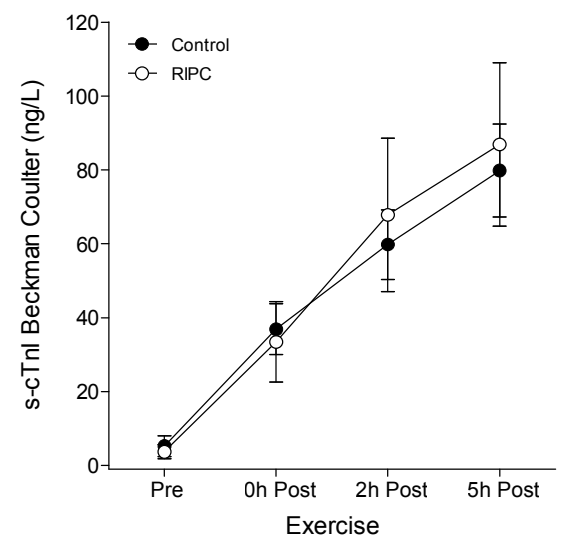


A
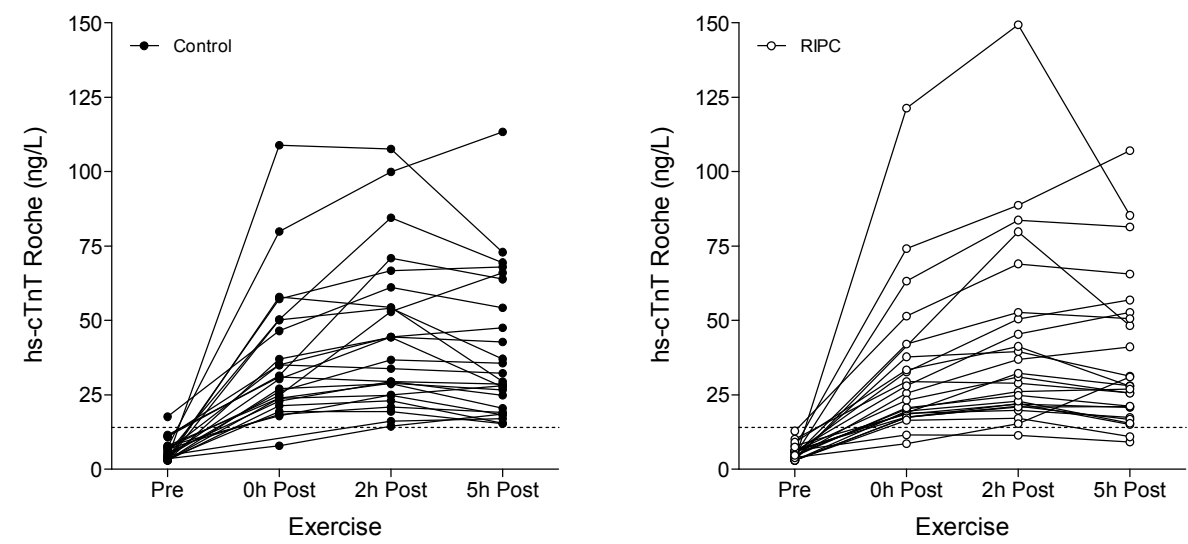

B
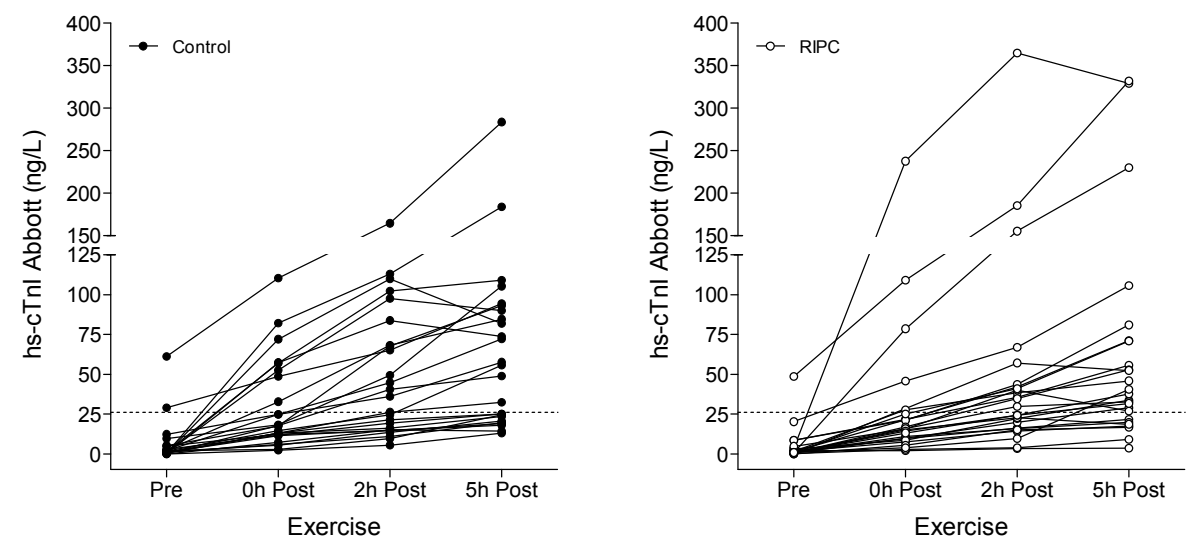

C
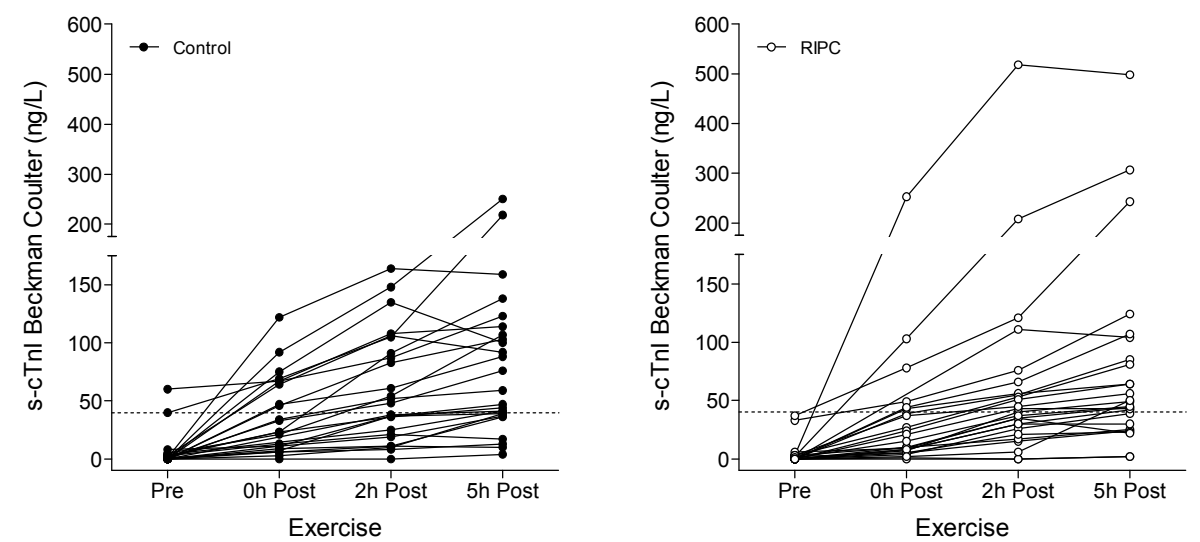
Ischemic preconditioning does not reduce cardiac troponin release

Application of a RIPC stimulus by 4 cycles of $5 \mathrm{~min}$ blood pressure cuff inflation followed by $5 \mathrm{~min}$ of deflation did not reduce exercise-induced cTn release at 0,2 and $5 \mathrm{~h}$ postexercise (figure 1, table 2). Similar results were obtained when correcting for plasma volume changes (supplemental figure 3). The inclusion of trial order and age of the participants in the model did not affect the results (analyses not shown). In addition, exercise-induced changes of NTproBNP, CK, CK-MB, LD and eGFR did not differ between the control and RIPC trial (time $x$ trial: all $p>0.1$, table 2, figure 2).

A

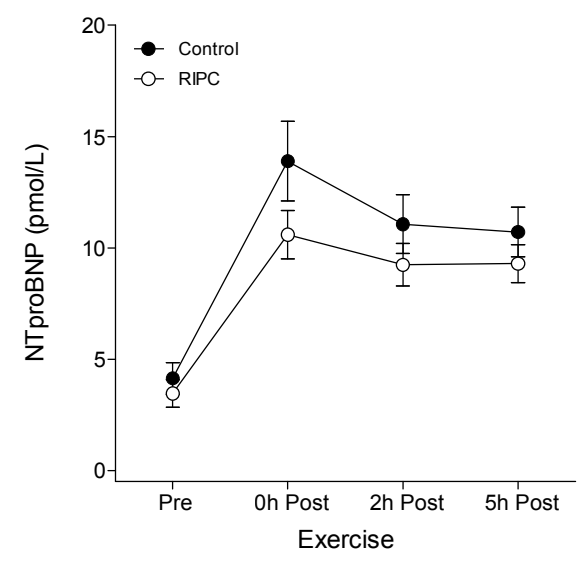

Figure 2. Release of NTproBNP induced by a $30 \mathrm{~km}$ run preceded by a control or remote ischemic preconditioning intervention.

Depicted are the A. overall and B. C. individual concentrations. Overall concentrations are presented as mean \pm SEM.

C
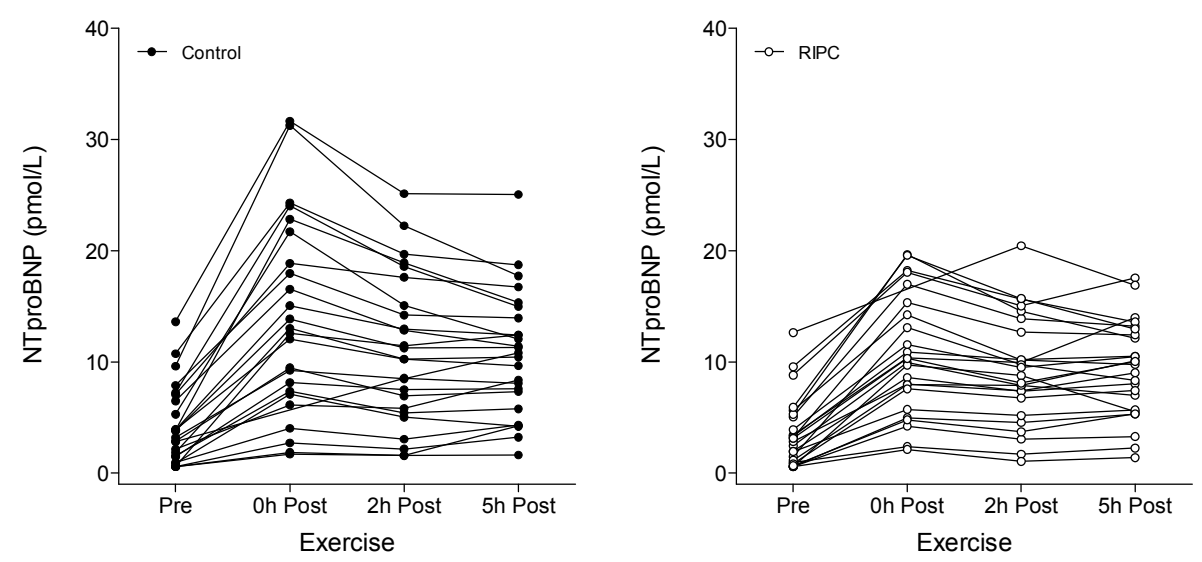


\section{Discussion}

The present study provides a direct comparison of the release kinetics of hs-cTnT and hs-cTnl following prolonged endurance-type exercise. By serial sampling post-exercise, we demonstrate that maximum hs-cTnT levels precede maximum hs-cTnl levels. Furthermore, we show that RIPC, a powerful non-invasive cardio-protective strategy in the setting of ischemia-induced cTn release, has no effect on exercise-induced cTn release.

Recreational running and the participation in long-distance running events, such as (half-)marathons has increased tremendously in recent years. Although the cardiovascular benefits of regular physical exercise are widely established, it is unclear whether this linear dose-benefit response relationship is present among the whole spectrum from regular physical exercise to strenuous (ultra-)endurance-type exercise. ${ }^{26}$ An important issue in this intense scientific debate is the phenomenon of exercise-induced cTn release. This is the first study that measures the release kinetics of both hs-cTnT and hscTnl following prolonged endurance-type exercise. In the majority of the participants, maximum concentrations of hs-cTnT, hs-cTnl and s-cTnl exceeded the clinical reference value. As cTnT and cTnl are biologically equivalent for the diagnosis of $\mathrm{AMI}^{1,27}$ we observed high correlations between all cTn concentrations immediately measured postexercise. However, we demonstrate that hs-cTnT displays maximum concentrations at an earlier stage post-exercise compared to hs-cTnl (2 vs. $>5 \mathrm{~h}$, respectively). This observation is distinct from cTn release resulting from the breakdown of the contractile apparatus following ischemic myocardial injury, where peak levels of hs-cTnT and hs-cTnl are reached within the same time line following hospital admission, followed by cTn elevations for over several days after the onset of myocardial ischemia. ${ }^{13,14}$ Although cTnl and cTnT are different molecules measured with different assays, we observed that median maximum post-exercise hs-cTnl levels were numerically in the same range of hs-cTnT levels (69 $\mathrm{ng} / \mathrm{L}$ and $47 \mathrm{ng} / \mathrm{L}$, respectively). Remarkably, these concentrations are distinct from cTn release in AMI patients, where hs-cTnl values have been measured in multiples of hs-cTnT (range of multiple of hs-cTnl levels compared to hs-cTnT: 3.4 12.4). ${ }^{13,28,29}$ All together, these observations suggest that exercise-induced cTn release may be mechanistically distinct from irreversible cardiomyocyte injury in AMI.

An aim of this study was to assess the effect of RIPC on exercise-induced cardiac biomarker release. This cardio-protective strategy has been successfully applied to pathological settings, where cTn release results from ischemia-reperfusion injury induced by an intervention. Application of RIPC in for example cardiac surgery or angioplasty has been shown to significantly reduce post-intervention cTn release, which further translates into an improvement in all-cause mortality. ${ }^{15,16,30,31}$ The exact signaling cascade of RIPC is the subject of intense research, but it is evident that the mediator 
triggers protection ubiquitously in all visceral organs, by the activation of cryoprotective signaling. ${ }^{32,33}$ We observed no effect of a RIPC stimulus preceding an endurance-type exercise trial on the kinetics of hs-cTnT, hs-cTnl, s-cTnl and other cardiac or skeletal biomarkers. By the time this study was conducted, a paper was published where also no effect of RIPC on hs-cTnl levels induced by a cycling exercise trial was observed. ${ }^{34}$ However, no direct comparison of cTnT and cTnl kinetics was performed. In the present study, we applied running as the exercise model and measured both hscTnT and hs-cTnl kinetics. Taken together, these findings favor a mechanism of cTn release unrelated to ischemia in the setting of endurance-type exercise.

The biological mechanism underlying exercise-induced cTn release remains unclear and there is currently limited evidence for a specific mechanism. Several other theories involving the altered environment of cardiomyocytes have been suggested, such as stimulation of integrins related to myocardial stretch or the formation of free oxygen radicals. Similar to findings in the literature, we observed no significant correlation between NTproBNP concentrations immediately post-exercise and hs-cTnT, hs-cTnl or $\mathrm{s}-\mathrm{cTnl},{ }^{11,35}$ which makes the contribution of myocardial stretch in exercise-induced cTn release unlikely. In addition, the role of free oxygen radicals as contributing mechanism is lacking due to the observations that antioxidant supplementation had no effect on exercise-induced cTn release. ${ }^{36,37}$

Although our acute findings of endurance-type exercise suggest that the underlying mechanism may be different from irreversible cardiomyocyte injury in AMI, findings of subclinical coronary artery disease, right ventricular (RV) fibrotic lesions and RV ar-

rhythmias have been observed among extensively trained (veteran) athletes. ${ }^{38-41}$ The susceptibility of individual athletes to these injurious effects of strenuous exercise is not fully understood and the role of exercise-induced hs-cTnT and hs-cTnl kinetics in the risk-stratification of individual athletes is unclear.

To conclude, exercise-induced cTn release is a universal phenomenon where maximum hs-cTnT levels are reached at an earlier stage than maximum hs-cTnl levels. Moreover, the application of a RIPC stimulus did not reduce exercise-induced CTn release, which favors a mechanism of cTn unrelated to ischemia in the setting of endurance-type exercise.

\section{Acknowledgements}

The authors gratefully acknowledge the assistance of Martijn Bischoff and Vincent Kleijnen. This study was supported by a research grant from Stichting De Weijerhorst to M.v.D.-V. Abbott Diagnostics and Beckman Coulter provided troponin I assays to M.v.D.V. and S.M. 


\section{References}

1. Thygesen K, Alpert JS, Jaffe AS, et al. Third universal definition of myocardial infarction. Circulation. 2012;126(16):2020-2035.

2. Giannitsis E, Katus HA. Cardiac troponin level elevations not related to acute coronary syndromes. Nat Rev Cardiol. 2013;10(11):623-634.

3. Mohlenkamp S, Leineweber K, Lehmann N, et al. Coronary atherosclerosis burden, but not transient troponin elevation, predicts long-term outcome in recreational marathon runners. Basic Res Cardiol. 2014;109(1):391.

4. Whyte GP. Clinical significance of cardiac damage and changes in function after exercise. Med Sci Sports Exerc. 2008;40(8):1416-1423.

5. Shave R, Baggish A, George K, et al. Exercise-induced cardiac troponin elevation: evidence, mechanisms, and implications. J Am Coll Cardiol. 2010;56(3):169-176.

6. Klinkenberg $\amalg$, Res PT, van Loon LJ, van Dieijen-Visser MP, Meex SJ. Strong link between basal and exercise-induced cardiac troponin T levels: do both reflect risk? Int J Cardiol. 2012;158(1):129-131.

7. Eijsvogels T, George K, Shave R, et al. Effect of prolonged walking on cardiac troponin levels. Am J Cardiol. 2010;105(2):267-272.

8. Mingels A, Jacobs L, Michielsen E, Swaanenburg J, Wodzig W, van Dieijen-Visser M. Reference population and marathon runner sera assessed by highly sensitive cardiac troponin $\mathrm{T}$ and commercial cardiac troponin T and I assays. Clin Chem. 2009;55(1):101-108.

9. Neumayr G, Pfister R, Mitterbauer G, Eibl G, Hoertnagl H. Effect of competitive marathon cycling on plasma $\mathrm{N}$-terminal pro-brain natriuretic peptide and cardiac troponin $\mathrm{T}$ in healthy recreational cyclists. Am J Cardiol. 2005;96(5):732-735.

10. Tian Y, Nie J, Huang C, George KP. The kinetics of highly sensitive cardiac troponin T release after prolonged treadmill exercise in adolescent and adult athletes. J Appl Physiol (1985). 2012;113(3):418425.

11. Scherr J, Braun S, Schuster T, et al. 72-h kinetics of high-sensitive troponin T and inflammatory markers after marathon. Med Sci Sports Exerc. 2011;43(10):1819-1827.

12. Morrow DA, Cannon CP, Jesse RL, et al. National Academy of Clinical Biochemistry Laboratory Medicine Practice Guidelines: clinical characteristics and utilization of biochemical markers in acute coronary syndromes. Clin Chem. 2007;53(4):552-574.

13. Laugaudin G, Kuster N, Petiton A, et al. Kinetics of high-sensitivity cardiac troponin $T$ and I differ in patients with ST-segment elevation myocardial infarction treated by primary coronary intervention. Eur Heart J Acute Cardiovasc Care. 2015.

14. Solecki K, Dupuy AM, Kuster N, et al. Kinetics of high-sensitivity cardiac troponin $\mathrm{T}$ or troponin I compared to creatine kinase in patients with revascularized acute myocardial infarction. Clin Chem Lab Med. 2015;53(5):707-714.

15. Kharbanda RK, Nielsen TT, Redington AN. Translation of remote ischaemic preconditioning into clinical practice. Lancet. 2009;374(9700):1557-1565.

16. Kharbanda RK, Mortensen UM, White PA, et al. Transient limb ischemia induces remote ischemic preconditioning in vivo. Circulation. 2002;106(23):2881-2883.

17. Jean-St-Michel E, Manlhiot C, Li J, et al. Remote preconditioning improves maximal performance in highly trained athletes. Med Sci Sports Exerc. 2011;43(7):1280-1286.

18. Giannitsis E, Kurz K, Hallermayer K, Jarausch J, Jaffe AS, Katus HA. Analytical validation of a highsensitivity cardiac troponin T assay. Clin Chem. 2010;56(2):254-261.

19. Inker LA, Schmid $\mathrm{CH}$, Tighiouart $\mathrm{H}$, et al. Estimating glomerular filtration rate from serum creatinine and cystatin C. N Engl J Med. 2012;367(1):20-29.

20. Mingels AM, Jacobs LH, Kleijnen VW, et al. Cardiac troponin T elevations, using highly sensitive assay, in recreational running depend on running distance. Clin Res Cardiol. 2010;99(6):385-391.

21. Ali ZA, Callaghan CJ, Lim E, et al. Remote ischemic preconditioning reduces myocardial and renal injury after elective abdominal aortic aneurysm repair: a randomized controlled trial. Circulation. 2007;116(11 Suppl):|98-105. 
22. Hausenloy DJ, Mwamure PK, Venugopal $\mathrm{V}$, et al. Effect of remote ischaemic preconditioning on myocardial injury in patients undergoing coronary artery bypass graft surgery: a randomised controlled trial. Lancet. 2007;370(9587):575-579.

23. Cheung MM, Kharbanda RK, Konstantinov IE, et al. Randomized controlled trial of the effects of remote ischemic preconditioning on children undergoing cardiac surgery: first clinical application in humans. J Am Coll Cardiol. 2006;47(11):2277-2282.

24. Hoole SP, Heck PM, Sharples L, et al. Cardiac Remote Ischemic Preconditioning in Coronary Stenting (CRISP Stent) Study: a prospective, randomized control trial. Circulation. 2009;119(6):820-827.

25. Venugopal V, Hausenloy DJ, Ludman A, et al. Remote ischaemic preconditioning reduces myocardial injury in patients undergoing cardiac surgery with cold-blood cardioplegia: a randomised controlled trial. Heart. 2009;95(19):1567-1571.

26. La Gerche A, Heidbuchel H. Can intensive exercise harm the heart? You can get too much of a good thing. Circulation. 2014;130(12):992-1002.

27. Rubini Gimenez M, Twerenbold R, Reichlin T, et al. Direct comparison of high-sensitivity cardiac troponin I vs. T for the early diagnosis of acute myocardial infarction. Eur Heart J. 2014;35(34):2303-2311.

28. Rubini Gimenez M, Hoeller R, Reichlin T, et al. Rapid rule out of acute myocardial infarction using undetectable levels of high-sensitivity cardiac troponin. Int J Cardiol. 2013;168(4):3896-3901.

29. Lindahl B, Eggers KM, Venge $P$, James $S$. Evaluation of four sensitive troponin assays for risk assessment in acute coronary syndromes using a new clinically oriented approach for comparison of assays. Clin Chem Lab Med. 2013;51(9):1859-1864.

30. Thielmann $M$, Kottenberg $E$, Kleinbongard $P$, et al. Cardioprotective and prognostic effects of remote ischaemic preconditioning in patients undergoing coronary artery bypass surgery: a single-centre randomised, double-blind, controlled trial. Lancet. 2013;382(9892):597-604.

31. Sloth AD, Schmidt MR, Munk K, et al. Improved long-term clinical outcomes in patients with ST-elevation myocardial infarction undergoing remote ischaemic conditioning as an adjunct to primary percutaneous coronary intervention. Eur Heart J. 2014;35(3):168-175.

32. Hausenloy DJ, Yellon DM. Remote ischaemic preconditioning: underlying mechanisms and clinical application. Cardiovasc Res. 2008;79(3):377-386.

33. Bell R, Yellon D. Surgery: Remote ischaemic conditioning-approaching prime time? Nat Rev Cardiol. 2013;10(11):619-621.

34. El Messaoudi S, Vissers A, Thijssen D, Riksen NP, Rongen GA. The effect of remote ischemic preconditioning on exercise-induced plasma troponin I appearance in healthy volunteers. Int J Cardiol. 2013;168(2):1612-1613.

35. Salvagno GL, Schena F, Gelati M, et al. The concentration of high-sensitivity troponin I, galectin-3 and NT-proBNP substantially increase after a 60-km ultramarathon. Clin Chem Lab Med. 2014;52(2):267272.

36. Klinkenberg LJ, Res PT, Haenen GR, et al. Effect of antioxidant supplementation on exercise-induced cardiac troponin release in cyclists: a randomized trial. PLoS One. 2013;8(11):e79280.

37. Howatson G, Goodall S, Hill J, et al. Antioxidant supplementation does not attenuate exercise-induced cardiac troponin release. Int J Cardiol. 2011;152(1):101-102.

38. Mohlenkamp S, Lehmann N, Breuckmann F, et al. Running: the risk of coronary events : Prevalence and prognostic relevance of coronary atherosclerosis in marathon runners. Eur Heart J. 2008;29(15):19031910.

39. Baldesberger $\mathrm{S}$, Bauersfeld $\mathrm{U}$, Candinas $\mathrm{R}$, et al. Sinus node disease and arrhythmias in the long-term follow-up of former professional cyclists. Eur Heart J. 2008;29(1):71-78.

40. Breuckmann F, Mohlenkamp S, Nassenstein K, et al. Myocardial late gadolinium enhancement: prevalence, pattern, and prognostic relevance in marathon runners. Radiology. 2009;251(1):50-57.

41. Abdulla J, Nielsen JR. Is the risk of atrial fibrillation higher in athletes than in the general population? A systematic review and meta-analysis. Europace. 2009;11(9):1156-1159.

42. Dill DB, Costill DL. Calculation of percentage changes in volumes of blood, plasma, and red cells in dehydration. J Appl Physiol. 1974;37(2):247-248. 


\section{Supplemental information}

\section{Supplemental figures}

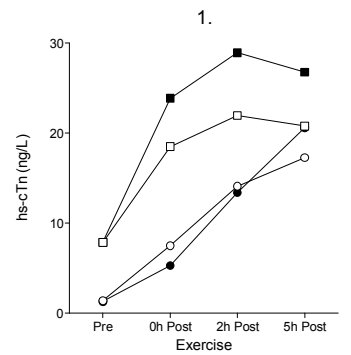

4.

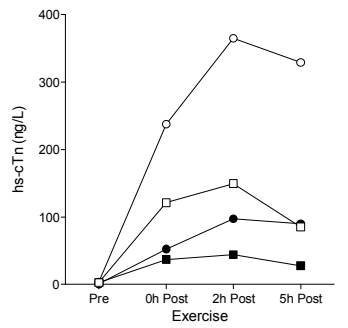

7.

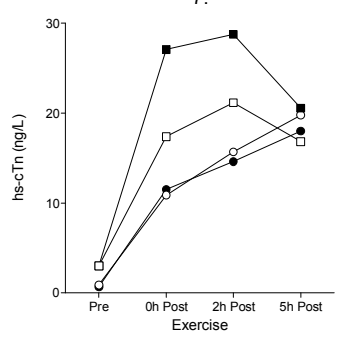

10.

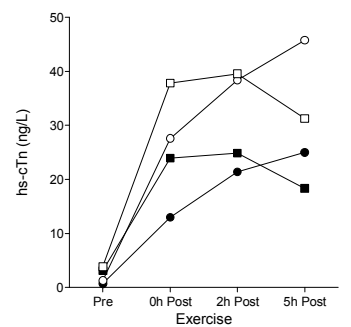

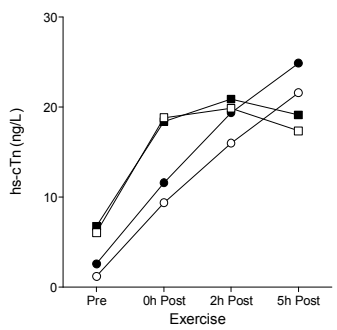

5.

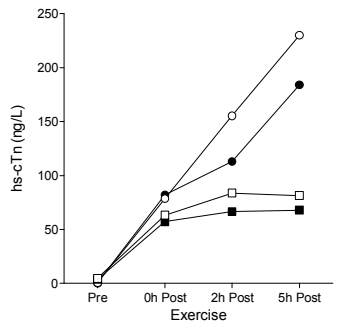

8.

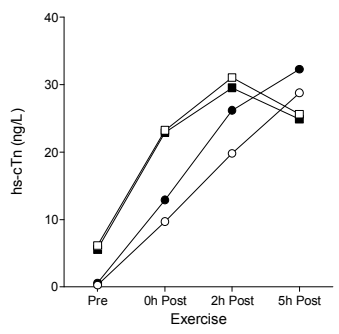

11.

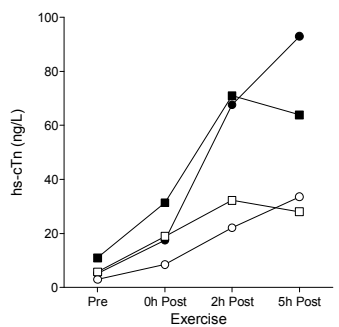

3.

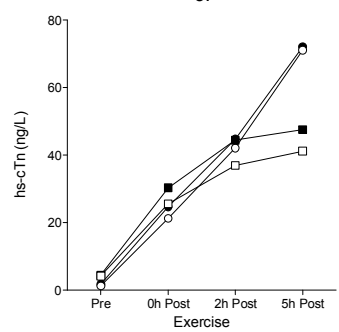

6.

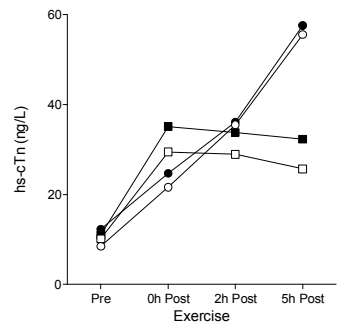

9.

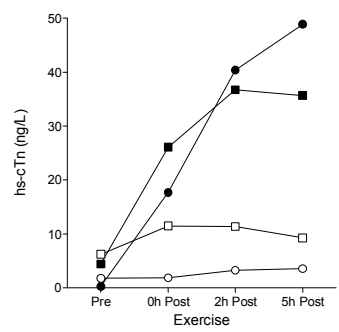

12.

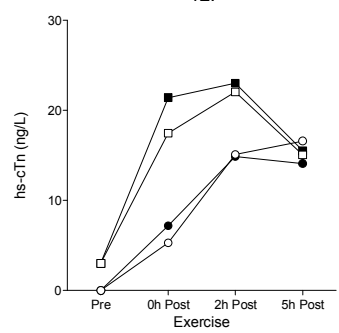

Supplemental figure 1. Individual release of hs-cTnl and hs-cTnT induced by a $30 \mathrm{~m}$ run preceded by a control or remote ischemic preconditioning intervention (continued on next page).

The numbering is identical to supplemental figure 2, allowing direct comparisons between figures.
hs-cTnl Control
hs-cTnl RIPC

as-cTnT Control

$\square$ hs-cTnT RIPC 
13.

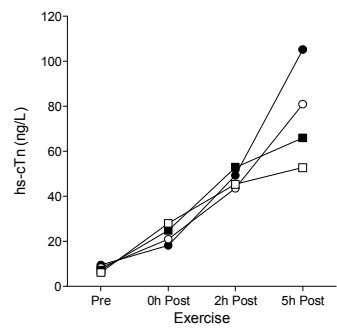

16.

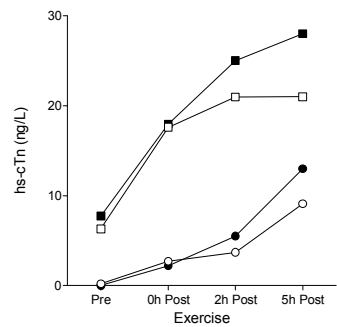

19.

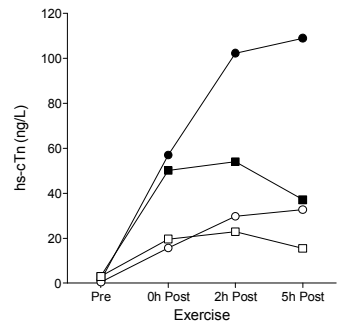

22.

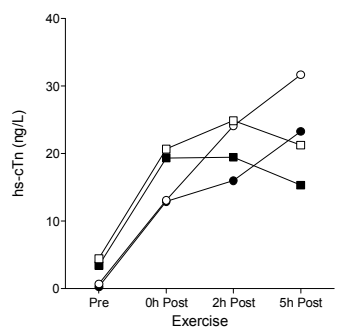

25

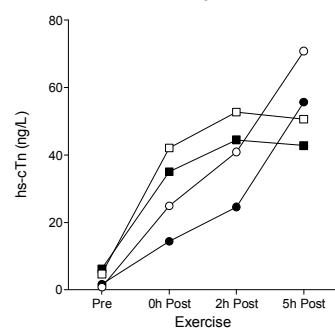

14.

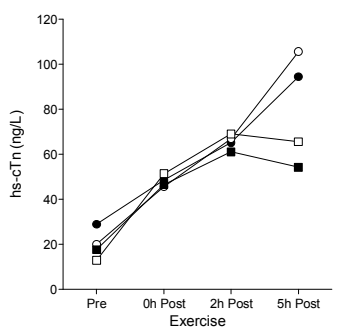

17.

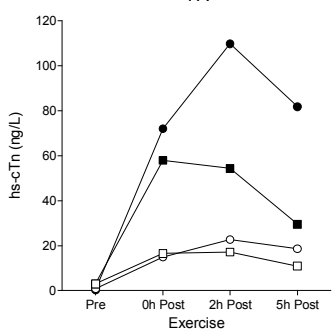

20.

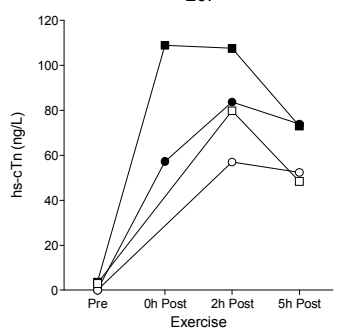

23

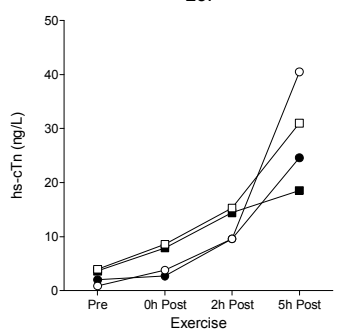

15.

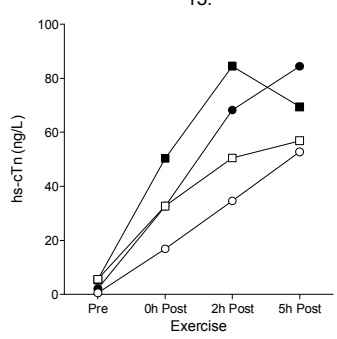

18.

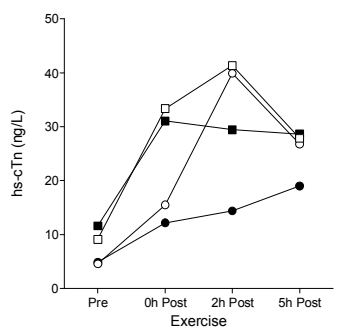

21.

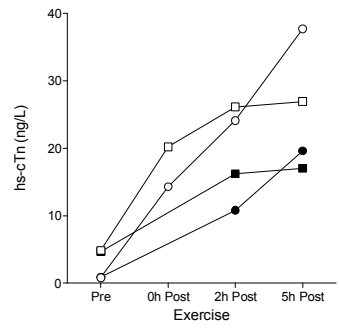

24.

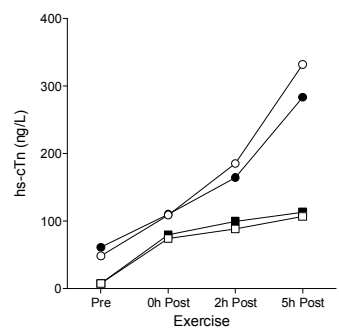

Supplemental figure 1. Individual release of hs-cTnl and hs-cTnT induced by a $30 \mathrm{~km}$ run preceded by a control or remote ischemic preconditioning intervention (continued on previous page).

The numbering is identical to supplemental figure 2, allowing direct comparisons between figures.

- hs-cTnl Control O hs-cTnI RIPC

ahs-cTnT Control $\square$ hs-cTnT RIPC 
1.

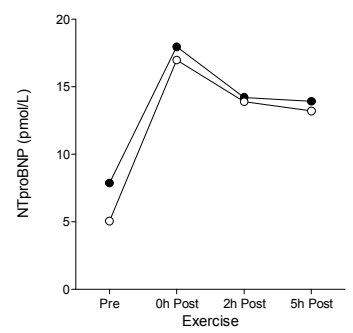

4

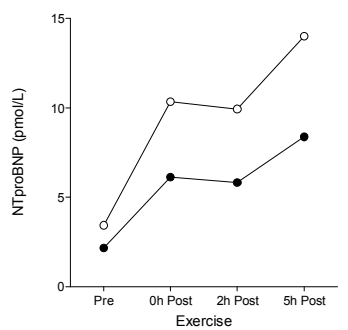

7.

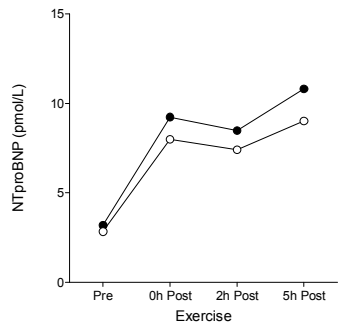

10.

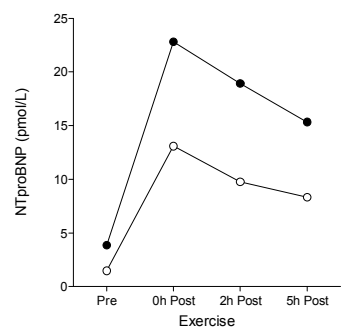

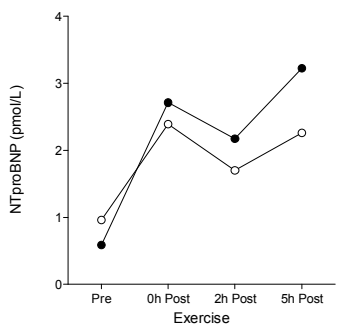

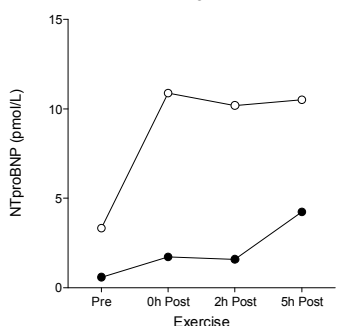

8.

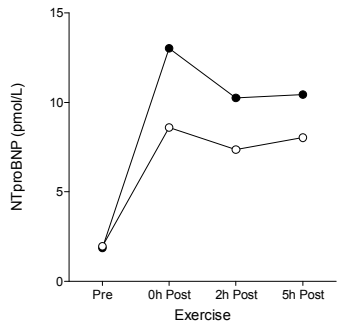

11.

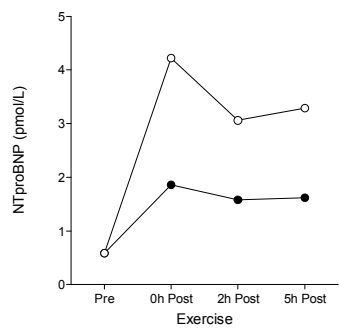

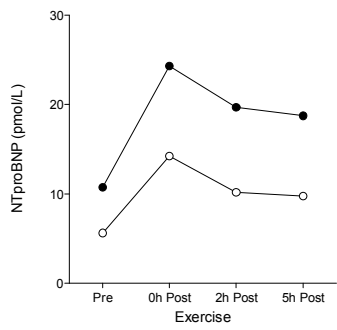

6

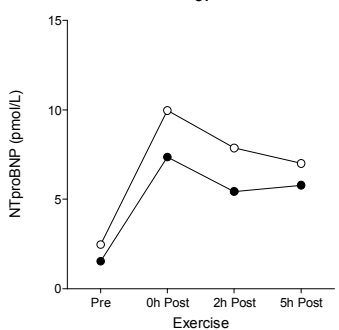

9.

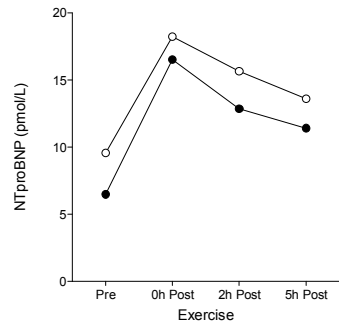

12.

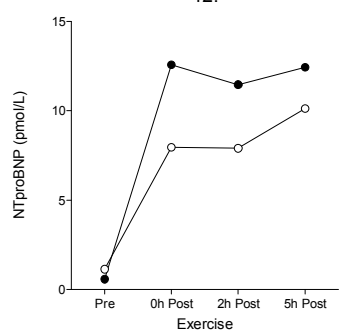

Supplemental figure 2. Individual release of NTproBNP induced by a $30 \mathrm{~km}$ run preceded by a control or remote ischemic preconditioning intervention (continued on next page).

The numbering is identical to supplemental figure 1, allowing direct comparisons between figures.
- Control
O RIPC 
13.

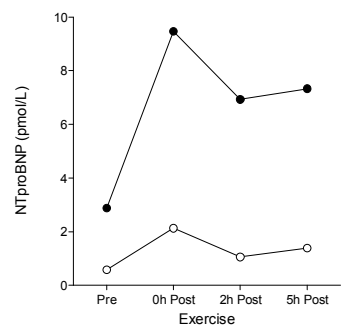

16.

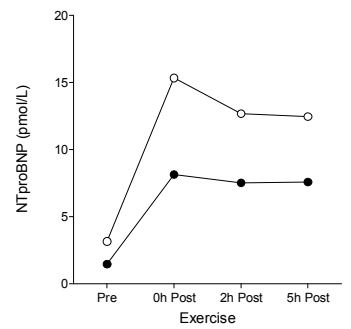

19.

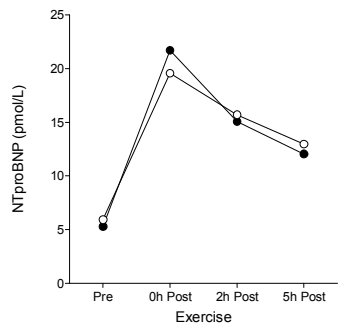

22.

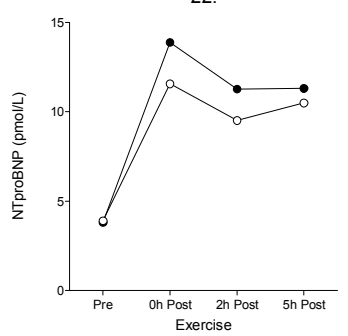

25.

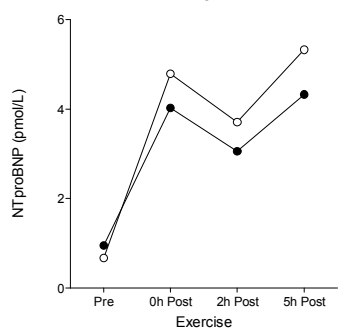

14.

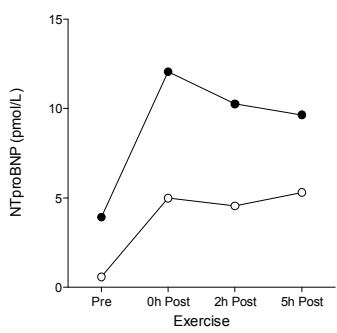

17.

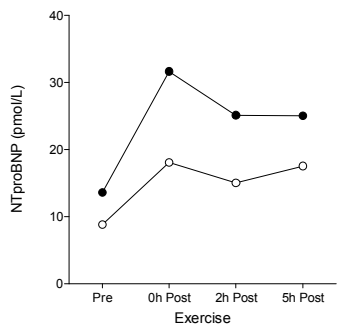

20.

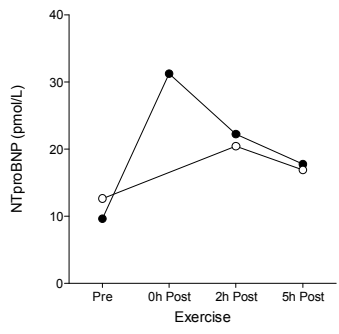

23.

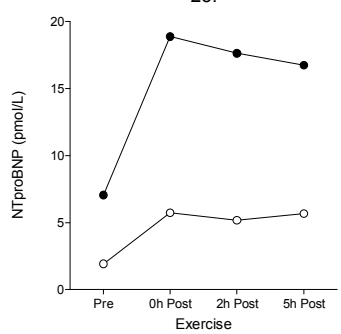

15.

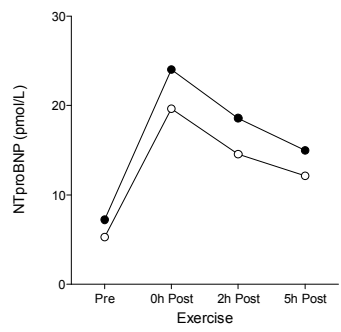

18.

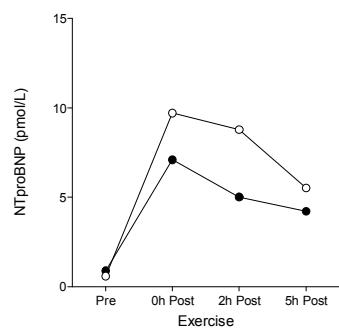

21.

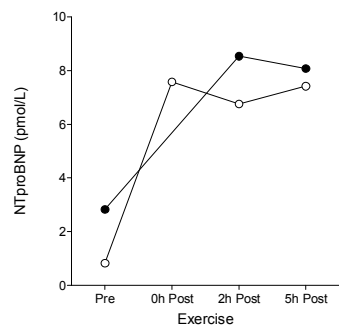

24.

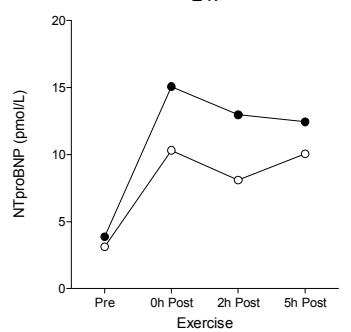

Supplemental figure 2. Individual release of NTproBNP induced by a 30 $\mathrm{km}$ run preceded by a control or remote ischemic preconditioning intervention (continued on next page).

The numbering is identical to supplemental figure 1, allowing direct comparisons between figures.

- Control O RIPC 
A

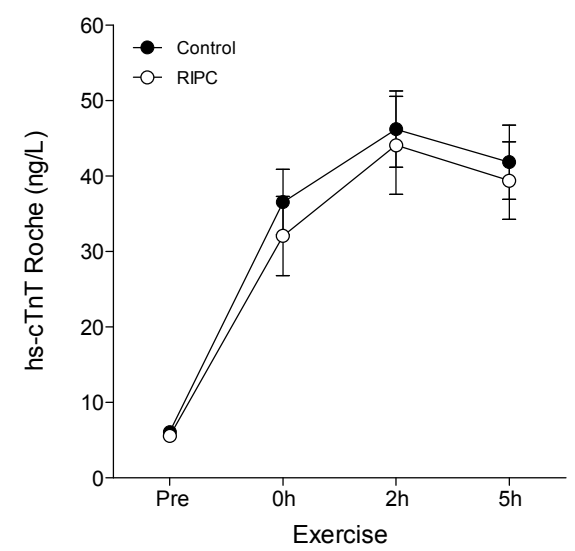

C

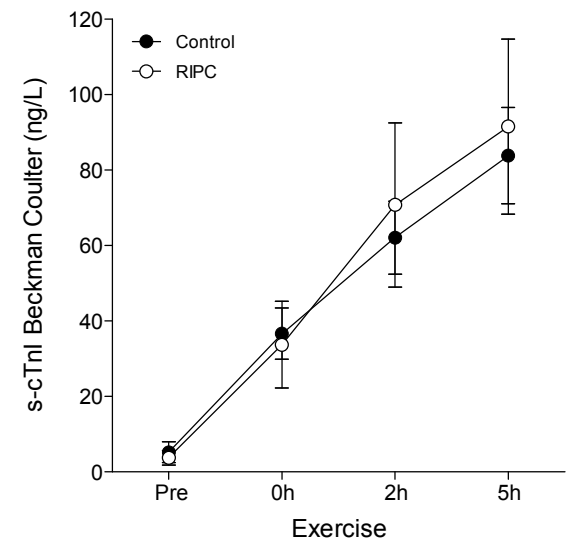

B

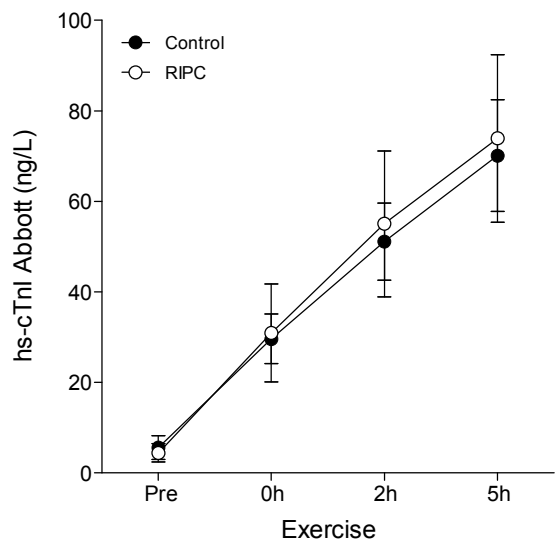

D

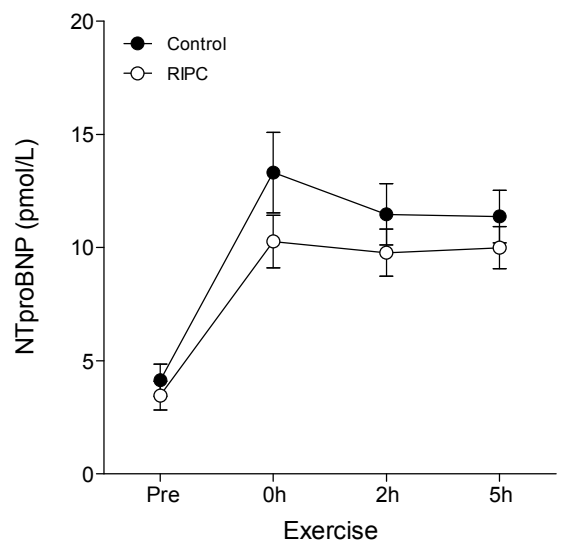

Supplemental figure 3. Release of cardiac biomarkers induced by a $30 \mathrm{~km}$ run preceded by a control or remote ischemic preconditioning intervention.

Concentrations of A. hs-cTnT Roche B. hs-cTnl Abbott C. s-cTnl Beckman Coulter D. NTproBNP were corrected according to the change in plasma volume on the basis of pre-exercise plasma volume status. ${ }^{42}$ 


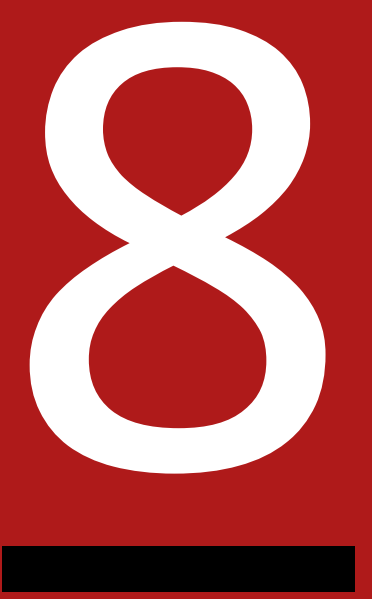


General discussion 
The diagnosis of acute myocardial infarction (AMI) relies strongly on the release of cardiac troponins (cTn) (either cardiac troponin I [cTnl] or T [cTnT]) into the bloodstream, which are the preferred biomarkers of myocardial injury. ${ }^{1-4} \mathrm{cTnl}$ and $\mathrm{cTnT}$ are subunits of the troponin complex with distinct characteristics in terms of their molecular mass (cTnl: $23 \mathrm{kDa}, \mathrm{cTnT}: 35 \mathrm{kDa}$ ) and their function in the regulation of muscle contraction. ${ }^{5,6}$ Nevertheless, cTnl and cTnT are diagnostically equivalent for the detection of cardiomyocyte injury according to the universal definition of AMI. ${ }^{1}$ A recent survey among European laboratories demonstrated that $50 \%$ of the laboratories measure cTnT and $45 \%$ use cTnl as the preferred biomarker for the clinical diagnosis of suspected AMI. ${ }^{7}$ Recent advancements in the analytical performance of troponin assays have resulted in more sensitive and accurate detection of circulating cTn. ${ }^{8,9}$ One of the first high-sensitivity (hs) assays was the hs-cTnT assay of Roche Diagnostics. Therefore, the initial studies that validated the hs-cTnT assay concluded (not completely unexpected) that hs-cTnT was diagnostically and prognostically superior to cTnl measured with contemporary sensitive assays. ${ }^{10-12}$ To date, the (clinical) availability of highsensitivity assays for both $\mathrm{cTnl}$ and $\mathrm{cTnT}$, provides the methodology to directly compare the performance of $\mathrm{cTnl}$ and $\mathrm{cTnT}$ in various clinical and experimental settings (this thesis).

\section{Serial testing of cardiac troponins}

\section{Delta criteria for the interpretation of serial cardiac troponins}

The introduction of high-sensitivity assays for cTn has created both challenges and opportunities with regard to the diagnostic workup for AMI. ${ }^{13}$ A major opportunity is the improvement in the early diagnosis of AMI through an increased diagnostic sensitivity. ${ }^{10,11}$ A major challenge is the finding that cTn are not exclusively released into the bloodstream as a consequence of ischemic cardiomyocyte injury, ${ }^{14}$ but also during numerous acute and chronic conditions, such as pulmonary embolism, type 2 diabetes mellitus and chronic kidney disease. ${ }^{15-17}$

To improve the specificity of hs-cTn for AMI, the diagnostic workup of AMI relies on serial testing and the assessment of kinetic changes in hs-cTn. ${ }^{1-4}$ A critical assumption when interpreting kinetic changes is that serial measurements of hs-cTn - in clinically stable conditions - display true random variation around an individual's homeostatic set point. Based on this assumption, two broad categories of studies have aimed to quantify the 'delta' cTn value over a specified period that best discriminates between acute and chronic elevations of cTn. The first category comprised biological variation studies in healthy individuals to calculate the reference change value (RCV) from analytical and biological variation, to distinguish 'true changes' from 'noise' or random physiological 
fluctuations. For example, studies in healthy individuals reported short term ( 1 - 4h) RCVs ranging from $11-85 \% .{ }^{18-21}$ The second category of studies employed a data-driven approach where patterns of cTn concentrations in suspected AMI patients were associated with their adjudicated clinical diagnosis. These studies have demonstrated an improvement in overall diagnostic accuracy for AMI by incorporating a delta criterion at 2 - $6 \mathrm{~h}^{22-26}$ for example an absolute delta change criteria of $7 \mathrm{ng} / \mathrm{L}$ in hs-cTnT within a $2 \mathrm{~h}$ period improved the diagnostic area under the curve from $95 \%$ to $98 \%{ }^{24}$ It should be recognized, however, that there is no perfect diagnostic threshold and the application of any delta criteria thus far is a trade-off, favoring greater specificity at the expense of sensitivity with higher delta criteria. ${ }^{27,28}$ Despite the lack of a delta threshold recommendation for a specified period and assay in the guidelines, the American College of Cardiology / American Heart Association Task Force on Practice Guidelines recommends a minimum of $20 \%$ change as a rule of thumb. ${ }^{2,27}$

\section{Diurnal variation and diagnosis of acute myocardial infarction}

It is inevitably clear that interpretation of both the magnitude and the pattern of cTn elevation in the overall clinical context is essential in the diagnostic workup of AMI, which is based on the assumption of random variation in serial measurements in individuals without acute cardiovascular disease. Chapters 2 and 3 demonstrate that this critical assumption is not valid in case for hs-cTnT. These chapters shows that hs-cTnT exhibits a diurnal rhythm, characterized by gradually decreasing values throughout daytime, rising concentrations during nighttime, to peak concentrations in the morning. In addition, chapter 2 demonstrates that violation of the assumption of random variation has direct consequences for the calculation of RCVs. Due to the diurnal rhythm of hs-cTnT, the calculated RCV strongly depends on the time frame chosen. In a period of 'high-level changes' (8:30 A.M. - 12:30 P.M.) the RCV is more than twice the RCV in a period of 'low-level changes' (4:30 P.M. - 8:30 P.M.). Chapter 3 further illustrates that diurnal oscillation is only restricted to hs-cTnT, with random fluctuations of cTnl during day and night assessed with two (hs-)cTnl assays.

The diurnal fluctuation of hs-cTnT in some individuals exceeded the suggested deltachange criteria of $20 \%$ for AMI. $^{2}$ Therefore in conjunction with typical clinical symptoms of myocardial ischemia, these physiological fluctuations have the potential to be interpreted as biochemical evidence of myocardial injury. Accordingly, the clinical effect of the diurnal rhythmic oscillation on the diagnostic accuracy for AMI was further evaluated in chapter 3. This chapter demonstrates that characteristics of the diurnal hs-cTnT rhythm persist in real-life chest pain patients with an adjudicated diagnosis of noncardiac disease, by significantly higher hs-cTnT levels in patients presenting in the earlymorning hours compared to patients presenting to the cardiac emergency department in the evening. The overall effect of the diurnal rhythm on the diagnostic accuracy of hs- 
cTnT for AMI is fortunately rather small. Identical to hs-cTnl, the diagnostic accuracy of hs-cTnT for AMl at presentation, $1 \mathrm{~h}$ and the combination of absolute changes with presenting concentration is very high and independent on the timeframe of patient presentation to the cardiac emergency department. Nevertheless, the effect of the timeframe of patient presentation is more pronounced and statistically significant, in patients presenting early after the onset of chest pain. In patients presenting in the evening, where the physiological rhythm displays minor fluctuations, absolute changes of hs-cTnT within $1 \mathrm{~h}$ have a significant higher diagnostic accuracy compared to patients presenting in the early-morning, where the diurnal hs-cTnT rhythm reaches its 'highlevel phase'. Therefore, the presence of a diurnal rhythm may provide an explanation in isolated cases of patients where the clinical assessment is discordant with biochemical hs-cTnT kinetics. Consequently, the physiological rise and fall of hs-cTnT during day and night is an important novel finding for clinicians.

\section{Diurnal variation in cardiovascular physiology and pathology}

The rhythmicity of hs-cTnT is in line with the diurnal organization of the cardiovascular system. ${ }^{29,30}$ Diurnal rhythmicity with peak activity in the (early) morning hours has been described for many cardiovascular traits: heart rate, sympathetic activity, vascular resistance, the renin-angiotensin aldosterone system, prothrombotic tendency, platelet aggregability and the fibrinolytic system. ${ }^{31-35}$ The rhythmicity of these traits is reflected in the circadian variation of the incidence and clinical significance of cardiovascular events. For example, the highest incidence of AMI is found in the morning hours, in addition to the largest infarct sizes and higher mortality rates that are observed among patients with AMI that commence in the morning hours. ${ }^{36-38}$

\section{Release and clearance rates of cardiac troponins}

Circulating hs-cTnl and hs-cTnT concentrations are defined by their release and clearance rates. In order to understand the diurnal hs-cTnT rhythm, it is essential to gain insight in the mechanism that underlies chronic cTn release. Proposed mechanisms include cardiomyocyte cell death, physiological cell turn over, or transient cTn leakage through membranous bleb formation, but all mechanisms currently lack sufficient confirmatory data. ${ }^{14,39-40}$ Currently, there are no conclusive data demonstrating differences between cTnl and cTnT in terms of their cytosolic or cellular content, or their initial release upon cardiomyocyte injury. Regardless of the underlying mechanism of chronic cTn release, elevated hs-cTn are strongly associated with adverse outcomes, irrespective of the underlying disease. ${ }^{14,41}$

Apart from the release of cTn, various studies demonstrate that hs-cTnT concentrations are more susceptible to changes in kidney function than hs-cTnl. First, a greater proportion of patients with chronic kidney disease has elevated CTnT or hs-cTnT con- 
centrations above the diagnostic cut-off for AMI relative to cTnl or hs-cTnl. ${ }^{17,42,43}$ Furthermore, the estimated glomerular filtration rate (eGFR) is stronger associated with hsCTnT than hs-cTnl in patients with chronic kidney disease with or without symptoms of AMI. ${ }^{17,44}$ This stronger association of hs-cTnT and eGFR compared to hs-cTnl has been confirmed in patients with stable coronary artery disease. ${ }^{45}$ These differences suggests that different half-lives of cTnT and cTnl may underlie - at least in part - differences in rhythmic oscillation. Further studies are required to investigate whether conditions that influence the half-lives of cTnT and cTnl will affect the amplitude of hs-cTnT oscillation. For example, by hourly sampling patients with chronic kidney disease (with eGFR of 30 $59 \mathrm{~mL} / \mathrm{min} / 1.73 \mathrm{~m}^{2}$ and $15-29 \mathrm{~mL} / \mathrm{min} / 1.73 \mathrm{~m}^{2}$ ) in a total time span of $25 \mathrm{~h}$.

\section{Cardiac troponin I and T}

\section{Absolute concentrations in acute and chronic settings}

In chapter 3, hs-cTnl and hs-cTnT were measured in a setting of chronic cTn release. Although cTnl and cTnT are different molecules, and measured with different assays, we observed that the median hs-cTnT concentration at 8:30 A.M. was numerically higher than the hs-cTnl level ( $15.8 \mathrm{ng} / \mathrm{L}$ and $6.5 \mathrm{ng} / \mathrm{L}$, respectively). These findings are supported by peer-reviewed literature that described higher mean or median values of hs-cTnT compared to hs-cTnl (either measured with the Abbott assay, prototype Siemens assay or prototype Beckman Coulter assay) measured in chronic settings of cTn release (stable coronary artery disease, chronic kidney disease, dialysis (table 1). ${ }^{17,18,46-49}$ On the other hand, in studies describing acute release of cTn by both hs-cTnl and hs- cTnT caused by AMI, median or mean hs-cTnl levels at presentation to the cardiac emergency department were higher than hs-cTnT values. ${ }^{12,50-52}$ This overview indicates that acute release of cTn might be characterized by higher hs-cTnl levels compared to hs-cTnT, compared to chronic release of cTn where lower hs-cTnl levels relative to hs-cTnT concentrations might be observed.

\section{Prognostic value of cardiac troponins}

It is inevitably clear that elevated levels of hs-cTn are associated with an increased risk of developing cardiovascular events and all-cause or cardiovascular mortality, regardless of an acute of chronic setting of cTn release. ${ }^{16,17,45,53,54}$ In addition, even in the general population and when adjusted for traditional risk factors, hs-cTn are important predictors for an adverse outcome. ${ }^{55-58}$ Nevertheless, the prognostic value of hs-cTn in apparently healthy subjects from the general population has not been systematically assessed and possible differences in the prognostic performance of cTnl and CTnT are unexplored. 


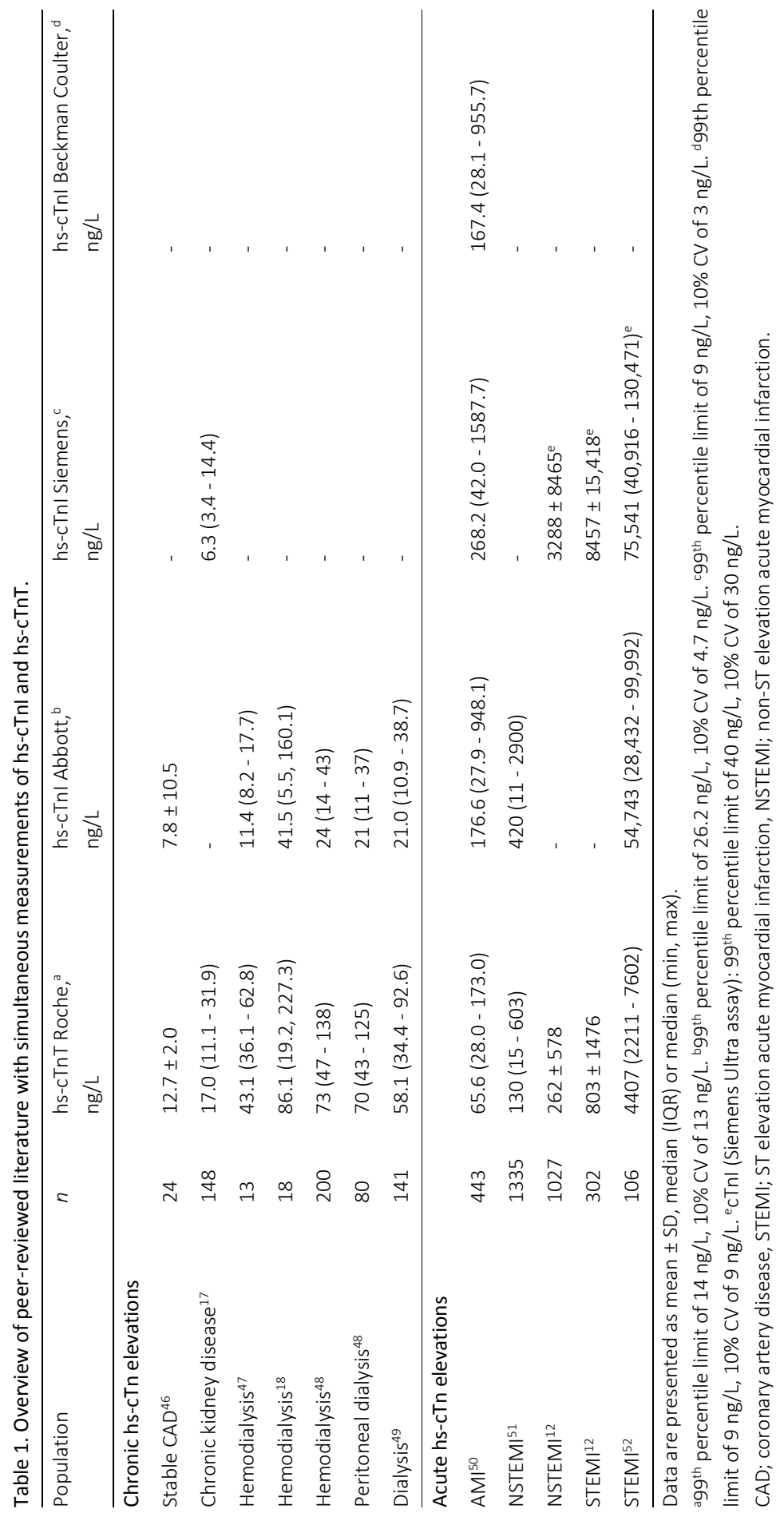


Chapter 4 demonstrates by a meta-regression analysis of nine studies a trend towards a better prognostic value of basal levels of hs-cTnT compared to hs-cTnl in the general population. Similarly, CTnT was found to be a better predictor for all-cause mortality in direct comparisons of the prognostic value of (hs-)cTnT and (hs-)cTnl in patient populations such as acute chest pain, AMI, heart failure, end-stage renal disease and dialysis. ${ }^{12,43,49,53,59,60}$ Nevertheless, a recent study demonstrated that hs-cTnl and hscTnT provide additional prognostic information in patients with stable coronary artery disease. ${ }^{45}$ Future studies are therefore essential to investigate whether the prognostic differences between hs-cTnl and hs-cTnT are clinically relevant, and whether both biomarkers provide independent and additional prognostic value.

\section{Exercise-induced cardiac troponins release}

Prolonged endurance-type exercise is accompanied by the release of cTn into the circulation. ${ }^{61}$ To date, the mechanism of cTn release during and after endurance-type exercise is unclear. The aim of the chapters 5, 6 and 7 was to study the heterogeneity in cTn release within and between individuals and the underlying mechanism of exerciseinduced cTn release. In this section, these results will be coupled to epidemiological, biomarker, functional and electrophysiological studies of the heart in endurance-trained (recreational) athletes to provide insight in the underlying nature of exercise-induced cTn release.

\section{Clinical significance of exercise-induced cardiac troponins release}

The cardiovascular benefits of regular physical exercise are widely established. Individuals exercising regularly have a favorable cardiovascular risk profile for the development of coronary artery disease, partly mediated through an improved insulin sensitivity, serum lipid levels, and blood pressure control. ${ }^{62-64}$ The detection of circulating cTn after endurance-type exercise, with elevations often exceeding the diagnostic cut-off, is therefore a counter-intuitive phenomenon. In this thesis, three findings were reported that (indirectly) suggest that exercise-induced cTn release may be mechanistically distinct from acute release of cTn in AMI patients. First, chapters 5, 6, and 7 demonstrate that an increase in circulating cTn occurs in almost every individual after a cycling or running trial. Second, chapter 5 demonstrates that exercise-induced cTn release is a reproducible phenomenon with a very strong correlation of post-exercise levels during two identical standardized cycling trials. Third, by serial sampling of healthy athletes post-exercise, chapter 7 demonstrates that hs-cTnT levels peaks earlier than hs-cTnl levels, which is different from the release kinetics in AMI, where initial peak levels are reached at similar hours post-admission. ${ }^{52,65}$ In contrast to acute and chronic cTn re- 
lease in cardiac and non-cardiac disease, ${ }^{14,41}$ a recent study demonstrated that exerciseinduced cTn release is not related to long-term cardiovascular outcome. ${ }^{66}$ All together, these findings suggest that cTn release following endurance-type exercise may be a physiological process, i.e. reversible cardiomyocyte damage.

\section{Mechanisms of exercise-induced cardiac troponins release}

Although the kinetics of cTn release observed after endurance-type exercise suggest a mechanism unrelated to irreversible cardiomyocyte damage, several aspects of exercise-induced cTn release require further study. The biological mechanism underlying exercise-induced cTn release remains unclear. Supplementation of astaxanthin, a powerful antioxidant, described in chapter 6 had no effect on elevations of hs-cTnT observed after a cycling trial. This study was based on the hypothesis that oxidative stress associated with prolonged exercise may contribute to exercise-induced cTn release and that antioxidant supplementation has been shown to attenuate oxidative stress, inflammation and muscle indices following endurance-type exercise. ${ }^{67,68}$ Chapter 6 describes that astaxanthin does not improve antioxidant capacity, nor has an effect on exercise-induced cTn release, despite substantial increases of plasma astaxanthin concentrations. In addition, the application of a remote ischemic preconditioning (RIPC) stimulus had no effect on the kinetics of hs-cTnl and hs-cTnT induced by a $30 \mathrm{~km}$ running trial, as evaluated in chapter 7. RIPC is a powerful non-invasive cardio-protective strategy, whereby brief episodes of non-lethal ischemia followed by reperfusion of one tissue or organ confers protection to other organs against an injurious ischemic insult. ${ }^{69}$ This cardio-protective strategy has been successfully applied in pathological settings characterized by irreversible cardiomyocyte damage. ${ }^{70}$ Application of RIPC in for example cardiac surgery or angioplasty has been shown to significantly reduce postintervention cTn release, which further translates to an improvement in all-cause mortality. ${ }^{71,72}$ The lack of an effect of RIPC on exercise-induced cTn release favors a mechanism of cTn release unrelated to ischemia in the setting of endurance-type exercise.

It has been suggested that a small proportion of cTn (3-8\%) exists as functionally free protein in the cytosolic compartment. ${ }^{73-75}$ The initial release of cTn in AMI possibly reflects the release of the cytosolic cTn pool, followed by a second prolonged elevation that results from the breakdown of the contractile apparatus. It has been hypothesized that cTn release is a continuum from reversible injury towards irreversible injury. ${ }^{76}$ Rapid rise and fall of cTn in the first hours after exercise may therefore be consistent with the release of the cytosolic pool (reversible cardiomyocyte injury) rather than irreversible cardiomyocyte injury where a time-dependent fall over a longer period is expected because of gradual degradation of myofibrils and release of the troponin complex. ${ }^{76}$ A recent study demonstrated in vitro that the cytosolic CTnT component is probably not a fixed percentage, but dependent on a reversible binding with tropomyo- 
$\sin ^{77}$ This possible early releasable pool in the cytosol following reversible cardiomyocyte injury requires further investigation.

Endurance-type exercise training stimulates physiological cardiac adaptations that facilitate appropriate increases in cardiac output during exercise. For example, endurance-trained individuals exhibit mostly eccentric hypertrophy, which is characterized by dilatation of the cardiac chambers and an increase in the maximal wall thickness. ${ }^{78-80}$ The inverse association of training status with post-exercise concentrations of cTn, ${ }^{81,82}$ as well as an increase in baseline and post-exercise cTn after an endurance training program, ${ }^{83}$ suggest that elevations of cTn may be linked to structural and functional cardiac adaptations in response to endurance-type exercise. However, the relation between biochemical and functional cardiac changes following endurance-type exercise is currently unclear.

\section{Endurance-type exercise: can the heart have too much?}

The cardiovascular benefits of exercise are achieved by relatively modest doses of exercise, for example 5 to 10 min running per day at slow speeds $(<10 \mathrm{~km} / \mathrm{h})$ or 15 min brisk walking per day. ${ }^{84,85}$ The intensity of training programs of endurance athletes are 5 - 10 times higher than the workload of these exercise recommendations as a preventive measure for coronary artery disease. ${ }^{86}$ Such intense levels of exercise require a combination of electrical, structural and functional cardiac adaptations, termed the athlete's heart, to generate a sustained 5 - 6 fold increased cardiac output. ${ }^{87}$ Recent studies suggest that too much exercise could be harmful, as demonstrated by the presence of subclinical right ventricular (RV) myocardial fibrosis in a minority of athletes and a higher prevalence or RV arrhythmia's in ultra-endurance athletes compared to sedentary controls. ${ }^{88-94}$ These striking observations are the basis of the current hypothesis that there is an upper limit to the healthy athlete's heart. This concept states that ultraendurance exercise results in RV short-term dysfunction, ${ }^{95}$ which in some studies has been shown to correlate to post-exercise elevations of cTn. ${ }^{93,96}$ When the RV dysfunction is more severe by a too intense training, or without adequate recovery between ultra-endurance exercise bouts, the over-training of the heart may cause cardiac injury and may result in the development of pro-arrhythmic remodeling. ${ }^{86,97}$ Therefore, further studies conducting a detailed and longitudinal assessment of large groups of ultraendurance athletes incorporating cardiovascular magnetic resonance, echocardiographic and biomarker measurements is necessary to evaluate the impact of a lifetime of endurance-type exercise on the heart. 


\section{Concluding remarks and future directions}

The advancements in analytical performance of the troponin assays has expanded the role of cardiac troponins (cTn) from diagnostics to prognostics, and may broaden its clinical utility from acute myocardial infarction (AMI) to a broad spectrum of cardiac and non-cardiac diseases. A novel finding of this thesis is the presence of a diurnal rhythm of hs-cTnT in individuals with no acute cardiovascular disease. This is an important finding for clinicians, as patients with chest pain but without a diagnosis of AMI display characteristics of the physiological rise and fall of hs-cTnT during day and night. It is to date unclear whether the clearance of circulating hs-cTnT levels by the kidneys contributes to the diurnal oscillation. Although the diurnal rhythm of hs-cTnT has no effect on the overall diagnostic accuracy for AMI, it may impact the diagnostic accuracy in specified patient subgroups, for example in patients presenting (very) early after the onset of chest pain, or in patients with chronic kidney disease. These findings stress the importance of interpreting dynamic changes of cTn in relation to the clinical presentation.

Furthermore, this thesis described differences between hs-cTnT and hs-cTnl concentrations in terms of their diurnal variation and prognostic value. Future studies should aim to compare hs-cTnT and hs-cTnl and evaluate whether both biomarkers provide independent and additional diagnostic or prognostic value across the broad spectrum of cardiovascular disease.

Finally, this thesis demonstrated that exercise-induced cTn release is a reproducible phenomenon characterized by an earlier peak of hs-cTnT post-exercise compared to hscTnl. In addition, the absence of an effect by antioxidant supplementation and remote ischemic preconditioning, argues against oxidative stress and an imbalance in oxygen supply-demand as major contributors to the release of cTn in the setting of endurancetype exercise. 


\section{References}

1. Thygesen K, Alpert JS, Jaffe AS, et al. Third universal definition of myocardial infarction. Eur Heart J. 2012;33(20):2551-2567.

2. Amsterdam EA, Wenger NK, Brindis RG, et al. 2014 AHA/ACC Guideline for the Management of Patients with Non-ST-Elevation Acute Coronary Syndromes: a report of the American College of Cardiology/American Heart Association Task Force on Practice Guidelines. J Am Coll Cardiol. 2014;64(24):e139-228.

3. Hamm CW, Bassand JP, Agewall S, et al. ESC Guidelines for the management of acute coronary syndromes in patients presenting without persistent ST-segment elevation: The Task Force for the management of acute coronary syndromes (ACS) in patients presenting without persistent ST-segment elevation of the European Society of Cardiology (ESC). Eur Heart J. 2011;32(23):2999-3054.

4. Thygesen K, Mair J, Giannitsis E, et al. How to use high-sensitivity cardiac troponins in acute cardiac care. Eur Heart J. 2012;33(18):2252-2257.

5. Thygesen K, Mair J, Katus $\mathrm{H}$, et al. Recommendations for the use of cardiac troponin measurement in acute cardiac care. Eur Heart J. 2010;31(18):2197-2204.

6. Katrukha IA. Human cardiac troponin complex. Structure and functions. Biochemistry (Mosc). 2013;78(13):1447-1465.

7. Collinson PO, van Dieijen-Visser MP, Pulkki K, et al. Evidence-based laboratory medicine: how well do laboratories follow recommendations and guidelines? The Cardiac Marker Guideline Uptake in Europe (CARMAGUE) study. Clin Chem. 2012;58(1):305-306.

8. de Lemos JA. Increasingly sensitive assays for cardiac troponins: a review. JAMA. 2013;309(21):22622269.

9. Apple FS, Collinson PO. Analytical characteristics of high-sensitivity cardiac troponin assays. Clin Chem. 2012;58(1):54-61.

10. Reichlin T, Hochholzer W, Bassetti S, et al. Early diagnosis of myocardial infarction with sensitive cardiac troponin assays. N Engl J Med. 2009;361(9):858-867.

11. Keller $\mathrm{T}$, Zeller $\mathrm{T}$, Peetz $\mathrm{D}$, et al. Sensitive troponin I assay in early diagnosis of acute myocardial infarction. N Engl J Med. 2009;361(9):868-877.

12. Mueller M, Celik S, Biener $\mathrm{M}$, et al. Diagnostic and prognostic performance of a novel high-sensitivity cardiac troponin T assay compared to a contemporary sensitive cardiac troponin I assay in patients with acute coronary syndrome. Clin Res Cardiol. 2012;101(10):837-845.

13. Mueller M, Vafaie M, Biener M, Giannitsis E, Katus HA. Cardiac troponin T: from diagnosis of myocardial infarction to cardiovascular risk prediction. Circ J. 2013;77(7):1653-1661.

14. Giannitsis E, Katus HA. Cardiac troponin level elevations not related to acute coronary syndromes. Nat Rev Cardiol. 2013;10(11):623-634.

15. Muller-Bardorff M, Weidtmann B, Giannitsis E, Kurowski V, Katus HA. Release kinetics of cardiac troponin T in survivors of confirmed severe pulmonary embolism. Clin Chem. 2002;48(4):673-675.

16. Everett BM, Cook NR, Magnone MC, et al. Sensitive cardiac troponin T assay and the risk of incident cardiovascular disease in women with and without diabetes mellitus: the Women's Health Study. Circulation. 2011;123(24):2811-2818.

17. deFilippi C, Seliger SL, Kelley W, et al. Interpreting cardiac troponin results from high-sensitivity assays in chronic kidney disease without acute coronary syndrome. Clin Chem. 2012;58(9):1342-1351.

18. Aakre KM, Roraas T, Petersen $\mathrm{PH}$, et al. Weekly and 90-minute biological variations in cardiac troponin $\mathrm{T}$ and cardiac troponin I in hemodialysis patients and healthy controls. Clin Chem. 2014;60(6):838-847.

19. Frankenstein L, Wu AH, Hallermayer K, Wians FH, Jr., Giannitsis E, Katus HA. Biological variation and reference change value of high-sensitivity troponin $T$ in healthy individuals during short and intermediate follow-up periods. Clin Chem. 2011;57(7):1068-1071.

20. Vasile VC, Saenger AK, Kroning JM, Klee GG, Jaffe AS. Biologic variation of a novel cardiac troponin I assay. Clin Chem. 2011;57(7):1080-1081.

21. Dupuy AM, Lozano C, Badiou S, Bargnoux AS, Kuster N, Cristol JP. Biological variability of hs-cardiac troponin T on the Roche Cobas 8000/e602(R) immunoanalyzer. Clin Chim Acta. 2013;425:62-63. 
22. Apple FS, Pearce LA, Smith SW, Kaczmarek JM, Murakami MM. Role of monitoring changes in sensitive cardiac troponin I assay results for early diagnosis of myocardial infarction and prediction of risk of adverse events. Clin Chem. 2009;55(5):930-937.

23. Keller T, Zeller T, Ojeda F, et al. Serial changes in highly sensitive troponin I assay and early diagnosis of myocardial infarction. JAMA. 2011;306(24):2684-2693.

24. Reichlin T, Irfan A, Twerenbold R, et al. Utility of absolute and relative changes in cardiac troponin concentrations in the early diagnosis of acute myocardial infarction. Circulation. 2011;124(2):136-145.

25. Mueller $\mathrm{M}$, Biener $\mathrm{M}$, Vafaie $\mathrm{M}$, et al. Absolute and relative kinetic changes of high-sensitivity cardiac troponin $T$ in acute coronary syndrome and in patients with increased troponin in the absence of acute coronary syndrome. Clin Chem. 2012;58(1):209-218.

26. Cullen L, Parsonage WA, Greenslade J, et al. Delta troponin for the early diagnosis of AMI in emergency patients with chest pain. Int J Cardiol. 2013;168(3):2602-2608.

27. Morrow DA, Bonaca MP. Real-world application of "delta" troponin: diagnostic and prognostic implications. J Am Coll Cardiol. 2013;62(14):1239-1241.

28. Bjurman C, Larsson M, Johanson $\mathrm{P}$, et al. Small changes in troponin T levels are common in patients with non-ST-segment elevation myocardial infarction and are linked to higher mortality. J Am Coll Cardiol. 2013;62(14):1231-1238.

29. Martino TA, Sole MJ. Molecular time: an often overlooked dimension to cardiovascular disease. Circ Res. 2009;105(11):1047-1061.

30. Sato M, Matsuo T, Atmore H, Akashi M. Possible contribution of chronobiology to cardiovascular health. Front Physiol. 2013;4:409.

31. Mancia G, Ferrari A, Gregorini L, et al. Blood pressure and heart rate variabilities in normotensive and hypertensive human beings. Circ Res. 1983;53(1):96-104.

32. Williams GH, Cain JP, Dluhy RG, Underwood RH. Studies of the control of plasma aldosterone concentration in normal man. I. Response to posture, acute and chronic volume depletion, and sodium loading. J Clin Invest. 1972;51(7):1731-1742.

33. Tofler GH, Brezinski D, Schafer Al, et al. Concurrent morning increase in platelet aggregability and the risk of myocardial infarction and sudden cardiac death. N Engl J Med. 1987;316(24):1514-1518.

34. Fearnley GR, Balmforth G, Fearnley E. Evidence of a diurnal fibrinolytic rhythm; with a simple method of measuring natural fibrinolysis. Clin Sci (Lond). 1957;16(4):645-650.

35. Panza JA, Epstein SE, Quyyumi AA. Circadian variation in vascular tone and its relation to alphasympathetic vasoconstrictor activity. N Engl J Med. 1991;325(14):986-990.

36. Muller JE, Stone PH, Turi ZG, et al. Circadian variation in the frequency of onset of acute myocardial infarction. N Engl J Med. 1985;313(21):1315-1322.

37. Reiter R, Swingen C, Moore L, Henry TD, Traverse JH. Circadian dependence of infarct size and left ventricular function after ST elevation myocardial infarction. Circ Res. 2012;110(1):105-110.

38. Manfredini R, Boari B, Bressan S, et al. Influence of circadian rhythm on mortality after myocardial infarction: data from a prospective cohort of emergency calls. Am J Emerg Med. 2004;22(7):555-559.

39. Bergmann O, Bhardwaj RD, Bernard S, et al. Evidence for cardiomyocyte renewal in humans. Science. 2009;324(5923):98-102.

40. Hickman PE, Potter JM, Aroney C, et al. Cardiac troponin may be released by ischemia alone, without necrosis. Clin Chim Acta. 2010;411(5-6):318-323.

41. Agewall S, Giannitsis E, Jernberg T, Katus H. Troponin elevation in coronary vs. non-coronary disease. Eur Heart J. 2011;32(4):404-411.

42. Jacobs LH, van de Kerkhof J, Mingels AM, et al. Haemodialysis patients longitudinally assessed by highly sensitive cardiac troponin $\mathrm{T}$ and commercial cardiac troponin $\mathrm{T}$ and cardiac troponin I assays. Ann Clin Biochem. 2009;46(Pt 4):283-290.

43. Apple FS, Murakami MM, Pearce LA, Herzog CA. Predictive value of cardiac troponin I and T for subsequent death in end-stage renal disease. Circulation. 2002;106(23):2941-2945.

44. Twerenbold R, Wildi K, Jaeger C, et al. Optimal Cutoff Levels of More Sensitive Cardiac Troponin Assays for the Early Diagnosis of Myocardial Infarction in Patients With Renal Dysfunction. Circulation. 2015;131(23):2041-2050.

45. Omland T, Pfeffer MA, Solomon SD, et al. Prognostic value of cardiac troponin I measured with a highly sensitive assay in patients with stable coronary artery disease. J Am Coll Cardiol. 2013;61(12):12401249. 
46. Nordenskjold AM, Ahlstrom H, Eggers KM, et al. Short- and long-term individual variation in cardiac troponin in patients with stable coronary artery disease. Clin Chem. 2013;59(2):401-409.

47. Cardinaels EP, Cornelis T, van der Sande FM, et al. Acute effects of conventional and extended hemodialysis and hemodiafiltration on high-sensitivity cardiac troponins. Clin Chem Lab Med. 2015;53(11)e289-e292.

48. Snaedal S, Bárány P, Querishi AR, et al. High sensitive troponin T and troponin I three month variation in prevalent hemodialysis and peritoneal dialysis patients. Nephrology Dialysis Transplantation. 2015;30 (Supplement 3):iii556-iii569.

49. Hickman PE, McGill D, Potter JM, Koerbin G, Apple FS, Talaulikar G. Multiple biomarkers including cardiac troponins $T$ and I measured by high-sensitivity assays, as predictors of long-term mortality in patients with chronic renal failure who underwent dialysis. Am J Cardiol. 2015;115(11):1601-1606.

50. Rubini Gimenez M, Hoeller R, Reichlin T, et al. Rapid rule out of acute myocardial infarction using undetectable levels of high-sensitivity cardiac troponin. Int J Cardiol. 2013;168(4):3896-3901.

51. Lindahl B, Eggers KM, Venge $P$, James S. Evaluation of four sensitive troponin assays for risk assessment in acute coronary syndromes using a new clinically oriented approach for comparison of assays. Clin Chem Lab Med. 2013;51(9):1859-1864.

52. Laugaudin G, Kuster N, Petiton A, et al. Kinetics of high-sensitivity cardiac troponin $T$ and I differ in patients with ST-segment elevation myocardial infarction treated by primary coronary intervention. Eur Heart J Acute Cardiovasc Care. 2015.

53. Haaf $P$, Reichlin $T$, Twerenbold $R$, et al. Risk stratification in patients with acute chest pain using three high-sensitivity cardiac troponin assays. Eur Heart J. 2014;35(6):365-375.

54. van Wijk S, Jacobs L, Eurlings LW, et al. Troponin T measurements by high-sensitivity vs conventional assays for risk stratification in acute dyspnea. Clin Chem. 2012;58(1):284-292.

55. deFilippi CR, de Lemos JA, Christenson RH, et al. Association of serial measures of cardiac troponin T using a sensitive assay with incident heart failure and cardiovascular mortality in older adults. JAMA. 2010;304(22):2494-2502.

56. Saunders JT, Nambi V, de Lemos JA, et al. Cardiac troponin T measured by a highly sensitive assay predicts coronary heart disease, heart failure, and mortality in the Atherosclerosis Risk in Communities Study. Circulation. 2011;123(13):1367-1376.

57. Gore MO, Seliger SL, Defilippi CR, et al. Age- and sex-dependent upper reference limits for the highsensitivity cardiac troponin T assay. J Am Coll Cardiol. 2014;63(14):1441-1448.

58. Eggers KM, Lind L, Venge $P$, Lindahl B. Factors influencing the 99th percentile of cardiac troponin I evaluated in community-dwelling individuals at 70 and 75 years of age. Clin Chem. 2013;59(7):10681073.

59. Rubini Gimenez M, Twerenbold R, Reichlin T, et al. Direct comparison of high-sensitivity cardiac troponin I vs. T for the early diagnosis of acute myocardial infarction. Eur Heart J. 2014;35(34):2303-2311.

60. de Antonio M, Lupon J, Galan A, et al. Head-to-head comparison of high-sensitivity troponin $T$ and sensitive-contemporary troponin । regarding heart failure risk stratification. Clin Chim Acta. 2013;426:18-24.

61. Shave R, Baggish A, George K, et al. Exercise-induced cardiac troponin elevation: evidence, mechanisms, and implications. J Am Coll Cardiol. 2010;56(3):169-176.

62. Tanasescu M, Leitzmann MF, Rimm EB, Willett WC, Stampfer MJ, Hu FB. Exercise type and intensity in relation to coronary heart disease in men. JAMA. 2002;288(16):1994-2000.

63. Mora S, Cook N, Buring JE, Ridker PM, Lee IM. Physical activity and reduced risk of cardiovascular events: potential mediating mechanisms. Circulation. 2007;116(19):2110-2118.

64. Thompson PD, Buchner D, Pina IL, et al. Exercise and physical activity in the prevention and treatment of atherosclerotic cardiovascular disease: a statement from the Council on Clinical Cardiology and the Council on Nutrition, Physical Activity, and Metabolism. Circulation. 2003;107(24):3109-3116.

65. Solecki K, Dupuy AM, Kuster N, et al. Kinetics of high-sensitivity cardiac troponin $\mathrm{T}$ or troponin I compared to creatine kinase in patients with revascularized acute myocardial infarction. Clin Chem Lab Med. 2015;53(5):707-714.

66. Mohlenkamp S, Leineweber K, Lehmann N, et al. Coronary atherosclerosis burden, but not transient troponin elevation, predicts long-term outcome in recreational marathon runners. Basic Res Cardiol. 2014;109(1):391. 
67. Nie J, Close G, George KP, Tong TK, Shi Q. Temporal association of elevations in serum cardiac troponin T and myocardial oxidative stress after prolonged exercise in rats. Eur J Appl Physiol. 2010;110(6):12991303.

68. Howatson G, McHugh MP, Hill JA, et al. Influence of tart cherry juice on indices of recovery following marathon running. Scand J Med Sci Sports. 2010;20(6):843-852.

69. Kharbanda RK, Mortensen UM, White PA, et al. Transient limb ischemia induces remote ischemic preconditioning in vivo. Circulation. 2002;106(23):2881-2883.

70. Kharbanda RK, Nielsen TT, Redington AN. Translation of remote ischaemic preconditioning into clinical practice. Lancet. 2009;374(9700):1557-1565.

71. Sloth AD, Schmidt MR, Munk K, et al. Improved long-term clinical outcomes in patients with ST-elevation myocardial infarction undergoing remote ischaemic conditioning as an adjunct to primary percutaneous coronary intervention. Eur Heart J. 2014;35(3):168-175.

72. Thielmann $M$, Kottenberg $E$, Kleinbongard $P$, et al. Cardioprotective and prognostic effects of remote ischaemic preconditioning in patients undergoing coronary artery bypass surgery: a single-centre randomised, double-blind, controlled trial. Lancet. 2013;382(9892):597-604.

73. Katus HA, Remppis A, Scheffold T, Diederich KW, Kuebler W. Intracellular compartmentation of cardiac troponin $T$ and its release kinetics in patients with reperfused and nonreperfused myocardial infarction. Am J Cardiol. 1991;67(16):1360-1367.

74. Remppis A, Scheffold T, Greten J, et al. Intracellular compartmentation of troponin T: release kinetics after global ischemia and calcium paradox in the isolated perfused rat heart. J Mol Cell Cardiol. 1995;27(2):793-803.

75. Voss EM, Sharkey SW, Gernert AE, et al. Human and canine cardiac troponin T and creatine kinase-MB distribution in normal and diseased myocardium. Infarct sizing using serum profiles. Arch Pathol Lab Med. 1995;119(9):799-806.

76. White HD. Pathobiology of troponin elevations: do elevations occur with myocardial ischemia as well as necrosis? J Am Coll Cardiol. 2011;57(24):2406-2408.

77. Starnberg K, Jeppsson A, Lindahl B, Hammarsten O. Revision of the troponin T release mechanism from damaged human myocardium. Clin Chem. 2014;60(8):1098-1104.

78. Scharhag J, Schneider G, Urhausen A, Rochette V, Kramann B, Kindermann W. Athlete's heart: right and left ventricular mass and function in male endurance athletes and untrained individuals determined by magnetic resonance imaging. J Am Coll Cardiol. 2002;40(10):1856-1863.

79. Utomi V, Oxborough D, Whyte GP, et al. Systematic review and meta-analysis of training mode, imaging modality and body size influences on the morphology and function of the male athlete's heart. Heart. 2013;99(23):1727-1733.

80. Ellison GM, Waring CD, Vicinanza C, Torella D. Physiological cardiac remodelling in response to endurance exercise training: cellular and molecular mechanisms. Heart. 2012;98(1):5-10.

81. Mehta R, Gaze D, Mohan S, et al. Post-exercise cardiac troponin release is related to exercise training history. Int J Sports Med. 2012;33(5):333-337.

82. Mingels A, Jacobs L, Michielsen E, Swaanenburg J, Wodzig W, van Dieijen-Visser M. Reference population and marathon runner sera assessed by highly sensitive cardiac troponin $T$ and commercial cardiac troponin T and I assays. Clin Chem. 2009;55(1):101-108.

83. Legaz-Arrese A, Lopez-Laval I, George K, et al. Impact of an endurance training program on exerciseinduced cardiac biomarker release. Am J Physiol Heart Circ Physiol. 2015;308(8):H913-920.

84. Lee DC, Pate RR, Lavie CJ, Sui X, Church TS, Blair SN. Leisure-time running reduces all-cause and cardiovascular mortality risk. J Am Coll Cardiol. 2014;64(5):472-481.

85. Wen CP, Wai JP, Tsai MK, et al. Minimum amount of physical activity for reduced mortality and extended life expectancy: a prospective cohort study. Lancet. 2011;378(9798):1244-1253.

86. Sharma S, Zaidi A. Exercise-induced arrhythmogenic right ventricular cardiomyopathy: fact or fallacy? Eur Heart J. 2012;33(8):938-940.

87. Sharma S, Merghani A, Mont L. Exercise and the heart: the good, the bad, and the ugly. Eur Heart J. 2015;36(23):1445-1453.

88. Heidbuchel H, Hoogsteen J, Fagard R, et al. High prevalence of right ventricular involvement in endurance athletes with ventricular arrhythmias. Role of an electrophysiologic study in risk stratification. Eur Heart J. 2003;24(16):1473-1480. 
89. Baldesberger S, Bauersfeld U, Candinas $\mathrm{R}$, et al. Sinus node disease and arrhythmias in the long-term follow-up of former professional cyclists. Eur Heart J. 2008;29(1):71-78.

90. Abdulla J, Nielsen JR. Is the risk of atrial fibrillation higher in athletes than in the general population? A systematic review and meta-analysis. Europace. 2009;11(9):1156-1159.

91. Breuckmann F, Mohlenkamp S, Nassenstein K, et al. Myocardial late gadolinium enhancement: prevalence, pattern, and prognostic relevance in marathon runners. Radiology. 2009;251(1):50-57.

92. Benito B, Gay-Jordi G, Serrano-Mollar A, et al. Cardiac arrhythmogenic remodeling in a rat model of long-term intensive exercise training. Circulation. 2011;123(1):13-22.

93. La Gerche A, Burns AT, Mooney DJ, et al. Exercise-induced right ventricular dysfunction and structural remodelling in endurance athletes. Eur Heart J. 2012;33(8):998-1006.

94. La Gerche A, Claessen G, Dymarkowski S, et al. Exercise-induced right ventricular dysfunction is associated with ventricular arrhythmias in endurance athletes. Eur Heart J. 2015.

95. Elliott $A D$, La Gerche $A$. The right ventricle following prolonged endurance exercise: are we overlooking the more important side of the heart? A meta-analysis. Br J Sports Med. 2015;49(11):724-729.

96. Neilan TG, Januzzi JL, Lee-Lewandrowski E, et al. Myocardial injury and ventricular dysfunction related to training levels among nonelite participants in the Boston marathon. Circulation. 2006;114(22):23252333.

97. La Gerche A, Claessen G. Is exercise good for the right ventricle? Concepts for health and disease. Can J Cardiol. 2015;31(4):502-508. 



\section{Summary \\ Samenvatting}




\section{Summary}

The diagnosis of acute myocardial infarction (AMI) relies strongly on the release of cardiac troponins (cTn) (either cardiac troponin I [cTnl] or T [cTnT]) into the bloodstream, which are the preferred biomarkers of myocardial injury. Recent improvement in the analytical performance of the troponin assays resulted in more accurate and sensitive detection of circulating cTn. These high-sensitivity (hs) assays are therefore able to detect cTn in almost every individual of the general population, enabling a precise determination of the $99^{\text {th }}$ percentile cut-off value for the diagnosis of AMI. The clinical introduction of the hs-cTn assays resulted in improved diagnostic sensitivity for $\mathrm{AMI}$ and therefore expedited an early initiation of evidence-based treatment and management. However, this improved sensitivity for AMI has come at a cost of specificity. Elevations of cTn exceeding the diagnostic cut-off for AMI are now detected in patients with acute or chronic cardiomyocyte injury other than AMI, and these elevations are invariably associated with adverse outcomes. Therefore, the universal definition of AMI relies on the detection of a rise and/or fall of cTn above the $99^{\text {th }}$ percentile value of a healthy reference population in conjunction with typical clinical findings of ischemia. There is no consensus about the specific magnitude of change required to diagnose $\mathrm{AMI}$, although a minimum of a $20 \%$ change has been suggested as a rule of thumb. In addition, the accurate detection of hs-cTn in almost every healthy individual of the general population has expanded the possible use of cTn from diagnosis to prognosis. The single exception where it is unclear whether cTn are linked to adverse outcomes is the release of cTn associated with an acute bout of endurance-type exercise. The aim of this thesis was to increase our understanding on how to interpret hs-cTnT and hs-cTnl concentrations in settings of serial testing (chapters 2 and 3), prognostic medicine (chapter 4) and endurance-type exercise (chapters 5, 6 and 7).

Chapter 1 provides a general introduction on cTn, hs assays and the use of hs-cTn in the diagnosis of AMI. Moreover, it discusses the role of serial cTn testing, and the use of biological variation studies in healthy individuals. In addition, the prognostic value of cTn in acute and chronic settings of myocardial injury is described. Furthermore, this chapter provides an overview regarding exercise-induced cTn release, potential contributing mechanisms and strategies to study cTn release in response to exercise.

While hs-cTnl and hs-cTnT are not specific for any particular mechanism of myocardial injury, the critical tool for the diagnosis of AMI is the detection of a 'rise and/or fall' in serially obtained results of hs-cTn. A critical assumption when interpreting kinetic changes is that serial measurements of hs-cTn - in clinically stable conditions - display true random variation around an individual's homeostatic set point. Chapter 2 challenges this critical assumption. Specifically, in a biological variation study with 23 male sub- 
jects with type 2 diabetes, this chapter shows that circulating hs-cTnT exhibits a gradual decrease during the day. The presence of a diurnal rhythm was confirmed by hourly sampling 7 male subjects with type 2 diabetes over $25 \mathrm{~h}$. This diurnal variation is characterized by gradually decreasing values throughout daytime, rising concentrations during nighttime, to peak concentrations in the morning hours. In addition, this chapter demonstrates that the violation of the assumption of random variation has direct consequences for the calculation of the reference change value (RCV), a statistically derived threshold-value based on biological and analytical variation, that distinguishes 'true changes' from 'noise'. Due to the diurnal rhythm of hs-cTnT, the calculated RCV depends strongly on the timeframe chosen. In a period of 'high level changes' (8:30 AM $12: 30 \mathrm{PM})$ the RCV is more than twice the RCV in a period of 'low level changes' $(4: 30$ PM - 8:30 PM).

Chapter 3 validates the diurnal rhythm of hs-cTnT as a universal phenomenon in subjects without acute cardiovascular disease, by hourly sampling of 17 additional men and women over $25 \mathrm{~h}$ with normal glucose homeostasis. Since the diurnal fluctuation of hs-cTnT in some individuals exceeded the suggested delta criteria of $20 \%$ for AMI, these physiological fluctuations have the potential to be interpreted as biochemical evidence of myocardial injury. Therefore, this chapter additionally evaluates whether the diurnal rhythmic oscillation has effect on the diagnostic accuracy of hs-cTnT for AMI. In a multicenter diagnostic trial of 2782 unselected patients presenting to the cardiac emergency department with acute chest pain, this chapter shows that characteristics of the diurnal rhythm persist in real-life chest pain patients with an adjudicated diagnosis of noncardiac disease, by significantly higher levels in patients presenting in the early-morning hours compared to patients presenting to the cardiac emergency department in the evening. Nevertheless, the overall effect of the diurnal hs-cTnT rhythm on the diagnostic accuracy for AMI is fortunately rather small. The diagnostic accuracy of hs-cTnT for $\mathrm{AMI}$ at presentation, $1 \mathrm{~h}$ and the combination of absolute changes with presenting concentrations is very high and independent on the timeframe of patient presentation to the cardiac emergency department. Nevertheless, this chapter shows that the timeframe of patient presentation was more pronounced in patients presenting early after the onset of chest pain and reached statistical significance. In patients presenting in the evening, where the physiological rhythm displays minor fluctuations, absolute changes of hs-cTnT within $1 \mathrm{~h}$ have a significant higher diagnostic accuracy compared to patients presenting in the early-morning, where the diurnal hs-cTnT rhythm displays a high level phase. Therefore, the presence of a diurnal rhythm may provide an explanation in isolated cases of patients where the clinical assessment is discordant with biochemical hs-cTnT kinetics. In addition, this chapter shows that hs-cTnl displays random fluctuations during day and night and that the timeframe of patient presentation has no effect on the diagnostic accuracy of hs-cTnl for AMI. 
The accurate detection of cTn in almost every apparently healthy individual from the general population has expanded the possible use of cTn from diagnostic care to prognostic medicine. Chapter 4 quantifies the relationship between basal levels of hscTn and all-cause mortality during follow-up in the general population in a systematic manner. By a meta-regression analysis of nine studies, this chapter demonstrates a trend towards a better prognostic value of hs-cTnT compared to hs-cTnl.

Endurance-type exercise is currently the only stimulus of cTn release where it is unclear whether the post-exercise levels exceeding the diagnostic cut-off for AMI mirror a physiological or pathological underlying process. Several components of the altered physiological environment of the cardiomyocytes during exercise have been suggested as contributing mechanism, such as increased mechanical stress, elevations in reactive oxygen species and an imbalance between oxygen supply and demand. The aim of chapters 5, 6, and 7 was to study the heterogeneity and CTn release within and between individuals and the underlying mechanism of exercise-induced CTn release. Chapter 5 describes hs-cTnT levels measured in 31 male athletes during two identical highly standardized cycling trials. By a very strong correlation of post-exercise levels during both trials, this chapter demonstrates that exercise-induced hs-cTnT is a reproducible phenomenon. Chapter 6 consists of a double-blind randomized controlled trial, where the effect of 4 weeks antioxidant supplementation was investigated on hs-cTnT release induced by a standardized cycling trial. This chapter demonstrates that exercise-induced hs-cTnT concentrations were not affected by antioxidant supplementation. The aim of chapter 7 was to study the effect of remote ischemic preconditioning (RIPC), a cardioprotective strategy that limits ischemia-reperfusion injury, on the kinetics of hs-cTnl and hs-cTnT following a $30 \mathrm{~km}$ running trial in 25 healthy volunteers. The application of four cycles of temporal episodes of unilateral upper arm ischemia (occlusion to $\geq 220 \mathrm{~mm}$ $\mathrm{Hg}$ ) followed by reperfusion (non-occlusion) before the $30 \mathrm{~km}$ run had no effect on the release kinetics of hs-cTnl and hs-cTnT. In addition, this chapter shows by serially sampling post-exercise that hs-cTnT levels peak earlier than hs-cTnl levels, which is different from the release kinetics in AMI, where peak-levels are reached at similar hours postadmission. All together these findings suggest that cTn release following endurancetype exercise may be a physiological process.

Chapter 8 provides a general discussion of the work described in this thesis. This chapter discusses the diurnal rhythm of hs-cTnT in cardiovascular physiology and pathology. Furthermore, the differences between hs-cTnl and hs-cTnT in terms of their diurnal variation are discussed on the basis of the release and clearance rates. Differences between hs-cTnl and hs-cTnT in terms of their risk stratification are additionally described. Moreover, the clinical significance and the mechanisms of exercise-induced cTn release are reviewed. Finally, directions towards future research are provided. 


\section{Samenvatting}

De diagnose acuut myocardinfarct (AMI) is gebaseerd op het vrijkomen van cardiale troponines (cTn) (cardiaal troponine I [cTnl] of T [cTnT]) in de bloedcirculatie. Deze hart specifieke eiwitten zijn de gouden standaard voor het aantonen van myocardschade. Met de introductie van meer accurate en sensitieve cTn testen is het voor het eerst mogelijk om cTn in de circulatie van gezonde personen te detecteren. Hierdoor wordt de diagnostische afkapwaarde voor een AMI, gedefinieerd als de $99^{\mathrm{e}}$ percentiel concentratie van een gezonde controle groep, nauwkeuriger vastgesteld. Door het gebruik van deze hoog-sensitieve (hs) cTn testen in de diagnostiek is de sensitiviteit verbeterd, waardoor patiënten met een AMI sneller gediagnosticeerd en dus ook eerder behandeld kunnen worden. Deze toename in sensitiviteit gaat helaas gepaard met een afname in specificiteit. Met het gebruik van de hs testen worden ook verhoogde hs-cTn concentraties - boven de diagnostische afkapwaarde - gevonden bij patiënten met andere acute of chronische aandoeningen dan een AMI. Om een AMI te diagnosticeren dient er volgens de universele definitie van AMI sprake te zijn van een stijging en/of daling in hs-cTn waarden, waarbij ten minste één van deze serieel gemeten concentraties is gestegen tot boven de $99^{\mathrm{e}}$ percentiel waarde, samen met andere typische klinische tekenen van ischemie. Het exacte concentratieverschil als afkapwaarde voor de diagnose van een AMI wordt in deze definitie niet besproken, hoewel een $20 \%$ verandering wel als vuistregel wordt genoemd in de literatuur. Behalve voor de diagnostiek blijken cTn ook een rol te spelen als risicovoorspeller voor (cardiovasculaire) morbiditeit en mortaliteit. Deze prognostische waarde is van belang voor het gehele spectrum van hs-cTn concentraties gemeten in verschillende populaties, van hele lage waarden gemeten in het bloed van gezonde personen tot de aspecifieke hs-cTn verhogingen gemeten in patiënten met andere aandoeningen dan een AMI. Echter, de prognostische waarde van hs-cTn concentraties gemeten in individuen uit de algemene bevolking is nog niet systematisch onderzocht. Daarnaast is de klinische en prognostische relevantie van hscTn verhogingen gemedieerd door het beoefenen van duursport vooralsnog onbekend. Het doel van dit proefschrift is om kennis over de interpretatie van hs-cTnT en hs-cTnl te vergroten in het geval van serieel meten (hoofdstuk 2 en 3), prognose (hoofdstuk 4) en duursport (hoofdstukken 5, 6 en 7).

Hoofdstuk 1 geeft een algemene introductie over cTn, hs testen en het gebruik van deze testen in de diagnostiek van een AMI. Daarnaast wordt serieel meten van hs-cTn beschreven als manier om de specificiteit voor een AMI te vergroten en in het bijzonder de rol van biologische variatie bij het bepalen van het exacte concentratieverschil als afkapwaarde. Bovendien wordt de prognostische waarde besproken van hs-cTn verhogingen in patiënten met andere acute en chronische aandoeningen dan een AMI. Ten 
slotte geeft dit hoofdstuk een overzicht over de release van cTn tijdens en na het beoefenen van duursport. Tevens worden de mogelijke mechanismen die hieraan ten grondslag liggen besproken, evenals strategieën om inspanning gemedieerde cTn release te onderzoeken.

Verhoogde hs-cTn concentraties zijn niet specifiek voor myocardschade veroorzaakt door acute ischemie. Hierdoor is serieel meten van hs-cTn concentraties van belang voor de diagnostiek van een AMI. Een belangrijke aanname bij de interpretatie van seriële waarden is dat - in individuen met een klinisch stabiele conditie - hs-cTn concentraties willekeurig fluctueren rondom een evenwicht. Deze essentiële aanname wordt bekritiseerd in hoofdstuk 2. Dit hoofdstuk beschrijft door middel van een biologische variatie studie in 23 mannen met diabetes type 2 dat hs-cTnT concentraties een geleidelijke daling vertonen gedurende de dag. Een dag-nacht ritme in hs-cTnT werd vervolgens bevestigd door ieder uur bloed af te nemen bij 7 mannen met diabetes type 2 gedurende een periode van 25 uur. Dit dag-nacht ritme bestaat uit een geleidelijke daling in hs-cTnT concentraties overdag, gevolgd door een stijging tijdens de nacht, tot piek concentraties in de ochtenduren. Deze onwillekeurige variatie beïnvloedt in sterke mate de berekening van het kritisch verschil ('reference change value'), een vastgestelde delta afkapwaarde die op basis van de biologische en analytische variatie 'echte veranderingen' onderscheidt van 'ruis'. In een periode van 'grote fluctuaties' (8:30 12:30 uur) is het kritisch verschil namelijk twee keer zo groot als het kritisch verschil in een periode met 'kleine fluctuaties' (16:30 - 20:30 uur). Door de aanwezigheid van een dag-nacht ritme in hs-cTnT concentraties is het kritisch verschil dus sterk afhankelijk van het gekozen tijdsbestek.

Hoofdstuk 3 valideert het dag-nacht ritme in hs-cTnT concentraties als een universeel fenomeen. Dit hoofdstuk beschrijft het dag-nacht ritme van hs-cTnT in een additionele groep van 17 mannen en vrouwen met een normale glucose huishouding. Deze onwillekeurige fluctuaties zijn van dusdanige orde dat ze de $20 \%$ afkapwaarde overtreffen. Hierdoor kunnen fysiologische fluctuaties ten onrechte geïnterpreteerd worden als biochemisch bewijs van myocardschade. Het effect van een dag-nacht ritme op de diagnostische waarde voor een AMI is onderzocht aan de hand van een multicenter onderzoek van 2782 ongeselecteerde eerste hart hulp patiënten met klachten van pijn op de borst. Dit hoofdstuk laat zien dat patiënten waarbij een cardiale aandoening is uitgesloten ook aspecten vertonen van het dag-nacht ritme. In deze patiëntengroep hebben namelijk individuen die in de vroege ochtenduren arriveren op de eerste hart hulp met klachten van pijn op de borst significant hogere hs-cTnT concentraties vergeleken met individuen die in de avonduren arriveren op de eerste hart hulp. Desondanks is de diagnostische waarde van hs-cTnT erg hoog en onafhankelijk van het tijdsbestek van binnenkomst op de eerste hart hulp, zoals bepaald aan de hand van hs-cTnT concentraties bij binnenkomst, 1 uur later en de absolute veranderingen binnen 1 uur 
gecombineerd met de concentratie bij binnenkomst. Echter, dit hoofdstuk laat zien dat het tijdsbestek van binnenkomst op de eerste hart hulp een uitgesproken effect heeft in patiënten die snel op de eerste hart hulp arriveren na het ontstaan van pijn op de borst klachten. De diagnostische prestatie van absolute veranderingen in hs-cTnT concentraties binnen 1 uur na binnenkomst is namelijk significant beter bij binnenkomst in de avonduren, een tijdsbestek van relatief kleine fysiologische fluctuaties in vergelijking tot binnenkomst in de ochtenduren, een tijdsbestek van relatief grote fysiologische fluctuaties. Hierdoor is het dag-nacht ritme een mogelijke verklaring voor patiënten waarbij de klinische presentatie niet overeenkomt met seriële hs-cTnT concentraties. Daarnaast beschrijft dit hoofdstuk dat hs-cTnl wél random fluctueert gedurende dag en nacht. Bovendien heeft het tijdsbestek van binnenkomst op de eerste hart hulp geen effect op de diagnostische prestatie van hs-cTnl.

Behalve voor de diagnostiek blijken cTn ook een rol te spelen als risicovoorspeller voor (cardiovasculaire) morbiditeit en mortaliteit. Hoofdstuk 4 beschrijft een systematische evaluatie van de prognostische waarde van lage hs-cTn concentraties voor mortaliteit. Dit hoofdstuk is een meta-analyse van 9 studies die de prognostische waarde van hs-cTnT en/of hs-cTnl concentraties onderzochten in gezonde individuen van de algemene bevolking. De meta-analyse beschrijft een trend voor een betere prognostische waarde van hs-cTnT ten opzichte van hs-cTnl.

Vooralsnog is de klinische en prognostische relevantie van duursport gemedieerde cTn release onbekend. In het bijzonder is het nog onduidelijk of de verhoogde hs-cTn concentraties het gevolg zijn van reversibele of irreversibele myocardschade. Hiervoor is het belangrijk om te vast te stellen welk mechanisme ten grondslag ligt aan het vrijkomen van cTn uit de hartspiercellen. Mogelijke mechanismen die in de literatuur genoemd worden zijn verhoogde mechanische belasting van de hartspiercellen, verhoogde reactieve zuurstofradicalen in het milieu van de hartspiercellen en een verstoring in het evenwicht tussen zuurstofaanvoer en -behoefte van de hartspiercellen. Het doel van de hoofdstukken 5, 6 en 7 was het bestuderen van de heterogeniteit in duursport gemedieerde cTn release op individueel en groeps niveau, evenals het onderliggend mechanisme. Hoofdstuk 5 beschrijft hs-cTnT concentraties gemeten in 31 mannelijke atleten tijdens twee identieke gestandaardiseerde fietstesten. Dit hoofdstuk toont aan dat inspanning gemedieerde cTn release een reproduceerbaar fenomeen is door de sterke correlatie tussen de hs-cTnT waarden direct gemeten na het einde van beide inspanningstesten. Hoofdstuk 6 bestaat uit een dubbelblind gerandomiseerde studie waarbij het effect van 4 weken antioxidant suppletie onderzocht werd op hs-cTnT release gemedieerd door een gestandaardiseerde fietstest. Dit hoofdstuk concludeert dat inspanning gemedieerde hs-cTnT release niet beïnvloed wordt door antioxidant suppletie. Het doel van hoofdstuk 7 was om het effect van ischemische preconditionering te bestuderen op de kinetiek van hs-cTnl en hs-cTnT gemedieerd door een duurloop van 
$30 \mathrm{~km}$. Dit hoofdstuk bestaat uit een enkelblind gerandomiseerde studie van 25 gezonde getrainde vrijwilligers waarbij ischemische preconditionering toegepast werd door middel van een bloeddrukband om één van de bovenarmen. Het toepassen van vier kortdurende cycli van ischemie ( 5 minuten occlusie tot minimaal $220 \mathrm{~mm} \mathrm{Hg}$ ) gevolgd door reperfusie ( 5 minuten geen occlusie) voorafgaand aan de duurloop van $30 \mathrm{~km}$ had geen effect op de release kinetiek van hs-cTnl en hs-cTnT. Daarnaast laat dit hoofdstuk zien dat de kinetiek van hs-cTnl en hs-cTnT gemedieerd door duursport verschilt van de kinetiek in het geval van een AMI. Bij de $30 \mathrm{~km}$ duurloop worden piek concentraties van hs-cTnT eerder waargenomen na het beëindigen van de inspanning dan piek concentraties van hs-cTnl. Dit is verschillend van een AMI waar piek concentraties van hs-cTnT en hs-cTnl worden waargenomen op vergelijkbare tijdstippen na binnenkomst op de eerste hart hulp. De resultaten van deze hoofdstukken suggereren dat het mechanisme van inspanning gemedieerde cTn release verschilt van cTn release in het geval van een AMI.

Hoofdstuk 8 bevat een algemene discussie over de in dit proefschrift beschreven resultaten en de mogelijkheden voor toekomstig onderzoek. In dit hoofdstuk word thet dag-nacht ritme besproken in relatie tot de cardiovasculaire fysiologie en pathologie. Verschillen in de dag-nacht variatie tussen hs-cTnl en hs-cTnT worden bediscussieerd aan de hand van het vrijkomen van cTn uit de hartspiercellen en de klaring van cTn uit de circulatie. Daarnaast worden verschillen in de prognostische waarde van hs-cTnl en hs-cTnT besproken. Ten slotte wordt de klinische relevantie en het mechanisme van inspanning gemedieerde cTn release besproken. 


Valorization 


\section{Introduction}

The aim of this chapter is to address the possible opportunities for knowledge valorization based on the described research findings in this thesis. Knowledge valorization, knowledge transfer or commercialization are similar terms that all refer to the "process of creating value from knowledge, by making knowledge suitable and/or available for social and economic use and by making knowledge suitable for translation into competitive products, services, processes and new commercial activities".,

\section{Relevance}

Acute myocardial infarction (AMI) and (unstable) angina, components of the clinical definition '(acute) coronary syndrome', are major causes of death and disability worldwide. $^{3}$ In 2011, 9,876 individuals in the Netherlands died of coronary disease, with the majority of deaths $(n=6,467,65 \%)$ resulting from AMI. ${ }^{4}$ In the same year, 115,921 subjects with coronary disease were admitted to a hospital in the Netherlands, which accounted for $3 \%$ of all hospital admissions in the Netherlands in $2011 .{ }^{4}$ This resulted in an economic healthcare burden of $€ 2.1$ billion, which corresponded to $2.3 \%$ of the total amount of healthcare costs in 2011 in the Netherlands. ${ }^{5}$

Patients with acute chest pain or other symptoms suggestive of AMI account for more than $10 \%$ of all emergency department consultations. ${ }^{6}$ As the majority of these patients will be found not to have AMI, the diagnosis (rule-in) and rule-out of AMI seems to be equally important. ${ }^{7}$ In addition, rapid and accurate diagnosis of AMI is of paramount clinical importance: delayed 'rule-in' of AMI increases morbidity and mortality, whereas delayed 'rule-out' causes anxiety in patients and excessive health care costs due to prolonged time spent at the emergency department. ${ }^{8}$ Most patients with AMI have a non-diagnostic ECG and require serial blood sampling and measurement of cardiac troponins (cTn) as part of the clinical workup. ${ }^{3}$ The clinical presentation of the patient, in conjunction with a typical 'rise or fall' of cTn concentrations between serial measurements, establishes the diagnosis. ${ }^{3}$ In 2013, cTn were measured in 3,585 blood samples collected from patients presenting at the cardiac emergency department of Maastricht University Medical Center.

The analytical performance of the troponin assays has improved enormously over the last 5 years. Patients diagnosed with the 'high-sensitivity' (hs) troponin assays demonstrate a clear survival benefit compared to patients diagnosed with conventional troponin assays, due to an improved diagnostic sensitivity. ${ }^{9}$ However, the diagnosis of AMI with the hs assays is more complex, which arises from the detection of previously 
unnoticed cTn elevations in patients with cardiomyocyte injury other than AMI. ${ }^{10}$ This includes acute heart failure, type 2 diabetes, and even cTn elevations in healthy recreational athletes after an acute bout of endurance-type exercise. This lack in specificity is a challenge for clinicians. Therefore, it is essential to increase our understanding on how to interpret levels of cTn in settings of serial testing and endurance-type exercise.

On the contrary, the improvement in analytical sensitivity has resulted in more applications of cTn. Even when adjusted for traditional cardiovascular risk factors (such as smoking, diabetes, hyperlipidemia and hypertension), cTn concentrations below the clinical cut-off value for AMI measured in the general population are strongly associated with an increased risk of developing cardiovascular events and all-cause or cardiovascular mortality. ${ }^{11}$ As traditional risk factors do not identify everyone who will eventually develop cardiovascular disease, ${ }^{12}$ cTn are promising candidates to refine risk stratification models for the primary prevention of cardiovascular disease.

\section{Target groups}

Interpretation of troponin levels are mainly performed by clinicians (e.g. cardiologists, general practitioners) and laboratory specialists. In addition, the results described in this thesis could be of interest to diagnostic companies (e.g. Roche Diagnostics, Abbott Diagnostics, Beckman Coulter) that are involved with the development, optimization, implementation or application of a specific troponin assay. Research described in this thesis also has the potential to be of interest for (recreational) athletes.

\section{Activities}

The results described in this thesis can be translated into several activities or tools. These activities are clustered according to the three mechanisms of knowledge transfer; through results or commercialization, people and via cooperation (figure 1).

This thesis describes the presence of a diurnal rhythm of cTn. As this physiological fall-and-rise during day and night may provide an explanation in isolated cases of patients where the clinical assessment is discordant with biochemical results, awareness of the presence of a physiological rhythm is important among individuals that interpret concentrations of cTn. This can be achieved via interaction with clinicians and laboratory specialists (e.g. presentations, dialogues, conferences) or via lectures for medicine students of Maastricht University Medical Center. This level of knowledge utilization has already been started through publication of results in the Journal of the American College of Cardiology, a high impact cardiology journal, and through oral presentations at 
national and international conferences (IFCC, Istanbul, 2014). Additional novel insights arising from future studies will further enhance awareness through publications and presentations. The second step of knowledge utilization is to connect with key-opinion leaders that comprise the writing group on behalf of the joint ESC / ACCF / AHA / WHF Task Force for the Universal Definition of Myocardial Infarction. ${ }^{3}$ This level of knowledge transfer has been initiated by the collaboration with the research group of Prof. dr. Mueller (University Hospital Basel, Switzerland), a senior scientist behind major breakthroughs related to the optimal diagnostic algorithm of cTn for the diagnosis of AMI. The final step is the actual integration of the diurnal rhythm into guidelines such as the expert consensus document 'Universal definition of myocardial infarction'. ${ }^{3}$

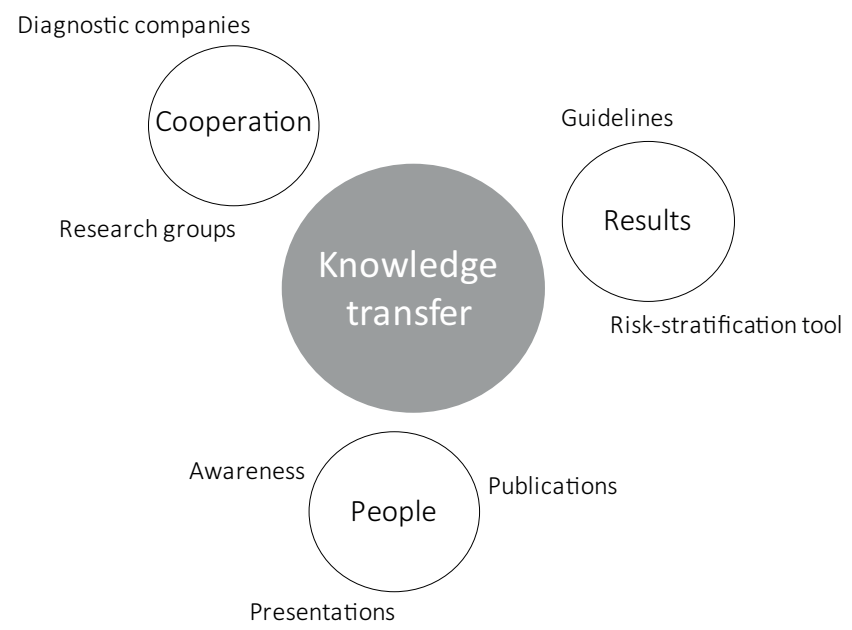

Figure 1. Potential knowledge transfer activities based on the research findings described in this thesis.

Results of this thesis support the concept that cTn can be implemented into riskstratification scores to identify individuals in the general population at risk for cardiovascular diseases. The national guideline for general practitioners recommends to determine the risk and need for prevention consultation for patients according to a medical risk questionnaire, without the measurement of biomarkers. ${ }^{13}$ To initiate the first step of knowledge utilization (awareness), future studies should aim to determine the additional value of cTn concentrations in this risk calculation tool used by general practitioners.

The research findings concerning exercise-induced cTn release have the potential to be utilized by (recreational) athletes. The first step would be to organize information meetings and discuss the effects of endurance-type exercise. To initiate this first step of 
valorization, more studies are needed to make a definite statement regarding the underlying process (i.e. physiological or pathological) of exercise-induced cTn release.

Troponin testing involves the measurement of one of two proteins - cardiac troponin $\mathrm{T}(\mathrm{cTnT})$ or I (cTnl) - with distinct biochemical properties, but comparable diagnostic performance. ${ }^{14}$ A recent European survey demonstrated that $50 \%$ of the inquired laboratories use cTnT and 45\% use cTnl concentrations as part of the diagnostic workup for AMI. ${ }^{15}$ This thesis described minor differences between cTnT and cTnl in terms of their diurnal variation and prognostic value. Future studies should aim to compare cTnT and $\mathrm{cTnl}$ measured by hs assays across the broad spectrum of cardiovascular disease. Awareness of these comparisons among the individuals that interpret cTn concentrations have the potential to result in a pathophysiological preference of one troponin biomarker over another and could influence the troponin test offered by the clinical laboratory.

cTn are already incorporated into clinical practice. Therefore, research focusing on the biomarker has the potential for cooperation with diagnostic companies regarding for example the implementation and evaluation of troponin assays. The first level in this knowledge transfer activity has already been initiated, as the Central Diagnostic Laboratory of Maastricht has excellent collaborations with Roche Diagnostics, Abbott Diagnostics and Beckman Coulter.

\section{Conclusion}

Research findings of this thesis can be translated into several knowledge transfer activities involving people, results and cooperation. Although the outcomes of these mechanisms of knowledge utilization are different, the first step, i.e. creating awareness through publications or presentations, is identical among all knowledge utilization activities. 


\section{References}

1. VSNU. Een Raamwerk Valorisatie-indicatoren. www.vsnu.nl/valorisatie2013.

2. Finne H, Day A, Piccaluga A, Spithoven A, Walter P, Wellen D. A Composite Indicator for Knowledge Transfer. 2011.

3. Thygesen K, Alpert JS, Jaffe AS, et al. Third universal definition of myocardial infarction. Eur Heart J. 2012;33(20):2551-2567.

4. Centraal Bureau voor de Statisiek. StatLine. statline.cbs.nl.

5. RIVM. Kosten van Ziekten. www.kostenvanziektentool.volksgezondheidenzorg.info.

6. Nawar EW, Niska RW, Xu J. National Hospital Ambulatory Medical Care Survey: 2005 emergency department summary. Adv Data. 2007(386):1-32.

7. Mueller C. Biomarkers and acute coronary syndromes: an update. Eur Heart J. 2014;35(9):552-556.

8. Wiese M. Reducing short-stay hospital admissions by ruling out non-ST elevation myocardial infarction and estimating coronary artery disease likelihood on an emergency department observation ward. BMJ Qual Improv Report. 2013;2.

9. Reichlin T, Hochholzer W, Bassetti S, et al. Early diagnosis of myocardial infarction with sensitive cardiac troponin assays. N Engl J Med. 2009;361(9):858-867.

10. Giannitsis E, Katus HA. Cardiac troponin level elevations not related to acute coronary syndromes. Nat Rev Cardiol. 2013;10(11):623-634.

11. de Lemos JA, Drazner MH, Omland T, et al. Association of troponin T detected with a highly sensitive assay and cardiac structure and mortality risk in the general population. JAMA. 2010;304(22):25032512.

12. Khot UN, Khot MB, Bajzer CT, et al. Prevalence of conventional risk factors in patients with coronary heart disease. JAMA. 2003;290(7):898-904.

13. NHG. NHG-standaard Het PreventieConsult. www.nhg.org/standaarden.

14. Rubini Gimenez M, Twerenbold R, Reichlin T, et al. Direct comparison of high-sensitivity-cardiac troponin I vs. T for the early diagnosis of acute myocardial infarction. Eur Heart J. 2014;35(34):23032311.

15. Pulkki K, Suvisaari J, Collinson P, et al. A pilot survey of the use and implementation of cardiac markers in acute coronary syndrome and heart failure across Europe. The CARdiac MArker Guideline Uptake in Europe (CARMAGUE) study. Clin Chem Lab Med. 2009;47(2):227-234. 




\section{Dankwoord \\ Curriculum vitae List of publications}




\section{Dankwoord}

Het schrijven van een proefschrift is eigenlijk maar een klein deel van een promotietraject. En juist alles wat erbij komt kijken maakt promoveren tot een leerzame, verrijkende en onvergetelijke ervaring: het inplannen en organiseren van testdagen, het labelen van (bloed)buizen, 30 uur lang wakker zijn tijdens de eerste dagnacht testen, kilometers meefietsen tijdens de hardloopstudie, cardiaal troponine metingen verrichten in Maastricht, Sittard en Apeldoorn, statistiek, artikels schrijven, presentaties geven, congresbezoeken en niet te vergeten talloze koffiepauzes (al ben ik nog steeds niet echt fan van koffie). Ik ben blij dat ik dit hoofdstuk mag sluiten en daarvoor wil ik de volgende mensen bedanken.

Allereerst mijn promotieteam: Prof. dr. Marja van Dieijen-Visser, Prof. dr. Luc van Loon en Dr. Steven Meex. Beste Marja, ondanks je drukke werkzaamheden ben je altijd heel betrokken geweest bij mijn onderzoek. Naast het feit dat je iedere keer tijd vrijmaakte voor de cardio meetings, stond ik er telkens van te kijken hoe snel jij een manuscript nagekeken had. Daarnaast was ik erg vereerd met je aanwezigheid bij het ontvangen van de NVKC wetenschapsprijs. Beste Luc, bedankt voor al je inbreng. Je inspireerde en dacht altijd na over nieuwe initiatieven, zoals die ene keer dat je suggereerde om cardiaal troponine levels te meten in type 2 diabeten in sedentaire condities. Zonder jouw standvastigheid was het dagnacht ritme, een belangrijk onderdeel van mijn proefschrift, onbesproken gebleven. Bedankt voor jouw visie op het troponine onderzoek en de faciliteiten die jouw onderzoeksgroep mij geboden heeft. Beste Steven, vanaf de eerste dag was jij er voor de dagelijkse begeleiding en het uitzetten van de grote lijnen van mijn promotietraject. Bedankt dat ik altijd bij je langs kon lopen met vragen. Jouw term 'storyboarden' zal ik niet snel vergeten. Waar ik in het begin nog wel eens schrok van je opmerkingen op een eerste versie van een manuscript, keek ik naarmate het promotietraject vorderde steeds meer uit naar je feedback. Bedankt voor de mogelijkheid om mezelf te ontwikkelen.

De leden van de beoordelingscommissie: Prof. dr. Stehouwer, Prof. dr. Apple, Prof. dr. Glatz, Prof. dr. Van Solinge en Prof. dr. De Windt, hartelijk dank voor het beoordelen van dit proefschrift. Dear Prof. dr. Apple, it is a great honor that you, as an internationally recognized expert in the field of cardiac biomarkers, are a member of my assessment committee.

Dankjewel aan alle collega's van het Centraal Diagnostisch Laboratorium (CDL)! Prof. dr. Otto Bekers, beste Otto, bedankt voor je gedetailleerde blik op mijn manuscripten. 
Beste stafleden, bedankt voor jullie interesse in mijn onderzoek en jullie kritische blik tijdens presentaties en overleggen.

Eline, ondanks het feit dat jij al een jaar bezig was toen ik me bij het troponine clubje voegde, waren we al gauw een team. Bedankt voor de tripjes naar Milaan, Istanbul en Barcelona, de jalalalalalalalalala-momentjes, de koffie'tjes, Oreo's en de snelle Jelle's. Ik vind het fijn dat ik dit met jou mocht delen. Sander, bedankt voor al je raad en daad als enige man en ingenieur in ons clubje. Noreen, als student-assistent en vervolgens collega: dankjewel voor je hulp. Tijdens de gezamenlijke testdagen/nachten hebben we een hoop beleefd, gegeten en vastgelegd op foto. Dorien en Judith, allebei super lieve collega's, bedankt voor jullie oprechte interesse in mij en mijn onderzoek. Anne-Hilde, Frederique, Linda en Minka, bedankt voor jullie gezelligheid, jullie zijn een fijne aanvulling op het troponine clubje. Ik wens jullie allemaal heel veel succes toe met jullie onderzoek en ik kijk uit naar jullie proefschriften!

Alma, bedankt voor het delen van je uitgebreide troponine kennis tijdens presentaties en discussies. Vincent, naast het feit dat je voor mij onmisbaar bent geweest in het lab, heb je je tijdens mijn studies ook ontplooid tot fietser en maaltijdverzorger. Ik wil je dus ontzettend bedanken voor alle hulp (vaak buiten kantooruren!) die je mij geboden hebt. Petal, bedankt voor jouw streng, doch rechtvaardig toezicht ;-) op niet alleen ons labje, maar ook onze werkzaamheden op kantoor. Bedankt voor de leuke tijd! Ook dankjewel aan student-assistent Daniëlle Snijders, voor alle vrije uurtjes die je hebt meegedraaid met de hardloopstudie.

Richard en Maurice, het komisch duo, bedankt voor alle hulp met betrekking tot bestellingen of het vinden van bepaalde buisjes of dopjes. Ook de dames en heer van CDL cluster 1 en 2: Frank, Irene, Karin, Kitty en Nina, bedankt voor alle gezelligheid in de wandelgangen maar ook tijdens de (Kerst)feestjes of de andere uitjes. Joyce, Manuela, Meriam en Paula, bedankt voor alle gezellige lunchpauzes. Mijn voorgangers en excollega's; Bianca, Henrike, Lenneke, Leo en Marianne verdienen ook nog een eervolle vermelding in dit dankwoord voor hun voorbeeldige indruk en solide basis die ze aan het lab gegeven hebben.

Bedankt M3 research unit dat ik regelmatig op jullie mocht terugvallen! Jan-Willem van Dijk, dank voor al je hulp. Niet alleen met het opzetten en plannen van de eerste dagnacht testen, maar ook met de goede adviezen die je me in de (rustige) nachtelijke uurtjes gaf. Het was een eer om met je samen te mogen werken. Ook dank aan Bart Groen, Imre Kouw en Peter Res voor jullie uitgebreide kennis en ervaring in het uitvoe- 
ren van klinisch onderzoek. Fijn dat ik altijd kon binnenlopen. Daarnaast wil ik Joan Senden en Antoine Zorenc bedanken voor alle infusen die ze geprikt hebben.

Alle deelnemers aan het onderzoek, ietwat oneerbieding 'proefpersonen' genoemd in ons jargon, zonder jullie was dit onderzoek onmogelijk geweest. Bedankt!

Vruchtbare samenwerkingen zijn van essentieel belang in wetenschappelijk onderzoek. Bedankt aan alle coauteurs voor hun bijdrage aan dit proefschrift.

Afdeling Cardiologie van het MUMC+, bedankt voor jullie inspanningen met betrekking tot de hardloopstudie.

Dear Prof. dr. Mueller and Dr. Wildi, thank you very much for our fruitful collaboration regarding the consequences of the diurnal cardiac troponin rhythm for clinical practice. I hope that our manuscript will be accepted soon.

Zo heb ik troponine gemeten in verschillende ziekenhuizen. Graag wil ik Dr. Jeroen van Suijlen, Dr. Laurens-Jan Jellema en Dr. Marijke Niens bedanken voor hun hulp en de mogelijkheid om hs-cTnl metingen uit te voeren in het Gelre ziekenhuis te Apeldoorn. Daarnaast wil ik Dr. Joop ten Kate bedanken voor de mogelijkheid om ook in SittardGeleen troponine I te bepalen.

Dr. Maarten Raijmakers, bedankt voor het opsturen van de troponine restreagentia.

In mijn vrije tijd ben ik regelmatig omringd door familie en vrienden. Gelukkig ook maar, want een hele dag in je eentje thuis zitten is een dag niet geleefd. Alle mensen die betrokken zijn geweest in dit Lieke-entertainment wil ik dan nu ook hartelijk bedanken.

Wat ik erg koester is het contact met mijn mede-Rolducienne, MLWers en MMDer. Ook al zien we elkaar niet meer zo vaak als toen, het is leuk om te zien hoe een ieder zijn eigen leven invult. Lieve Jonna, ik vind het leuk dat we na Rolduc nog regelmatig contact hebben en wat mij betreft houden we onze eet-traditie nog lang in stand. Ondanks onze verschillende achtergrond denken we op veel gebieden hetzelfde, bedankt voor alle fijne gesprekken die daardoor ontstaan. Ook een welgemeend dankjewel aan de MLWvriendinnetjes; Monique, Nadine en Nicki. Bedankt voor jullie oprechte interesse, steun en afleiding door alle leuke uitjes. Ik hoop dat we snel nog een keer compleet zijn met $z^{\prime} n$ viertjes. Lieve Rike, ik vind het erg leuk dat we ook na Nijmegen nog regelmatig contact hebben. Bedankt voor je luisterend oor tijdens de gezellige shop- en high teauitjes. 
En natuurlijk wil ik ook graag de mensen bedanken met wie ik spelletjes win: Martijn, Caroline, Joeri, Daniëlle, Robbert, Inge, Olga en Cedric. Pubquiz crews Jen \& Berry's, $E=M C$ Hammer en Burning Wookiees, bedankt voor de vele top drie noteringen bij de quiz, dat er nog meer mogen volgen! Room escape crew SwashBuckling Buckaneers: wauw, wat hebben wij al leuke dingen beleefd. Ik hoop dat we nog in een hoop uithoeken van Nederland terecht zullen komen!

De beste beslissing aan het begin van mijn promotietraject was mijn lidmaatschap bij il Fiore. Sinds mijn allereerste werkdag fietste ik regelmatig vanuit werk naar huis met een kleine omweg;-). Een speciaal woord van dank aan de bikkels: Cedric, Efke, Jacques, Janneke, Jeroen, Myrurgia, Pascal, Patrick en Stefan. 'HEUY' Ik ben ontzettend blij dat ik echt mezelf kan zijn met jullie in de lessen ' $\mathrm{OOH}-\mathrm{OOH}^{\prime}$. In de afgelopen vier jaar hebben we van alles uitgespookt: badmutsen, Harlem Shake, Halloween-, Hawaï- en Kerstoutfits en ook buiten il Fiore is het altijd hartstikke gezellig. Daarnaast hebben jullie, zeker aan het einde van mijn promotie, altijd geïnformeerd naar mijn onderzoek, waarvoor dank!

Ook een welgemeend dankjewel aan mijn schoonfamilie: Claudia, Caroline, Joeri, Danielle en Robbert. Het motto voor al onze gezellige momenten was het creëren van een "leeg sjäosje". Bedankt voor de keren dat we het uit de hand lieten lopen tijdens de 'boozapaloozas', 'de drakenpoort', de opstanden tijdens de 'grote Dalmuti' (hihi ;-) ), en niet te vergeten onze jaarlijkse filmmarathon rond de feestdagen. Lieve Caroline, ik ben ervan overtuigd dat jij een waardevolle ondersteuning zult zijn tijdens mijn verdediging. Ontzettend bedankt dat je mijn paranimf wilt zijn. Claudia, bedankt voor het faciliteren van alle bovenstaande activiteiten. Lief dat je altijd zo geïnteresseerd in en betrokken bent bij Martijn en mij.

Ook wil ik Bert bedanken voor zijn interesse in mijn onderzoek.

Lieve Inge, Rianne, Rob en Silke, bedankt voor al jullie steun! Inge, bedankt voor je goede adviezen tijdens mijn promotietraject. Heel fijn dat ik de stijl van jouw proefschrift mocht gebruiken als cover voor mijn eigen proefschrift. Samen met het boekje van pap staan ze alle drie zeker weten mooi naast elkaar in de boekenkast. Ik vond het ook heel speciaal om samen met jou de BKO-cursus te doen, dat had je toch maar even goed geregeld;-). Rianne, ik vind het ontzettend tof dat jij als grote zus mijn paranimf bent! Zo is de cirkel tussen Inge, jou en mij rond. Bedankt voor al je interesse in wat ik doe en je waardering over wie ik ben en wat ik kan. Rob, je bent een fijne aanvulling in onze familie en op Rianne. Silke, ook al ken ik je nog maar net, ik geniet ervan om bij je te zijn. 
Lieve papa en mama, bedankt dat jullie me altijd gesteund, gestimuleerd en gemotiveerd hebben om het beste uit mezelf te halen. Dat ik zo ver gekomen ben, is mede te danken aan de basis die jullie mij gegeven hebben. Bedankt dat jullie letterlijk en figuurlijk altijd voor ons drieën klaar staan, ongeacht in goede of in minder goede tijden. Pap, naarmate het eind van mijn promotietijd dichterbij kwam, besefte ik me pas wat voor een held jij bent met jouw eigen promotie 21 jaar geleden. Ik geniet ontzettend van onze gezamenlijke passie voor Roda JC Kerkrade en ik hoop dat we die Koempel-genen nog lang in de familie zullen houden. DANKE BITTE! Mam, bedankt dat je altijd zo positief denkt en dit met me deelt door middel van kaartjes, berichtjes, plantjes, bloemetjes, feestpakketten, noem maar op. Ook al is mijn kamertje al lang mijn kamertje niet meer, ik weet dat ik bij Hotel Mama altijd terecht kan. Dankjewel! Ook een woord van dank aan klus- en verhuisbedrijf Klinkenberg, van Kerkrade naar Maastricht, Nijmegen, Londen en weer terug naar Maastricht, jullie laten me nooit in de steek. Heb ik nog credits over trouwens?

Lieve Martijn, de laatste woorden van dit dankwoord zijn vanzelfsprekend voor jou. Bedankt voor al je onbeschrijfelijke steun, liefde en vertrouwen. Ook niet te vergeten je luisterend oor, je advies en de moeite die je telkens weer deed om mij te laten afschakelen als ik het (even) niet meer zag zitten. Ik word er steeds beter in, toch ;-)? Ook dankjewel voor alle ontelbare keren dat we 's avonds iets later gingen eten omdat ik moest en zou gaan sporten, dat je een belangrijke tekst voor mij nalas, en dat je uit bittere noodzaak een dagje meefietste bij de hardloopstudie (3 uur, 13 minuten en 36 seconden ;-) ). Herinner je je nog de spreuk die we tegenkwamen in het restaurant in Húsavík tijdens onze IJslandreis? Nu hopelijk weer meer tijd voor pret! Dikke kus! 


\section{Curriculum vitae}

Lieke Klinkenberg was born on March 9, 1987 in Brunssum, the Netherlands. She graduated in 2005 from secondary school (VWO) at College Rolduc in Kerkrade. In September 2005, Lieke started studying Molecular Life Sciences at Maastricht University. After obtaining her Bachelor's degree cum laude in 2008, she was admitted to the Research Master's programme Fundamental Neuroscience of Maastricht University in September 2008. In September 2009, Lieke switched to the Research Master's programme Molecular Mechanisms of Disease at Radboud University Nijmegen. She performed her first Master internship at the department of Tumorimmunology of Radboud University Nijmegen, under the supervision of Prof. Jolanda de Vries where she worked on dendritic cell subsets. For her Master's thesis she visited the department of Virology of Imperial College London, UK, where she studied the replication of murine norovirus under the supervision of Prof. Ian Goodfellow. In September 2011, Lieke graduated with the distinction summa cum laude. Subsequently, in October 2011, she started as a PhDstudent at the department of Clinical Chemistry / Central Diagnostic Laboratory of Maastricht University Medical Center under the supervision of Prof. Marja van DieijenVisser, Prof. Luc van Loon and Dr Steven Meex. In 2014, she was invited at IFCC Worldlab in Istanbul to present her data regarding the diurnal cardiac troponin $T$ rhythm, which was published in 2014 in the Journal of the American College of Cardiology. For the same paper, she received the Clinical Chemistry Science Award from the Netherlands Society of Clinical Chemistry and Laboratory Medicine (NVKC) for the highest rated publication of 2014. 


\section{List of publications}

In this thesis

Lieke Klinkenberg*, Jan-Willem van Dijk*, Frans Tan, Luc van Loon, Marja van DieijenVisser, Steven Meex. Circulating cardiac troponin T exhibits a diurnal rhythm. Journal of the American College of Cardiology. 2014. 63(17):1788-1795 (impact factor 15.3).

Lieke Klinkenberg*, Peter Res*, Guido Haenen, Aalt Bast, Luc van Loon, Marja van Dieijen-Visser, Steven Meex. Effect of antioxidant supplementation on exercise-induced cardiac troponin release in cyclists: a randomized trial. PLoS One. 2013. 8(11):e79280 (impact factor 3.5).

Lieke Klinkenberg*, Peter Res*, Luc van Loon, Marja van Dieijen-Visser, Steven Meex. Strong link between basal and exercise-induced cardiac troponin T levels: do both reflect risk? International Journal of Cardiology. 2012. 158(1):129-131 (impact factor 5.5).

Lieke Klinkenberg*, Karin Wildi*, Noreen van der Linden, Imre Kouw, Marijke Niens, Raphael Twerenbold, Maria Rubini Gimenez, Christian Puelacher, Jean Daniel Neuhaus, Petra Hillinger, Thomas Nestelberger, Jasper Boeddinghaus, Karin Grimm, Jeroen van Suijlen, Frans Tan, Joop ten Kate, Otto Bekers, Luc van Loon, Marja van Dieijen-Visser, Christian Mueller, Steven Meex. A diurnal rhythm of cardiac troponin: consequences for the diagnosis of acute myocardial infarction. Submitted.

Lieke Klinkenberg, Peter Luyten, Noreen van der Linden, Kim Urgel, Daniëlle Snijders, Christian Knackstedt, Robbert Dennert, Bastiaan Kietselaer, Alma Mingels, Eline Cardinaels, Frederique Peeters, Jeroen van Suijlen, Joop ten Kate, Elke Marsch, Thomas Theelen, Judith Sluimer, Kristiaan Wouters, Otto Bekers, Sebastiaan Bekkers, Luc van Loon, Marja van Dieijen-Visser, Steven Meex. Cardiac troponin T and I release following a 30 $\mathrm{km}$ run: comparison of troponin kinetics and effect of remote ischemic preconditioning. Submitted.

Noreen van der Linden, Lieke Klinkenberg, Otto Bekers, Luc van Loon, Marja van DieijenVisser, Maurice Zeegers, Steven Meex. Prognostic value of basal high-sensitive cardiac troponin levels on mortality in the general population: a systematic review and metaanalysis. Submitted.

*Equal contribution 


\section{Additional publications}

Lieke Klinkenberg, Otto Bekers, Steven Meex. Update: Europese NSTEMI richtlijn 2015: 0-1 uurs algoritme voor cardiaal troponine. Nederlands tijdschrift voor Klinische Chemie en Laboratoriumgeneeskunde. 2016. 41:49-53.

Noreen van der Linden, Lieke Klinkenberg, Marika Leenders, Michael Tieland, Lex Verdijk, Marijke Niens, Jeroen van Suijlen, Lisette de Groot, Otto Bekers, Luc van Loon, Marja van Dieijen-Visser, Steven Meex. The effect of exercise training on the course of cardiac troponin $T$ and I levels: three independent training studies. Scientific Reports. Accepted (impact factor 5.6).

Noreen van der Linden, Lieke Klinkenberg, Steven Meex, Erik Beckers, Norbert de Wit, Lenneke Prinzen. Immature platelet fraction measured on the Sysmex XN hemocytometer predicts thrombopoietic recovery after autologous stem cell transplantation. European Journal of Haematology. 2014. 93(2):150-156 (impact factor 2.4).

Gerty Schreibelt, Lieke Klinkenberg, Luis Cruz, Paul Tacken, Jurjen Tel, Marin Kreutz, Gosse Adema, Gordon Brown, Carl Figdor, Jolanda de Vries. The C-type lectin receptor CLEC9A mediates antigen uptake and (cross-)presentation by human blood BDCA3+ myeloid dendritic cells. Blood. 2012. 119(10):2284-2292 (impact factor 9.1).

Noreen van der Linden, Michael Tieland, Lieke Klinkenberg, Lex Verdijk, Lisette de Groot, Luc van Loon, Marja van Dieijen-Visser, Steven Meex. The effect of a six-month resistance-type exercise training program on the course of high sensitive cardiac troponin T levels in (pre)frail elderly. International Journal of Cardiology. 2014. 175(2):374375 (impact factor 6.2).

Marja van Dieijen-Visser, Alma Mingels, Leo Jacobs, Eline Cardinaels, Will Wodzig, Otto Bekers, Lieke Klinkenberg, Steven Meex. Troponin, the best there is! Nederlands tijdschrift voor Klinische Chemie en Laboratoriumgeneeskunde. 2012. 37:15-18. 